\title{
World Congress of Perinatal Medicine ISTANBUL-2019/September 11-14
}

\section{4th World Congress of Perinatal Medicine (WCPM)}

September 11-14, 2019, Istanbul, Turkey

ICEC - Istanbul Lütfi Kırdar Convention and Exhibition Centre www.wcpm2019.or

\section{POSTER PRESENTATIONS}

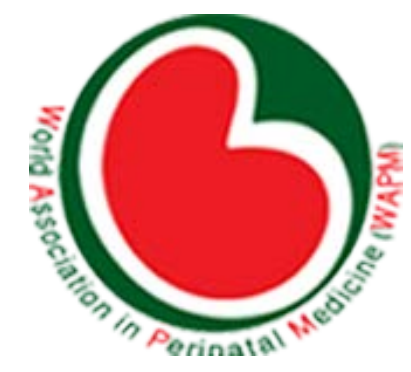




\title{
Obstetrics - Fetal echocardiography and Congenital Heart Diseases
}

\author{
E1060 - PRENATAL ECHOCARDIOGRAPHIC FINDINGS IN FETUSES WITH \\ TRANSPOSITION OF THE GREAT ARTERIES TO PREDICT THE NEED FOR \\ BALLOON ATRIAL SEPTOSTOMY \\ Mizuno Y. ${ }^{1}$, Tsuritani M. ${ }^{1}$, Shionoiri T. ${ }^{1}$, Kyomoto M. ${ }^{1}$, Matsuzaka Y. ${ }^{1}$, Tsukinaga R. ${ }^{1}$, \\ Sawada M. ${ }^{1}$, Konishi T. ${ }^{1}$, Nakanishi A. ${ }^{1}$, Horiuchi C. ${ }^{1}$, Kamiya C. ${ }^{1}$, Iwanaga N. ${ }^{1}$, \\ Kurosaki K. ${ }^{2}$, Yoshimatsu J. ${ }^{1}$ \\ National Cerebral and Cardiovascular Center, Perinatology, Osaka-Japan ${ }^{1}$ \\ National Cerebral and Cardiovascular Center, Pediatric Cardiology, Osaka-Japan ${ }^{2}$
}

\section{Objective}

Prenatal diagnosis of transposition of the great arteries (TGA) has been progressing, and the surgical outcome from the arterial switch operation is usually successful. However, some neonates, especially who have intact ventricular septum or small ventricular septal defect (VSD), show severe hypoxemia soon after birth and even die in preoperative period. In such cases, immediate balloon atrial septostomy (BAS) is needed in order to improve mixing of the pulmonary and systemic circulation. We aimed to identify the prenatal echocardiographic features that may predict the need for urgent BAS.

\section{Methods}

We reviewed the echocardiograms of the fetuses prenatally diagnosed as TGA and managed at our institution between January 2012 and December 2018, retrospectively. We assessed their ductus arteriosus and foramen ovale on the basis of the past several studies. We measured the diameter of the ductus arteriosus and reviewed the Doppler flow pattern. Regarding the analysis of the atrial septum, we measured the maximal angle of flap of the foramen ovale and calculated the ratio of the maximal bulging of the foramen ovale towards the left atrial wall to the left atrial width, as well as the ratio of the foramen ovale length to the atrial septal length. We also focused on the shape of the foramen ovale.

\section{Results}

Among all 22 cases, 12 were included as TGA with intact ventricular septum or small VSD. 6 of them received the urgent BAS within 3 days after birth. The median gestational age at echocardiographic exam was 32 weeks in both fetuses who needed BAS and who didn't. The gestational age at birth and birth weight were not significantly different between the fetuses as well. There was no significant difference in the arterial duct $\mathrm{z}$ score, and no one had reverse ductal flow. The flap angle of the foramen ovale and ratio of length of foramen ovale to atrial septum were significantly small in patients who received urgent BAS in comparison with those who didn't. Hypermobility of the foramen ovale, which is defined as oscillation of its flap valve between the left and right atrium, was seen in 3 (50\%) cases.

Conclusion: The angle of flap of the FO, and FO to atrial septum length ratio which were obtained by fetal echo cardiogram can be helpful for predicting the need for urgent BAS after birth. 


\section{E1061 - THE PRENATAL DIAGNOSIS OF LONG QT SYNDROME}

Tsukinaga $R .{ }^{1}$, Kamiya C. ${ }^{1}$, Kyomoto M. ${ }^{1}$, Mizuno Y. ${ }^{1}$, Matsuzaka Y. ${ }^{1}$, Sawada M. ${ }^{1}$, Shionoiri T. ${ }^{1}$, Konishi T. ${ }^{1}$, Nakanishi A. ${ }^{1}$, Horiuchi C. ${ }^{1}$, Tsuritani M. ${ }^{1}$, Iwanaga N. ${ }^{1}$, Kato Y. ${ }^{2}$, Sakaguchi H. ${ }^{2}$, Kurosaki K. ${ }^{2}$, Yoshimatsu J. ${ }^{1}$

National Cerebral and Cardiovascular Center, Perinatology, Osaka-Japan ${ }^{1}$

National Cerebral and Cardiovascular Center, Pediatric Cardiology, Osaka-Japan ${ }^{2}$

\section{Objective}

Long QT syndrome (LQTS) is a hereditary cardiac disease associated with a high risk of lifethreatening events such as syncope, ventricular tachycardia, and Torsades de Pointes. During the neonatal period, LQTS is one of the causes of sudden infant death syndrome (SIDS). It is known that the effective therapies as using $\beta$-blocker improve the outcome of patients with LQTS. Since the early diagnosis and treatment may help to prevent life-threatening events, early detection or suspicion of LQTS in utero is clinically important. We analyzed the associations with LQTS and fetal characteristics and evaluated the efficiency of cardiotocography (CTG) and fetal magnetocardiography (fMCG) for the prenatal diagnosis of LQTS.

\section{Methods}

Data were retrospectively analyzed using the medical records of women with LQTS who delivered between 2011 and 2019 at National Cerebral and Cardiovascular Center in Japan. Consequently, 31 pregnancies (25women) were included in the present study. According to the diagnosis of their infants, we classified into 2 groups as infants with LQTS (LQTS group) and infants without LQTS (non-LQTS). We investigated how the following data were vary between LQTS group and non-LQTS group; family history of LQTS, symptoms associated with LQTS, $\beta$-blocker usage, ICD implant, fetal heart rate (FHR) assessed by CTG, QT interval assessed by fMCG, emergency caesarian delivery, preterm birth, birth weight, arrhythmia of infant. We used CTG that recorded accurately over 30 minutes just before labor onset. Although MCG is performed in only a few centers, we are able to use it to assess the electromagnetic characteristics of fetal cardiac condition. We analyzed them to determine the associations with LQTS and fetal characteristics.

\section{Results}

Of 31 pregnancies, 7 cases were excluded because of the lack of final diagnosis of LQTS due to the insufficient examination. LQTS group accounted for $42.9 \%(n=13)$, non-LQTS group for $35.5 \%(n=11)$. No difference was observed between groups in family history of LQTS, symptoms associated with LQTS, $\beta$-blocker usage, ICD implant, emergency caesarian delivery, preterm birth, birth weight. The incidence of a reduced baseline FHR (110-120 bpm) assessed by CTG was significantly higher in LQTS group (LQTS group, $n=9$; non-LQTS group, $\mathrm{n}=1 ; \mathrm{p}<0.05)$. 14 fetuses whose mother didn't have ICD were performed fMCG and the incidence of prolongation of the QT interval (QTc $\geqq 480 \mathrm{msec}$ ) measured by fMCG was significantly higher (LQTS group, $n=4$; non-LQTS group, $n=0 ; p<0.05$ ).

\section{Conclusion}

In fetus recordings, a slightly reduced baseline FHR assessed by CTG and a prolongation of the QT interval assessed by fMCG was significantly associated with fetal LQTS. These findings reveled that CTG and fMCG are useful for the prenatal diagnosis of LQTS and the management of fetuses at risk of LQTS. 


\section{E1153 - THE CONTRIBUTION OF ECHOCARDIOGRAPHY IN THE NEWBORN EXPERIENCE OF THE NEONATOLOGY SERVICE}

Benrabah O. ${ }^{1}$, Ait Mouheb T. ${ }^{2}$

Hospital Parnet, Medicine, Hussein Dey-Algeria ${ }^{1}$

Hospiatal Belfort, Medicine, El Harrach Algeria ${ }^{2}$

Echocardiography in neonatology services has become an inescapable investigation for better management of certain clinical situations; including the suspicion of congenital heart disease, persistent fetal circulation or pulmonary arterial hypertension require the use of this examination.

\section{Objective}

To evaluate the contribution of echocardiography performed in the department.

\section{Methods}

We report through a prospective etiological cohort study, a series of 342 neonatal echocardiograms performed at the neonatology service level between January 2016 and December 2017.

\section{Results}

Congenital heart disease affects about 3.2 per 1000 live births. The main indications for echocardiography in the neonatology departments are: respiratory distress (10\%), O2 dependence $(24 \%)$, cyanosis (18\%). This anodyne and repetitive examination makes it possible to correct certain diagnoses, to confirm the presence of heart disease and sometimes to initiate vital treatments (prostaglandin if ducto-dependent heart disease), ventricular septal defect was the most frequent heart disease in our series $(22.5 \%)$ followed by atrial septal defect (13\%) and tetralogy of Fallot (10\%)

\section{Conclusion}

This work shows the possibilities of diagnosis and therapeutic adaptation offered by echocardiography in the neonatology services and especially the need to develop this act in front of any atypical cardiorespiratory evolution or the presence of risk factor. 
E1177 - COMPLEX FETAL CARDIAC MALFORMATION WITH ATYPICAL ASSOCIATIONS

Cirstoiu M. ${ }^{1}$, Turcan N. ${ }^{1}$, Teodor O. ${ }^{1}$, Baros A. ${ }^{1}$, Bohiltea . $^{1}$

University Emergency Hospital Bucharest Carol Davila University of Medicine and

Pharmacy, Bucharest, Obstetrics and Gynecology, Bucharest-Romania ${ }^{1}$

Congenital heart diseases are responsible for about 30-50\% of infant deaths. All severe, complex cardiac malformation can be partially or completely diagnosed prenatally. The prenatal diagnosis is crucial for both surgical managements postnatal or in utero in specialized centers or termination of pregnancy if the malformation is considered to be live incompatible. Double-outlet right ventricle is at the of the frequency list, having an incidence of $2-3 \%$ of all congenital heart defect. We present a case of an 18 years old gravida, 22 weeks of gestation which presents in the emergency service of the Bucharest Emergency University Hospital, directed form a provincial hospital for bradycardia and suspicion of fetal malformation. Emergency hospitalization was decided for monitoring and further investigations. The subsequent detailed echographic examination revealed a 22 weeks of gestation fetus with polyhydramnios and a complex cardiac malformation starting with dextrocardia and left isomerism. Apparently with a single right atrium and a hypoplastic left ventricle communicating with the right ventricle trough an important ventricular septal defect. Also, the transposition of the great vessels with an hypoplastic pulmonary artery emergent from the left ventricle was observed. On longitudinal-transversal view no image of the inferior vena cava could be noticed, only an enlarged azygos vein at the right, belonging to the same section plan, parallel with the aorta artery. Particularly, the fetus presented also an absent nasal bone and a ductus venosus agenesis. The patient never conducted any other pregnancy investigations. In the context of sever degradation of the fetal status and the complex associated malformations the termination of pregnancy was indicated and accepted by the patient. Further investigations are in course, respectively genetic assessment, considering that double-outlet right ventricle and conotruncal malformations have a risk of fetal aneuploidy association up to $40 \%$. 


\section{E1213 - ISOLATED PERSISTENT LEFT VENA CAVA SUPERIOR SYNDROME CASE REPORT}

Kavak S. ${ }^{1}$, Celik Kavak E. ${ }^{1}$, Sanli C. ${ }^{1}$, Seren O. ${ }^{2}$, Gulay B. ${ }^{3}$

Firat University Faculty of Medicine, Obstetrics and Gynecology, Elazig-Turkey ${ }^{1}$

Firat University, School of Medicine, Department of Obstetrics and Gynecology, Obstetrics and Gynecology, Elazig-Turkey ${ }^{2}$

Firat University, School of Medicine, Department of Obstetrics and Gynecology, Obstetrics and Gynecology, Elazig-Turkey ${ }^{3}$

We present a rare case of congenital cardiac anomaly, isolated persistent left vena cava superior, and discuss the prenatal diagnosis.

\section{Case}

A 37-year-old woman, G4P3, was admitted to our clinic due to the diagnosis of chronic hypertension. She did not know her last menstrual period. Her medical and family history revealed nothing except a suspicion of hypertension. There was no consanguinity with her husband. Blood pressure measurements were all under 140/90 mm hg. Ultrasonographic examination revealed a singleton fetus compatible with 23 weeks of pregnancy. Deepest amniotic fluid pocket was measured as $56 \mathrm{~mm}$. Cardiac examination revealed a normal four chamber view, inter ventricular septum, atrioventricular and semilunar valves. Foramen ovale flap was opening towards left. On the three vessels and trachea view, 4 vessels were observed and isolated vena cava superior diagnosis was done (Figure 1). Examination of the pulmonary veins revealed that two inferior pulmonary veins were opening to the left atrium. Dilated coronary sinus was observed on the atrial side of AV valves just under the 4 chamber view (Figure 2). Consultation was given to the patient with these findings. Spontaneous contractions started at 37 weeks and she gave birth vaginally. The diagnosis was confirmed on postpartum echocardiography. Newborn was discharged with recommendations.

\section{Conclusion}

Isolated persistent vena cava superior does not cause significant problems. However, if cardiac intervention is to be performed, the recognition of this situation earlier makes the procedure easier. 


\section{E1214 - SCIMITAR SYNDROME CONCERNING A CASE}

Ruiz García M. ${ }^{1}$, Bazán Legasa M. ${ }^{1}$, Barrenetxea Arrinda J. ${ }^{1}$, Zabaleta Loinaz I. ${ }^{1}$, Pedroarena Lecumberri I. ${ }^{1}$, Pérez Munárriz B. ${ }^{1}$, García Francés S. ${ }^{1}$, Urtasun Murillo M. ${ }^{1}$, Abián Franco N. ${ }^{2}$, Gastón Moreno B. ${ }^{1}$

Complejo Hospitalario De Navarra, Gynecologist and Obstetrics, Pamplona-Spain ${ }^{1}$

Hospital

Reina Sofía, Gynecologist and Obstetrics, Tudela-Spain ${ }^{2}$

\section{Introduction}

Partial anomalous pulmonary venous connection (PAPVC), encompasses a rare and complex group of congenital cardiovascular anomalies that are caused by the abnormal return of one or more, but not all, of the pulmonary veins directly to the right atrium or indirectly through a variety of venous connections from the anomalous pulmonary vein.

Scimitar syndrome is a variant PAPVC. Its defining feature is the anomalous pulmonary venous drainage of right lung, in which all (total form) or some (partial form) pulmonary veins drain into a systemic vein or into the right atrium rather than into the left one. The affected lung and its associated airways, which are drained by the scimitar vein, are often hypoplastic. Other cardiac defects are commonly seen, being typically present an atrial septal defect.

Considering the rareness of the syndrome and its unusual form of presentation, we present a case which may consider Scimitar syndrome as a differential diagnosis.

\section{Case}

24 year-old-woman undergoing her first spontaneous gestation. The course of pregnancy was normal until second trimester morphologic ultrasound when, despite the fact the exploration wasn't satisfactory enough due to the obesity of the patient, it was objectified dextrocardia. In this way, there were made several coming appointments to complete the study, visualizing right lung hypoplasia, some vessels anomalies and an echogenic mass lesion under the right lung. Afterwards, a magnetic resonance was performed and confirmed all these findings with the suspicion of Scimitar syndrome.

No other remarkable events occurred during the remaining pregnancy. At $40+2$ weeks a male fetus of 3690 gr was born by vacuum, Apgar score 8/9 and acid-base equilibrium of 7.15 7.23. No reanimation maneuvers were needed but the newborn was admitted to the neonatology unit to complete the study. There were performed several explorations: normal cerebral and abdominal ultrasound, an echocardiography verifying the previous discoveries. Moreover, a computed tomography was made showing dextrocardia, right lung hypoplasia with some vessels disorders, right pulmonary artery hypoplasia, inter-atrium connection and paravertebral eventration at right thoracic lower zone. So far, the neonate undergoes a well adaptation to the extra-uterine life except from his difficulty to gain weight and recurrent respiratory infections.

\section{Conclusion}

he initial diagnosis of PAPVC may be made by echocardiography and is typically confirmed by magnetic resonance imaging, computed tomography or cardiac catheterization. This syndrome has varied presentations, from an asymptomatic state to severe pulmonary hypertension and / or heart failure. Those who present early in life usually have associated congenital heart disease also. The outcome depends upon the underlying anatomy and the presence of associated conditions such as pulmonary hypertension, sequestration or hypoplasia. Symptomatic infants with this diagnosis tend to present with more severe disease and have a poorer prognosis than patients who present as adults or children. For this reason is essential to promptly diagnose this pathology in order to manage it as well as possible. 


\section{E1238 - NEONATAL AUTOIMMUNE MEDIATED CONGENITAL HEART BLOCK TWO CASE REPORTS}

Sfyril K. ${ }^{1}$, Makridi E. ${ }^{2}$, Sokou R. ${ }^{2}$, Krepi A. ${ }^{2}$, Karatzas K. ${ }^{2}$, Boutsikou T. ${ }^{2}$, Iliodromiti Z. ${ }^{2}$, Iacovidou N. $^{2}$

National and Kapodistrian University of Athens, Neonatal Department, Aretaieio Hospital, Athens-Greece ${ }^{1}$

National and Kapodistrian University of Athens, Neonatal Department, Aretaieio Hospital, Athens-Greece ${ }^{2}$

\section{Objective}

Congenital heart block (CHB) has an incidence of 1/15.000-20.000 births. Maternal risk factors include transplacental transfer of anti-nuclear antibodies to the fetus, metabolic diseases, medications, and viral infections. This transfer, may occur from 11 weeks of gestation, and represents the most common cause, known as autoimmune mediated CHB (ACHB). These autoantibodies attach to and subsequently harm the cardiomyocytes and conduction tissue in susceptible fetuses. We report 2 cases of neonates, who manifested CHB, born to asymptomatic mothers with positive ANA-antibodies.

\section{Case 1}

A 48-year-old primigravida following IVF was referred to our centre at 20+5 weeks of gestation age (GA), for fetal Echocardiography (ECHO), due to fetal bradycardia, noticed during routine obstetric ultrasound. ECHO findings, included systolic dysfunction of both ventricles, and complete CHB 2:1 with a ventricular rate of $70 \mathrm{bpm}$. Mother, who had been completely asymptomatic, underwent further work-up which revealed positive anti-SSA but negative anti-SSB antibodies. She was treated with low molecular weight heparin and oral dexamethasone 4mg/day. At 32+2 weeks, Doppler ultrasound revealed absence of enddiastolic flow velocity in the umbilical artery, severe fetal bradycardia, and symmetrical IUGR. A female neonate weighing 1320gr (<2nd centile) was born by Caesarean section (CS), with an Apgar score of 71 and 85. On admission to the NICU the neonate was intubated for respiratory distress. A dose of surfactant was administered and umbilical catheters were placed. The newborn was transferred to the reference center for congenital heart diseases with a heart rate of 65-70 bpm.

\section{Case 2}

A 40 year old (gravida: 5) was referred to the emergency department of our hospital because of fetal bradycardia (70-80 bpm) diagnosed on obstetric ultrasound at 20 weeks of gestation. She had a medical history of high titers, positive of Anti-ENA(++), Anti-Ro(++), and ANA(++) antibodies, without any clinical symptoms. During pregnancy medications included acetylsalicylic acid, hydroxychloroquine, and prednisolone. Fetal ECHO showed CHB; 2nd degree AV block and aortic stenosis hypoplasia, echogenic intracardiac focus, ventricular septal defect but no evidence of fetal distress. She was followed up by ultrasound scans on a weekly basis for the evaluation of fetal heart rate. At 38+6 weeks of gestation, CS was performed and a male neonate with birth weight of $2610 \mathrm{~g}$ (2nd centile), and an Apgar of 81 and 95, was born. At NICU, the newborn was intubated for respiratory distress and showed a heart rate of $50 \mathrm{bpm}$. Isoprenaline $0.02 \mathrm{~g} / \mathrm{kg} / \mathrm{min}$ was administered via UAC and the newborn was transferred to the reference center for congenital heart diseases for pacemaker placement. 


\section{Conclusion}

ACHB is undeniably a severe, potentially life-threatening disorder strictly defined as the presence of 2 key mandatory features (maternal anti-Ro/La auto-antibodies and a diagnosis of AV block in utero or postnatally). This helps differentiate ACHB from other non-autoimmune CHB which consists of different entities with an unknown and probably heterogeneous etiology. 
E1264 - A COMPLEX FETAL CARDIAC DISEASE RIGHT ATRIAL ISOMERISM

Kavak S. ${ }^{1}$, Celik Kavak E. ${ }^{1}$, Yener G. ${ }^{1}$, Sanlı C. ${ }^{1}$, Mirzaoğlu M. ${ }^{1}$

Firat University Faculty of Medicine, Obstetrics and Gynecology, Elazig-Turkey ${ }^{1}$

\section{Objective}

We present a rare case of right atrial isomerism detected at 23 weeks of gestation.

\section{Case}

A 26-year-old patient was referred to our clinic with a diagnosis of fetal cardiac anomaly. It was her first pregnancy. The patient's medical and family history were unremarkable. Her ultrasonographic examination revealed a 23 week old fetus which was consistent with the last menstruation period. Cardiac examination revealed a large AVSD (Figure 1). There was a flow towards right through the foramen ovale. On the 3 vessel trachea view a large vessel was shown in the center, a normally localized vena cava superior on the right and another vessel on the left (Figure 2). When ventricular outflow tracts were examined, a large aorta originating from the right ventricle and a thin pulmonary artery originating nearby was observed (DORV). The findings were consistent with pulmonary stenosis (Figure 3). When the area between the descending aorta and left atrium were examined, inferior pulmonary veins were not seen and confluens vein passing near the left atrium was observed (TAPVR). When atrial appendages were evaluated, both atria were found to be morphologically right atrium (Figure 4). Right atrial isomerism was considered with the present findings. The family was informed. A fetal MRI was requested. However, the family did not accept MRI and preferred the continuation of pregnancy. The baby was born at 34 weeks of pregnancy due to premature contractions. Diagnosis of the heart disease was confirmed after the birth. The infant was died in the neonatal intensive care unit on the 2nd day of birth.

\section{Conclusion}

Right atrial isomerism is a rare condition with poor prognosis. Detailed ultrasonographic examination is important for prenatal diagnosis and to give timely consultation to the family. 


\section{E1265 - A DOUBLE OUTLET RIGHT VENTRICLE CASE ACCOMPANYING PULMONARY STENOSIS DIAGNOSTIC CLUES}

$\underline{\text { Kavak S. }^{1}}$, Celik Kavak E. $^{1}$, Batmaz I. ${ }^{1}$, Keser B. ${ }^{1}$, Mirzaoğlu M. ${ }^{1}$

Firat University Faculty of Medicine, Obstetrics and Gynecology, Elazig-Turkey ${ }^{1}$

\section{Objective}

We herein report a double-outlet right ventricle case accompanying pulmonary stenosis, which is a rare cardiac anomaly and in which prenatal diagnosis is important.

\section{Case}

A 24 years old, G1P0, was referred to our hospital with the diagnosis of complex heart anomaly at 36. week of gestation. Her medical and family history revealed no abnormality and there was no consanguinity with her husband. It was learned that the patient had regular controls in pregnancy but did not underwent second trimester fetal ultrasound examination by an expert. A pregnancy compatible with 36 weeks was detected in the ultrasonography. Deepest amniotic fluid pocket was measured as $91 \mathrm{~mm}$ (mild polyhydramnios). The abdominal circumference was $303 \mathrm{~mm}$ and was consistent with 34 weeks of pregnancy. Cardiac examination showed atrioventricular septal defect and a slight shift to the right in the axis of the heart (Figure 1). The foramen ovale flap was opening towards left. Although the tricuspid valve appeared to be partially thickened, no significant weakness or stenosis was observed. On the three vessel trakea view, 2 vessels were seen and double-outlet right ventricle and pulmonary stenosis were detected (Figure 2-4). Examination of pulmonary veins revealed that 2 inferior pulmonary veins were opening to the left atrium (Figure 5). Consultation was given to the family and patient was delivered by cesarean section on the 37 . week of pregnancy due to start of spontaneous contractions. The diagnosis was confirmed on postpartum echocardiography. He was referred to cardiovascular surgeon for planning the treatment

\section{Conclusion}

It is important to establish the diagnosis of DORV in prenatal period for to deliver the baby in a tertiary clinic and to give appropriate counseling. 


\section{E1342 - MANAGEMET OF FETAL ARRHYTHMIA DURING DELIVERY}

Pedroarena I. ${ }^{1}$, Labayen A. ${ }^{1}$, Abián $N .{ }^{2}$, Ruiz M. ${ }^{1}$, Otaño J. ${ }^{1}$, Estefanía A. ${ }^{1}$

Complejo Hospitalario De Navarra, Department of Obstetrics and Gynecology, Pamplona Spain ${ }^{1}$

Hospital Reina Sofía, Department of Obstetrics and Gynecology, Pamplona-Spain ${ }^{2}$

Fetal arrhythmia appears in 1 to 2 percent of pregnancies. Some types of arrhythmia are associated with structural or functional heart disease, and may lead to compromise fetal circulation and ending to heart failure and hydrops. However, the most fetal arrhythmias are benign. For the screening assessment of the fetal heart rate, ultrasound four cardiac chambers correct and ventricular outflow tracts views may be sufficient. Some patients have higher risk to develop this pathology (pregnant women with anti-La and anti-Ro antibodies, antecedent of fetus with heart block or fetus with cardiac disease) and close follow-up should be recommended in these cases. The most common fetal arrythmia is the supraventricular tachycardia.

\section{Case}

A 28-year-old pregnant patient comes to the clinic for regular check-up in $40+1$ week of gestation. She did not have relevant medical or surgical background. This was her first pregnancy with normal evolution so far.

In this medical control an abnormal fetal cardiotocograph record (CTG) was detected. The obstetrician performed a fetal ultrasound, detecting fetal arrhythmia so she referred the patient urgently to the hospital.

In the hospital, the CTG showed fetal heart rate at $140 \mathrm{bpm}$, diminished variability and few transient increases so it was decided to end the pregnancy due to nonreassuring fetal status. A tolerance test for oxytocin was performed, resulting negative. Induction was continued with vaginal dinoprostone (PGE2). During delivery, there was a loss of variability in the fetal heart rate and few transient increases. With $3 \mathrm{~cm}$ of cervical dilatation, determination of fetal scalp $\mathrm{pH}$ was normal (7.30). An intrapartum fetal ultrasound was performed and ascites was detected suspecting heart failure, so an urgent caesarean section was indicated for nonreassuring fetal status.

The caesarean section proceeded uneventful. A 3280g girl was born. The amniotic liquid contained meconium. The determination of the umbilical cord artery and vein $\mathrm{pH}$ was normal (7.26 and 7.32 respectively). The APGAR at first and fifth minute of life was 9 and 10, respectively. The newborn was transferred to the Neonatal Intensive Care Unit (NICU) for close monitoring.

During her stay at the NICU, the newborn experienced episodes of supraventricular tachycardia at $290 \mathrm{bpm}$. The echocardiography showed decreased contractility, dominance of right cavities and atrial dilatation. Finally, it was decided to start treatment with flecainide at 3 $\mathrm{mg} / \mathrm{kg} /$ day with good evolution. As days go by echocardiography was normalized and the baby was discharged with ambulatory follow-up.

Vaginal delivery can be carried out in a fetus with arrhythmia as long as the control of fetal well-being can be properly performed. If fetal well-being cannot be evaluated adequately, delivery by caesarean section is the best option. It is also necessary a prepared neonatology team at the time of birth.

The supraventricular tachycardia rarely is mortal during delivery but the fetal heart rate must be corrected as soon as possible. 


\section{E1357 - AN EXREMELY RARE SEEN A CASE OF ECTOPIA CORDIS}

Yalinkaya A. ${ }^{1}$, Bozbay N. ${ }^{1}$, Elci E. ${ }^{1}$

Dicle University Medical Faculty, Obstetrics and Gynecology, Diyarbakir-Turkey ${ }^{1}$

The purpose of this study is a rare case of first-trimester diagnosis of ectopia cordis (EC). A 34 years old pregnant woman with gravida 3 parity 2 visited our clinic. According to last menstruation date, the patient was 13 weeks pregnant, she did not have any medical or obstetric pathology, she did not marry to a relative and she has not any use any medicine in history. Fetal cranium, face, neck, spine, thorax, abdominal front, stomach, kidney, bladder, upper and lower extremities were normal. Fetal nuchal translucence (NT) was $2.3 \mathrm{~mm}$. Fetal heart was outside the thorax rib cage and at normal rhythm. With family's consent, chorionic villus sampling has been made an option of termination given to the family. CVS result reported as a normal karyotype. At 14 weeks of her pregnancy patient terminated to another clinic.

Ectopia cordis is a congenital disease, characterized by hearts abnormal position than normal mediastinal location. During the embryogenesis a mistake with heart migration, heart partially or completely moves outside the thoracic cage. Babies with ectopia cordis can be diagnosed antenatally 10-12 weeks of pregnancy. Because ectopia cordis is extremely rare, we decided to do a case report. 


\section{E1368 - A REPORT OF TWO CASES PRENATALLY DIAGNOSED ABERRANTRIGHT SUBCLAVIAN ARTERY}

Demir S. ${ }^{1}$, Caglayan E. ${ }^{1}$, Altunyurt S. ${ }^{1}$

Dokuz Eylul University School of Medicine, Division of Perinatology, Department of

Obstetrics and Gynecology,Izmir -Turkey ${ }^{1}$

\section{Objective}

We aimed to present two cases which were diagnosed with aberrant right subclavian artery (ARSA) on detailed second trimester obstetric ultrasonography.

Case 1. A 23-year-old pregnant woman with G2P1 admitted to our perinatology outpatient clinic for routine detailed second trimester obstetric ultrasonography at her 20th gestational week. In the detailed fetal examination, fetal biometry was found to be consistent with 20 weeks and ARSA was detected by color doppler examination of three-vessel trachea section. Genetic counseling, about the presence of isolated ARSA and the accompanying chromosomal anomalies. Amniocentesis showed normal karyotype.

Case 2. A 25-year-old pregnant woman with G2P1 admitted to our perinatology outpatient clinic for routine detailed second trimester obstetric ultrasonography at 22 weeks of gestation. In the detailed fetal examination, fetal biometry was found to be consistent with 22 weeks and ARSA was detected by color doppler examination of three vessel trachea section. Ultrasonographic examination and fetal echocardiography showed no additional major anomaly except single umblical artery and ARSA. Genetic counseling was given to the patient who had low risk of first trimester screening test. Cordocentesis result was normal karyotype.

\section{Result}

Aberrant right subclavian artery (ARSA) is the most common aortic arcus anomaly or variation with a rate of $0.5-1.4 \%$ in the normal population. Normally, the right subclavian artery originates from the brachiocephalic artery (innominate artery), the first branch of the aorta at the level of the aortic arch. In the case of ARSA, four vessels emerge from the proximal to distal order, right main carotid artery, left main carotid artery, left subclavian artery and ARSA, respectively. The ARSA emerges from the distal part of the aortic arch and passes behind the esophagus and trachea to the right arm. The typical diagnosis of ARSA is not made on gray scala imaging. ARSA is best detected by color doppler in the three-vessel trachea image. The presence of ARSA in trisomy 21 fetuses was first reported by Chaoui. In subsequent studies, the frequency of ARSA in trisomy 21 fetuses was reported to be $14-30 \%$ and this relationship was confirmed. ARSA can be found in other aneuploidies. There is no consensus as to whether invasive procedures are required in the presence of isolated ARSA. Other anomalies accompany $20 \%$ of fetuses with ARSA. However, ARSA is an independent and important risk factor for trisomy 21. Recently, some centers have started to offer noninvasive prenatal test (NIPT) as an option in isolated ARSA cases.

Our first case was isolated ARSA without additional structural anomalies. The second case was ARSA with no additional structural anomalies other than single umbilical artery. In both cases, normal karyotype was detected after invasive procedure. Due to the possibility of coexistence of ARSA with other anomalies, detailed ultrasonographic evaluation and fetal echocardiography should be performed prior to defining it as isolated in the management, and it should be carefully evaluated whether there is any additional anomaly for fetuses diagnosed with ARSA and to provide detailed genetic counseling to the family about chromosome abnormalities will be appropriate. 


\section{E1375 - A CASE OF TUBEROUS SCLEROSIS DIAGNOSED WITH PRENATAL FETAL CARDIAC RHABDOMYOMA}

Demir . $^{1}$, Caglayan E. ${ }^{1}$, Altunyurt S. ${ }^{1}$

Dokuz Eylul University School of Medicine, Division of Perinatology, Department of

Obstetrics and Gynecology,, Izmir-Turkey ${ }^{1}$

\section{Objective}

To report a case of tuberous sclerosis diagnosed by fetal cardiac rhabdomyoma detected on detailed second trimester ultrasonography.

Case: A 29-year-old woman with G2 P1 Y1, 23 weeks and 3 days of gestation according to her last menstrual period was referred to our perinatology outpatient clinic due to fetal cardiac mass. The patient's antenatal screening tests were low-risk and there was no history other than previous cesarean section. In detailed fetal examination, an echogenic mass (Rhabdomyoma?) in the right atrium, in the right ventricle and in the left ventricle was observed (Figure 1). Fetal cranial MRI showed subependymal nodules in the periventricular white matter and lesions that were compatible with hamartoma in both kidneys (Figure 2). The patient underwent cordocentesis for prenatal diagnosis. Cytogenetic analysis and Tuberous sclerosis complex (TSC) gene panel were evaluated. Cytogenetic analysis in cordocentesis was evaluated as normal karyotype. The patient was diagnosed as tuberous sclerosis after TSC gene panel results (TSC 2 mutation). The karyotype analysis of the parents was evaluated as normal. In the routine follow-up of the patient, fetal bradycardia developed at the 35w6d of gestation, the patient was taken to emergency cesarean section operation and 2630 gr 5-7 APGAR baby girl was delivered. Bradycardia and cyanosis developed after delivery, she was admitted to the neonatal intensive care unit for follow-up and treatment. Postnatal control echocardiography: It was observed that cardiac rhabdomyomas did not cause obstruction of any valve or main vascular structures. The baby with good general condition and normal systemic examinations was discharged on the 15th day after delivery and was called for periodical follow-up visits by newborn pediatric cardiology, oncology, neurology, ophthalmology dermatology and genetic clinics.

\section{Conclusion}

Tuberous sclerosis complex (TSC); is a neurocutaneous syndrome with autosomal dominant inheritance that can affect multiple organ systems with low grade hamartomas (brain $~ 50 \%$, skin $\sim 50 \%$, heart $\sim 30 \%$, kidney $\sim 11 \%$ ). The incidence of the syndrome, first described by von Recklinghausen in 1862, is approximately one in 6000-10000 live births. In most cases, tuberous sclerosis is caused by mutations in the TSC1 (gene encoding hamartin) and TSC2 (gene encoding tuberin) genes on chromosomes 9 and 16, respectively. Newborns with TSC2 mutation have more severe disease and more severe mental retardation than those with TSC1 mutation. Approximately 2/3 of the cases has de novo mutations, while the rest are inherited from one of the parents. Prenatal diagnosis of tuberous sclerosis is possible by investigating TSC1 and TSC2 mutations in cases with positive family history and cardiac rhabdomyoma. Cardiac mass is the most important clinical finding for the diagnosis of tuberous sclerosis in intrauterine and neonatal period. The relationship between rhabdomyomas and tuberous sclerosis varies between $50 \%$ and $79 \%$. Since the risk of chromosomal abnormalities of cardiac rhabdomyomas is low, karyotyping is not mandatory. However, genetic counseling to parents is necessary to detect the presence of unknown, undiagnosed tuberous sclerosis in either. The most important finding leading to the diagnosis of tuberous sclerosis in intrauterine and neonatal period is the detection of 


\section{E1384 - RIGHT AORTIC ARCH WITH PATENT RIGHT DUCTUS WITH STRUCTURALLY NORMAL HEART A CASE REPORT}

Garg $P_{.}^{1}$, Baidwan A. ${ }^{2}$

Chaitanya Hospital, Obg, Chandigarh-India ${ }^{1}$

Chaitanya Hospital, Pead. Cardiology, Chandigarh-India ${ }^{2}$

Right Aortic arch refers to a congenitally abnormal position of aortic arch, i.e right of the trachea in 3VT view with or without abnormal branching pattern. Most commonly it is associated with left sided ductus which forms an abnormal U shaped appearance. Presence of right sided ductus with right aortic arch is less frequently documented, and is most commonly associated with other congenital heart diseases, eg TOF, Truncus arteriosus and Pulmonary atresia with ventricular septal defect. Structurally normal heart with right sided aortic arch and right sided ductus is uncommon, clue to diagnosis in foetal ECHO is presence of normal " $v$ " shaped confluence of aortic arch and ductal arch in 3VT view with trachea right of the ductal arch. A 33 year old second gravida, with no antenatal risk factor was found to be having structurally normal heart, and on cranial sweep both ductal arch and trachea were located right of Trachea. To conclude, right aortic arch with right ductus can be associated with structurally normal heart. 


\section{E1478 - CARDIAC AXIS MEASUREMENT DURING THE FIRST TRIMESTER SCREENING ULTRASOUND}

Kesrouani A. ${ }^{1}$, Nasr B. ${ }^{2}$, Daou L. ${ }^{3}$, Hachem R. ${ }^{1}$, Hatoum I. ${ }^{4}$ Saint Joseph University, Obstetrics and Gynecology, Beirut-Lebanon ${ }^{1}$

Fetal Care Center, Fetal Medicine, Beirut Lebanon ${ }^{2}$

Saint Joseph University, Ardiopediatrics, Beirut-Lebanon ${ }^{3}$

Rafic Hariri University Hospital, Obstetrics and Gynecology, Virginia-Lebanon ${ }^{4}$

\section{Objective}

Cardiac axis (CAx) measurement at the end of the first trimester has been reported as feasible and could potentially screen for cardiac pathology. In this pilot study in a Lebanese population we aim to describe the distribution of CAx measurements as noted for the Crown Rump length measurement (CRL) during the first trimester scan.

\section{Methods}

The population studied consisted of 100 consecutive patients consulting for the first trimester screening ultrasound. CRL and NT were routinely assessed as defined by the guidelines by a single operator. An abdominal or vaginal approach was used according to the presentation of the fetus. The images for the 4-chamber view were defined when a single full rib was visible on each side of the fetal lateral chest wall and clear visualization of the heart attaining one-third of the display screen. Color Doppler was eventually used to confirm the location of the septum and the fetal spine was situated at the 6 or 12 o'clock position. The angle between the line for the septum and the anteroposterior line was taken. Measurements were taken twice in case the operator felt it wasn't clear enough and the mean was recorded then. We excluded from the study patients with abnormal NT, cases with abnormalities found during ultrasound and cases who were suspected for cardiopathy during follow-up.

Statistical analysis was performed with Medcalc software. This study was approved by our institution's ethics committee.

\section{Results}

Our series included 100 patients with a mean age of 31 years. NT was below the 95th percentile in all of these patients. In all cases the cardiac axis angle could be measured. Two cases were excluded from the study, one because of the diagnosis of trisomy 21 after NIPT screening and the other for a septal defect diagnosed at 21 weeks. Our series included then two additional patients to reach the 100 patients number. The mean for CAx is $48^{\circ}$, ranging from 39 to $60^{\circ}$, with a standard deviation of 5.2. The 2.5 percentile was defined at $40^{\circ}$ and the 97.5 percentile at $59^{\circ}$. the distribution of the CAx according to the CRL is reported in Figure 1 along with the confidence interval for each CRL measurement. Figure 2 represents the CAx as a z-score. Higher CRL was associated with a trend of a decrease of the CAx.

\section{Conclusion}

Measuring cardiac axis is feasible in all the cases included in this pilot study. A mean of $48^{\circ}$ $+/-5.2$ is noted. Larger series including pathological and normal cases could help discriminate values that could lead to cardiopathy diagnosis. 


\section{Obstetrics - Diabetes and obesity during pregnancy}

\section{E1074 - THE ROLE OF EXCLUSIVE BREASTFEEDING IN IMPROVING OGTT RESULTS OF PATIENTS WITH GESTATIONAL DIABETES MELLITUS AT SIX TO TWELVE WEEKS POSTPARTUM}

Rivera L. ${ }^{1}$, Jacquiline M. ${ }^{1}$

San Juan De Dios Educational Foundation, Inc. (hospital), Ob-gyn, Las Pinas City Philippines $^{1}$

\section{Objective}

Gestational Diabetes Mellitus (GDM) is one of the most common diseases complicating pregnancy and is associated with both maternal and fetal complications. Women with GDM have a high risk of developing postpartum type II diabetes mellitus. Prevention of this progression is speculated to be influenced by lifestyle modifications including breastfeeding, diet, and exercise. The objective of this study was to determine if exclusive breastfeeding during the postpartum period can improve results of 75 gram OGTT done at 6-12 weeks postpartum.

\section{Methods}

We compared baseline and postpartum 75 gram OGTT results of 120 patients with GDM in a tertiary hospital. Comparison was done among four (4) subgroups: (a) those that did not breastfeed, (b) those who breastfed for 1-4 weeks, (c) those who breastfed for 5-8 weeks, and (d) those who breastfed for 9-12 weeks. Analysis of variance (ANOVA) was used to compare the means of the four subgroups versus their 75 grams OGTT results.

\section{Results}

There was a significant difference in 75 gram OGTT results done at 24-28 weeks age of gestation and done 6-12 weeks postpartum, for values of fasting blood sugar, first hour, and second hour. There was also an observed higher decline in values as duration of exclusive breastfeeding increases.

\section{Conclusion}

This study showed that exclusive breastfeeding during the postpartum period is associated with improvement of results of 75 gram OGTT done at 6-12 weeks postpartum compared with those done at 24-28 weeks age of gestation. It was also observed that duration of breastfeeding is inversely correlated with 75 gram OGTT values. 


\section{E1087 - THE ROLE OF EXCLUSIVE BREASTFEEDING IN IMPROVING OGTT RESULTS OF PATIENTS WITH GESTATIONAL DIABETES MELLITUS AT SIX TO TWELVE WEEKS POSTPARTUM}

Rivera L. ${ }^{1}$, Marcos J. ${ }^{2}$

San Juan De Dios Hospital, Dept.of Obstetrics and Gynecology, Manila-Philippines ${ }^{1}$

San Juan De Dios Hospital, Ept.of Obstetrics and Gynecology, Pasay City-Philippines ${ }^{2}$

\section{Objective}

Gestational Diabetes Mellitus (GDM) is one of the most common diseases complicating pregnancy and is associated with both maternal and fetal complications. Women with GDM have a high risk of developing postpartum type II diabetes mellitus. Prevention of this progression is speculated to be influenced by lifestyle modifications including breastfeeding, diet, and exercise. The objective of this study was to determine if exclusive breastfeeding during the postpartum period can improve results of 75 gram OGTT done at 6-12 weeks postpartum.

\section{Methods}

We compared baseline and postpartum 75 gram OGTT results of 120 patients with GDM in a tertiary hospital. Comparison was done among four (4) subgroups: (a) those that did not breastfeed, (b) those who breastfed for 1-4 weeks, (c) those who breastfed for 5-8 weeks, and (d) those who breastfed for 9-12 weeks. Analysis of variance (ANOVA) was used to compare the means of the four subgroups versus their 75 grams OGTT results.

\section{Results}

There was a significant difference in 75 gram OGTT results done at 24-28 weeks age of gestation and done 6-12 weeks postpartum, for values of fasting blood sugar, first hour, and second hour. There was also an observed higher decline in values as duration of exclusive breastfeeding increases.

\section{Conclusion}

This study showed that exclusive breastfeeding during the postpartum period is associated with improvement of results of 75 gram OGTT done at 6-12 weeks postpartum compared with those done at 24-28 weeks age of gestation. It was also observed that duration of breastfeeding is inversely correlated with 75 gram OGTT values. 


\title{
E1132 - GESTATIONAL DIABETES MELLITUS EARLY DIAGNOSIS
}

\author{
Epishkina-Minina A. ${ }^{1}$, Khamoshina M. ${ }^{1}$
}

People's Friendship University of Russia (RUDN University), Department of Obstetrics and

Gynecology with a Course of Perinatology of Medical Institute, Moscow-Russia ${ }^{1}$

\section{Introduction}

Gestational diabetes mellitus (GDM) is an interdisciplinary problem. The important medical and social significance of this disease is determined by the high frequency of adverse pregnancy outcomes, both for the mother and for the newborn due to a significant increase in the likelihood of various complications of gestation.

\section{Objective}

To determine the risk predictors of GDM and develop a model of mathematical prognosis of the disease risk, suitable for use in the first trimester of pregnancy.

\section{Methods}

The present study was cohort and prospective. The study and control groups were formed from among 1140 women who stood up in 2018 on the account of pregnancy up to 12 weeks of gestation in the women's consultation in Moscow and were under observation throughout the pregnancy from the time of registration to childbirth. In accordance with including criteria, 357 women were selected. Fasting glucose, glycated hemoglobin, serum iron and hemoglobin levels were determined in patients at 7-10 weeks of gestation. Two groups of patients were distinguished: with developed GDM $(n=32)$ and control group $(n=61)$. The role of markers of tissue hypoxia and Hba1c as predictors of gestational diabetes mellitus was evaluated.

\section{Results}

The study materials were statistically processed using the methods of parametric and nonparametric analysis. The accumulation, correction, systematization of initial information and visualization of the results were carried out in Microsoft Office Excel 2016 spreadsheets. Statistical analysis was performed using IBM SPSS Statistics V. 23 (developed by IBM Corporation). Based on the identified factors that have a statistically significant relationship with the presence of GDM, a prognostic model was developed using the binary logistic regression method: $\mathrm{P}=1 /(1+\mathrm{e}-\mathrm{z}) * 100 \%, \mathrm{z}=-54,8+0,19 *$ Xage $+0,89 * \mathrm{XBMI}+4,3 * \mathrm{Xglu}$ $+5,92 *$ XHbAc1 $-0,2 *$ XserI $-0,17 *$ Xhem, where $\mathrm{P}$ is the probability of the presence of GDM (\%), Xage is the age of the woman (full years), XBMI is the body mass index $(\mathrm{kg} / \mathrm{m} 2)$, Xglu is the serum glucose (mmol/l), XHbAc1 is the level of glycated hemoglobin (\%), XserI is the serum iron content in the blood $(\mathrm{ng} / \mathrm{ml})$, XHem is the hemoglobin content in the blood (g/l).

The obtained prognostic model was statistically significant $(\mathrm{p}<0.001) .50 \%$ was used as a separating value - with a value of $\mathrm{P}$ equal to or above $50 \%$, a high risk of GDM was recognized, with values below $50 \%$ - a low risk of GDM.

\section{Conclusion}

In the modern population of healthy Moscow women of optimal reproductive age, the probability of GDM verification is $9 \%$. The risk group is formed by women who have at the time of registration of $\mathrm{BMI} \geq 24.4 \mathrm{~kg} / \mathrm{m} 2$ and the fact of determining in the first trimester levels of glycated hemoglobin $\geq 4.85 \%$, hemoglobin $\leq 109.5 \mathrm{~g} / 1$ and serum iron $\leq 10.7 \mathrm{ng} / \mathrm{ml}$. the proposed mathematical model can be used for mathematical prediction. 


\section{E1139 - DIABETES INSIPIDUS AND GESTATIONAL DIABETES IN PREGNANCY AND POSTPARTUM}

Mocuta D. ${ }^{1}$, Aur C. ${ }^{1}$, Copos A. ${ }^{1}$, Mascas G. ${ }^{1}$, Cuc R. ${ }^{1}$

County Clinical Emergency Hospital of Oradea, Obstetrics and Gynecology, Oradea

Romania $^{1}$

Diabetes insipidus (DI) is a condition characterized by large amounts of dilute urine and increased thirst. The amount of urine produced can be nearly 20 liters per day. Reduction of fluid has little effect on the concentration of the urine. There are four types of DI, each with a different set of causes. Central DI is due to a lack of the hormone vasopressin (antidiuretic hormone), because a damage to the hypothalamus, pituitary gland or genetics. Nephrogenic diabetes insipidus occurs when the kidneys do not respond properly to vasopressin. Dipsogenic DI is due to abnormal thirst mechanisms in the hypothalamus. Gestational DI occurs only during pregnancy or postpartum period, because during pregnancy, women produce vasopressinase in the placenta, which breaks down antidiuretic hormone (ADH). Gestational DI is thought to occur with excessive production and/or impaired clearance of vasopressinase. Diagnosis is often based on urine tests, blood tests, and the fluid deprivation test. In diabetes mellitus there is a production of large amounts of urine too, that's why is necessary to make differential diagnosis these two types of diabetes. We present here a case of a 30 years old pregnant woman IIGIIP, diagnosed with gestational diabetes and diabetes insipidus in second pregnancy. First pregnancy was finalized at 26 weeks with a C-section for abruptio placentae, but the 700 gr premature baby survived only 4 days. With second pregnancy she was monitorised from first trimester and at 27 weeks of amenorrhea diagnosed with gestational diabetes. She was took in evidence in diabetology clinic and put on Insulin therapy because increased glycemic values. She had also poliuria, polidipsia in the last 4 weeks of pregnancy. She delivered by C-section a healthy $2700 \mathrm{gr}$ baby at 37 weeks of amenorrhea, after premature rupture of membranes. In postpartum she presented a symptomatology that suggests diabetes insipidus. At 36 hours after the intervention, started the poliuria, the amount of urine being bigger than the quantity of ingested or administrated fluids, $11 \mathrm{l} / 24$ hours in 2 consecutive postpartum days. For differential and etiologic diagnosis were solicitated multidisciplinary consults- endocrinology, nephrology, and diabetology. The glycemic levels were controlled with difficulty, the lactic acid levels were variable too for few days, also the diuresis which decreased slowly in 10 days at $3000 \mathrm{ml} / 24 \mathrm{~h}$.

In our case it was difficult to establish correct diagnosis and management because the patient was non-cooperative, didn't communicate to her doctor about her previous symptoms to prevent the postpartum situation with large variations in hard-to-adjust glycaemia, short acidosis states and large amount of urine. However, her general condition over the day was good, including breastfeeding her baby.

It is a rare situation- pregnant patient with gestational diabetes and diabetes insipidus and very important is the communication doctor-patient and a multidisciplinary medical cooperation for a correct therapeutically management. 


\section{E1140 - POSTNATAL CARE AND FOLLOW UP IN GESTATIONAL DIABETES}

Mocuta D. ${ }^{1}$, Aur C. ${ }^{1}$, Copos A. ${ }^{1}$, Mascas G. ${ }^{1}$, Cuc R. ${ }^{1}$

County Clinical Emergency Hospital of Oradea, Obstetrics\&Gynecology, Oradea-Romania ${ }^{1}$

\section{Objective}

Gestational diabetes-GD-represents any hyperglycemia diagnosed during pregnancy that was not clearly overt diabetes prior to gestation. Hyperglycemia in pregnancy is associated with adverse maternal and prenatal outcome, that's why is important to screen, diagnose and treat hyperglycemia in pregnancy. All women with GD should be tested in postpartum period, not earlier than 4-12 weeks after delivery, in order to detect the risk for developing type 2 diabetes. Insulin sensitivity increases in the following days after delivering the baby and the placenta, so it is returning to the pre-pregnancy levels in the next 2 weeks. The frequency for testing is every 1-3 years, according with individual risk factors like high BMI, family history, insulin dependent form of gestational diabetes.

\section{Methods}

Our study group consists of 50 patients, diagnosed with GD and delivered in our department in the last 2 years (27 cases in 2017 and 23 in 2018). In 3 of the cases, insulin therapy was required for a good glucose control. They were screened during the postnatal care, at 4-12 weeks, by OGTT with $75 \mathrm{~g}$ glucose and by HbA1c level measurement, in the same day. Results were analyzed using criteria for no pregnant no diabetic women. In patients with test results near the margins range, tests were repeated after 3-6 months.

\section{Results}

On the basis of abnormal OGTT and/or HbA1c results, recommendations for changes in lifestyle, diet control and exercising were made, in order to decrease the incidence of type 2 diabetes later in life. For our patients, in the most cases, the results at the 6 months and then at 12 months after delivery showed good blood sugar levels. From the 3 patients which needed insulin therapy, 1 patient presented later good glycemia levels in postpartum only with diet control, 1 needed oral anti-diabetic therapy and the last one has remained on insulin therapy.

\section{Conclusion}

Because of the increased lifetime maternal risk for type 2 diabetes in women with gestational diabetes, they should be follow-up by laboratory tests. Screening and diagnosis of GDM and treating it effectively not only prevent adverse maternal and perinatal outcome but also future diabetes in both mother and child. This method of investigation OGTT, is simple in execution, patient friendly, accurate in diagnosis and easy to be performed by any laboratory. 


\section{E1180 - PREGNANCY OUTCOME IN GENITAL TRACT INFECTION ASSOCIATED WITH DIABETES}

Aur C. ${ }^{1}$, Mocuta D. ${ }^{1}$, Cuc R. ${ }^{1}$, Copos A. ${ }^{1}$, Mascas G. ${ }^{1}$

University of Oradea, Clinical Emergency Hospital of Oradea Obstetrics and Gynecology,

Obstetrics and Gynecology, Oradea-Romania ${ }^{1}$

\section{Objective}

It is well known the high maternal and fetal risk for complications in pregnancy associated with diabetes. Genital tract infection has a more increased incidence in pregnancy associated with diabetes. The goal of our study was to assess the frequency and the type of complications which can appear at the pregnant patients who were diagnosed with diabetes and antepartum genital tract infection.

\section{Methods}

This is a retrospective study, about the pregnant women with preexistent or gestational diabetes who delivered in our obstetric department in a period of 24 months (1st of January 2017 - 31st of December 2018). Vaginal secretion was sampled from all the patients and microbiological study was performed. The pregnancy outcome was analyzed in relation with the presence or the absence of genital tract infection.

\section{Results}

During the last 2 years, 7194 women delivered in our hospital. From these, 48 cases were monitored during pregnancy because of their high glycemic values. In this group, for 17 patients the diabetologist recommended insulin therapy. Among pregnant women with diabetes admitted in the delivery room, abnormal vaginal flora was identified at 27 patients, which means more than half of them. Escherichia Coli was present in 19 cases (39,58\%), Klebsiella pneumoniae, Proteus mirabilis and Gardnerella vaginalis appeared each in 2 cases (4,16\%). Group B Streptococcus and Enterobacter appeared each of them in 1 case $(2,08 \%)$. Most of the cases had candidiasis, too.

Cases with diabetes and genital tract infections had a high incidence of material and fetal complications comparing with cases without infection. The most frequent complications we can mention are: premature births in 15 cases (31,25\%), retroplacental hematoma in 11 cases $(22,91 \%)$ and the delivery finalized by C-section in 43 cases (89,58\%). Neonatal complications included hypoglycemia, hyperbilirubinemia, hypocalcemia and neurological damage especially in the premature new born babies (in 2 cases).

\section{Conclusion}

Genital tract infection in patients with diabetes was highly associated with poor perinatal outcome. It is very important to perform the microbiological screening in these pregnancies. Controlling the genital infection and the level of glycemia in these patients could improve the outcome. 
E1208 - RISK FACTORS FOR DEVELOPMENT OF GLYCEMIC ANOMALIES IN THE POST PARTUM PERIOD A SECONDARY ANALYSIS OF THE SOUTH BELGIAN DIABETES IN PREGNANCY STUDY (BEDIP S)

Costa E. ${ }^{1}$, Gerday C. ${ }^{1}$, Galopin C. ${ }^{1}$, Kirkpatrick C. ${ }^{1}$, Scheen A. ${ }^{2}$, Philips J. ${ }^{2}$,

Daelemans C. ${ }^{1}$, Pintiaux A. ${ }^{1}$

Ulb, Obgyn, Brussels-Belgium ${ }^{1}$ Ulg, Endocrinology, Liege-Belgium ${ }^{2}$

\section{Objective}

To establish which risk factors among clinical, socioeconomic and biological are associated with the development of glycemic anomalies 3 months post-partum (PP).

\section{Methods}

This study was performed on the cohort of the BEDIP-S study. We recruited healthy patients carrying singleton pregnancy, screened them for gestational diabetes (GD) with both IADPSG and Two step ADA 2003 criteria and treated according to IADPSG criteria only. Three months PP we prescribed, to all GDM subjects and a subgroup of normoglycemic subjects who agreed to further testing, a screening for glycemic anomalies with a 2 hour $75 \mathrm{~g}$ OGTT.

\section{Results}

Of the 1006 patients included, only 99 patients completed the study until PP, of which 10 had glycemic anomalies in the PP period according to WHO out of pregnancy criteria. Those who were positive in the PP period had a statistically significantly higher BMI (24.6 vs 28.3, $\mathrm{p}=0.017$ ), had had a higher number of previous pregnancies ( 2 vs $3.5, \mathrm{p}=0.016$ ) were more often of middle eastern-north African ethnicity ( $24.2 \%$ vs $60 \%, p=0.016$ ) had lower number of revenues in the family ( 20.65 vs $60 \%$ of families with one revenue or less, $p=0.022$ ). From a biological point of view they had a significantly higher amount of fasting triglycerides at first trimester ( 80.2 vs $127.3, \mathrm{p}<0.001$ ), were more likely to have been diagnosed with GDM according to IADPSG criteria (32\% vs $70 \%, \mathrm{p}=0.032$ ) but not with two step criteria $(p=0.346)$. Newborns were more likely to have experienced shoulder dystocia (3.4\% vs $20 \%$, $\mathrm{p}=0.025$ ) and had higher birthweight percentile (52th centile vs 85th centile, $\mathrm{p}=0.025$ )

\section{Conclusion}

Detecting glycemic anomalies in the PP period is paramount in order to intervene and avoid cases of type 2 diabetes in the future. Compliance to PP OGTT is low, as shown by the low number of patients that completed the study, even among GDM positive patients. Apart from the presence of GDM, we singled out other risk factors for PP glycemic anomalies (BMI, higher number of previous pregnancies, ethnicity, only one revenue in the family, triglycerides at first trimester, shoulder dystocia at the moment of birth and higher birthweight centile). Those could help targeting a subpopulation for whom the completion of a PP OGTT is particularly important. 
E1230 - DIABETIC KETOACIDOSIS AS THE ONSET OF DIABETES MELLITUS IN A YOUNG PREGNANT WOMAN A CASE REPORT

Bazan M. ${ }^{1}$, Barrenetxea J. ${ }^{2}$, Zabaleta I. ${ }^{3}$, Pedroarena I. ${ }^{1}$, Perez B. ${ }^{1}$, Garcia S. ${ }^{1}$, Urtasun

$\mathrm{M}^{3}$, Abian N. ${ }^{4}$, Gaston B. ${ }^{4}$, Ruiz M. ${ }^{1}$

Complejo Hospitalario De Navarra, Gynecology and Obstetrics, Pamplona-Spain ${ }^{1}$

Hospital De Urduliz, Gynecology and Obstetrics, Urduliz-Spain ${ }^{2}$

Complejo Hospitalario De Navarra, Gynecology and Obstetrics, Pamplona-Spain ${ }^{3}$

Hospital Reina Sofia, Gynecology and Obstetrics, Zizur Mayor-Spain ${ }^{4}$

\section{Introduction:}

Diabetic ketoacidosis is one of the most serious complications of diabetes, and it is defined by the presence of all the following in a patient with diabetes: ketosis (presence of ketones in blood or urine), hyperglycemia ( $>200 \mathrm{mg} / \mathrm{dL}$ ) and metabolic acidosis (venous $\mathrm{pH}<7.3$ or serum bicarbonate $<15 \mathrm{mEq} / \mathrm{L}$ ).

Physiological changes and pathological conditions related to pregnancy predispose women with diabetes to worsening glycemic control. Despite this, diabetic ketoacidosis (DKA) occurs in only about 0.5 to 3 percent of diabetic pregnant women.

\section{Case}

We report the case of a 18-year old woman, 29+1 weeks of pregnancy who comes to the emergency room of our hospital with intense nausea and vomiting, without other systemic or obstetric symptoms. She reported normal fetal movements and normal gestational course until the moment. This was her first pregnancy, and she had no history of previous diseases except from obesity. Nevertheless, she had a large family history of type-2 diabetes. The results of the blood tests showed severe hyperglycemia $(518 \mathrm{mg} / \mathrm{dL})$ along with hemoconcentration parameters and elevated serum creatinine $(1,7 \mathrm{~g} / \mathrm{dL})$, and without any sign of an active infection. She was monitored and we started endovenous insulinotherapy as the main suspicion was a diabetic ketoacidosis. The initial CTG showed fetal tachycardia (165 $\mathrm{lpm}$ ) with a reactive pattern. As soon as the patient was stabilized the fetal heart rate became normal and she reported a clinical improvement. She stayed in our hospital for 5 days. During this time, she was performed more blood tests in order to try to reach a precise diagnosis. She had normal C-peptide levels as well as high levels of HbA1c (10.6\%), which suggested the diagnosis of type 2 diabetes. After 5 days of hospitalization with high doses of subcutaneous insulin she was discharged from our hospital with recommendations, treatment and follow-up consultations in High Risk Pregnancy consultation and Endocrinology.

\section{Conclusio}

The presentation of DKA is similar in pregnant women to that in nonpregnant people, with symptoms of nausea, vomiting, thirst, polyuria, polydipsia, abdominal pain, and, when severe, a change in mental status.

Severe hyperglycemia can cause an osmotic diuresis resulting in maternal volume depletion. This, in turn, can result in reduced uterine perfusion and, in association with the metabolic abnormalities of DKA, produce life-threatening fetal hypoxemia and acidosis, as well as renal failure. Maternal mortality is less than 1 percent, but fetal mortality rates of 9 to 36 percent have been reported, as well as increased risks of preterm birth. Thus, DKA is a true obstetrical emergency.

Other than close attention to fetal heart rate monitoring, DKA is managed similarly in pregnant and nonpregnant patients. This includes the use of intravenous insulin, volume replacement, monitoring acidosis, and a search for precipitating causes, such as infection or insulin noncompliance. 
DKA alone is generally not an indication for delivery. Emergency delivery before maternal stabilization should be avoided because it increases the risk of maternal morbidity and mortality, and may result in delivery of a hypoxic, acidotic preterm infant for whom in utero resuscitation may have resulted in a better outcome. 


\section{E1274 - PATHOLOGICAL FEATURES OF PLACENTAL ABRUPTION IN MATERNAL DIABETES}

Aur C. ${ }^{1}$, Popescu E. ${ }^{2}$, Mocuta D. ${ }^{1}$, Copos A. ${ }^{1}$, Cuc R. ${ }^{1}$, Mascas G. ${ }^{3}$

County Emergency Clinical Hospital, Ob-gyn Department, Oradea-Romania ${ }^{1}$

County Emergency Clinical Hospital, Histopathology Department, Oradea-Romania ${ }^{2}$

County Clinical Emergency Hospital Of Oradea, Ob-Gyn Department, Oradea-Romania ${ }^{3}$

\section{Objective}

Placental abruption is one of the most dangerous conditions in pregnancy, life threatening for the mother and the child as well. Hypertensive disorders and diabetes associated with pregnancy are considered major risk factors for placental abruption. Our study goal is to assess the link between the histopathological features and the perinatal outcome in cases of diabetes- associated pregnancies complicated with placental abruption.

\section{Methods}

We completed a retrospective study of all the cases of maternal diabetes who delivered in our tertiary university obstetric department during a period of 24 months (between 1st of January 2017 and 31stof December 2018). Placental abruption was diagnosed in 11 from the 48 cases $(22,9 \%)$. In only 3 of the cases the diagnosis was established before the delivery and the decision was the emergency cesarean section. Because of the systematic examination of the placentas after deliveries, the obstetrician described the presence of the retroplacental hematoma or blood clot in the other 8 cases, all delivered by elective cesarean section. The macroscopic and microscopic description of the placental changes was made after the histopathological examination. The condition of the babies was assessed by the Apgar Score. We systematically recorded from the hospital charts the significant clinical data and the pathological findings.

\section{Results}

Patients with placental abruption and diabetes had a history of cesarean section in 4 cases. No one of the babies died in utero in our study group. Fetal heart rate abnormalities appeared in 5 of the cases. All the babies had a good Apgar Score and a favorable outcome. Placental abnormalities were registered with a wide range of variation. The weight of the placenta was higher than those in nondiabetic pregnant women. The placentas were severe abnormal, enlarged, thick, plethoric in cases with pre pregnancy diabetes (in 3 cases). Unusually thick decidua, dysmature villous structure, villous hypervascularity or even chorangiosis were present in almost all cases with poor glucose control. The most frequent placental pathologic nonspecific features were meconium staining, acute atherosis, fibrinoid necrosis, villous oedema or fibrosis.

\section{Conclusion}

Diabetes is highly associated with placental abruption. Surprising was the good outcome of all the babies delivered in case of diabetes complicated with placental abruption in our group. That could mean that the wellbeing of the fetus was not affected by the pathological changes described in these placentas. Histopathological examination of the placenta following pregnancies affected by complications may be an important tool in the patient care. Taking in consideration this information, the management of the subsequent pregnancies could also be improved. 


\section{E1371 - EFFECTS OF WEIGHT ON BIOAVAILABILITY OF MISOPROSTOL DURING INDUCTION OF LABOR IN PREGNANT WOMEN WITH OBESITY}

Filgueira G. ${ }^{1}$, Nardoto G. ${ }^{2}$, Marques M. ${ }^{2}$, Filgueira O. ${ }^{1}$, Carvalho D. ${ }^{1}$, Moises E. ${ }^{1}$, Duarte G. ${ }^{1}$, Lanchote V. ${ }^{2}$, Cavalli . $^{1}$

Ribeirão Preto Medical School, University of São Paulo, Gynecology and Obstetric, Ribeirão Preto-Brazil ${ }^{1}$

Faculty of Pharmaceutical Sciences Of Ribeirão Preto, University of São Paulo, Clinical Toxicologic and Bromatologic Analyses, Ribeirão Preto-Brazil ${ }^{2}$

\section{Objetive}

The aim of the study was to evaluate the influence of weight on the area under the curve (AUC) of misoprostol (misoprostol acid) and consequently in rates of labor induction failure of pregnant women with obesity.

\section{Methods}

We investigated 40 pregnant women distributed as Group 1: 10 non-obese, Group 2: 10 with obesity grade 1, Group 3: 10 with obesity grade 2 and Group 4: 10 with obesity grade 3 . After admission of the pregnant women, reading and signing the Informed Consent Term, the length of cervix was evaluated, the Bishop index was determined and blood samples were collected for evaluation of the laboratory tests and zero time of pharmacokinetics. Following administration of the $25 \mu \mathrm{g}$ misoprostol vaginal tablet, serial blood samples were collected at times of 15 to 360 minutes. Plasma concentrations of misoprostol acid were analyzed in plasma using UPLC-MS/MS. Pharmacokinetic parameters were calculated based on the total plasma concentration versus time curves using the Winnolin program. The Kruskal-Wallis test was used and as post-test was used the Dunn test for statistical analysis.

\section{Results}

All pregnant women performed prenatal satisfactorily and none had undergone previous caesarean section. There was a significant difference in labor induction failure between groups. The rate of induction failure in Group 4 was $70 \%$, while in the other groups it was only $10 \%$. The method for the analysis of misoprostol acid in plasma followed the prevailing legislation, with lower limit of quantification (LIQ) of $2.0 \mathrm{pg} / \mathrm{mL}$. The median of AUC0- $\infty$ for Group 1 iwas 83.89 pg/mL, for Group 2 was $73.93 \mathrm{pg} / \mathrm{mL}$, for Group 3 was $68.84 \mathrm{pg} / \mathrm{mL}$ and for Group 4 was $56.77 \mathrm{pg} / \mathrm{mL}$. There was weight influence on the AUC (p-value=0.0089) (Figure 1). There was also a relation between the low misoprostol exposure (AUC) and the higher rate of induction failure (p-value=0.0052) (Figure 2).

\section{Conclusion}

Weight can influence the bioavailability (AUC) of misoprostol, the higher the weight, the lower the bioavailability and the higher rate of induction failure. 
E1445 - MATERNAL SERUM SCREENING BIOMARKER LEVELS IN PREGNANCIES AFFECTED BY GESTATIONAL DIABETES MELITUS.

Milovanovic Z. ${ }^{1}$, Mihajlovic S. ${ }^{2}$, Karadzov N. ${ }^{1}$, Filimonovic D. ${ }^{3}$

University Hospital "Gak Narodni Front", High Risk Pregnancy Department, Beograd-Serbia ${ }^{1}$ University Hospital “Dragisa Misovic”, Head Of Ob/Gyn Hospital, Beograd- ${ }^{2}$

University Hospital "Gak Narodni Front", Head Of High Risk Pregnancy Department, Beograd $^{3}$

\section{Objective}

To examine the maternal first trimester screening serum biomarkers $\beta$-hCG and PaPP-A as a possible predictor of Gestational Diabetes mellitus

Population: We investigate 210 pregnant women who came for the prenatal control. 30 of them excluded due to an increased risk for chromosomal abnormality, insufficient data or no follow-up. The study included 180 pregnant women, with singleton pregnancies. 106 women who subsequently developed GDM and 74 non-diabetic controls

\section{Methods}

Maternal serum free $\beta$-hCG and PaPP-A were measured at 11,0 to 14,0 weeks of gestation, expressed as multiples of the gestation-specific normal median (MoM). To estimate if pregnant women developed Gestational diabetes mellitus, a standard oGTT was performed between 20,0 to 28,0 gestational weeks.

\section{Results}

Among 180 women included in the study and tested by oGTT 106 were classed as GDM by WHO criteria and 74 were confirmed as unaffected. There were no differences in age, BMI, obstetric and family history, CRL or NT measurements. There was a reduction of $11 \%$ and $13 \%$ in both, maternal median PaPP-A and median free- $\beta$ HCG in GDM groups compared with non-diabetic (control) group, but with no significant statistical difference.

\section{Conclusion}

This study did not find a significant predictive value of isolated biomarkers of the first trimester of pregnancy, $\beta$-hCG and PaPP-a, for early predictions of gestational diabetes mellitus, but it has shown a decrease in value both biomarkers by more than 10 percent in the group of patients who developed GDM. Therefore these independent biomarkers could be a useful and important part of some combined screening for the prediction of the risk of developing gestational diabetes mellitus in this respect should be further investigated. 


\section{Obstetrics - Maternal fetal Doppler: Fetal growth disorders}

\section{E1191 - INTRAUTERINE GROWTH RESTRICTION INCIDENCE RISK FACTORS AND MORBIDITY}

Terzic S. $^{1}$, Maksic H. ${ }^{2}$, Ibrahimovic N. ${ }^{3}$, Vukas E. ${ }^{4}$, Sarajlic M. ${ }^{5}$, Nuhanovic J. ${ }^{6}$

Pediatric Clinic, Ccu Sarajevo, Neonatal Intensive Care Unit, Sarajevo-Bosnia and

Herzegovina $^{1}$

Pediatric Clinic Ccu Sarajevo, Neonatology, Sarajevo-Bosnia and Herzegovina ${ }^{2}$

Pedijatrc Clinic Ccu Sarajevo, Neonatology, Sarajevo-Bosnia and Herzegovina ${ }^{3}$

Pediatric Clinic Ccu Sarajevo, Neonatal Intensive Care Unit, Sarajevo-Bosnia and Herzegovina ${ }^{4}$

Pediatric Clinic, Ccu Sarajevo, Neonatology, Sarajevo-Bosnia and Herzegovina ${ }^{5}$

General Hospital, Neonatology, Sarajevo-Bosnia and Herzegovina ${ }^{6}$

\section{Introduction}

Intrauterine growth restriction (IUGR) is defined by birth weight less then 10th percentile for gestational age, sex, and parity. Factors that may influence growth restriction are: maternal, fetal, uteroplacental and environmental. By the time of occurrence during pregnancy there are different types of IUGR: symmetrical and asymmetrical. If birth weight is under 3rd percentile that is severe form of IUGR, and if within 3rd-10th percentile that is moderate IUGR. Newborns with IUGR suffer from different complications like: perinatal asphyxia, aspiration of amniotic fluid, metabolic disorders, hypothermia, polycythemia, developmental problems and higher incidence of fetal and neonatal mortality.

\section{Objective}

Aim of this paper is to investigate incidence of IUGR, frequency of symmetrical, asymmetrical, severe and moderate form, as well as risk factors and co-morbidity.

\section{Methods}

This is retrospective study, conducted during period of 1st of January 2015 to 30th of June 2016 at the Clinic for Gynaecology and Obstetrician and Neonatology department of Pediatric Clinic, Clinical Center University of Sarajevo. Overall number of term infants with IUGR was 166, divided into two subgroups: 118 who were discharged after delivery (analysed by risk factors for IUGR), and 48 newborns who were hospitalized at the Neonatology department after delivery (additionally analyzed by morbidity).

\section{Results}

Overall incidence of IUGR is $2.9 \%$ (166/5682). Asymmetrical IUGR was significantly more frequent 102/166 (61.4\%) ( $\mathrm{p}=0.005)$. Prenatal factors do not differ in the groups of symmetrical and asymmetrical IUGR. Hypertension, preeclampsia and multiple pregnancies are significantly more frequent in populations of severe and symmetrical IUGR $(\mathrm{p}=0.034$, $\mathrm{p}=0.25)$. That newborns also had significantly longer hospital stay $(\mathrm{p}=0.008 ; \mathrm{p}=0.016)$. Newborns with severe IUGR were more frequently symmetrically growth restricted, and moderately IUGR asymmetrically growth restricted. Perinatal asphyxia and hyperbilirubinemia were dominant co-morbidity in asymmetrical IUGR, and hypoglycaemia and congenital anomalies in symmetrical IUGR.

\section{Conclusion}

Incidence of IUGR in our study was $2.9 \%$. Asymmetrical and moderate IUGR is more frequent. Most frequent risk factors for IUGR are hypertension, preeclampsia and smoking. Perinatal asphyxia and hyperbilirubinemia are dominant co-morbidity in asymmetric IUGR, while hypoglycaemia and congenital anomalies in symmetrical IUGR. 
E1239 - PREDICTIVE VALUE OF EXTREME LOW PAPP A FREE $\beta$ HCG AND EXTREME HIGH MEAN UTERINE ARTERY PULSATILITY INDEX IN THE FIRST TRIMESTER FOR FETAL GROWTH RESTRICTION

Lukic R. ${ }^{1}$, Raicevic T. ${ }^{2}$, Karadzov-Orlic N. ${ }^{1}$, Egic A. ${ }^{1}$, Mikovic Z. ${ }^{1}$

Clinic for Gynecology and Obsteric, Narodni Front, School of Medicine, University of

Belgrade, Department of High-risk Pregnancy, Belgrade-Serbia ${ }^{1}$

Clinic for Gynecology and Obsteric, Narodni Front, School of Medicine, University of Belgrade, Department of High Risk Pregnancy, Belgrade-Serbia ${ }^{2}$

\section{Objective}

In the recent studies it has been shown that the placenta is a field of prenatal screening and diagnosis. Adverse pregnancy outcomes such as preeclampsia (PE), placental abruption (PA), fetal growth restriction (IUGR) and stillbirth could be recognized by prenatal screening. The objective of this study was to predict intrauterine growth restriction (IUGR) using firsttrimester extreme-low pregnancy-associated-plasma-protein-A (PAPP-A), extreme-low freebeta-human-chorionic-gonadotropin (free- $\beta$ hCG)-levels, and extreme-high Pulsatility-index (PI) of uterine arteries, as single and combined predictors for IUGR development.

\section{Methods}

This is a prospective first-trimester study analyzing singleton pregnancies at $11-13+6$ weeks' gestation who underwent routine first-trimester screening at the Department of high risk pregnancy of the Clinic for Gynecology and Obstetrics Narodni front, University of Belgrade, Serbia. First-trimester screening for PAPP-A, free- $\beta \mathrm{hCG}$, and PI was performed in nulliparous, normotensive women with extremely-low PAPP-A (PAPP-A $\leq 0.52 \mathrm{MoM}$ ) and/or extremely-low free- $\beta$ hCG (free- $\beta$ hCG $\leq 0.56 \mathrm{MoM}$ ) and/or extremely-high PI (PI $\geq$ 2.52).

\section{Results}

Of 85 pregnant women included in the final analysis, 14 (16.5 \%) developed IUGR. PAPP-A $\leq 0.52 \mathrm{MoM}$ and $\mathrm{PI} \geq 2.52$, as a single categorical variables, found to be with high predictable values IUGR development $(\mathrm{OR}=3.064,95 \% \mathrm{CI}=0.634-14.810, \mathrm{p}=0.046$ and $\mathrm{OR}=2.129$, $95 \% \mathrm{CI}=0.449-10.713, \mathrm{p}=0.021$, respectively). Furthermore, the ROC-curve identified PAPP-A and PI as continuous variables to be significant predictors of IUGR (AUC $=0.671$, $95 \% \mathrm{CI}=0.521-0.820, \mathrm{p}=0.045$ and $\mathrm{AUC}=0.744,95 \% \mathrm{CI}=0.587-0.902, \mathrm{p}=0.004$, respectively).

\section{Conclusion}

The present study suggests that the first trimester extreme low PAPP-A and increased Doppler-PI levels are single predictors of IUGR. Described model could be used in a routine daily clinical practice in resource limited settings where other parameters are not available for the prediction of IUGR development. 


\section{E1241 - PERINATAL OUTCOME OF PREGNANCIES WITH ABNORMAL} INVASIVE PLACENTA

Theodora M. ${ }^{1}$, Antsaklis P. ${ }^{1}$, Blanas K. ${ }^{1}$, Assimakopoulos G. ${ }^{1}$, Sindos M. ${ }^{1}$, Fasoulakis Z. ${ }^{1}$, Loutradis D. ${ }^{1}$, Daskalakis G.

Medical School, National and Kapodistrian University of Athens, Maternal Fetal Medicine Dpt , 1 Dpt Obstetric Gynecology, Athens-Greece ${ }^{1}$

\section{Objective}

Spontaneous labour in pregnancies with abnormal invasive placenta is relates with obstetrical bleeding and adverse outcome both for the mother and the fetus. Nevertheless, it is debatable if placenta previa and increta/percreta are reasons for fetal compromise. We present our experience on fetal growth patterns in pregnancies with abnormal invasive placenta.

\section{Method}

Forty five cases of pregnancies with abnormal invasion of placenta were included in our study. We followed up the fetuses every 15 days from 28th week up to delivery.

\section{Results}

In thirty four (67\%) cases placenta was found to be previa and in seventeen (37\%) increta/percreta. Gestational age at delivery ranged from 30th weeks up to 38th weeks. Neonatal weight ranged between 9th -67th percentile. There were no cases with fetal compromise during the study. There was no case of emergency delivery due to fetal distress.

\section{Conclusion}

Although abnormal invasive placenta is related to high prevalence of iatrogenic preterm labour and adverse perinatal outcome it seems that this is due to iatrogenic labour and spontaneous labor and bleeding. 


\title{
Obstetrics - Hypertension in Pregnancy
}

\author{
E1027 - PREGNANCY IN RENAL TRANSPLANT RECIPIENTS OUR CENTER \\ OUTCOMES \\ Bayramova T. $^{1}$, Kazımi M. ${ }^{2}$
}

Central Customs Hospital, Obstetric-Gynaecology, Baku-Azerbaijan ${ }^{1}$

Central Customs Hospital, Surgery and Transplantation, Baku-Azerbaijan ${ }^{2}$

\section{Objective}

Renal transplantation (RT) is the only effective treatment for the end-stage renal disease. Although pregnancy after RT is considered to be safe, these patients are difficult to manage for obstetricians. In this study, we aimed to determine maternal and fetal outcomes in pregnancies after RT.

\section{Methods}

We conducted a retrospective review of renal transplant recipients who had received prenatal care and delivered pregnancy at Central Customs Hospital Department of Surgery and Transplantation, Department of Obstetrics and Gynecology January 2010 and December 2018.

\section{Results}

A total of eight pregnancies were identified during the study period. The mean age of the patients at the time of RT was 27.2 \pm 4.3 years (range 17-32 years), and the mean age at conception was 28,3 \pm 6.1 years (range 20-33 years). The mean interval between transplantation and conception was $21.1 \pm 8.2$ months (range 12-36 months). There was no a miscarriage, but stillbirth was observed in one patient. Mean birth week was $36.5 \pm 1.1$ weeks and mean birthweight was $2752 \pm 562 \mathrm{~g}$ (range 2650-3250 g). Three of eight deliveries (37.5\%) occurred before 37 gestational weeks. Preeclampsia was detected in three patients, two pregnancies were complicated by intrauterine growth retardation and one case with stillbirth. Mean postnatal follow-up period was 3.4 \pm 2.6 years (range 1,5-6 years) and all of the babies were healthy. There was no a graft rejection after delivery.

\section{Conclusion}

More favorable pregnancy outcomes can be achieved with a multidisciplinary team and satisfactory counseling is mandatory either preconception and through the pregnancy to reduce maternal-fetal risks. 
E1053 - THE INCIDENCE OF THYROID DYSFUNCTION DURING PREECLAMPSIA IN THE 3RD TRIMESTER OF PREGNANCY AND THE POSTPARTUM

Ait Mouheb T. ${ }^{1}$, Ait Mouheb T. ${ }^{1}$, Benrabah O. ${ }^{2}$, Toudji A. ${ }^{2}$, Djenaoui T. ${ }^{2}$, Djenaoui T. ${ }^{2}$ Faculté De Medecine, Babezzour-Algeria ${ }^{1}$

Faculté De Médecine, Algiers-Algeria ${ }^{2}$

\section{Objective}

To determine the incidence of thyroid dysfunction during preeclampsia in the 3rd trimester of pregnancy and in the postpartum period

Secondarily it's to identify potential risk factors

\section{Methods}

We conducted a longitudinal multicenter cohort study with prospective data. A total of 330 pre-eclamptic women were included between May 2014 and June 2017. A multivariate logistic regression analysis was performed. generalized on confounding factors, estimated adjusted odds ratios (ORs) with 95\% confidence intervals (95\% CI).

\section{Results}

In our study, the incidence of thyroid dysfunction in preeclampsia was 9.7\%: (3\% Isolated hypothyroxinemia, 0.9\% Clinical hypothyroidism, 4.24\% Subclinical hypothyroidism, 0.3\% Clinical hyperthyroidism and $1.21 \%$ Subclinical hyperthyroidism). In univariate analysis, subclinical hypothyroidism was associated with preconceptional obesity. $\mathrm{p}=0.01, \mathrm{RR}=3.5$ CI 95\% [1.3, 9.7], and with iron deficiency anemia $\mathrm{p}=0.001, \mathrm{RR}=6.2695 \% \mathrm{CI}[2.02$, 19.45]. Isolated hypothyroxinemia was significantly related to early gestational age, $34.1 \pm 3$ SA [31.96, 36.24]. In multivariate analysis, superimposed preeclampsia, iron deficiency anemia, and weight gain during pregnancy were associated with an increased risk of dysthyroidism (superimposed preeclampsia: ORa $=4$, CI $[1,3,13]$, iron deficiency anemia iron, ORa = 3.74 IC [1.6e 8.96] and weight gain during pregnancy, ORa = 3 IC [1.34, 6.53] .

\section{Conclusion}

Thyroid function disorders in preeclampsia women have been favored by associated comorbidities. Although we have no information on the therapeutic management of thyroid dysfunction during pregnancy and on Preeclampsia. Our results would suggest that there is a need for better management of thyroid function disorders to improve maternal and fetal prognosis. 
E1068 - HELLP SYNDROME ACCORDING TO A CLINICAL CASE

Gastón B. ${ }^{1}$, Ruiz M. ${ }^{2}$, Bazán M. ${ }^{1}$, Barrenetxea J. ${ }^{1}$, Zabaleta I. ${ }^{1}$, Pedroarena I. ${ }^{1}$, Pérez B. ${ }^{1}$, García S. ${ }^{1}$, Urtasun M. ${ }^{1}$, Abián N. ${ }^{3}$

Complejo Hospitalario De Navarra, Obstetrics and Gynecology Department, PamplonaSpain $^{1}$

Complejo Hospitalario De Navarr, Obstetrics and Gynecology Department Obstetrics and Gynecology Department, Pamplona-Spain ${ }^{2}$

Hospital Reina Sofía, Obstetrics and Gynecology Department, Tudela-Spain ${ }^{3}$

\section{Introduction}

HELLP syndrome is a multisystemic complication during pregnancy characterized by haemolysis, low platelet count and hypertransaminasemia. The prevalence range from 0.1 to $0.8 \%$. Although it is classically defined as the most severe manifestation of preeclampsia, there is current controversia about this association, due to the fact that hypertension or proteinuria is missing in up to $20 \%$ of cases.

\section{Case}

Our patient is a 32 year-old woman, with cesarean section in 36 weeks of previous gestation due to preeclampsia and intrauterine fetal growth restriction. Present gestational follow-up proceed without concerns, until she was attended in Emergency Services at 39+4 weeks relating epigastralgia and subjective reduction of diuresis, in the absence of headache, vomits, or visual disturbances. Mild arterial hypertension was objectified. Complementary studies showed slight hypertransaminasemia and significant proteinuria (300 mg/dl). Against this background, severe preeclampsia was highly suspected: intravenous infusion of magnesium sulphate was started and gestational selective induction of labor was indicated using paracervical prostaglandins under strict fetal and maternal monitoring. After four hours, blood test was repeated revealing low platelet levels and worsen hypertransaminasemia. At this moment, Bishop score continued unfavorable, Consequently, cesarean section was urgently performed owed to HELLP syndrome diagnosis and poor prognosis for vaginal delivery. Uterine atony occurred after fetal and placenta delivery, needing pharmacological measures and B-Lynch suture technique. Immediate puerperium admission in Intensive Care Unite (ICU), requiring antihypertensive treatment, blood transfusion and thomboprophylaxis. Meaningful improvement of analytic parameters was identified the third day, and our patient was discharged two days after with oral hypertensive treatment.

\section{Conclusion}

HELLP syndrome constitutes the most severe demonstration of arterial hypertensive disorders during pregnancy, even though it may be held without arterial hypertension in up to $20 \%$ of cases. Among differential diagnosis, fatty liver syndrome or gestational thrombocytopenia must be taken into account. Patients diagnosed of HELLP syndrome should be treated and closely monitored in ICU. 


\section{E1082 - HELLP SYNDROME CASE REPORT}

$\underline{\text { Ribeiro Ramalho A. }}{ }^{1}$, Fernandes Freitas Maia J. ${ }^{2}$, Ribeiro Simões M. ${ }^{1}$, Ribeiro De Oliveira E. $^{3}$

Maternidade Municipal Mãe Esperança, Ginecology and Obstetricians, Porto Velho-Brazil ${ }^{1}$ Hospital De Base Ary Pinheiro, Resident, Porto Velho-Brazil ${ }^{2}$

Hospital De Base Ary Pinheiro, Ginecology and Obstetricians, Porto Velho-Brazil ${ }^{3}$

\section{Introduction}

HELLP Syndrome is characterized by a triad and its abbreviation stands for: hemolysis $(\mathrm{H}$, hemolytic anemia), liver enzymes (EL) and low platelet count (LP). It occurs with the condition in patients with pre-eclampsia (PE). It is estimated that $8 \%$ of pregnant women with $\mathrm{PE}$ develop the syndrome and it ranges from $0.2 \%$ to $0.6 \%$ of pregnancies.

\section{Objective}

To report a case of a HELLP syndrome, accompanied at the Ary Pinheiro Base Hospital (HBAP) in Porto Velho-RO.

\section{Methods}

The present work uses the reference bibliographical search, to search for information and to report this case on HELLP syndrome.

\section{Case}

Patient P.R.V, 23 years old, IG 34S6D, from Rolim de Moura, admitted to CO-HB on 06/03/16, with severe fronto-occipital headache, coluria and oliguria. She had a convulsive episode on the way. At admission evolved to crisis-convulsive, lethargy and coluria. The physical examination showed a general, lethargic, dyspneic state with a venturi mask $30 \%$, SAT 92\%, tachypneic, hypoxic $(+1 /+4)$, icteric $(+3 /+4)$, afebrile, PA 170x110mmhg AC: BNF, RCR in $2 \mathrm{~T}$ without blows. AR: MV + without adventitious sounds. AFU: 32cm DU-, and vaginal touch: thick, posterior, closed cervix. MMII edema $(+2 /+4)$ homans negative. Laboratory tests: hemoglobin: 9.48, platelets: 75000, bilirubin: T 7.4 D 2.0 I 5.4, AST 1558.8 ALT 663.8 ATSP 66.3 TAP 14.4 INR 1.13. It was carried out dose of attack and maintenance of magnesium sulfate, hydralazine, requested vacancy of ICU, SVD with daily flow of $200 \mathrm{ml}$ oliguria and coluria. Emergency cesarean section was performed, transferred to ICU and prescribed Nifedipine 40mg + Methyldopa 2g / day. Patient needed blood transfusion after procedure with 3 red cells, 2 platelets and 2 plasma. After 4 days in the ICU, she was transferred to a medical clinic ward, where she developed fever and chills, and vancomycin was administered and presented with bulging in FO. She was submitted to USG of abdominal wall that presented anechoic image with hypoechoic content measuring $88 x 46 \mathrm{~mm}$ in the pelvic region, abscess in the pelvic region and bilateral nephritis. She underwent exploratory laparotomy draining supra-aponeurotic hematoma $(50 \times 30) \mathrm{mm}$, moderate amount of blood in the abdominal cavity. In hysterorhaphy and bladder presented organized hematoma of approximately $80 \times 60 \mathrm{~mm}$. After the procedure she was transferred to HB maternity where she presented improvement in the clinical picture. Patient was discharged 23 days after admission. 


\section{E1144 - SUCCESSFUL PREGNANCY AFTER THREE STILLBORNS IN A PATIENT WITH HYPOFIBRINOGENEMIA A RARE HEREDITARY THOMBOPHILIA A CASE REPORT}

Trapani Jr A. ${ }^{1}$, Pivetta Cantarelli M. ${ }^{1}$, Koettker Silveira S. ${ }^{1}$, Henrique May Feuerschuette O. ${ }^{1}$, Souza Da Correggio K. ${ }^{1}$, Gonzaga Del Moral J. ${ }^{1}$, Rossi dos Santos G. ${ }^{1}$, Nascimento Pacheco E. ${ }^{1}$, Portella Martins A. ${ }^{1}$, Toledo Gieburowski J. ${ }^{1}$, Toledo Gieburowski A. ${ }^{1}$ Hu/ufsc/ebserh/ Women's Health Care Unit, Gynecology and Obstetrics, Florianópolis-Brazil ${ }^{1}$

\section{Introduction}

Fibrinogen plays a pivotal role in normal hemostasis by promoting clot formation, platelet aggregation, and fibrinolysis. It has been established that maintenance of hemostatic balance is necessary for successful outcome of pregnancy. Congenital fibrinogen disorders are rare deficiencies and have a prevalence of approximately 1:1,000,000 cases. Hypofibrinogenemia is a subtype of fibrinogen quantitative disorders, in which fibrinogen level is low. These patients are usually asymptomatic, with fibrinogen levels around $1.0 \mathrm{~g} / \mathrm{L}$, sufficient to protect against bleeding.

\section{Case}

The case is about a 21-year-old pregnant woman who had three previous neonatal death due to extreme prematurity and placental insufficiency and, after the diagnosis of a rare thrombophilia, was finally able to deliver a healthy baby. During her first pregnancy she had preeclampsia and placental abruption at gestational age of 23 weeks. The baby was born by vaginal birth and died soon after delivery. After 11 days, she developed an ischemic stroke with good recovery. In her second pregnancy she was treated with unfractionated heparin. Routine ultrasound realized at 23 weeks of gestation demonstrated early fetal growth restriction, absence of amniotic fluid, and altered doppler waveform (reversed end-diastolic flow in umbilical artery and abnormal ductus venosus flow). The baby was delivered by cesarean section and died after 3 days. Placental histologic examination showed intravenous hemorrhage and uteroplacental hematoma. Investigation of thrombophilia revealed a reduced fibrinogen levels $(110 \mathrm{mg} / \mathrm{dL})$ and a prolonged thrombin time $(5,49)$ compatible with the diagnosis of congenital hypofibrinogenemia. One year later, she got pregnant again. Low-dose aspirin prophylaxis (100 mg/day) was prescribed after 17 weeks of gestation. With 25 weeks, the problem repeated. Ultrasound showed early fetal intrauterine growth restriction, oligohydramnios, and altered Doppler waveform (absent enddiastolic flow in umbilical artery and abnormal ductus venosus flow). Two days later, she was submitted to cesarean section due to rapid progression of doppler abnormalities. The newborn survived 5 days. Her fibrinogen level remains stable. Less than one year later, she got pregnant again. It was then initiated unfractionated heparin 10.000UI twice a day and low-dose aspirin. She had no obstetric complications. Considering her bad obstetric history and risk of bleeding, an elective cesarean section was performed at 34 weeks of gestation. The baby weighed 1,850g and received Apgar score of 6/8. He had a good outcome and was discharged home after one week.

\section{Conclusion}

Pregnancy with congenital hypofibrinogenemia is a high-risk condition. Complications include bleeding in early gestation, premature delivery, fetal growth restriction, placental abruption, postpartum hemorrhage, and thrombotic events. In pregnancy, the objective is to achieve serum fibrinogen levels greater than $50 \mathrm{mg} / \mathrm{dL}$ in the first and second trimesters and above $100 \mathrm{mg} / \mathrm{dL}$ in the third trimester. Fibrinogen replacement therapy with fresh frozen plasma (FFP), cryoprecipitate, and fibrinogen concentrate seems to be effective in preventing these complications and reducing the high rate of pregnancy loss. For patients with a history of bleeding and thrombosis, heparin administration can be considered too. 
E1188 - POSTERIOR REVERSIBLE ENCEPHALOPATHY SYNDROME IN SEVERE PREECLAMPSIA

Abian N. ${ }^{1}$, Gaston B. ${ }^{2}$, Ruiz M. ${ }^{2}$, Bazán M. ${ }^{2}$, Barrenetxea J. ${ }^{2}$, Zabaleta I. ${ }^{2}$, Pedroarena I. ${ }^{2}$, Pérez B. ${ }^{2}$, García S. ${ }^{2}$, Urtasun M. ${ }^{2}$

Hospital Reina Sofía, Obstetrics and Gynecology, Tudela-Spain ${ }^{1}$

Complejo Hospitalario De Navarra, Obstetrics and Gynecology, Pamplona-Spain ${ }^{2}$

\section{Introduction}

Posterior reversible encephalopathy syndrome (PRES) is a neurological syndrome associated with different situations, including preeclampsia (PE). This condition may involve alteration of consciousness, visual disturbances and seizures. Symmetric white matter abnormalities, typically in the posterior parietooccipital regions, are often present at magnetic resonance (MRI). We introduce a case of PRES in preeclamptic preterm pregnant patient, that emphasizes the importance of rapid intervention.

\section{Case}

A 32-year-old nulliparous woman at $31+4$ weeks of gestation was transferred from a regional hospital with severe hypertension, headache and mild visual disturbances. Pregnancy previously proceeded uneventfully. PE was suspected at this point, and magnesium sulphate infusion ( $4 \mathrm{~g}$ bolus and then $1 \mathrm{~g} / \mathrm{h}$ by continuous infusion) and fetal pulmonary maturation (6 mg intramuscular betamethasone) were initiated after her admission to the Obstetric Emergency Unit. Fetal and maternal monitoring showed persistent 200/110 mmhg blood pressure, and intravenous labetalol was started. A progressive vision loss until complete blindness occurred in the meantime, and $10 \mathrm{mg}$ oral nifedipine was dispensed. Laboratory exams showed no significant alterations. Cesarean section was performed, after 6 hours of fetal maturation, under regional anesthesia and without complications. The patient was admitted to the Intensive Care Unit. Twenty-four hours after delivery, MRI showed radiological signs indicative of PRES, with cortico-subcortical hyperintense patched injuries in the brain stem of both occipital lobes. Forty-eight hours after delivery, neurological examination was normal, remaining slight blurry vision, and she was transferred to the obstetric unit. She was discharged one week after delivery in good health with antihypertensive therapy (30 mg oral nifedipine). The newborn weighed 1640 grams and presented hyaline membrane disease with satisfactory response to surfactant.

\section{Conclusion}

This is an illustrative case of PRES associated with the acute onset of preeclampsia. It is important to remember that PE can be very unpredictable and manifests in many atypical ways. Differential diagnosis should be excluded as fast as possible, including imaging if neurological manifestations are present. Fast initiation of empirical treatment may be crucial for favorable fetal and maternal outcome. 


\section{E1195 - CAN URINARY NGAL LEVELS BE USED TO CONFIRM PREECLAMPSIA DIAGNOSIS}

Yuksel S. $^{1}$, Ozyurek E. ${ }^{2}$, Gedikbaşı A. ${ }^{3}$

GOP Taksim Training and Research Hospital, Obstetrics and Gynecology, Istanbul-Turkey ${ }^{1}$

Bagcilar Training and Research Hospital, Obstetrics and Gynecology, Istanbul-Turkey ${ }^{2}$

Kanuni Sultan Suleyman Training and Research Hospital, Obstetrics and Gynecology, Istanbul-Turkey ${ }^{3}$

\section{Objective}

Neutrophil gelatinase-associated lipocalin (NGAL) is a $25 \mathrm{kDa}$ protein in the lipocalin family. Preeclampsia is a syndrome consistent of hypertension and proteinuria with inflammation and high anti-angiogenic factor levels. Our purpose in this study is to compare urinary NGAL levels between preeclamptic and healthy pregnancies.

\section{Methods}

A case-control study was performed. Urine samples were collected from women with preeclampsia and normotensive controls matched for age and gestational age. Urinary NGAL concentrations were measured by specific enzyme-linked immunosorbent assay (ELISA).

\section{Results}

123 patients and 58 healthy pregnant women were included. Patients with preeclampsia had significantly higher urinary NGAL concentrations than controls (mean: $387 \mathrm{ng} / \mathrm{ml}$ vs. 188 $\mathrm{ng} / \mathrm{ml}$, respectively; $\mathrm{P}<0.001$ ). Using a cut-off value $252 \mathrm{ng} / \mathrm{ml}$ for urinary NGAL to confirm diagnosis of preeclampsia, sensitivity, and specificity were $92 \%$ and $91 \%$, respectively. The urinary NGAL values of the patients with severe preeclampsia were higher than those in the mild preeclampsia group, (mean: $398 \mathrm{ng} / \mathrm{ml}$ vs. $338 \mathrm{ng} / \mathrm{ml}$, respectively; $\mathrm{P}=0.05$ ).

\section{Conclusion}

Urinary NGAL levels were significantly elevated in women with preeclampsia versus normotensive controls. We also found that urinary NGAL were associated with the severity of preeclampsia. 


\section{E1211 - FOUR CASES OF SEVERE PREECLAMPSIA DEVELOPED BEFORE 22 WEEKS OF GESTATION.}

Kikuchi N. ${ }^{1}$, Miyamoto T. ${ }^{1}$, Ando H. ${ }^{1}$, Kamijo K. ${ }^{2}$, Kanai M. ${ }^{3}$, Shiozawa T. ${ }^{1}$

Shinshu University School of Medicine, Obstetrics and Gynecology, Matsumoto-Japan ${ }^{1}$

Shinshu University School of Health Sciences, Obstetrics and Gynecology, Matsumoto Japan $^{2}$

Shinshu University School of Medicine, Family and Child Nursing, and Midwifery, Matsumoto-Japan ${ }^{3}$

Early-onset preeclampsia is a serious condition of pregnancy with the potential for adverse fetal and maternal outcomes and it is often difficult to manage. Here we report the clinical course of 4 cases of early-onset severe preeclampsia developed before 22 weeks of gestation. The mean maternal age was 36.8 years old (from 34 to 40), and all cases were primigravida and in-vitro fertilization pregnancy. A few days after onset, the blood pressure became severe and two patients had severe proteinuria over $10 \mathrm{~g}$ daily. In addition, severe fetal growth restriction (less than -2.0 S.D.) was observed in all cases. The maternal conditions presented with various organ disorders such as renal dysfunction, respiratory failure due to pleural and peritoneal effusion, and HELLP syndrome. All patients were delivered by cesarean section with indications for maternal organ disorder at between 22 weeks and 26 weeks of gestation. Patient with HELLP syndrome had difficulty in continuing pregnancy, but with regard to severe dyspnea caused by proteinuria or pleural effusion, it was possible to continue the pregnancy for two weeks or more, with careful observation to the maternal and fetal condition. In all cases, blood pressure was normalized and proteinuria was negative after 1 month of delivery.

All infants were SFD infants less than -3.0 S.D., and 2 cases born before 24 weeks gestation were neonatal deaths (birth weight $264 \mathrm{~g}$ and $317 \mathrm{~g}$ ). In two other cases which the gestational period was extended 14 days or more, the infants were born exceeded 24 weeks of gestation and intact survival (birth weight $345 \mathrm{~g}$ and $484 \mathrm{~g}$ ).

Preeclampsia arising before 22 weeks of gestation is difficult to manage because it rapidly develops and becomes severe. It is important to careful observation of the maternal complications and the fetal condition to extend the gestational period as much as possible for the improvement of the newborn's outcomes. In order to further improve the maternal and fetal outcomes, in addition to existing therapies, development of new preventive methods and therapies is required. 


\section{E1216 - PRAVASTATIN IN THE FOLLOW UP AND TREATMENT OF PREECLAMPSIA A CASE REPORT}

Kavak S. $^{1}$, Celik Kavak E. ${ }^{1}$, Keser B. ${ }^{1}$, Batmaz I. ${ }^{1}$, Yener G. ${ }^{1}$

Firat University Faculty of Medicine, Obstetrics and Gynecology, Elazig-Turkey ${ }^{1}$

\section{Objective}

Evaluation of the efficacy of pravastatin in a patient who developed preeclampsia at 32 weeks of gestation

\section{Case}

A patient, whose first pregnancy was terminated by cesarean section at 28 weeks of pregnancy due to severe preeclampsia and who was followed at intensive care unit for 5 days, was admitted to our clinic with the diagnosis of 7 weeks pregnancy just one year after the first pregnancy. The patient was re-evaluated at the 11th weeks of pregnancy by doppler and prophylactic aspirin was started. Regular blood pressure measurements were started at the 20th weeks of pregnancy. The mean pulsatile index of the uterine arteries was 2.8. Results of all doppler evaluations are demonstrated on table 1. Follow up of blood pressure was normal until 28 weeks of gestation. After the $28^{\text {th }}$ weeks, blood pressure increased moderately and it reached to $140 / 90 \mathrm{~mm}$ hg at $29 \mathrm{~W} 6 \mathrm{D}$ of pregnancy. 24 hour urine was collected and a protein level of $420 \mathrm{mg}$ was detected. Upon this, the patient was followed up weekly in the outpatient clinic due to the diagnosis of mild preeclampsia. The patient was hospitalized due to the blood pressure reaching 160/100. Blood examination revealed no pathology. Proteinuria reached to $2278 \mathrm{mg}$. Then $10 \mathrm{mg}$ of pravastatin tablet per day was started. After the administration of pravastatin, blood pressure reached to 140/80 once and afterwards it did not exceed 120/70 and she was followed at outpatient clinic. At 35w3d pregnancy, she was hospitalized due to a blood pressure reaching 160/90 mmhg. There was no abnormality in laboratory results. Due to the persistence of high blood pressures, MGSO4 infusion was started. A female fetus, measuring 2285 grams and $47 \mathrm{~cm}$, was delivered by cesarean section. Her APGAR scores were 9 and 10. Examination of the neonate was normal and was not admitted to the neonatal unit. The mother and newborn were discharged on the third day of delivery without any complication. 


\section{E1270 - ACUTE PULMONARY EDEMA ASSOCIATED TO SEVERE} PREECLAMPSIA IN A 28 WEEK PREGNANCY

Otaño J. ${ }^{1}$, Estefanía A. ${ }^{1}$, Pedroarena I. ${ }^{1}$, Abian N. ${ }^{2}$

Complejo Hospitalario De Navarra, Obstetrics and Gynecology, Pamplona-Spain ${ }^{1}$

Hospital Reina Sofía, Obstetrics and Gynecology, Tudela-Spain ${ }^{2}$

During the last 20 weeks of pregnancy and postpartum, the new onset of hypertension accompanied by proteinuria or significant end-organ dysfunction leads to preeclampsia. This disorder happens in 2,7-8,2\% of women. It is caused by placental and maternal vascular abnormal development, which leads to endothelial damage, being able to cause multisystem organ failure. In this context, pulmonary edema can occur due to the elevation of blood hydrostatic pressure, which is a severity marker of the disease.

\section{Case}

31 year old patient, without medical history of interest

As obstetric-gynecologic history, primary sterility since 2012, partial septate uterus. Pregnancy obtained by in vitro fecundation with embryo transfer in march of 2018. Lack of control of pregnancy between weeks 9 and 24

At 28+4 weeks of pregnancy, the patient was hospitalized because of severe preeclampsia (blood pressure >160/110) without clinical symptoms, for intravenous antihypertensive treatment with labetalol. The analyses where between normal reference ranges: Hemoglobin $11,7 \mathrm{~g} / \mathrm{dl}$, normal platelet account, adequate liver and kidney function. At the day of arrival, fetal lung maturity was begun with the administration of one dose of betamethasone, which was repeated 24 hours before. A perfusion of magnesium sulphate was also initiated. The echography performed the first day showed altered Doppler in uterine vessels, with no fetal blood redistribution.

During the second day, the values of blood pressure got better (150/90) but the analytics worsened, showing a decrease in platelet account and hemoglobin of 9,1g/dl. The patient started to report dyspnea and a chest X-ray was performed, which showed an infiltration in the lower right segment of the lung. That finding, followed by an oxygen saturation of 93\% and a BNP 492pg/ml evidenced a pulmonary edema.

With the pulmonary and neurologic maturation finished and the worsening of the patient, a cesarean section was performed to ensure the well-being of the patient and the fetus, with a suitable result.

The recovery of the mother was satisfactory, with analytic improvement and a decrease on the patient's blood pressure levels, and the newborn evolved properly.

One month after delivery a study of thrombophilia was performed to the patient, with positive findings for the lupus anticoagulant.

Acute pulmonary edema is a multifactorial etiology complication that can curse as a severe complication in preeclampsia. It causes symptoms such as chest pain, decreased oxygen saturation and dyspnea. It is important to make an early diagnose, because as the physiopathology is based on the elevation of blood pressure, it can be predictive of other organ failure. Therefore, it will always be recommended to study any sing related to the respiratory system in this context. 


\section{E1313 - SEVERE PREECLAMPSIA}

AN ADDITIONAL RISK

OF THROMBOEMBOLIC EVENTS

Ait Mouheb T. ${ }^{1}$, Benrabah O. ${ }^{2}$, Guedaoura S. ${ }^{3}$

Eph, Medecine, Algiers-Algeria

Chu, Medecine, Agiers-Algeria ${ }^{2}$

Eph, Médecine, Algiers Algeria ${ }^{3}$

\section{Objective}

Venous thromboembolism (VTE) remains one of the leading causes of maternal mortality and morbidity in developed countries, especially in our country. Pre-eclampsia that complicate up to $7 \%$ of pregnancies, have been shown to be at increased risk of venous thromboembolism. To determine the prevalence of thromboembolic events in severe preeclampsia in patients admitted to our service.

\section{Methods}

We performed a venous doppler ultrasound in all patients with severe pre-eclampsia who experienced symptoms in favor of deep vein thrombosis (DVT) and / or postoperative pulmonary embolism (after Caesarean section) according to their seat, between April 2014 and March 2016.

\section{Results}

The prevalence of thromboembolic events was $0.9 \%$ in patients admitted for severe preeclampsia. Doppler ultrasound has been observed in cases of deep vein thrombosis after cesarean section in pre-eclamptic patients: 3 cases in the right upper limb, 1 cases in the upper left limb, 2 cases in the lower limb left. the 2 cases of segmental pulmonary embolism and one case of massive pulmonary embolism were diagnosed with spiral CT.

\section{Conclusion}

The cases of TVP that we observed in our series could be explained by the severity of preeclampsia in some cases or be related to other obstetric causes. 


\section{E1341 - HOW DIFFICULT COULD BE THE DIAGNOSIS OF ACUTE FATTY LIVER OF PREGNANCY DURING DELIVERY}

Pedroarena I. ' ${ }^{1}$ Zabaleta I. ${ }^{1}$, Abián N. ${ }^{2}$, Barrenetxea J. ${ }^{1}$, Gastón B. ${ }^{1}$, Ruiz M. ${ }^{1}$, Bazán M. ${ }^{1}$, Otaño J. ${ }^{1}$, Estefanía A. ${ }^{1}$

Complejo Hospitalario De Navarra, Obstetrics and Gynecology, Pamplona-Spain ${ }^{1}$

Hospital Reina Sofía, Obstetrics and Gynecology, Tudela-Spain ${ }^{2}$

Acute fatty liver of pregnancy (AFLP) is a rare obstetric emergency characterized by maternal liver dysfunction. It affects 1 in 7000 to 20,000 pregnancies and it usually occurs in the third trimester of pregnancy. Its pathogenesis is not clear but it seems that some defects in fatty acid metabolism are involved. The initial symptoms are nonspecific (nausea, vomiting, and abdominal pain among others) but, if the disease progresses, symptoms of liver failure may appear.

We present a 36-year-old woman, on her fourth pregnancy (one vaginal delivery and two miscarriages), attended at the emergency room for contractions at 39+1 weeks of pregnancy. Low risk pregnancy so far. She refers fatigue and increased edema in the last two weeks but normal blood pressure. During the course of delivery, mild systolic hypertension was observed (140-150 mmHg). An urgent blood test was requested showing elevation of transaminases (AST $535 \mathrm{U} / \mathrm{L}$, ALT $480 \mathrm{U} / \mathrm{L})$, total bilirubin $(3 \mathrm{mg} / \mathrm{dL})$ and creatinine (1.4 $\mathrm{mg} / \mathrm{dL})$, as well as a low platelet count $(124,000 / \mathrm{mL})$, prolonged prothrombin time (16.7 seconds), prolonged INR (1.44) and low fibrinogen rate (354 mg/dL), but no anemia. In view of these results, maternal neuroprotection with magnesium sulfate was performed. Finally, a male fetus was born by normal vaginal delivery. During her stay at the hospital daily blood test controls were carried out, and hemolytic anemia (without schistocytes) was observed. Reticulocytes, LDH and bilirubin were increased and haptoglobin was low. In addition, progressive lengthening of coagulation times, low platelet count $(95.000 / \mathrm{mL})$, hypoglycemia and decreased cholesterol $(129 \mathrm{mg} / \mathrm{dL})$ were evidenced. Leukocyte level, PCR and PCT were increased without symptoms of infection. Due to all these parameters, a precise diagnose of AFLP was made, and therapy with vitamin K initiated. Subsequently, this parameters and blood pressure were progressively normalized, with successful clinical evolution. AFLP can entail to serious fetal and maternal adverse outcome, so an early diagnosis and treatment are crutial. Diagnosis is based on compatible clinic and laboratory criteria, but the differential diagnosis between AFLP, HELLP syndrome and severe preeclampsia may be difficult. Sometimes two of them can appear simultaneously. The definitive treatment consists in the termination of pregnancy, as well as maternal stabilization and recovery of liver function. 
E1412 - PREDICTIVE ROLE OD DEMOGRAPHIC AND CLINICAL RISK FACTORS FOR DEVELOPING PRE ECLAMPSIA AND ITS SEVERITY UNI VARIANT LOGISTIC REGRESSION ANALYSIS

Daneva Markova A. ${ }^{1}$, Dimitrov G. ${ }^{2}$, Tofovski G. ${ }^{3}$, Dzikova E. ${ }^{2}$, Kjaev I. ${ }^{1}$, Hadzi Lega M. ${ }^{1}$, Bina A. ${ }^{4}$

University Clinic of Gynecology and Obstetrics, High Risk Pregnancy, Skopje-Macedonia ${ }^{1}$

University Clinic of Gynecology and Obstetrics, Gyn Oncology, Skopje- ${ }^{2}$

University Clinic of Gynecology and Obstetrics, Infertility, Skopje- ${ }^{3}$

University Clinic of Gynecology and Obstetrics, High Risk Pregnancy, Skopje- ${ }^{4}$

\section{Objective}

To determine whether previously identified risk factors are associated with development of severe form of pre-eclampsia in a heterogeneous cohort of women.

\section{Methods}

Systematic review of data collected form a doctoral case control study among pregnant women coming for consult at our clinic from 2011 till 2014. It consists of investigating the significance of formerly known risk factors in predicting the development of severe form of pre-eclampsia in patients from our country.

\section{Results}

Among the evaluated risk factors our study showed that risk for pre-eclampsia was greater in nullipara women compared with parous. Having a baby at older age is also associated with higher pre-eclampsia risk. The most significant risk factors in predicting not only the development od pre-eclampsia but also its severity is elevated systolic (>160 mm/ Hg) and diastolic (>100 mm/Hg) blood pressure. Factors with non-significant greater probability for developing pre-eclampsia according to our logistic regression analysis are patients with previous pre-eclampsia, smokers, pregnant women with higher BMI and also patients with Diabetes Mellitus ( type I, type II or gestational diabetes).

\section{Conclusion}

The history of elevated systolic and diastolic blood pressure during pregnancy or ever before getting pregnant, pregnancy at older age and null parity can predict the development of preeclampsia and its severity with an increased odds-ratio. Using such variables in regression can help to diagnose pre-eclampsia beforehand and allow timely intervention. 


\section{E1459 - CONTROVERSY IN THE BEHAVIOR OF PATIENTS WITH EISENMENGER'S SYNDROME (ES) AND PREGNANCY \\ Stavinskaia L. ${ }^{1}$, Cojocaru V. ${ }^{2}$, Sarbu Z. ${ }^{1}$, Cospormac V. ${ }^{2}$, Jovmir I. ${ }^{3}$}

State University of Medicine and Pharmacy Nicolae Testemitanu, Department of Obstetrics and Gynecology, Chisinau-Moldova ${ }^{1}$

State University of Medicine and Pharmacy Nicolae Testemitanu, Department of Anesthesiology and Resuscitation, Chisinau-Moldova ${ }^{2}$

Institute of Mother and Child, Department of Anesthesiology and Resuscitation, ChisinauMoldova $^{3}$

Eisenmenger's syndrome is defined as along-standing left-to-right reversal cardiac shunt caused by a congenital heart disease complicated with severs pulmonary arterial hypertension (HTPA). This syndrome in the pregnant women can causes serious complications during the pregnancy, childbirth and post-natal period with increasing level of maternal mortality from $20 \%$ to $65 \%$, and may be attributed to fainting spells, blood clots forming and traveling to distant sites in the veins, hypovolemia, coughing up blood or preeclampsia. We report about clinical case of pregnant " $A$ ” with Eisenmenger's syndrome.

\section{Case}

The patient had been monitored by family doctor since 11-12 weeks of gestation and consequently she was directed to the Republican cardiology center. There woman`s level of HTP was $60 \mathrm{mmHg}$ but she categorically refused to interrupt the pregnancy, after that she was monitored by a multidisciplinary team (cardiologist, heart surgeon and obstetrician) and she got the following treatment with vasodilator and antithrombotic: Seldinophil from $12.5 \mathrm{mg}$ to 25 mg x 3 times; Verospiron 75 mg x2 times; Sol. Clexani 0.2 UA u/s.

Pregnancy had been complicated at 19-20-th week by intrauterine infection (TORCH and Streptococcus beta-hemoliticus) which was cured by cefalosporin and clarithromycin with the main treatment. However at the 23-24 weeks was revealed pregnancy-induced hypertension associated with three-week fetal restriction according to USG date and it led to hospitalization for monitoring and oxygen-therapy. Despite the therapy at the 30th week the patient's general state deteriorated due to acute heart failure (increased fatigue, dyspnea, cough) and the advancing of HTP to $105 \mathrm{mmHg}$, FEof LHV - 45\%, on the background of cavity dilation of left heart ventricular to $182 \mathrm{~mm} 3$.

The patient was connected to the ventilation SPAP mask with FiO2 61\%. According to this treatment, the women`s general state got better: SpO2 increased from $60 \%$ to $94 \%$, HR and the pulse $-80 \mathrm{bpm}, \mathrm{BP}-115 / 80 \mathrm{mmHg}$. However the state of the fetus was aggravated by the circulatory insufficiency of gr. I b with the onset of fetal hypoxia.

Based on the foregoing the pregnancy was completed by emergency cesarean surgery, which was performed under general anesthesia with muscle relaxes and mechanical ventilation. The male fetus was extracted with weight $650 \mathrm{~g}$ according to the scale Apgar 2-4 points. Later 6 hours after the surgery, the artificial ventilation was stopped and the patient was removed under the natural breathing. The postpartum period was held on acute cardiac insufficiency therapy in conditions of pulmonary hypertension (diuretic, seldinophil, nitrate, oxygentherapy in the SPAP mask, morphine analgesia), antiarrhythmic (Verapamil $20 \mathrm{mg} x 2$ times), antithrombotic and uterotonic.

The patient on the 7th day after cesarean surgery was transferred to the specialized cardiology hospital, and then the patient was discharged on the 11th day at home under the supervision of the family doctor. 


\section{Conclusion}

Pregnancy aggravates Eisenmenger syndrome whereas ES itself complicates pregnancy and fetus state. So, it is very serious multisystemic pathology has disastrous maternal and fetal consequences. In this case the pregnancy has to be contraindicated. 


\section{Obstetrics - Maternal nutrition}

\section{E1020 - A CASE STUDY OF ANEMIA IN PREGNANCY WHO UNDERGO ANTE NATALCARE IN OBYGN POLYCLINIC AT CENTRAL CILACAP 1 COMMUNITY HEALTHCENTRE IN PERIOD OF JANUARY TO DECEMBER 2015}

Septina S. ${ }^{1}$, Putri P. H. ${ }^{2}$

Yarsi University, Obgyn, Jakarta-Indonesia

1. Department of Obstetric Gynecology, Faculty of Medicine, YARSI University.

2. Student of Faculty of Medicine, YARSI University

Anemia in pregnancy occurs when there is decreasing of erythrocytes, hemoglobin quantity, or packed red cell volume below normal rate, which is $\mathrm{Hb}<11 \mathrm{~g} / \mathrm{dl}$. This may be caused by several risk factor related with demographic data in ante natal care medical record This study aimed to know about presentation of anemia pregnancy in gravida term in obgyn policlinic Central Cilacap 1 Community Health centre in period of January to December 2015. This is a quantitative descriptive study using ante natal care medical records win Central Cilacap 1 Community Health centre during January to December 2015 (12 months data).

The result of analyzing medical records is majority of pregnant woman had mild anemia (55, $1 \%)$, young adult $(79,8 \%)$, in third trimester $(50,6 \%)$, multigravida $(62,9 \%)$, didn't work (93, 3\%), had normal LILA (74, 2\%), had normal IMT (51, 7\%), and had middle educational status $(53,9 \%)$.

Several risk factor of anemia pregnancy mentioned in previous theory is appropriate with the result of this study. However, there are several results of this study didn't suitable with the previous theory that may be caused by limitation of this study such as short period of study (only one year) and incompleteness of medical records data of ante natal care. 


\section{E1026 - THE RELATIONS BETWEEN MATERNAL BLOOD LIPIDS AND MACROSOMIA \\ Zhou L. ${ }^{1}$, Wang L. ${ }^{1}$, Zhang Y. ${ }^{1}$, Chen X. ${ }^{1}$, Zhang L. ${ }^{1}$, Song S. ${ }^{2}$}

Strategic Support Force Medical Centre of Pla, Department of Gynaecology and Obstetrics, Beijing-China $^{1}$

Center for Special Medicine and Experimental Research, Strategic Support

Force Medical Centre of Pla, Department of Gynaecology and Obstetrics, Beijing-China ${ }^{2}$

\section{Objective}

The aim of this study is to explore the relations between maternal lipids and newborn weight and macrosomia.

\section{Methods}

All subjects in this study were naturally conceived and healthy pregnant women with singleton pregnancies who delivered macrosomia (96 cases) and those who delivered normal weight newborns (140 cases, as control) at full term during the period of January 1st 2017 to December 31st 2017.Heights and weights of women were measured and pregravid body mass index (BMI) was calculated. Lipids were analyzed with fasting blood samples at 36-37 weeks of gestation. Weights and heights of newborns were measured at birth.

\section{Results}

Height, Pre-pregnancy weight and BMI, weight before delivery, weeks of gestation at labor, triglyceride, atherogenic index of plasma, weights and heights of newborns and rates of cesarean section in the group of women having macrosomia newborns were higher significantly than those in control group $\left(\mathrm{t} / \chi^{2}\right.$ value ranged from-4.924 to 6.562 ,all $\left.\mathrm{P}<0.01\right)$. And height, pre-pregnancy weight, pregravid BMI, weight before delivery, weeks of gestation at labor, triglyceride and atherogenic index of plasma were positively correlated with weights of newborns( $\mathrm{r}$ value were $0.183,0.254,0.199,0.280,0.508,0.213$ and 0.238 ,respectively,all $\mathrm{P}<0.01)$.The results of multiple linear regression analysis showed that newborn weight was positively correlated with AIP ( $\beta$ value was $0.42, \mathrm{P}<0.05$ ).

\section{Conclusion}

The blood lipid levels are elevated in pregnant women generally. The increased level of triglyceride in pregnancy may be associated with the risk of macrosomia. In addition, AIP may has certain values in the observing the correlations between blood lipid and macrosomia. 


\section{E1076 - IDIOPATHIC ACUTE PANCREATITIS IN SECOND TRIMESTER OF PREGNANCY CASE REPORT}

Abdalla N. ${ }^{1}$, Piórkowski R. ${ }^{1}$, Stanirowski P. ${ }^{1}$, Bachanek M. ${ }^{1}$, Cendrowski K. ${ }^{1}$, Sawicki W. ${ }^{1}$ Medical University of Warsaw, Chair and Clinic of Obstetrics, Gynecology and Gynecological Oncology, Warsaw-Poland ${ }^{1}$

A 29-year-old patient G4P1A2 was at 32nd week of gestation was referred to a tertiary level hospital because of acute pancreatitis. The patient has hypothyroidism. The patient is non smoker and the course of pregnancy was uneventful. She had one uncomplicated caesarean section five years ago because of fetal macrosomia.

The patient at the day of admission had sudden onset of upper abdominal pain referring to back of the patient and vomiting. She was admitted to primary care level hospital. Laboratory investigations revealed increased serum level of lipase to $1245 \mathrm{U} / \mathrm{l}$, increased WBC count to $14700 / \mu \mathrm{l}$, and $\mathrm{C}$ reactive protein to $11,8 \mathrm{mg} / \mathrm{l}$. The vital signs were in normal range. Ultrasound scan showed edematous pancreas with total longest dimension of $29 \mathrm{~mm}$ with slit like collection of free fluid near the pancreas. There was no evidence of gallstones or biliary lithiasis. Other laboratory investigations were normal. Obstetric physical examination and fetal ultrasound scan did not show abnormalities. Idiopathic mild form of acute pancreatitis according to Atlanta classification was diagnosed. Conservative management was started with intravenous fluid, analgesics (paracetamol and pethidine), and betamethasone was given for fetal lung maturation. The patient was transported to tertiary care level hospital which has both obstetrics and surgical departments. A multidisciplinary team confirmed the diagnosis and continued the conservative method. Subcutaneous heparin was additionally prescribed. A time of admission the serum level of amylase was $713 \mathrm{U} / \mathrm{l}$. Coagulogram was in normal range. Electrolytes results showed only hyponatremia $135 \mathrm{mmol} / \mathrm{l}$. CRP was increased to 21,9 mg/l. Lipid profile was slightly distorted with total cholesterol and triglycerides increased to 257 $\mathrm{mg} / \mathrm{dl}$ and $215 \mathrm{mg} / \mathrm{dl}$, respectively. The next day there was more intense upper abdominal pain, nausea and frequent vomiting. Laboratory investigations revealed higher leukocytosis $17100 / \mu \mathrm{l}$, CRP 117,2 mg/l while serum amylase was $550 \mathrm{U} / \mathrm{l}$. Surgical and gastrological consultations were performed and conservative treatment was continued. The patient's vital signs were in normal range.

Within two days the symptoms of the patient were completely disappeared. The amylase level, leukocyte count dropped to normal value (93 U/l and 11300/ $\mu \mathrm{l}$, respectively). The CRP was $252 \mathrm{mg} / \mathrm{l}$. The patient started to eat without nausea and vomiting. Follow up for the next week in the hospital did not reveal any abnormalities of the vital signs. The patient was in good general condition without symptoms. Abdominal ultrasound features of free fluid disappeared. Throughout hospitalization obstetrical examination did not show abnormalities, cardiotocography was normal.

Exact cause of acute pancreatic in this case was not established. Conservative treatment is the method of choice for mild form of pancreatitis in pregnancy. Multidisciplinary team work is essential for management of such cases. Serial examinations, laboratory investigations and ultrasound are important for monitoring the patient for possible complications. The optimal management should be considered in tertiary level hospital ensuring multidisciplinary care from obstetrics, anesthesia and intensive care, surgery and gastro-enterology units. 
E1110 - PERICONCEPTIONAL MULTIVITAMIN INTAKE IS ASSOCIATED WITH A DECREASED RISK OF CONGENITAL HEART DISEASE - A PROSPECTIVE COHORT STUDY

Wolf $\mathrm{H}^{1}$, Huusom L. ${ }^{1}$, Hegaard H. ${ }^{2}$, Pinborg A. ${ }^{3}$

Hvidovre University Hospital, Gynecology and Obstetrics, Hvidovre-Denmark ${ }^{1}$

Rigshospitalet, Copenhagen University Hospital, Research Unit, Women’s And Children's

Health, Copenhagen-Denmark ${ }^{2}$

Rigshospitalet, Copenhagen University Hospital, Fertility Clinic, Juliane Marie Center for Women, Children and Reproduction, Copenhagen-Denmark ${ }^{3}$

\section{Objective}

Congenital heart disease (CHD) is the most common type of congenital single organ malformation with a prevalence of 8 per 1000 live births. However, little is known about the etiology of CHD, and thus possible modifiable behavioral risk factors. The objective of the study was to assess the association between multivitamin intake and the risk of CHD.

\section{Methods}

The study population comprised of 15,567 women from the Copenhagen Pregnancy Cohort with complete data on multivitamin intake before and during pregnancy who gave birth to live-born singletons from October 2012 to October 2016. Data on multivitamin intake were linked to the Medical Birth Registry to identify the birth outcome. Main outcome measure was CHD defined according to the International Classification of Diseases, 10th revision (codes DQ20-DQ26). Cases of CHD were classified in three subgroups based on the clinical phenotype: 1) septum defects, 2) malformations of great arteries, and 3) other CHD. Multivariate logistic regression analyses were used adjusting for a priori identified potential confounders which included maternal age, pre-pregnancy body mass index, chronic disease, maternal smoking, maternal alcohol consumption, being pregnant after use of assisted reproductive technology and gestational age at birth.

\section{Results}

Of the included women, 31\% had a daily multivitamin intake in the periconceptional period and $54 \%$ in the postconceptional period. The prevalence of CHD in the cohort was $1 \%$. Periconceptional multivitamin intake was associated with a decreased risk of overall CHD (aOR 0.61 (95\% CI 0.37-0.99)), but did not affect the risk of the remaining outcomes. Stratification by body mass index did not alter these findings.

\section{Conclusion}

Few studies have assessed a possible effect of multivitamin intake and CHD. In line with the previous studies, we found that only periconceptional multivitamin intake was associated with a decreased risk of overall CHD. Also, the prevalence of CHD in the cohort corresponded to that previously reported. 


\section{E1172 - THE LEVEL OF ANEMIA DURING PREGNANCY}

Mešalić L. ${ }^{1}$, Begic Z. ${ }^{2}$, Begić M. ${ }^{3}$

Health Centre Tuzla, Women and Pregnant Women Health Protection Service, Tuzla-Bosnia and Herzegovina ${ }^{1}$

Gynecologist Clinic "dr.Begić", Gynecology Clinic "dr.Begić", Kalesija Bosnia and Herzegovina $^{2}$

Medical University of Vienna, Medical University of Vienna, Vienna-Austria ${ }^{3}$

Anemia of the pregnant woman, especially in the early pregnancy, may have a potentially damaging effect on the fetus. In our Service she controlled the pregnancy of 764 pregnant women, of whom 300 pregnant women with anemia, diagnosed with an average age of $26.6 \pm$ 4.7 years, were diagnosed with an average gestation age of $23.9 \pm 9.1$ weeks. They all had clinical signs and laboratory evidence of anemia. The number of erythrocytes (E), hemoglobin $(\mathrm{Hb})$, hematocrit $(\mathrm{Htc})$, and serum iron level $(\mathrm{Fe})$ were analyzed. In the second trimester, the value of $E$ was significantly lower $(p<0.0001)$ than in the first trimester. The mean value $E$ in the third trimester was significantly lower compared to the first $(p<0.0001)$ trimester. In the second trimester, the $\mathrm{Hb}$ value was significantly lower $(\mathrm{p}=0.020)$ compared to the first trimester, while the mean $\mathrm{Hb}$ value in the third trimester was significantly lower than the first trimester $(p<0.0001)$. The mean value of Htc in the third trimester was significantly lower compared to the first trimester ( $\mathrm{p}=0.0008)$. The mean value of Fe in the second trimester was significantly lower compared to the first trimester $(p=0.010)$, while in the third trimester it was significantly lower compared to the first $(\mathrm{p}<0.0001)$ and the second trimester $(\mathrm{p}=$ 0.006). The gestational age in the primigravidas was poorly correlated with the values $E(r=-$ $0.226)$ and $\mathrm{Fe}(\mathrm{r}=-0.276)$. The gestational age in secundigravidas was poorly correlated with the values $\mathrm{E}(\mathrm{r}=-0.366), \mathrm{Hb}(\mathrm{r}=-0.206)$, and $\mathrm{Fe}(\mathrm{r}=-0.280)$. The gestational age at multigravidas was really significant at the border and high correlations for the value $\mathrm{E}(\mathrm{r}=$ $0.688)$, $\mathrm{Hb}(r=-0.695)$, and really significant for Htc $(r=-0.576)$ and easy for Fe $(r=0.206)$.

\section{Conclusion}

With each trimester of pregnancy, anemia becomes more pronounced. In multigravidas with anemia there is a significant association of anemia with gestational age, which should pay special attention to the management of pregnancy, i.e. timely include substitution therapy and advise pregnant women about the quality of the diet. 


\section{Obstetrics - Preterm labor}

E1028 - PREGNANCY OUTCOMES IN ORGAN TRANSPLANT RECIPIENTS A 10 YEAR OUR EXPERIENCE

Bayramova T. ${ }^{1}$, Kazımi M. ${ }^{2}$

Central Customs Hospital, Obstetric-Gynaecology, Baku-Azerbaijan ${ }^{1}$

Central Customs Hospital, Surgery and Transplantation, Baku-Azerbaijan ${ }^{2}$

\section{Objective}

There are an increasing number of reports of pregnancy following liver and renal transplantation, but many questions remain regarding preconception counseling and management of the pregnancy. The aim of this study was to report pregnancy outcomes in women who had undergone liver and renal transplants and to gain insight into these issues.

\section{Methods}

We conducted a retrospective review of liver and renal transplant recipients who had received prenatal care Central Oilworker and Customs Hospitals between January 2010 and December 2018.

Results: 11 consecutive pregnancies in 3 liver and 8 renal transplant recipients were identified during the period. The most common indication for liver transplantation was HBV, for renal transplantation was chronic renal failure. The median age at transplantation was 27 years (range, 17-32). The median age at conception was 28 years (range, 20-33) with a median time between transplantation and conception of 21 months (range, 12-36). A tacrolimus-based immunosuppressive regimen $(\mathrm{n}=11,100 \%)$ was the most common at the time of conception. There were 10 live births (90,9\%), one stillbirth (9,1\%) and no any abortion and miscarriage. Median gestational age at delivery was 37 weeks (range, 34-38), and the median birthweight was 2925 g (range, 2600-3250 g). Pregnancy and maternal complications included preterm deliveries 3 (27,3\%), intrauterine growth restriction $3(27,3 \%)$, pre-eclampsia $3(27,3 \%)$, cesarean delivery $11(100 \%)$, bacterial infection (15\%). We had not any congenital anomalies.

\section{Conclusion}

Pregnancy after liver and renal transplantation can achieve relatively favorable outcomes. Obstetricians should be involved in the contraceptive and fertility counseling of female transplant recipients to prevent unintended pregnancies. 


\section{E1067 - COMPLEX GESTATIONAL MANAGEMENT AN OBSTETRIC} CHALLENGE

Gastón B. ${ }^{1}$, Abián N. ${ }^{2}$, Ruiz J. ${ }^{2}$

Complejo Hospitalario De Navarra, Obstetrics and Gynecology Department, PamplonaSpain $^{1}$

Hospital Reina Sofía, Obstetrics and Gynecology Department, Tudela-Spain ${ }^{2}$

\section{Introduction}

High risk pregnancy not is only associated to at least one circumstance that may pose potential risks for current gestation course, worsening maternal and fetal outcome, but is also related to patients suffering from previous medical diseases that might be negatively affected by gestational situation.

\section{Case}

35 year-old pregnant G4P2C1A0

High risk for neural tube defects in first trimester screening. Prenatal diagnosis scan and amniocentesis dismissed. Hypothyroidism treated with levothyroxine. Symptomatic complete placenta previa requiring short term observational admission. Gestational diabetes controlled by insulin since 29 weeks of gestation. Preterm rupture of membranes at 29+4 weeks, hospitalization for intravenous antibiotic therapy and fetal lung maturation. Admission complicated by Giardia lamblia infection treated by specific antibiotic. Serial ultrasound scans during this time didn't entirely exclude placental acretism at the anterior uterine wall. Threatened preterm labor at $30+6$ week of gestation stopped by intravenous atosiban as a tocolytic therapy. The patient presented general malaise and shivers at $31+1$ weeks, and intravenous antibiotic therapy is restarted as well as intravenous magnesium sulphate for neuroprophylaxis. Blood test parameters and physical examination were indicative of chorioamnionitis, so an emergency cesarean delivery was required. Heavy bleeding during surgery made necessary blood transfusion. Female newborn, 1800 grams. Apgar 7-9, admitted in Intensive Care Unit. Blood culture and placental culture resulted positive for Proteus mirabilis, and intravenous antibiotic therapy was adjusted according to antibiogram. Early postpartum evolution with clinical and analytical improvement. The patient was dismissed at 7th day of puerperium.

\section{Conclusion}

This case report is a clear example of high risk pregnancy. Management is not easy in spite of several diagnostic tools currently available. Gestational follow-up and management of eventual complications based on Multidisciplinary Units may enable work team and decision making in complex patients. 


\section{E1077 - COURSE OF PREGNANCY AND LABOR IN PREGNANT WITH EHLERS DANLOS SYNDROME CASE REPORT}

Abdalla N. ${ }^{1}$, Piórkowski R. ${ }^{1}$, Stanirowski P. ${ }^{1}$, Bachanek M. ${ }^{1}$, Cendrowski K. ${ }^{1}$, Sawicki W. ${ }^{1}$ Medical University of Warsaw, Chair and Clinic of Obstetrics, Gynecology and Gynecological Oncology, Warsaw-Poland ${ }^{1}$

A 22-year-old patient G1P0A0 at 30th weeks of gestation was admitted to the tertiary care level hospital because of gradually increased joint pain, difficulty in moving, breathlessness, multiple faints and unwell feeling. Classic form of Ehlers-Danlos Syndrome was diagnosed clinically in the childhood. She had multiple orthopedic operations of the vertebral column (twice), shoulder joints (twice) and knee joints (twice). The family history was negative for this disease entity. The height of the patient was $160 \mathrm{~cm}$ and the weight was $61 \mathrm{~kg}$ at time of admission.

The pregnancy was planned by the patient and the course of early gestation was uneventful. Fetal ultrasound examination at 13th week and 20th week of gestation did not show any abnormalities. The patient did not have invasive prenatal procedures. She gained about $4 \mathrm{~kg}$ during pregnancy. The patients noticed in the last weeks deterioration in her movement capability and increased joints' pain which was no longer be relieved by paracetamol. Conservative management for the patient was done. A multidisciplinary team of cardiologist, internal medicine physician, anesthesiologist, neurologist, obstetrician, perinatologist and radiologist was involved for monitoring the patient in the third trimester. Obstetrics examination did not show abnormalities. The patient was feeling the fetal movement well, the tococardiography monitoring was reactive. The amniotic fluid index was normal and fetus was growing within normal range. Doppler studies of middle cerebral and umbilical arteries were normal.

Abdominal ultrasound scan, heart echo, Holter ECG investigations did not show abnormalities. Neurological examination did not reveal abnormalities. The trunk of the patient was relatively short. The increase of the abdominal size due to pregnancy causes more pressure on the thorax and elevation of the diaphragm. The patient's mobility was more deteriorated and she was unable to lie flat on her back. Joint pain and dyspnea caused patient's irritation and sleep disturbances. The decision of the multidisciplinary team was to postpone the delivery as much as possible to the moment of most possible fetal maturity. Antenatal steroid was given at 30th week of gestation to stimulate fetal lung maturation. Caesarean section under general anesthesia was done at 34th week of gestation delivering female baby $2310 \mathrm{~g} / 48 \mathrm{~cm}$ with Apgar score of 7-5-8-9. At 1st, 3rd, 5th and 10th minute, respectively. The course of the caesarean section was uneventful. After the delivery the patient's well being was improved and the dyspnea was significantly subsided and the mobility of the patient was improved markedly. Both the mother and baby were discharged to home in good general condition seven weeks after caesarean section.

Management of pregnancy and labor considering rare diseases should be adjusted to individual cases. Multidisciplinary management involving multiple clinical specialties is essential for obtaining the best possible results for patients with rare diseases. 
E1091 - PRETERM BIRTH - RISK FACTORS AND PREDICTORS IN A TERTIARY HOSPITAL IN ROMANIA

Dimienescu O. ${ }^{1}$, Moga M. ${ }^{1}$, Arvatescu C. ${ }^{1}$, Balan A. ${ }^{1}$, Aldea F. ${ }^{1}$, Anastasiu C. ${ }^{1}$

Faculty of Medicine, Transilvania University of Brasov, Department of Medical and

Surgical Specialties, Brasov-Romania ${ }^{1}$

\section{Objective}

The study aimed to observe the prevalence of preterm births and to investigate the obstetrical risk factors in a cohort of pregnant women in a tertiary care hospital.

\section{Methods}

A prospective study was designed and included 1834 pregnant women that presented for different symptoms in at the Clinical Hospital of Obstetrics and Gynecology "Dr.I.A.Sbarcea" Brasov, Romania during two years (2017-2018). Relevant history, obstetric and ultrasonographic examination was performed for all the pregnancies in the 2nd and 3rd trimester.

\section{Results}

From the total number of patients included in our study, $12.37 \%$ delivered before 37 weeks of gestation, $0,43 \%$ presented with abortion ( $<24$ weeks of pregnancy). From the total number of preterm births, we observed that $8.5 \%$ were women with short stature, $4.2 \%$ had the age under 18 years, $10.1 \%$ presented with cervical insufficiency (cervix $<25 \mathrm{~mm}$ ). In $7.2 \%$ of cases, we observed a statistically significant association between preterm birth and oligohydramnios and intrauterine growth restriction.

\section{Conclusions}

The incidence of preterm births was similar in the present cohort with the general prevalence. Cervical insufficiency, oligohydramnios, and IUGR were identified as significant risk factors for preterm births, influencing the neonatal outcome. 


\author{
E1137 - DOMESTIC VIOLENCE IN PREGNANCY RISK FACTORS AND \\ FREQUENCY IN GREECE \\ Antoniou E. ${ }^{1}$, Iatrakis G. ${ }^{1}$ \\ University of West Attica, Midwifery, Nea Smirny-Greece ${ }^{1}$
}

\title{
Objective
}

The objective was to define the context of domestic violence focusing on the prevalence and risk factors in a population of pregnant women in Greece.

\section{Methods}

An anonymous and confidential questionnaire was used to conduct a survey set in the outpatients' clinics of two Public General Hospitals in Athens, in Greece, including five hundred and forty-six women.

\section{Results}

The response rate of the survey was 100\%: four hundred and forty-six questionnaires were given and returned filled in revealing that the prevalence of domestic violence in Greece was 6\% (33 of the participants in the survey) for the period of the survey. More specifically, 3.4\% of the sample of women stated that they had been abused since the beginning of pregnancy. The higher risk of abuse in pregnancy by the partner (61.1\%) was noted for foreign women or Greek women with a foreign partner. This can be explained by the living conditions of immigrant women as well as the customs and traditions from their countries of origin and the fact that they feel isolated and do not know how to seek help. The low educational level is also reported as a main factor. The same applies for work. It was found that women with financial and social independence did not report any abuse whereas housewives and unemployed women or university students face a higher risk $(7.1 \%, 15.4 \%$ and $20 \%$ respectively). The risk of abuse in pregnancy is increased if other factors, such as big age difference, history of abortions and undesired pregnancy (pregnancy which is not desired by the partner increases the percentages of violence by seven times (36.4\% vs. 5.5\%) are also present. Regarding the type of abuse, $2.7 \%$ of the women stated that the abuse was not directed towards a specific part of the body, but it was mainly pushing or verbal abuse. If a part of the body was injured that was mainly the face $(3.1 \%)$ followed by the abdomen (1.3\%), a fact which shows aggressiveness both towards the woman and the fetus. A very important finding is the fact that most of the women stated that they were afraid and also that most of the abused women were not regularly followed up by a physician or did not have all the required tests.

\section{Conclusion}

In the case of domestic abuse, it seems that the period of pregnancy is not a protective factor. Women that are more at risk of experiencing abuse are either immigrant women or women with a foreign partner, unemployed and of low educational level. The absence of a social support network also plays a significant role. The most important period to deal with the domestic violence phenomenon is the prenatal period during which women can be informed about abuse and how to seek help. Furthermore, the health services must be ready to provide support during pregnancy and in the follow-up period. 


\section{E1215 - MANAGING TO BEAT AN OBSTACLE}

Ruiz García M. 1, Bazán Legasa M. ${ }^{1}$, Barrenetxea Arrinda J. ${ }^{1}$, Gastón Moreno B. ${ }^{1}$, Zabaleta Loinaz I. ${ }^{1}$, Abián Franco . $^{2}$, Otaño Ruiz J. ${ }^{1}$, Estefanía Díaz A. ${ }^{1}$, Pedroarena Lecumberri I. ${ }^{1}$

Complejo Hospitalario De Navarra, Gynecologist and Obstetrics, Pamplona-Spain ${ }^{1}$ Hospital Reina Sofía, Gynecologist and Obstetrics, Tudela-Spain ${ }^{2}$

Parkinson's disease is a long-term neurodegenerative disorder that affects predominately dopamine-producing neurons in the substantia nigra, so that it mainly affects the motor system. Symptoms generally develop slowly over years. Young-onset Parkinson's disease occurs in people younger than 40; it affects about 2-10\% of the people with Parkinson's disease. Research suggests that genetics may play more of a role in early or young onset than in people who are diagnosed over the age of 40.

\section{Case}

39-year-old woman with advanced Parkinson's disease for over 10 years, presented at 8 weeks of gestation. She had no other remarkable medical antecedents apart from morbid obesity. As obstetric antecedents, she had a previous forceps vaginal delivery in 2013. When she got to know she was pregnant, she sharply stopped taking most of the drugs she used to for her illness, such as Ropirinole, Rasagiline and Apomorfine, but Levodope. It caused a worsening control of it until her Neurologist could arrange the treatment again. All test made at the beginning of gestation were normal. The only finding was a subclinical hypothyroidism so that she started taking Eutirox.

During all the pregnancy she has been controlled by a multidisciplinary team composed of Neurologist and Obstetrics in order to closely follow her and to adjust her treatment depending on her symptoms. It has been an arduous task since the patient decided to stop taking some of the drugs when she considered.

In the thirtieth week of pregnancy, she underwent a remarkable clinical worsening due to she had notice a slowing-down of its movements and was not even able to move for several hours frequently. The obstetrician committee studied her case and recommended to finished the pregnancy by Caesarean section in 38 to 39 week of pregnancy in case of bad control of the symptoms and not good enough cervical conditions. Otherwise, if a good control of her symptomatology was achieved and the delivery started but its own, it was decided to try a vaginal delivery, remarking she had a previous vaginal delivery a few years ago. At 36 weeks of pregnancy, she consulted at the emergency room for membranes premature ruptured. By that time, although her scare mobility, she has got better control of her symptoms than a few weeks before, so a vaginal delivery intend was proposed. Finally, a female fetus of 3060 was born by normal vaginal delivery, Apgar score of 9/10 and acid-base equilibrium of 7'29. In the immediate postpartum, she asked for inhibiting nursing in order to reinitiate her complete treatment.

\section{Conclusion}

Parkinson's disease is rare in young people; however it can be very debilitation if present. When it occurs in a pregnant woman it is even more difficult to manage. It is also important to have in mind all those drugs they need to intake to achieve a right control of their symptoms. Most of these drugs belong to category C of FDA. It will be essential that we follow the patient closely thanks toto a multidisciplinary team. 


\section{E1217 - FETAL WILMS' TUMOR CASE REPORT}

Kavak S. ${ }^{1}$, Celik Kavak E. ${ }^{1}$, Ozden S. ${ }^{1}$, Batmaz I. ${ }^{1}$, Bulu G. ${ }^{1}$

Firat University Faculty of Medicine, Obstetrics and Gynecology, Elazig-Turkey ${ }^{1}$

\section{Objective}

We herein report a rare fetal renal tumor case that was diagnosed at 33 week 0 days of pregnancy.

\section{Case}

A 22-year-old G1POAOCO patient was referred to our clinic with the diagnosis of biparietal diameter-abdominal circumference disproportion and a fetal weight gain of $1000 \mathrm{mg}$ in 3 weeks. She had no significant medical or family history. Ultrasonographic examination revealed a female fetus whose measurements except the abdominal circumference were consistent with the gestational age. Abdominal circumference was $377 \mathrm{~mm}$ and it was consistent with 42 weeks. Amniotic fluid was measured as $96 \mathrm{~mm}$ in the deepest pocket. A mass measuring $72 \times 90 \mathrm{~mm}$, that pushes the descending aorta, vena cava inferior and the intestines to left was, determined on the right side of the abdomen (Figures 1 and 2). Coronal examination revealed that the mass was completely arising from the right kidney (Figure 3). Corticosteroids were given to induce pulmonary maturation. At 33w4d, spontaneous contractions started and she underwent cesarean section. Apgar scores were 8 and 6 respectively. On the third day, she underwent surgery and complete tumor excision was performed without any complication. The frozen examination of the mass was reported as Wilms' tumor. Case was referred to pediatric oncology department.

\section{Conclusion}

Congenital Wilms tumors are seen very rare. Polyhydramnios is frequently associated with renal tumors due to the compression of major infradiaphragmatic vessels. Rapid growth of the tumor mostly needs rapid intervention. 


\section{E1256 - COMPARISON OF MISOPROSTOL VERSUS DINOPROSTONE FOR INDUCTION OF LABOR AT TERM}

Morales F. ${ }^{1}$, Galarce V. ${ }^{2}$, Salvo I. ${ }^{1}$, Guerra M. $^{3}$

Universidad Finis Terrae, Ginecoobstetricia, Santiago-Chile ${ }^{1}$

Hospital El Carmen, Ginecoobstetricia, Santiago-Chile ${ }^{2}$

Universidad De Santiago De Chile, Ginecoobstetricia, Santiago-Chile ${ }^{3}$

\section{Objective}

To compare the efficacy and safety of vaginal misoprostol and removable dinoprostone vaginal delivery system (Propess) for the induction of labor in pregnant women between 37 and 41 weeks of gestation

\section{Methods}

In this retrospective observational study, 200 patients with a single pregnancy, 37 - 41 weeks, cephalic presentation, Bishop score $<6$, admitted for labor induction, were evaluated from October 2016 to September 2017. Misoprostol Group (100 patients) received 50 micrograms vaginally every 6 hours to a maximum of 4 doses and Dinoprostone Group (100 patients) received dinoprostone $10 \mathrm{mg}$ vaginally for once up to 24 hours. Women were monitored by external CGT through labor induction and examined vaginally every 6 hours to assess the progress of induction progress. The primary outcome was percentage of women who reached the active phase. Secondary outcomes included vaginal delivery, emergency C-Section, maternal complications and neonatal outcomes.

\section{Results}

There were no significant differences between the two groups in terms of Maternal age, Gestational age, , BMI, and Parity. There were no significant differences in our primary outcome 74\% misoprostol group versus $72 \%$ dinoprostone group (p 0.803 Pearson 's chisquared test). No significant difference was observed for women delivering vaginally $65 \%$ misoprostol versus 61\% dinoprostone (p 0.478 Pearson's chi-squared test), cesarean deliveries, hyperstimulation syndrome, apgar score and neonatal outcomes

\section{Conclusion}

Misoprostol is as efficacious and safe as Dinoprostone for labor induction 


\section{E1290 - DEALING WITH A SHORT CERVIX IN THE SECOND TRIMESTER OF PREGNANCY}

Psarris A. ${ }^{1}$, Sindos M. ${ }^{1}$, Theodora M. ${ }^{1}$, Antsaklis P. ${ }^{1}$, Anastasakis E. ${ }^{2}$, Loutradis D. ${ }^{1}$, Daskalakis G. ${ }^{1}$

National and Kapodistrian University of Athens, Alexandra Hospital, 1st Department of

Obstetrics and Gynecology, Athens-Greece ${ }^{1}$

Naval Hospital, Department of Obstetrics and Gynecology, Athens-Greece ${ }^{2}$

\section{Objective}

Our objective was to evaluate the results of the implementation of different management protocols in women with a short cervix in the second trimester of pregnancy.

\section{Methods}

The study included all pregnant women from our outpatient maternity department with a cervical length of $\leq 25 \mathrm{~mm}$ during a seven-year period. Women with a short cervix were offered two management options. The first included the placement of a vaginal pessary in combination with $200 \mathrm{mg}$ vaginal progesterone every night from diagnosis to 37 weeks. The second was the administration of either $200 \mathrm{mg}$ of micronized progesterone, or $80 \mathrm{mg}$ of progesterone gel every night and close follow-up with serial transvaginal scans to assess the cervical length. When shortening of the cervix less than $15 \mathrm{~mm}$ until 26 weeks was detected, cervical cerclage was offered, as progesterone was no longer able to inhibit cervical changes. A modified McDonald technique was implemented under regional anesthesia. Vaginal progesterone administration was continued after the cervical cerclage. Exclusion criteria were multiple gestation, a maternal age less than 18 years, major fetal anomalies, placenta previa, active vaginal bleeding, presence of a cerclage in situ and symptoms of preterm labor. Women with ballooning membranes (beyond the external os) were also excluded from the pessary placement. The primary study outcomes were preterm delivery rate (before 34 and 32 weeks of gestation) and pregnancy prolongation. The secondary variables included mean gestational age at birth and birthweight.

\section{Results}

Cervical length $\leq 25 \mathrm{~mm}$ was observed in 245 women. One was excluded as she declined treatment with either cerclage or a pessary despite the fact that she had a very short cervix at the initial evaluation $(10 \mathrm{~mm})$. Pessary placement in combination with vaginal progesterone was offered to 144 out of 245 women. As for the remaining 100 women, 25 of them were managed with cervical cerclage because of a cervical length $<15 \mathrm{~mm}$ at the initial evaluation, while 75 received vaginal progesterone. Of the latter, 37 women had a cervix $<15 \mathrm{~mm}$ during the follow-up visits and they were also managed with cerclage, thus only 38 women remained to the only progesterone group. The preterm delivery rate before 34 and 32 weeks was 10.4 and $6.3 \%$ respectively for the pessary group, 20 and 12\% for the cerclage group, 13.5 and 8.1\% for the progesterone +cerclage group, and 10.5 and $5.3 \%$ for the progesterone only group. The mean latency period was $15,14.4,15.3$ and 16 weeks for the pessary, the cerclage, the progesterone+cerclage and the progesterone only group, respectively. These results were not significantly different. No difference was also observed among the 4 groups regarding the secondary outcomes. Table 2 presents the pregnancy outcome in the 4 groups.

\section{Conclusion}

Applying 2 different management protocols with 4 different interventions in pregnant women with a second trimester short cervix led to similar perinatal outcomes regardless of the intervention that was selected. 


\section{E1294 - PLACENTAL ALPHA MICROGLOBULIN 1 TO PREDICT SPONTANEOUS PRETERM BIRTH IN SYMPTOMATIC WOMAN}

Morales F. ${ }^{1}$, Galarce V. ${ }^{1}$, Guerra M. ${ }^{2}$

Universidad Finis Terra, Ginecologia Y Obstetricia, Santiago-Chile ${ }^{1}$ Universidad De

Santiago De Chile, Ginecologia Y Obstetricia, Santiago-Chile ${ }^{2}$

\section{Objective}

The objetive of this study was to determine the effectiveness of Placental Alpha Microglobulin-1 (PAMG-1) to predict preterm birth at 7 and 14 days from the time of assessment.

\section{Methods}

We conduced a prospective observational study, between April 2018 to April 2019, the study population consist of 60 consecutive pregnant women with symptoms of preterm labor between $24+0$ and $33+6$ weeks of gestation with singleton or twin pregnancies, cervical length between 20-30 mm and intact membranes. PAMG-1 was realized at the time of admission according with the provider instructions and the test to delivery interval was calculated. Patients with PAMG-1 positive test were managed at our High-Risk Maternity Unit according to our preterm birth protocol.

\section{Results}

60 patients were analyzed. The overall rate of preterm birth was $8 \%$ within seven days and $12 \%$ within fourteen days of testing. 18\% (11/60) had PAMG-1 positive and 82\% (49/60) had PAMG-1 negative. The positive predictive value and negative predictive value for spontaneous preterm delivery within 7 days was 18\% (2/9)PPV and 98\% (1/45) 14 days was 18\% PPV (2/9) and 94\% NPV(3/45) respectively.

\section{Conclusion}

A negative PAGM-1 test in pregnant women presenting with symptoms of preterm labor and cervical length between 20-30 mm indicated that spontaneous preterm birth within 14 days is very unlikely. Our outcomes are in accordance with the results published 7. PAMG-1 is easy to use and his excellent performance to rule out preterm birth will result in less hospitalization, use of tocolysis, corticosteroids and in general a saving of economic and human resources. 


\section{E1297 - THE ASSOCIATION OF ANTENATAL CORTICOSTEROID ADMINISTRATION AND FETAL OUTCOME IN INFANTS WITH LOW BIRTH WEIGHT}

$\underline{\text { Tsikouras } P .} .^{1}$, Deuteraiou D. ${ }^{1}$, Anthoulaki X. ${ }^{1}$, Bothou A. ${ }^{2}$, Chalkidou A. ${ }^{1}$, Gaitatzi F. ${ }^{1}$, Babageorgaka E. ${ }^{1}$, Tsirkas I. ${ }^{1}$, Michalopoulos S. ${ }^{1}$, Koukoulomati A. ${ }^{1}$, Soilemtzidis M. ${ }^{1}$, Zervoudis S. ${ }^{3}$, Galazios G. ${ }^{1}$

Democritus University of Thrace, Obstetrics and Gynecology, Alexandroupolis-Greece ${ }^{1}$

Democritus University of Thrace, Obstetrics and Gynecology, Alexandroupoolis-Greece ${ }^{2}$

Rhea Hospital, Obstetrics and Gynecology, Athens-Greece ${ }^{3}$

\section{Objective}

Preterm labour is associated with high perinatal morbidity and mortality. The goal of this study was to evaluate and compare the obstetric outcome in infants with birth weight lower than 1.500 gr.

\section{Methods}

In this retrospective study (January 1997- December 2017) 280 women in third trimester of uncomplicated singleton pregnancy were enrolled. Group 1 is consisting from 120 cases which infants born after two doses of $12 \mathrm{mg}$ betamethazone 24 hours apart. Group 2 includes 100 cases where infants born after only one dose of $12 \mathrm{mg}$ betamethazone due to progress of labour. Finally, in Group 3, 60 infants born without administration of antenatal steroids also due to progress of labour are comprised. All participants were informed about the use of corticosteroids and gave their written consent. Cases diagnosed with premature membrane rupture or premature contractions are excluded. Apgar score at one and five minutes, the birth weight among newborns, the assessment of the severity of neonatal respiratory morbidity depending on the admission in NICU, the duration of hospitalization, and complications in the NICU were evaluated.

\section{Results}

The complication incidence was significantly higher in the Group 3. 32,5\% were born with acute respiratory disorders, 30\% with transient tachypnea, 10\% severe RDS (respiratory disease syndrome), $1 \%$ were dead and $8 \%$ had intraventicular hemorrhage or necrotizing enterocolitis. In subgroups 1 and 2 the mobidity and mortality were significantly lower. In the Group 1 based on full corticosteroids administration and in association to increasing pregnancy age more than 28 weeks very satisfactory perinatal outcomes were noticed.

\section{Conclusion}

The complete treatment of antenatal steroids is associated to perinatal outcome improvement in full term infants as well as in preterm infants with low born weight. 


\section{E1309 - ESOPHAGUS ATRESIA FETUS OF A PREGNANT ATTENDED IN A POLYCLINIC OF THE WESTERN AMAZON CASE REPORT}

Ribeiro Simões M. ${ }^{1}$, Santos Marçal M. ${ }^{2}$, Simões Lamego L. ${ }^{3}$, Simões Lamego G. ${ }^{4}$, Ribeiro Freitas P. ${ }^{5}$, Gloria Da Silveira E. ${ }^{6}$, Pandolfi Freitas T.

Faculdades Integradas Aparicio Carvalho, Medicina, Porto Velho-ro-Brazil ${ }^{1}$

Hospital Samar, Ginecologia E Obstetricia, Porto Velho-ro-Brazil ${ }^{2}$

Hospital Getúlio Vargas, Urgência E Emergência, Tramandai-rs-Brazil ${ }^{3}$

Gso Medicina Ocupacional, Medicina Do Trabalho, Esteio-rs-Brazil ${ }^{4}$

Clinica Maria Do Carmo, Ultrassonografia, Porto Velho-ro-Brazil ${ }^{5}$

Universidade Federal De Rondônia, Geografia, Porto Velho-ro-Brazil $^{6}$

\section{Introduction}

Esophageal atresia is a congenital anomaly, where there is narrowing or complete obstruction of the esophageal lumen. It has a higher incidence in twins, an increase in maternal age and primiparity. It occurs with an incidence of one case among 3,000 live births, more common in whites and males. On prenatal ultrasound, esophageal atresia may be suggested by the presence of polyhydramnios, decreased intra-luminal fluid in the fetal gut, no visualization of the stomach, and a distal proximal pouch of the atretic esophagus may be visible.

\section{Objective}

To report a case of esophageal atresia in the fetus of a pregnant woman attending a Policlinic in the Western Amazon and review literature of greater relevance on the subject. This work uses a bibliographical research reference, in order to obtain information on the subject and to report the case of esophageal atresia in the fetus of a pregnant woman attended to in a Polyclinic of the Western Amazon.

\section{Case}

M.I.O., 33 years old, a 35 week pregnant woman, is diagnosed with esophageal atresia during routine exams. Result of US with gestational age of 32 weeks and 2 days, cephalic fetus, weight 3131gr, normal amniotic fluid, heterogeneous placenta grade I, with absence of gastric bubble and percentile weight less than 03, with restriction of intrauterine growth . Referred to prenatal high-risk for follow-up. She came in preterm labor with 35s6d, vaginal delivery, live fetus, crying at birth, APGAR 6/8, weight 1350gr, was referred to a neonatal intensive care unit.

\section{Conclusion}

It is very important the prenatal follow-up with the imaging tests to detect the fetal anomaly and establishment of the treatment at birth. The early diagnosis of atresia in prenatal ultrasound scans will serve as a valuable aid in the immediate postnatal approach, maintaining low mortality rates and perinatal morbidity 
E1312 - HOW THE INTERPRETATION OF CARDIOTOCOGRAPHIC TRACE CANNOT PREDICT ALWAYS PERINATAL OUTCOME

Zabaleta I. ${ }^{1}$, Pedroarena I. ${ }^{1}$, Perez B. ${ }^{1}$, García S. ${ }^{1}$, Urtasun M. ${ }^{2}$, Abian ${ }^{2} .^{1}$, Gastón B. ${ }^{1}$, Ruiz M. ${ }^{1}$, Bazán M. ${ }^{1}$, Barrenetxea J. ${ }^{1}$

Complejo Hospitalario De Navarra, Ginecology, Pamplona-Spain ${ }^{1}$

Compolejo Hospitalario De Navarra, Ginecology, Pamplona-Spain ${ }^{2}$

\section{Introduction}

Fetal health is evaluated, in part, by assessment of fetal heart rate patterns. The primary objective of intrapartum fetal heart rate is to assess the adequacy of fetal oxygenation and presence of fetal metabolic acidemia during labor so that timely intervention can be undertake to reduce the neonatal morbi-mortality. Despite a proper interpretation of the cardiotocographic traces, sometimes we can't prevent a fatal outcome. The interpretations of cardiotocographic trace in premature fetus it's more difficult and they have complications of premature fetus so the fatal outcomes can be more frequent.

\section{Case}

Pregnant of 32 weeks and 4 days of gestational age

She started with work of labor and short cervix length. After 189 minutes of labor, with a satisfactory cardiotocographic trace, she gave birth a newborn with cardiac arrest. The Apgar score was 1,2 and 5 at first, fifth and tenth minute consecutively. The umbilical cord $\mathrm{pH}$ analysis score was 6.54 in arterial blood. The newborn was hospitalized at neonatal intensive area but finally he died at third day of life.

\section{Conclusion}

Unless we do a correct cardiotocographic control and interpretation, sometimes the fetus has some pathologies, and more complications on premature fetus, that we can't diagnose them by sonography examination, and we can have fatal perinatal outcomes without a previous suspicion. 
E1346 - PROGRESSION OF THE SECOND FETUS TO NEAR TERM THROUGH PERFORMING THE EMERGENCY CERVICAL CERCLAGE BECAUSE OF ABORTION OF ONE FETUS AT 17TH WEEKS OF GESTATION

Yalinkaya A. ${ }^{1}$, Elci E. ${ }^{1}$, Afsin Y. ${ }^{2}$

Dicle University Medicine Faculty, Obstetrics and Gynecology, Diyarbakir-Turkey ${ }^{1}$

Private Batman Life Hospital, Obstetrics and Gynecology, Batman-Turkey ${ }^{2}$

A 22 years old patient (G1P0) referred to us due to threatened abortion. She had been pregnant by IVF because of previous abdominopelvic tuberculosis and surgery. There were lower and upper midline incision scars on the abdominal wall. On ultrasound examination; dichorionic-diamniotic twin pregnancy was detected. There were not found any anomaly for both fetuses. The first fetus was in breech presentation, the amniotic membrane was ruptured. Lower half of fetal body was seen in the cervical canal and vagina. The second fetus was seen in uterine cavity with normal amniotic fluid.

After examination the patient transferred to operation room. Under sterile conditions and mask anesthesia, the first fetus extracted, then the umbilical cord, amniotic membranes and placenta pulled down with ovarian clamps and extracted. The emergency cervical cerclage was performed. The patient was treated against infections about ten days. Then, the patient was followed up until 37th weeks of gestation. Towards the end of pregnancy mild preeclampsia developed and therefore Alphamethyldopa was given. At the 37 weeks of gestation due to uterine contraction cesarean section was performed, $2300 \mathrm{~g}$ healthy baby was delivered without any undesirable situation.

We think that, if a pregnancy is overly desirable by a couple, we can help in this way. 


\section{E1358 - THE EFFECTS OF EMERGENCY CERVICAL CERCLAGE AFTER AMNIOREDUCTION ON PROGNOSIS OF PREGNANCY}

Yalinkaya A. ${ }^{1}$, Elci E. ${ }^{1}$, Bozbay N. ${ }^{1}$

Dicle University Medical Faculty, Obstetrics and Gynecology, Diyarbakir-Turkey ${ }^{1}$

\section{Objective}

The aim of this study is to investigate the effects of emergency cervical cerclage (ECC) after amnioreduction in patients with complete cervical patency and amniotic sac prolapse to the vagina.

\section{Methods}

This study was designed retrospectively for ECC cases out of 68 patients between April 2014 and May 2019. The patients data were obtained from hospital files and perinatologist ultrasonography records. In addition, all patients were phoned to find out about their latest status. Only 27 patients who underwent ECC by a single surgeon and whose all information was obtained were included in the study. All patients underwent fetal and cervical examinations by only an experienced perinatologist. All patients were informed and informed consent was obtained Transabdominal amnioreduction was performed in all patients before cerclage, and ECC was performed immediately under general or local anesthesia in operating room conditions. Mersilene tape suture was used for cerclage and supported with prolene suture. All patients were treated with prophylactic antibiotic and tocolytic treatment. Patients were mobilized from the first postoperative day, and discharged when their treatment were finished.

\section{Results}

The mean age of patients who underwent ECC was $27.03 \pm 4.83$ (18-38), gravida was $2.37 \pm$ 1.64 (1-7), parity was $0.48 \pm 2.12(0-4)$, abortion was $1.0 \pm 1.30(0-4)$ and living child was $0.22 \pm 0.64$ (0-3). It was found that $16(59.25 \%)$ of the patients were spontaneous, 7 (25.92\%) were pregnant by IVF, 4 (14.81\%) were induced by ovulation induction and 8 (29.62\%) were twin pregnancies. The mean amount of amnioreduction was $248.82 \pm 128.34$ (110-420) ml. The mean gestational age of the patients who underwent ECC was $20.74 \pm 2.24$ (16-25). The mean gestational age at delivery was found as $29.5 \pm 6.76$ (16-39) and patient's pregnancy was continued. Mean birth week was found as $30.16 \pm 7.53$ (16-39) weeks in all single pregnancies and $28.0 \pm 4.65$ (22-34) weeks in twin pregnancies. Survival of 17 (62.96\%) pregnancies and fetuses, 4 of them twins and 1 ongoing pregnancy, were achieved in all cases that underwent ECC. The mean weight of 20 live fetuses born was $2120 \pm 565.68$ and the total weight of 34 newborns was $1454 \pm 998$ (200-3250), in single cases $1718 \pm 1155$ (200$3250)$ and in twin cases $1156 \pm 708$ (450-2400). Cerebral palsy developed in one of the twin partners and the other babies were found healthy.

\section{Conclusion}

Pregnancy with cervical patency and amniotic sac prolapse to the vagina in the pre-viability period usually results in loss. In our study, ECC after amnioreduction was performed in cases with full cervical opening and amniotic sac prolapse to vagina. Our success rate with ECC was $68.42 \%$ in single pregnancies, $50 \%$ in twin pregnancies, and $62.96 \%$ in all pregnancies. If ECC is performed by experienced surgeons, they can survive a significant proportion of the fetuses that will be lost. Therefore, we recommend that, after giving detailed information to patients about their situation and obtaining their consent the ECC may be perform by experienced surgeons. 
E1376 - GIANT UTERINE MYOMA AND PREGNANCY

Guerra M. ${ }^{1}$, Nuñez A. ${ }^{1}$, Haye M. ${ }^{1}$, Farias L. ${ }^{1}$, Gutierrez J. ${ }^{1}$

Hospital Clínico San José, Obstetricia Y Ginecología, Santiago-Chile ${ }^{1}$

\section{Introduction}

Uterine myomatosis is the most frequent benign tumor of the female genital tract, increasing progressively with age and reaching a peak at 40s. Because of this, they may be present when a pregnancy is conceived. Most of them do not experience changes during pregnancy, although $1 / 3$ tends to grow mainly in the first trimester. The diagnosis and follow-up is basically clinical and echographic. The surgical treatment of these is reserved only for very specific cases such as: red degeneration that does not yield to the use of analgesia, torsion of this when pedicled, or obstruction of neighboring organs.

\section{Case}

A 36-year-old African-American patient, enrolled in a 14-week pregnancy, was admitted to the San José Hospital, Feto-maternal Unit, due to abdominopelvic pain that did not yield to usual analgesia, associated with urinary retention. Ultrasound and MIR inform intrauterine gestation according to gestational age; uterus with multiple fibroids, the largest being located on the anterior wall and fundus, subserous, pedunculated, measuring $24 \mathrm{~cm}$ in its maximum diameter and bilateral hydroureteronephrosis.

An exploratory laparotomy was performed, showing a large pedunculated myoma measuring $40 \mathrm{~cm}$ in diameter, at the uterine fundus. We proceed to resect myoma accessing $2 / 3$ of the uterine wall without reaching cavity. Myoma weight 3960 grs. The uterus is sutured in double plane, with vicryl and prolene, hemostasis and abdominal wall closure by planes. Biopsy reports leiomyoma with focal involution signs.

Patient evolves with a favorable post-surgical being discharged at the 4th day. Subsequently, it is controlled by the Feto-maternal Unit and a cesarean is performed at 37 weeks, by PPROM, giving a male of 3340 grs in good condition.

\section{Conclusion}

Although it is true, uterine myomatosis can be found associated with pregnancy, it is uncommon for it to be complicated $(<10 \%)$.

Myomectomy is a procedure rarely described in pregnancy, this is because of its high risk of bleeding and the probability of hysterectomy. The most frequent indication for a myomectomy is the pain that does not subside with analgesia, as happened with our patient. The moment of surgery is mentioned in the literature as ideal between 16 and 20 weeks, since it is associated with a lower risk of adverse perinatal outcomes. Finally, the cesarean was performed because there would be a contraindication for vaginal delivery due to risk of uterine rupture. 


\section{E1421 - WEGENER GRANULOMATOSIS AND IVF}

Dokuzeylul Gungor N. ${ }^{1}$

BAU Goztepe Medicalpark, IVF, Istanbul-Turkey ${ }^{1}$

Wegener's granulomatosis is a multisystem life threatening vasculitis which poses particular concerns when it involves a pregnant patient. We present a patient with Wegener's granulomatosis whose pregnancy was achieved by IVF treatment. Granulomatosis with polyangiitis (GPA) is an autoimmune disease which has a variable clinical presentation and usually progresses from a localized to a generalized form over the course of weeks to years. Pregnancy in patients with GPA is burdened with the risk of possible complications and increased mortality and the conception should be delayed until remission of the disease. A 35year-old multigravida with EGPA was referred to our hospital because of 12 years -secondary infertility due to severe male factor. She had negative ANA (antinuclear antibodies), dsDNA (double-stranded DNA), ENA (extractable nuclear antigens-RNP, Sm, Ro, La) antibodies. ANCA (using indirect immunofluorescence test-IFT), and her antiphospholipid antibodies were also negative. We used antagonist protocol and obtained 5 oocytes. On planned frozen cycle, we gave 2 day 5 embryos with high quality. She was treated successfully by steroids during pregnancy. Prednisolone treatment was maintained until labor. Routine visits were performed, and fetal growth was normal, and screenings for congenital anomalies and diabetes revealed no abnormal results. With a multidisciplinary approach, she had a successful caesarean section of a healthy twin babies at 31th weeks of gestation because of early rupture of membranes. Her pregnancy and postoperative course was uneventful and she left the hospital in 3 days. The best time to plan conception is a minimum of six months after entering remission. It is beneficial to stop potentially toxic immunosuppressants (if it is possible) and the woman and child should be closely monitored by obstetricians and rheumatologists. There are almost 40 cases of WG in pregnancy reported in the literature. Owing to this rarity, the management is individualized and the pregnancy outcome is variable. 


\section{E1468 - EFFECTS OF CERVICAL CERCLAGE ON PREGNANCY OUTCOMES ACCORDING TO DEGREE OF CERVICAL PATENCY}

Yalinkaya A. ${ }^{1}$, Elci E. ${ }^{1}$, Bozbay N. ${ }^{1}$

Dicle University Medical Faculty, Obstetrics and Gynecology, Diyarbakir-Turkey ${ }^{1}$

\section{Objective}

The aim of this study is to investigate the effects of cerclage on pregnancy prognosis due to complete cervical patency, funneling or history of cervical insufficiency.

\section{Methods}

In this study, 58 cases of 60 patients who underwent emergency cervical cerclage (ECC) between April 2014 and May 2019 were reviewed retrospectively. All data of the patients were obtained from hospital files and special records of perinatologist ultrasonography. Emergency cervical cerclage was divided into two groups as funneling (partially dilatation of cervix) 29 and complete cervical patency 29. The demographic and clinical data of the cases and survival were determined. All patients underwent fetal and cervical examinations by GE's Voluson E-8 device by a single perinatologist. Cervical evaluation was confirmed by transvaginal sonography (TVS) and gentle speculum examination. After informed about the possible risks, informed consent was obtained and cervical cerclage was applied under general or local anesthesia at the operating room conditions. Mersilene tape suture was used for cerclage in cases with full cervical patency and supported with prolene suture, mersilene tape suture was used only in other cases. All patients were given prophylactic antibiotic treatment and also tocolytic and analgesic treatment were if necessary. Patients were mobilized from the first postoperative day. After this treatment, patients were discharged from the hospital for a follow-up visit every two weeks. In addition, all patients were contacted by telephone to obtain information about their latest status.

\section{Results}

The mean age of 58 patients who underwent cervical cerclage was $29.7 \pm 5.87$ (18-48), gestational age $20.16 \pm 3.90$ (10-30), gravida $2.40 \pm 1.51$ (1-8), parity $0.74 \pm 1.19(0-7)$, abortion $0.83 \pm$. $1.19(0-4)$ and the number of living children was $0.37 \pm 0.84(0-5)$. Of the patients, 49 (68.96) were pregnant by spontaneous, 10 (14.9\%) by IVF, 8 (12\%) by ovulation induction, $14(24.13 \%)$ were twin pregnancies, $1(1.72 \%)$ was triplets pregnancy. The mean gestational week at the time of cervical cerclage was found $20.34 \pm 4.10(10-30)$ in all cases, $19.48 \pm 5.06$

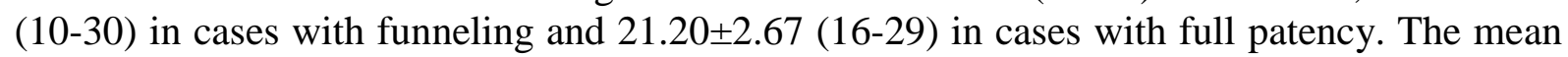
gestational age at birth was $32.03 \pm 6.66(16-40)$ in all cases, $33.37 \pm 6.30(19-40)$ in cases with funneling and $29.74 \pm 6.74$ (16-39) in cases with full patency. Of all cases, 15 (25.86\%) had multiple pregnancies (one triplet) and 43 (74.13\%) had single pregnancies. Of all cases who had cervical cerclage and whose pregnancy terminated, funneling 24 (82.75\%), 17 (58.62\%) full patency, 41 (70.68\%) pregnancies and 52 (70.27) babies survived and 2 (3.44\%) pregnancies continue. The mean weight of all live fetuses 2285 \pm 1023 (650-3900), in funneling cases 2088 \pm 1122 (270-3900) and in cases with full opening $1513 \pm 999$

\section{Conclusion}

In our study, $71 \%$ of pregnant women with full cervical patency and funneling (partially dilatation) provided the survival of pregnancy with emergency cervical cerclage. Although the chances of success of cerclage is decreased in patients with full cervical patency and multiple pregnancies, we think that cervical cerclage should be performed by experienced surgeons after detailed information and informed consent is given to these patients. 


\title{
E1485 - A NOVEL TECHNIQUE FOR PREDICTION OF PRETERM BIRTH FETAL NASAL DOPPLER FOR BREATHING PATTERNS
}

\author{
Esin S. ${ }^{1}$, Okuyan E. ${ }^{2}$, Tohma A. ${ }^{3}$
}

Baskent University, Perinatology, Ankara-Turkey ${ }^{1}$

Batman Maternity Hospital, Obstetrics, Batman-Turkey ${ }^{2}$

Baskent University, Obstetrics, Ankara-Turkey ${ }^{3}$

\section{Objective}

Technical advances during the last decades on ultrasonography and Doppler investigations have greatly facilitated research into fetal physiology but its translation into clinical practice has been limited. Breathing movements of the fetus have been demonstrated in many mammalian species. Besides that it appears to be a part of normal fetal development, absence of fetal breathing has been found to be a good predictor of preterm delivery in symptomatic patients. However, fetal breathing patterns for preterm birth prediction have not been investigated before. We hypothesized that if fetal breathing decreases before birth, and then there should specific fetal breathing patterns predictive for preterm birth such in the case of Kussmaul breathing for diabetic ketoacidosis. Therefore, in this prospective study, we aimed to investigate fetal breathing patterns in symptomatic preterm labor patients.

\section{Methods}

This study was a multicenter, prospective cohort study. Singleton pregnant patients aged 18 to 45 years old between 24-37 gestational weeks diagnosed with preterm labor were included in the study. Preterm labor was diagnosed if patient had regular uterine contractions and cervical dilation was $\geq 2 \mathrm{~cm}$. For eligible patients during routine admission ultrasonography, fetal breathing was assessed with two dimensional and Doppler ultrasonography. Fetal nostril width was measured. Fetal breathing was checked by both visual inspection of fetal diaphragmatic movements and by Color Doppler of fluid movements through fetal nostrils.

\section{Results}

During the study period, 241 patients were recruited and 73 patient results were available for the final analysis. During investigation, 3 patterns of fetal breathing was noticed: 1. No fetal breathing was present, Group 1 (N=12, 16.4\%) 2. Fetal breathing is present with regular inspiration and expirations and Doppler analysis was performed, Group 2 ( $\mathrm{N}=52,71.2 \%)$

3. Fetal breathing is present but inspiration and expirations were irregular and no Doppler analysis was possible, Group 3(N=9, 12.3\%)

$11(91.7 \%)$ of patients with no fetal breathing (Group 1) whereas $26(42.6 \%)$ of those who had fetal breathing (Group 2 and Group 3) delivered in the first 24 hours $(p=0.003)$. When deliveries in the first 48 hours were compared there was still difference between groups ( 11 (91.7\%) vs. 30 (49.2\%), $p=0.009)$.

For group 2 patients, inspiration/expiration ratio was different between those who delivered in the first 24 hours $(\mathrm{p}=0.042)$. The area under curve was 0.682 in the ROC curve. The Youden index was 1.23 for inspiration/expiration ratio.

For patients with no fetal breathing or for patients with breathing but inspiration/expiration ratio is less than 1.23 sensitivity and specificity of prediction of delivery in the first 24 hours was $94.6 \%$ and $42.4 \%$, respectively. 


\section{Conclusion}

This study is the first in the literature about investigating fetal breathing patterns with Doppler ultrasonography and according to its results $94.6 \%$ of patients who will deliver in the first 24 hours may be detected by using Doppler ultrasonography. Although absence of fetal breathing is the main factor predicting preterm birth, inspiration/expiration ratio is also important and may easily be used to predict preterm birth. 


\section{Obstetrics - Perinatal infections}

E1080 - MENINGITIS IN PREGNANCY

García Francés S. ${ }^{1}$, Urtasun Murillo M. ${ }^{1}$, Abian Franco N. ${ }^{2}$, Gastón Moreno B. ${ }^{1}$, Ruiz García M. ${ }^{1}$, Bazán Legasa M. ${ }^{1}$, Barrenetxea Arrinda J. ${ }^{1}$, Zabaleta Loinaz I. ${ }^{1}$, Pedroarena Lecumberri I. ${ }^{1}$, Pérez Munarriz B. ${ }^{1}$

Complejo Hospitalario Navarra, Obstetrics, Pamplona-Spain ${ }^{1}$

Hospital Reina Sofía, Obstetrics, Tudela-Spain ${ }^{2}$

\section{Case}

Pregnant woman 20 weeks of gestation, goes to the emergency room for frontal, pulsatile and very intense headache. She doesn't have photophobia or sonophofia. She has a temperature of $38^{\circ} \mathrm{C}$ upon arrival at hospital. She doesn't show visual, language or mobility alterations. No nausea or vomiting. No intestinal transit alterations. The patient has a history of gastroenteritis in her daughter a week ago. The neurological exploration is normal. Upon suspicion of meningitis, a blood test, blood and urine culture and a lumbar puncture are performed. Empirical antibiotic treatment is started with ceftriaxone, ampicillin and vancomycin. The patient is admitted for treatment and surveillance. The cultures are positive for Enterovirus, being diagnoses with viral meningitis. Antibiotic treatment is suspended and symptomatic headache treatment is performed during admission. Fetal control ultrasounds are performed during hospital admission. The patient remains hospitalized during 8 days with gradual improvement of symptoms. There is no obstetric complications of bleeding, contractions, etc during admission and at all time fetal well-being is confirmed. At hospital discharge, the patient is follow up in a high-risk obstetric clinic. Pregnancy and delivery after discharge are normal.

\section{Discussion}

Other than an increased risk of paralytic poliomyelitis reported in the 1950s, enterovirus infections have not generally been associated with severe outcomes in pregnant women. Although rare cases of intrauterine fetal death are reported, experimental data suggest that the placental barrier reduce risk of fetal infection. This finding may explain the paucity of evidence linking developmental abnormalities to maternal enterovirus infection. The risk of complications of pregnancy are greatest when infection occurs near term. Sudden onset of fever and severe abdominal pain mimicking abruption placentae and attributed to mesenteric adenines as well as intrauterine death have been reported with maternal echovirus late in pregnancy. In addition, there is a substantial risk of vertical transmission to the newborn infant when maternal infection occurs in the peripartum period.

\section{Conclusion}

Viral meningitis by enterovirus is an infrequent infection with little impact during pregnancy but requires fetal and maternal close monitoring and control. 


\section{E1125 - TO THE QUESTION OF BILIARY ATRESIA IN CHILDREN WITH CMV INFECTION}

Kizatova S. ${ }^{1}$, Tukbekova B. ${ }^{1}$, Dyussenova S. ${ }^{1}$, Kiryanova T. ${ }^{1}$, Erimbetova N. ${ }^{1}$

"Karaganda Medical University", Department "Children Diseases №2", Karaganda

Kazakhstan $^{1}$

\section{Objective}

To study the clinical and laboratory features of the course and prognosis of biliary atresia in newborns with congenital CMV infection

\section{Methods}

On the basis of the Karaganda Regional Children Clinical Hospital, during 8 years (from 2010 to 2018), 14 patients with atresia of biliary duct with congenital CMVI were examined and treated. The identification of atresia of the biliary tract was based on the assessment of pregnancy period, an early history of the child's life, the results of biochemical studies and instrumental methods of research.

\section{Results}

Prenatal congenital malformations in fetuses were not identified. During pregnancy, chronic CMV infection in mothers was diagnosed by PCR in $21 \%$ of cases.

Dates of manifestation of pathological jaundice in newborns in $28 \%$ of cases were noted from birth, in other cases from 1 month and older, with jaundice gradually becoming more intense, acquiring a greenish tint. The stool was discolored from the moment of birth in $15 \%$, from the late neonatal period in $85 \%$ of children. It is important to note the persistent nature of this symptom. Urine on the background of the discolored stool was a dark color. At admission in children from 1.5 months of age there was marked hepatosplenomegaly. In the late stages of the disease (by 5-6 months), which were noted in $28 \%$ of cases, the abdomen increased, the network of saphenous veins of the abdominal wall expanded, ascites and hemorrhagic phenomena developed, which is explained by the development of biliary cirrhosis due to a violation of bile flow. When examined by the PCR method of blood, urine and saliva, in 35\% of cases CMVI DNA was found, positive markers of hepatitis B and C were found in 1 case, in $42.8 \%$ of cases mixed infections were detected (CMVI in combination with mycoplasma or toxoplasma, or with ureaplasma). Biochemical analysis revealed a moderate increase in the cytolytic activity of the enzymes: ALT $150.8 \pm 22.6 \mathrm{U} / \mathrm{l}$, AST $240.2 \pm 36.6 \mathrm{U} / \mathrm{l}$. The level of total bilirubin increased to $153.7 \pm 17.6 \mu \mathrm{mol} / 1$, with the predominance of the direct fraction of bilirubin $(84.0 \pm 10.1 \mu \mathrm{mol} / 1)$. According to an ultrasound scan, computed tomography, a visual impairment of the intrahepatic bile ducts and gallbladder with ABT was detected in $79 \%$ of infants, cystic atresia of the choledoch in $14 \%$, and gall bladder dropsy in $7 \%$ of cases. In the first half of the year, 4 (28.5\%) children were taken for surgical treatment. The remaining children, in connection with the current CMVI, continued treatment of the underlying disease, death occurred in the first year of life in most children.

\section{Conclusion}

Early prenatal diagnosis of fetal infection and biliary atresia allows timely surgical correction, and increase patient survival. In general, the prognosis of bile duct atresia is very serious, since cirrhotic changes in the liver are progressive. 
E1151 - PYELONEPHRITIS COMPLICATED WITH ARDS PNEUMONIA AND SEPSIS DURING THIRD TRIMESTER OF PREGNANCY CASE REPORT

Aur C. ${ }^{1}$, Copos A. ${ }^{1}$, Cuc R. ${ }^{1}$, Mascas G. ${ }^{1}$, Mocuta D. ${ }^{1}$

Spitalul Clinic Judetean De Urgenta Oradea, Obstetrics-Gynecology, Oradea-Romania ${ }^{1}$

\section{Introduction}

Pyelonephritis is the most common non-obstetric antepartum infection which requires hospitalisation because of then high incidence of the maternal and neonatal morbidity and mortality. Pregnant woman with pyelonephritis are increase risk for server infection complication such as sepsis, ARDS, pneumonia , acute heart and renal failure which require I.C.U admission.

\section{Case}

We present the case of a 38 years old pregnant woman admitted in our hospital for flue like symptoms (fewer, cough, dyspnea) and urinary symptoms.

Obstetrical ultrasound highlighted a 30 weeks singleton pregnancy with no abnormalities in Doppler fetal-velocimetry. Personal and obstetrical history are not significant. Because of the sever dyspnea, hypotension and tachycardia which appeared 12 hours after admission, she was transferred to I.C.U with the diagnosis of pneumonia , ARDS and sepsis, complicating a pyelonephritis. After a few hours she has still an altered status which is also associated with generalized rash.

\section{Discussion}

Paraclinical monitoring put in evidence high level of WBC and CRP, uricemia, hyperglycemia, acidosis with base deficit ( ph 7,30 and base deficit -14,3mmol/l) and interstitial pneumonia which was diagnosed on clinical and radiologic aspect. Due to blood culture and urinary culture the diagnosis is pyelonephritis with Escherichia Coli. Also the patient was tested for flue with RIDTs and culture from the sputum, the results were negative for any other infectious diseases.

During 48 hours of interdisciplinary team (ob-gyn, anesthetist, nephrologist, pneumologist and infectiologist doctor) it was initiated an empiric treatment with large broad iv antibiotics which was later adjusted after antibiogram.

Under antibiotic therapy, oxygen therapy and bronchodilators medication, the general status of the patient is slowly adjusting with improvement of clinical and paraclinical parameters . In the next 72 hours she was discharged for I.CU, after 5 days of iv Cephalosporin (according to the ABG). The patient delivered vaginally after 8 weeks, a male newborn baby, 2900 gr, IA $9 / 10$.

\section{Conclusion}

In summary, this case report is about a third trimester pregnancy associates with pyelonephritis which was complicated with pneumonia, ARDS, sepsis and was successfully managed in multidisciplinary team. 


\section{E1178 - EXPECTATIVE MANAGEMENT FOR ECTOPIC PREGNANCY}

Bohiltea.$^{1}{ }^{1}$, Teodor O. ${ }^{1}$, Turcan N. ${ }^{1}$, Baros A. ${ }^{1}$, Cirstoiu M. ${ }^{1}$

University Emergency Hospital Bucharest “Carol Davila” University of Medicine and

Pharmacy, Bucharest, of Obstetrics and Gynecology, Bucuresti-Romania ${ }^{1}$

The ectopic pregnancy accounts for up to $2 \%$ of all reported pregnancies. The main detection tools for ectopic pregnancy are serum beta-human chorionic gonadotropin ( $\beta$-hCG) measurement and vaginal ultrasonography techniques. The treatment options involve surgical management preferred for the life threatening conditions, as well as conservative treatment for the uncomplicated cases. The guidelines for timely diagnosis and appropriate management are constantly updated, but compared to the medical treatment protocols, the indication for expectant management based on the $\beta$-hCG level remains inexplicit. In this paper, based on clinical experience with spontaneous resolution of the early ectopic pregnancy, we investigated the correlation between the serum $\beta$-hCG measurements and the outcome of the expectative approach. We retrospectively assessed the charts of all the patients diagnosed with ectopic pregnancy, who were hospitalized in the Department of Obstetrics and Gynecology of the University Emergency Hospital Bucharest between 1st January 2014 and 31 December 2018. The curative methods used for the patients enrolled in the study were evaluated in order to determine the impact of the expectative approach. During the five years, in our department were hospitalized 440 patients diagnosed with ectopic pregnancy. Although conventional surgical treatment was still the most widely used treatment, the expectative attitude was initiated for 114 cases. Based on $\beta$-hCG dynamic values, were selected the cases that required abandoning the initial approach and were suitable for further medical treatment with methotrexate. This management strategy allowed avoiding overtreatment. Closely clinical and paraclinical monitoring was required. Both the initial level and the dynamics of $\beta$-hCG values, beside the seriated Doppler transvaginal ultrasound evaluations, represented important guiding tools for the therapeutic decision. With appropriate and prompt management, maternal mortality and morbidity due to ectopic pregnancy can be reduced. Every patient diagnosed with this condition requires a strictly individualised management. The expectative should not be underestimated, as it is important and efficient for stable patients in specific circumstances. The low rate of failure or complications related to the expectant management suggests the benefit of this alternative, avoiding the risks of medical treatment. 


\section{E1212 - PREGNANCY OUTCOME IN GENITAL TRACT INFECTION ASSOCIATED WITH DIABETES}

Aur C. ${ }^{1}$, Mocuta D. ${ }^{2}$, Copos A. ${ }^{1}$, Cuc R. ${ }^{1}$, Mascas G. ${ }^{1}$

County Emergency Clinical Hospital, Ob-Gyn, Oradea-Romania ${ }^{1}$ County Emergency

Clinical Hospital, Ob-Gyn, Oradea-Romania ${ }^{2}$

\section{Objective}

It is well known the high maternal and fetal risk for complications in pregnancy associated with diabetes. Genital tract infection has a more increased incidence in pregnancy associated with diabetes. The goal of our study was to assess the frequency and the type of complications which can appear at the pregnant patients who were diagnosed with diabetes and antepartum genital tract infection.

\section{Methods}

This is a retrospective study, about the pregnant women with preexistent or gestational diabetes who delivered in our obstetric department in a period of 24 months (1st of January 2017 - 31st of December 2018). Vaginal secretion was sampled from all the patients and microbiological study was performed. The pregnancy outcome was analyzed in relation with the presence or the absence of genital tract infection.

\section{Results}

During the last 2 years, 7194 women delivered in our hospital. From these, 48 cases were monitored during pregnancy because of their high glycemic values. In this group, for 17 patients the diabetologist recommended insulin therapy. Among pregnant women with diabetes admitted in the delivery room, abnormal vaginal flora was identified at 27 patients, which means more than half of them. E. Coli was present in 19 cases (39,58\%), Klebsiella pneumoniae, Proteus mirabilis and Gardnerella vaginalis appeared each in 2 cases (4,16\%). Group B Streptococcus and Enterobacter appeared each of them in 1 case (2,08\%). Most of the cases had vaginal candidiasis, too.

Cases with diabetes and genital tract infections had a high incidence of material and fetal complications comparing with cases without infection. The most frequent complications we can mention are: premature births in 15 cases (31,25\%), retroplacental hematoma in 11 cases $(22,91 \%)$ and the delivery finalized by C-section in 43 cases (89,58\%). Neonatal complications included hypoglycemia, hyperbilirubinemia, hypocalcemia and neurological damage especially in the premature new born babies (in 2 cases).

\section{Conclusion}

Genital tract infection in patients with diabetes was highly associated with poor perinatal outcome. It is very important to perform the microbiological screening in these pregnancies. Controlling the genital infection and the level of glycemia in these patients could improve the outcome. 


\section{E1222 - CASE REPORT CHALLENGING CMV INFECTION DIAGNOSIS COMPLICATED BY CONGENITAL HEART DISEASE}

Yatrakou E. ${ }^{1}$, Karachristou K. ${ }^{1}$, Giannakopoulou A. ${ }^{2}$, Lappa A. ${ }^{1}$, Liouli C. ${ }^{1}$,

Karanasios E. ${ }^{2}$, Papaevangelou V. ${ }^{3}$, Anatolitou F. ${ }^{1}$

Ag Sophia Childrens Hospital, A, Athens-Greece ${ }^{1}$

Ag Sophia Childrens Hospital, Cardiology, Athens-Greece ${ }^{2}$

General University Hospital Attikon, 3rd Department of Pediatrics, Haidari-Greece ${ }^{3}$

\section{Introduction}

CMV infection of a neonate is an important health problem worldwide. Although not typically associated with structural heart disease it can complicate the management and prognosis of neonates requiring cardiac surgery. We present two such cases cared for in our NICU.

\section{Case}

A 29 day old full term male infant was transferred to our unit with a postnatal diagnosis of Trisomy 21 and atrioventricular septal defect. He was in heart failure upon admission and in need of inhaled oxygen up to $30 \%$, which stabilized with diuretics. The infant was born to an underage mother with no antenatal care. Maternal CMV status was unknown, as was the neonatal CMV status. Routine CMV serology was reported as IgM (+) and IgG (+). The result was confirmed with blood and urine CMV positivity by PCR detection, while CSF was negative for the virus. Brain U/S scan, ophthalmologic examination, liver serology and FBC were within normal limits. The baby passed aABR testing for both ears. To distinguish between congenital and acquired CMV infection we utilized blood taken for the Guthrie card at 3 days of age. DNA extraction from the dried blood spot was performed and tested negative for CMV by PCR, the infant was therefore diagnosed with an acquired CMV infection and did not receive antivirals.

Our second neonate was also a full-term male who was admitted in the first day of life with a prenatal diagnosis of TGA. Maternal CMV status at 12 weeks was IgM (-) and IgG (+), but had not been further tested during the pregnancy. Cardiac echocardiography confirmed transposition of the great arteries, PDA, VSD and a small patent foramen ovale. Due to pulmonary hypertension the neonate had severe cardiovascular instability, received blood products from the first day of life and needed both inotrope support and HFOV with NO. He had cardiac catheterization and Rashkind atrial septostomy at 7 days of life, which stabilized him enough to have a successful arterial switch operation utilising cardiopulmonary bypass. Post-operatively the baby was nursed in the cardiac ICU and returned to our unit at 33 days of life. CMV testing performed on day 1 had shown $\operatorname{IgM}(+)$, IgG(+) for CMV, however by the time of results reporting the neonate had already been admitted for cardiac surgery, had been transfused several times and further CMV testing was considered unreliable. Platelet count, liver serology, cranial U/S scan, ophthalmologic exam and aABR were again normal, postsurgery. To ascertain whether the infant had CMV infection we used again blood from the Guthrie card taken at 3 days of age. DNA extraction from the dried blood spot was performed and tested negative for CMV by PCR, the infant was therefore presumed not to have CMV infection and received no antivirals.

\section{Conclusion}

As CMV infection is ubiquitous it should be screened for actively during pregnancy and in any neonate when maternal serology is non-existent or incomplete. Novel detection methods can aid in uncertain cases and guide need for antiviral treatment and follow-up plans. 


\author{
E1257 - E.COLI PYELONEPHRITIS COMPLICATED WITH SEPSIS DURING \\ THIRD TRIMESTER OF PREGNANCY A CASE REPORT \\ Copos A. ${ }^{1}$, Aur C. ${ }^{1}$, Mocuta D. ${ }^{1}$, Mascas G. ${ }^{1}$, Negrini R. ${ }^{1}$ \\ County Emergency Clinical Hospital of Oradea, Obstetrics, Oradea-Romania ${ }^{1}$
}

\title{
Introduction
}

Pyelonephritis is the most common non-obstetric antepartum infection which requires hospitalization because of the high incidence of the maternal and neonatal morbidity and mortality.

Pregnant women with pyelonephritis are at increased risk for severe complications such as sepsis, ARDS (Acute Respiratory Distress Syndrome), pneumonia, acute heart and renal failure, which often could require Intensive Care Unit (ICU) admission. We present here the case of a multiparous 38 years old pregnant woman admitted in our hospital for urinary symptoms associated with flu-like symptoms (cough, rhinorrhea and dyspnea).

\section{Case}

Obstetrical ultrasound highlighted a 30 weeks singleton pregnancy with no abnormalities in fetal growth and in fetal Doppler velocimetry. Personal and obstetrical history was not significant.

Because of the high fever, sever dyspnea, hypotension and tachycardia which appeared 12 hours after admission she was transferred to ICU with the suspicion of sepsis, pneumonia and ARDS. After a few hours a generalized rush appeared and she still had fever and an altered status. Paraclinical monitoring put in evidence high level of leucocytes and CRP, acidosis with base deficit (ph 7,30 and base deficit $-14,3 \mathrm{mmol} / \mathrm{l}$ ), hyperglycemia and uricemia. Interstitial pneumonia was diagnosed based on clinical and radiological aspects. Blood and urinary cultures were obtained. The later results showed they were both positive for E. Coli. The diagnosis was pyelonephritis and sepsis with Escherichia Coli complicated with pneumonia and ARDS. The patient was also tested for AH1N1 and the cultures from the sputum were negative. The multidisciplinary team ( ob-gyn, anestesiologist, nephrologist, pneumologist and infectiologist) recommended from the beginning large broad spectrum iv antibiotics. After 72 hours of antibiotherapy, oxygen therapy and bronchodilators, the general status of the patient was improved, with normalization of clinical and paraclinical parameters. She was than discharged from ICU. Orally antibiotherapy according to the antibiograms was continued after for another more 5 days.

The evolution of the pregnancy was a normal one till the 38-th week of pregnancy, when the patient delivered vaginally a male newborn baby, 2900 gr, Apgar 10.

\section{Conclusion}

This case report is about a pyelonephritis with $\mathrm{E}$ coli in third trimester of pregnancy complicated with pneumonia, ARDS and sepsis, which was successfully managed in multidisciplinary team. 


\section{E1308 - VIVAX MALARIA IN PREGNANT WOMAN WITH GESTATIONAL DIABETES IN A HOSPITAL OF THE AMAZON REGION CASE REPORT}

Ribeiro Simões M. ${ }^{1}$, Gloria Da Silveira E. ${ }^{2}$, Simões Lamego L. ${ }^{3}$, Simões Lamego G. ${ }^{4}$, Ribeiro Freitas P. ${ }^{5}$, Pandolfi Freitas T. ${ }^{1}$

Faculdades Integradas Aparicio De Carvalho, Medicina, Porto Velho-ro-Brazil ${ }^{1}$

Universidade Federal De Rondônia, Geografia, Porto Velho-ro-Brazil ${ }^{2}$

Hospital Getúlio Vargas, Urgência E Emergência, Tramandaí-rs-Brazil ${ }^{3}$

Gso Medicina Ocupacional, Medicina Do Trabalho, Esteio-rs-Brazil ${ }^{4}$

Clinica Maria Do Carmo, Ultrassonografia, Porto Velho-ro-Brazil ${ }^{5}$

\section{Introduction}

Malaria is one of the most important infectious diseases worldwide. It occurs in more than 90 countries, putting at risk about $40 \%$ of the population. Its control requires an integrated approach, including prevention and immediate treatment with antimalarials. Malaria is caused by parasites of the genus Plasmodium that are transmitted to people by the bite of females infected with mosquitoes of the genus Anopheles. Plasmodium vivax is the most common in Brazil. Its symptoms are fever, headache, chills and vomiting. The pregnant woman mainly causes abortion, premature birth, anemia, intrauterine growth restriction and death.

\section{Objective}

To report a case of vivax malaria in a pregnant woman with gestational diabetes occurring in a hospital in the Amazon region and to review literature of greater relevance on the subject

\section{Methods}

The present work uses a bibliographical research reference, in order to seek information and report the case on Plasmodium Vivax infection during pregnancy in a Hospital in the Amazon region.

\section{Case}

AALC, 34years, pregnant, 13s1d, G5PC3A1, attended for attendance at the obstetric center on 05/05/2017, referring treatment for malaria vivax. Physical examination at normal admission, blood pressure 110/70 mmHg, TOTG 75g 96\148\99. Examination: uterus with $12 \mathrm{~cm}$ in height, heart beats present: 140. Requested examination of plasmodium and obstetric ultrasonography. Confirmed the diagnosis of infection by plasmodium vivax $+++/ 4+$, initiated treatment with chloroquine $150 \mathrm{mg}$ scheme $4 / 3 / 3$ tablets and sent to the Pre-Natal of High Risk. On 09/15/2017 returns with IG 32S1D to perform glycemic profile, makes use of chloroquine 150mg 2 tablets weekly. Examination: uterus with $32 \mathrm{~cm}$ high, heart rate 147.Unrealized vaginal tap. Was confirmed the diagnosis of gestational diabetes and USG polihidramnia with normal Doppler. The patient was admitted to the obstetric center of HBAP, where cesarean delivery occurred with 40S1D.With live RN, male, weighing 3960 gr, $46 \mathrm{~cm}$ of height. There were no malformations. Diagnosis and early treatment of malaria reduce maternal-fetal complications, avoiding maternal death and neonatal damage. Therefore, it can be considered that all pregnant women should be targeted for malaria prevention and control actions.

\section{Conclusion}

Diagnosis and early treatment of malaria reduce maternal-fetal complications, avoiding maternal death and neonatal damage. Therefore, it can be considered that all pregnant women should be targeted for malaria prevention and control actions. 


\section{E1344 - CERVICAL INTRAEPITHELIAL NEOPLASIA AND ITS DEVELOPMENT DURING PREGNANCY IN PATIENTS FROM MACEDONIA}

Dzikova E. ${ }^{1}$, Dimitrov G. ${ }^{1}$, Tofoski G. ${ }^{2}$, Daneva Markova A. ${ }^{3}$, Aleksioska I. ${ }^{4}$

University Clinic for Gynecology and Obstetrics, Gynecologic Oncology, Skopje

Macedonia ${ }^{1}$

University Clinic for Gynecology and Obstetrics, Infertility and Assisted Reproduction, Skopje-Macedonia ${ }^{2}$

University Clinic for Gynecology and Obstetrics, High Risk Pregnancy, Skopje-Macedonia ${ }^{3}$ University Clinic for Gynecology and Obstetrics, Urogynecology, Skopje-Macedonia ${ }^{4}$

\section{Objective}

The study was conducted to evaluate the development of cervical intraepithelial neoplasia (CIN) during pregnancy compared to non-pregnant-women.

\section{Methods}

We have analyzed 50 patients, 25 pregnant and 25 non- pregnant, in the University clinic for gynecology and obstetrics and Private Health Institution "Dr. Emilija Trajkovska”. We have evaluated the rates of persistence, progression and regression of CIN conferring to histological findings in the initial visit and after delivery. The results were compared with the non-pregnant group of women followed up for nine months.

\section{Results}

The study was consisted of 50 pregnant women with cervical intraepithelial. Low grade and High grade cervical intraepithelial neoplasia were histologically proved in $34 \%$ and $66 \%$ of all pregnant women, respectively. According to the histological findings after delivery cervical intraepithelial neoplasia showed a considerably higher affinity to spontaneous regression $(56 \%$ vs. $64 \%$ patients $p=0.010)$ On the other hand, the persistence rate was significantly lower than in non-pregnant women $(40 \%$ vs. $58 \%$ patients, $p=0.048)$. The progression rate was very low in pregnant women.

\section{Conclusion}

The regression rate of cervical intraepithelial neoplasia is significantly high after delivery. For that reason, the decision for the definite type of treatment should be made according to the findings after delivery. On the other hand, the time and the method of treatment should be made according to the severity of the lesion. 


\section{E1373 - HERBAL COMBINATION TREATMENT OF THE RENAL DISORDERS DURING THE PREGNANCY}

Kozub T. ${ }^{1}$

V. N. Karazin Kharkiv National University, Obstetrics and Gynecology, Kharkiv-Ukraine ${ }^{1}$

\section{Objective}

The gestation course is often complicated with the UTIs - from asymptomatic bacteriuria to pyelonephritis. Treatment of the renal disorders during the pregnancy is associated with the significant difficulties. Using the herbal products during the pregnancy is very essential. Preference is given to the ready-to-use combination products that exert anti-inflammatory and antibacterial action, have diuretic, litholytic, spasmolytic effects.

\section{Methods}

All patients were randomly divided into two clinical groups: - Group 1 included 47 women with the acute uncomplicated lower urinary tract infection (cystitis), who received standard treatment for 10 days. The standard scheme included the antibacterial products based on the applicable legislation of Ukraine. Investigator selected the treatment;

- Group 2 included 53 women with the acute uncomplicated lower urinary tract infection (cystitis). The patients from that group received standard treatment based on the applicable legislation of Ukraine. The investigator selected the treatment scheme. The standard treatment in this group also included herbal products (Sper gulariarubra, Peumusboldus, Opuntiafi cusindica, Sideritisangustifolia, Rosmarinusoffi cialis, Cynodondactylon, Melissa Offi cinalis) for 10 days.

All patients had the complete clinical, laboratory and instrumental examination.

\section{Results}

Monitoring of the patients has demonstrated the significant differences in the therapy efficacy. Patients reported the clinical improvement and reduction of complaints on Day 3.0 \pm 0.12 and $1.8 \pm 0.09$ of disease in group 1 and 2 , respectively. It should be noted that improvement and relief of the clinical symptoms were more rapid in the patients who received combination treatment. The follow-up urine analysis on Day 10 has shown the change in urine $\mathrm{pH}: 5.61 \pm$ 0.09 in group 1 and $6.41 \pm 0.06$ in group 2. These findings are indicative of the urinary alkalinizing.

The analysis of the clinical and laboratory findings of the patients with acute uncomplicated lower urinary tract infection (cystitis) before and after the treatment has demonstrated the significant differences in the clinical symptoms of women from group 1 and 2 . In group 2, on Day 10 of therapy, proteinuria was persistent in $3.78 \%$ and crystalluria in $1.88 \%$ of women. The clinical and laboratory findings of group 1 have demonstrated worse results: proteinuria was persistent in $8.51 \%$ of women, crystalluria - in $8.51 \%$, dysuria was observed in $34.04 \%$, abdominal pain - in $23.4 \%$, bacteriuria - in $10.63 \%$, leukocyturia in $8.51 \%$, discoloration of urine - in $2.12 \%$. At the same time, those symptoms were absent in women from group 2 after the combination therapy. No patients from group 2 were presented with the repeated complaints within 6 weeks after treatment completion. Seven patients from group 1 (3\%) reported the disease recurrence and were subject to the repeated treatment.

\section{Conclusion}

The conducted study has demonstrated that herbal combination products used in the pregnant women with the acute uncomplicated lower urinary tract infection (cystitis) with the 
concomitant crystalluria is an effective, safe and clinically relevant due to the marked uroseptic, spasmolytic, diuretic, litholytic, anti-inflammatory effects. It offers more rapid clinical response in the treatment of cystitis and crystalluria and ensures the preventions of pyelonephritis and edemas in the pregnant women. 
E1414 - HEALTH CARE ASSOCIATED INFECTIONS AND ANTIMICROBIAL RESISTANCE PATTERNS IN A TERTIARY NEONATAL INTENSIVE CARE UNIT OVER FIVE YEARS IN TURKEY

Memisoglu A. ${ }^{1}$, Kepenekli Kadayifci E. ${ }^{2}$, Culha G. ${ }^{3}$, Ozdemir H. ${ }^{1}$, Soyletir G. ${ }^{4}$, Bilgen $H^{1}$, Ozek E.

Marmara University, Neonatology, Istanbul-Turkey ${ }^{1}$

Marmara University, Pediatric Infectious Diseases, Istanbul-Turkey ${ }^{2}$

Marmara University, Hospital Infection Control Committee, Istanbul-Turkey ${ }^{3}$

Marmara University, Microbiology, Istanbul-Turkey ${ }^{4}$

\section{Introduction}

Healthcare-associated infections (HCAI) are one of the risks which increase neonatal mortality, morbidity, and health care cost in neonatal intensive care units (NICU). As the characteristics of HCAI's vary considerably in different regions and over time, continual HCAI surveillance is important to determine HCAI's rates and healthcare associated pathogens for infection control.

\section{Methods}

We aimed to analyze the characteristics of HCAI in a tertiary referring centre in Turkey. All newborns admitted to the NICU between January 2014 and December 2018 were included in this study. HCAI related information has been collected retrospectively according to the "National Hospital Infection Surveillance Network" and the standards of Centers for Disease of Control and Prevention.

\section{Results}

This descriptive study was conducted by active search of infection records among newborns in the NICU of Marmara University Hospital. The neonatal intensive care unit with 14 beds has an occupancy rate of $100 \%$ and offers care to critically ill newborns, extremely low birth weight premature infants, neonates requiring pre- or postoperative management, and those who have congenital anomalies that require close observation or intervention. Nurse/baby ratio has been $1 / 3$ in the study period. Total patient hospitalization day was 24,161 throughout the study. Of the 1395 patients hospitalized in the NICU, 265 were diagnosed with infection (infection rate 19\%; infection density 11/1000 patients days). Of the 316 samples sent, 75 did not produce growth. Out of isolated microorganisms, $47.5 \%$ were Gram negative, $24.4 \%$ were Gram positive bacteria, $2.5 \%$ were yeasts and viruses were isolated in $1.27 \%$ of them. The most frequently isolated Gram negative bacteria were Klebsiella spp. (16.7\%) and Acinetobacter spp. (8.6\%), Pseudomonas spp. (6.3\%), Escherichia coli (4.1\%) and Enterobacter spp. (3.2\%). About ninety six percent of Acinetobacter baumanii were meropenem resistant; carbapenem resistance of Enterobacter spp were $0 \%$. More than $80 \%$ of Pseudomonas aeruginosa were resistant for ceftazidime and amikacin. There were $90.6 \%$ carbapenem resistance of Klebsiella pneumoniae and $83.3 \%$ amikacin resistance of Escherichia coli. The most commonly isolated Gram positive bacteria were coagulase negative staphylococci (CNS) (13.6\%), Staphylococcus aereus (6.7\%) and Enterococcus spp. (3.1\%). In CNS strains, methicillin resistance was found to be 3.9\%; resistance were $47.8 \%$ for clindamycin $79.3 \%$ for trimethoprim-sulfamethoxazol. All CNS strains were susceptible to vancomycin and linezolid. 


\section{Conclusion}

This study determined that HCAI still presents a serious problem in NICU. The characteristics of HCAIs vary considerably in different regions, over time and referring to the hospitals. The availability of timely and accurate epidemiological information on healthcare associated pathogens is necessary for infection control and the appropriate selection of empiric antibiotics. 
E1461 - VIRAL INFECTIONS IN PREGNANCY AND THEIR TREATMENT

Mihajlovic S. ${ }^{1}$, Nikitovic R. ${ }^{1}$, Kubat J. ${ }^{1}$, Nikolic M. ${ }^{1}$, Radovanovic M. ${ }^{1}$

Kbc Dr Dragisa Misovic, Hospital for Gynecology and Obstetrics, Belgrade-Serbia ${ }^{1}$

Nowadays we are faced with an increased number of viral infections during pregnancy. Although infections primarily leave the effects on the health of mothers, many infections can be perinatally transmitted and can cause health complications of the fetus and infant. The treatment of these infections is based on two principles, the first is the reduction of the symptoms of maternal infection and the second is the prevention of transmission of viral infection from the mother to the child.

In this, we paid special attention to the treatment of herpes simplex virus, cytomegalovirus and hepatitis B infections during pregnancy. 


\section{Obstetrics - Postpartum hemorrhage}

\section{E1065 - IMMUNE THROMBOCYTOPENIC PURPURA ASSOCIATED WITH PERIPARTUM CARDIOMYOPATHY FIRST CASE REPORT}

Martinez R. ${ }^{1}$, Villanueva J. ${ }^{2}$, Moraes A. ${ }^{2}$, Villota L. ${ }^{2}$, Constanza M. ${ }^{1}$

Pontificia Universidad Javeriana - Hospital Universitario San Ignacio, Maternal Fetal

Medicine Unit, Bogota-Colombia ${ }^{1}$

Pontificia Universidad Javeriana, Obgyn Department, Bogota-Colombia ${ }^{2}$

\section{Introduction}

Postpartum cardiomyopathy -PPCM - is a systolic heart failure that occurs in the period between the last months of pregnancy or within five months after delivery, the association with idiopathic thrombocytopenic purpura-ITP- has not been reported. Both conditions are potentially lethal and pose a challenge for the clinician to manage this complex scenario. Previous authorization of the research committee and in writing of the patient, the first case of this rare association is presented.

\section{Case}

27-year-old female patient, G1P0, of $35+5 / 7$ weeks of pregnancy, with a medical history of primary immune thrombocytopenia (IPT) since the age of 3, consulted with clinical signs compatible with preterm delivery, associated with respiratory difficulty and a saturation of $88 \%$. She is admitted with severe thrombocytopenia $(56000 / \mu \mathrm{L})$, compensated respiratory alkalosis, moderate hypoxemia and with irregular negative antibody screening. During the first six hours, the patient's condition deteriorates rapidly, requiring orotracheal intubation. At that moment, paraclinical tests evidence negative troponins and an elevated BNP. The patient is admitted in the Intensive Care Unit with ventilatory support, platelets of $6,600 / \mu \mathrm{L}$, tracheal hematic secretion, hematuria, acute kidney injury KDIGO 2 with a creatinine of $1.13 \mathrm{mg} / \mathrm{dL}$ (previous $0.47 \mathrm{mg} / \mathrm{dL}$ ), hyperlactatemia, and metabolic acidemia. Ultrafiltration and transfusion of 2 platelet concentrates (PC) are initiated. An echocardiogram is taken, which reports 37\% LVEF with a biventricular compromise and leads to the diagnosis of peripartum cardiomyopathy (PPCM).

On the second day of hospitalization, the patient was taken to an emergency cesarean, with subsequent uterine atony and the loss of approximately 2000 cc of blood, requiring noradrenaline drip, B-Lynch suture, and transfusion of $1 \mathrm{CP}$ of platelets and $1 \mathrm{pRBC}$. She continues in the ICU with vasopressors and bromocriptine is initiated. The patient presents adequate clinical evolution, on the second postoperative day extubation is achieved and on the third day, vasopressors are suspended. She is assessed by the cardiology department due to the possibility of viral or Chagas' origin myocarditis, both of which are ruled out. A control TTE is taken in the fifth postoperative day, which evidences a moderately dilated left ventricle, with severe eccentric hypertrophy with a $35 \%$ LVEF. The patient is discharged after ten days. After five months, follow-up is performed, a Stage C heart failure is evidenced with 36\% LVEF, and the patient is admitted to the heart failure clinic of the institution. Currently, the physiopathological mechanism of the PPCM is not fully understood, however, different hypotheses have been proposed about its etiology which include viral myocarditis, nutritional deficiencies, hemodynamic stresses, vascular dysfunction and angiogenic factors associated with pregnancy. The main differential diagnoses are benign dyspnea during pregnancy, severe preeclampsia, eclampsia or cardiac dysfunction due to valvular disease. Regarding treatment, it is recommended to stabilize the heart failure, loop diuretics for peripheral or pulmonary edema (3) and inhibition of prolactin because of its possible role in the physiopathogenesis of the condition (4).

In relationship with the ITP, platelet count increased in the postpartum and now she is being followed up by the hematology unit, without additional therapy. 


\section{E1078 - PRENATAL DIAGNOSIS OF PLACENTAL ABNORMALITY (PLACENTA INCRETA) CASE REPORT}

Abdalla N. ${ }^{1}$, Piórkowski R. ${ }^{1}$, Stanirowski P. ${ }^{1}$, Bachanek M. ${ }^{1}$, Cendrowski K. ${ }^{1}$, Sawicki W. ${ }^{1}$ Medical University of Warsaw, Chair and Clinic Of Obstetrics, Gynecology and Gynecological Oncology, Warsaw-Poland ${ }^{1}$

A 28-year-old patient G4P3A0 at 37th week of gestation was admitted to hospital because of onset of regular uterine contractions. She had normal uneventful vaginal delivery ten years ago and then two caesarean sections. First caesarean section was performed three years ago because of twin pregnancy. The second caesarean section was done one year later because of thin caesarean section scar in ultrasound scan and risk of uterine rupture. The patient denied chronic diseases. She is heavy smoker (more than 20 cigarettes daily). Complete placenta previa and suspicion of placenta increta was shown by ultrasound in second trimester of present pregnancy. The patient refused to be hospitalized, she was fully informed about the medical situation and possible complications.

At admission, the patient was in good general condition. The vital signs were in normal ranges. Cardiotocography showed regular uterine contractions, the tone of the uterus was normal. Fetal heart rate was reactive. Speculum examination confirmed absence of bleeding and closed cervix. Ultrasound examination showed single fetus in the longitudinal cephalic lie. The amniotic fluid index was normal. The placenta was completely covering the internal cervical orifice. The lower segment of the uterus was so thin. There were abundance blood vessels between the placenta and urinary bladder, with subjective loss of a boundary between the placenta and the urinary bladder.

The hemoglobin level was 12,3 g/dl and RBC count was 4,2 x106/ $/ \mu \mathrm{l}$. The patient was informed about the medical situation and about possible complications associated with caesarean section including severe blood loss, necessity of blood transfusion, uterine atony, blood coagulation disorders, placental abnormalities, increased risk of hysterectomy and increased risk of urinary bladder injury. Blood was crossed matched and prepared for the patient. The laboratory and intensive care unit were informed about the patient. An experienced multidisciplinary team of obstetricians and midwives performed the caesarean section. Laparotomy revealed very thin paper-like lower uterine segment with multiple enlarged blood vessels on the surface. The urinary bladder had multiple adhesions with the lower uterine segment. The adhesions were released and then a transverse uterine incision was done above the edge of the placenta and a male newborn was delivered $2680 \mathrm{~g} / 51 \mathrm{~cm}$. The placenta was centrally covering the internal orifice of the cervix. Spontaneous separation of the placenta occurred partially at its upper margin. Carbetocinum was given to the patient. Because of overt placental growth abnormality a decision of hysterectomy was taken. Hysterectomy was performed as fast as possible to minimize blood loss. Adnexa were without macroscopic lesions and they were left intact. Special care was shown to prevent injury of the urinary bladder and ureters. However, two urinary bladder injuries $(2 \mathrm{~cm}$ and $4 \mathrm{~cm})$ were noticed in the posterior wall at the sites of relieved adhesions. Bladder injuries were sutured and integrity of the walls were checked with methylene blue dye. Total blood loss was estimated as $1000 \mathrm{ml}$. The patient received two units of packed red cells. Postoperative course was uneventful. Histopathological examination confirmed placenta increta. 


\section{E1100 - MAY THURNER SYNDROME COMPLICATING PREGNANCY}

Dos Santos G. ${ }^{1}$, Pimentel L. ${ }^{1}$, Silveira S. ${ }^{2}$, Feuerschuette O. ${ }^{1}$, Carvalho M. ${ }^{1}$, Martins M. ${ }^{1}$, Garcia G. ${ }^{1}$, Gewehr M. ${ }^{1}$, Sommacal L. ${ }^{1}$, Trapani Jr A. ${ }^{3}$

Hospital Universitário/ufsc, Obstetrics, Florianópolis-Brazil ${ }^{1}$

Hospital Universitário/ Ufsc And Maternidade Carmela Dutra, Obstetrics, FlorianópolisBrazil $^{2}$

Hospital Universitário/ufsc And Unisul, Obstetrics, Florianópolis-Brazil ${ }^{3}$

\section{Objective}

May-Thurner syndrome (MTS) is a relatively common anatomical variation caused by extrinsic venous compression by the arterial system against lumbar vertebral bony in the iliocaval venous territory. The most common variant is due to compression of the left iliac vein between the overlying right common iliac artery and the fifth lumbar vertebrae. It is usually asymptomatic but may predispose to deep vein thrombosis (DVT), an important cause of pregnancy-related morbidity and mortality. In symptomatic patients, management may include pharmacological treatment (anticoagulants or coumarins), thrombectomy and, to avoid recurrence of DVT, stent placement.

\section{Case}

A 27-year-old woman, G2C1, was referred to our prenatal care due to previous DVT caused by MTS. Three years ago, she had a sudden pain in the region of buttocks that radiated to her left leg. During physical examination it was observed asymmetry between legs, swelling and increased left thigh circumference associated with local erythema. Abdominal CT scan demonstrated stenosis of the proximal segment of the left common iliac vein, between the right common iliac artery and the fifth lumbar vertebrae. Doppler ultrasound showed left common and external iliac veins without flow or compressibility. At that time, she was using combined oral contraceptives (drospirenone and ethinylestradiol). She had no personal or family history of deep venous thrombosis, acute myocardial infarction, stroke or smoking. She had no problem during her first pregnancy or postpartum. She was hospitalized for 20 days and treated with enoxaparin (1mg/ $\mathrm{kg}$ twice a day), and evolved with total improvement of symptoms. Oral contraceptives were suspended. She was discharged home using compression stockings, rivaroxaban, and coumarin/ troxerrutin for six months and remained asymptomatic since then. She chose intrauterine device as contraceptive method. Approximately two years later, she got pregnant. For thromboprophylaxis, it was recommended the use of compressive stockings, unfractionated heparin 10,000UI twice a day, and low dose aspirin (100mg/day). She had an uncomplicated pregnancy, with no adverse effects from the treatment. With 39 weeks of gestation, she was admitted to labor and delivery ward in active phase of labor. After several hours, a cesarean section was done due to first stage labor arrest. She gave birth to a healthy female baby, with 2,790g, Apgar score of 9 and 9. In the postpartum period, the patient remained in use of compression stockings and unfractionated heparin, and had a good outcome, without complications.

\section{Conclusion}

May-Thurner syndrome should always be remembered in young women with DVT, especially when it occurs in left leg. Due to hypercoagulable state and venous stasis, pregnancy represents an important risk factor for developing deep vein thrombosis and pulmonary embolism. Despite this fact, the reported case demonstrates that use of unfractionated heparin was effective to prevent a new episode of DVT during pregnancy and puerperium. 


\section{E1142 - TIME DURATION AND DIFFICULTY TO ASSEMBLE A SYSTEM TO CONTROL POSTPARTUM HAEMORRHAGE WITH CONDOM AND FOLEY BLADDER CATHETER}

Marrie Martins M. ${ }^{1}$, Knobel R. ${ }^{1}$, Volpato L. ${ }^{1}$, Koettker Silveira S. ${ }^{1}$, May Feuerschuette O. ${ }^{1}$, Pivetta Cantarelli M. ${ }^{1}$, Trapani $\operatorname{Jr}$ A. ${ }^{1}$

Hu/ufsc/ebserh/ Women, Gynecology and Obstetrics, Florianópolis-Brazil ${ }^{1}$

\section{Objective}

One of the problems with the use of condom and probe balloon uterine tamponade may be the time spent and the difficulty in assembling. The objective of this study was to evaluate the time duration and level of difficulty presented by health professionals in the assembly of an intra-uterine baloon with a Foley catheter and condom.

\section{Methods}

The present experimental study invited to participate medical students, physicians, obstetric nurses, midwifes and nurse technician.

During a course of obstetric emergencies and in a obstetric center of a universitary hospital, several professionals were instructed on methods to control postpartum haemorrhage. They were instructed on indications, contraindications, method of assembly, insertion, control and removal of the intrauterine balloon.

The participants were then asked to assemble intra-uterine baloon with a foley catheter number 22, placed in a condom without lubricant and tied with surgical thread. (figure 1). A self-administered questionnaire was used to collect data from the participants as well as to evaluate the difficulty of assembly. The time in seconds that was used to assemble the system was measured by the researchers. All mounted systems were tested (filled with $500 \mathrm{ml}$ of water) in in a puerperal uterus simulator to prove their functionality.

\section{Results}

A total of 49 (59.04\%) physicians or medical students, 25 (30.12\%) nurses / obstetrician nurses and 9 (10.84\%) nursing technicians participated in the study. Thereby were 83 simulations.

All participants were able to set up the system. There were significant leakages in 2 assemblies (2.4\%). The mean assembly time was 99.6 seconds, with a standard deviation of 57.8 seconds. There was no difference in assembly time according to the years of experience, and students had the same assembly time as professionals with more than 20 years of experience. Most participants considered assembly of the system very easy or easy (table 1)

\section{Conclusion}

With the available material and quick instructions all the professionals who participated in this study were able were able to make an intrauterine tamponade system. System mounting is fast (averaging 99 seconds) and does not vary with the professional experience. Most of the participants considered assembly of the system easy or very easy.

In conclusion, it would appear that the intrauterine system made with bladder catheter and condom is an alternative for control of postpartum hemorrhage cheap and effortless. 
E1252 - THE RELATIONSHIP BETWEEN USE OF NIFEDIPINE AND POSTPARTUM BLOOD LOSS IN PATIENTS WITH PRETERM LABOR

Kiyak H. ${ }^{1}$, Süzen Caypinar S. ${ }^{2}$, Acar Z. ${ }^{3}$, Atis Aydin A. ${ }^{2}$, Üstün B. ${ }^{4}$, Aytufan Z. ${ }^{4}$, Gedikbasi A. ${ }^{2}$

Istanbul Kanuni Sultan Suleyman Training and Research Hospital, Obgyn, Istanbul-Turkey ${ }^{1}$

Istanbul Kanuni Sultan Suleyman Education and Teaching Hospital, Maternal Fetal

Medicine, Istanbul-Turkey ${ }^{2}$

Istanbul Kanuni Sultan Suleyman Education and Teaching Hospital, Maternal Fetal Medicine, Istanbul-Turkey $^{3}$

Istanbul Kanuni Sultan Suleyman Education and Teaching Hospital, Obgyn, Istanbul-Turkey ${ }^{4}$

\section{Objective}

To determine whether exposure to nifedipine before delivery is associated with an increased risk of postpartum blood loss in patients with preterm labor

\section{Methods}

This was a retrospective study from 2012 to 2019 and a total of 486 patients who admitted due to preterm labor were included. Patients who were given nifedipine for tocolysis before delivery were considered as the study group (n: 240), and the patients who gave birth without getting tocolysis constituted the control group $(n=246)$. In addition to dose of nifedipine, used during the last 24 hours, 72 hours and 1 week before delivery, the total dose of nifedipine given and the duration from the last dose to the delivery were recorded separately. Hemoglobin and hematocrit values measured before and 6 hours after delivery were recorded and postpartum bleeding amount was calculated.

Results: No significant difference was observed in terms of mean difference between prepostpartum hemoglobin and hematocrit levels between control group and nifedipine group $(p=0.510),(p=0.411)$. However, in subgroup analyzes for time until delivery and withdrawal of nifedipine, a positive correlation was observed between difference in hemoglobin and hematocrit levels for pre- and postpartum levels and delivery within 24 hours after withdrawal of nifedipine ( $\mathrm{r}=0.176 \mathrm{p}=0.006),(\mathrm{r}=0.139 \mathrm{p}=0.030)$.

Conclusion: The use of nifedipine in patients with preterm labor and related tocolysis can increase the risk for postpartum blood loss only for cases that deliver within 24 hours after withdrawal. 


\section{E1271 - MANAGEMENT OF POSTPARTUM HEMORRHAGE DUE TO PLACENTARY CAUSE}

Otaño J. ${ }^{1}$, Estefanía A. ${ }^{1}$, Pedroarena I. ${ }^{1}$, Abian N. ${ }^{2}$

Complejo Hospitalario De Navarra, Obstetrics and Gynecology, Pamplona-Spain ${ }^{1}$

Hospital Reina Sofía, Obstetrics and Gynecology, Tudela-Spain ${ }^{2}$

Postpartum hemorrhage is classically defined as a blood loss of $>500 \mathrm{cc}$ after vaginal delivery, $>$ 1000cc after cesarean section or an hemorrhage that causes hemodynamic instability. Its causes can be classified in four groups known as the four ' $\mathrm{T}$ ': lack of uterine tone, trauma, coagulopathy (trombine) and tissue retention. It is a major cause of maternal morbidity and causes up to $4 \%$ of maternal death in developed countries, what makes it necessary to diagnose early and to give a quick treatment.

\section{Case}

A 34 year old patient, $37+1$ weeks pregnant with a previous cesarean section and with no medical history of interest. Adequate control of pregnancy, without superimposed pathology. The patient was admitted for labor induction with vaginal E2 prostaglandins due to an early rupture of membranes. As medication, she received antibiotic coverage for group $\mathrm{B}$ streptococcus and oxytocin. The evolution of dilatation was satisfactory and during the second stage of labour a Mälstrom vacuum was performed because of a lack of descent of the presentation. The placenta was delivered by Crede manoeuvre, resulting difficult and with a subjective moderate bleeding, reason why $1 \mathrm{~g}$ of intravenous tranexamic acid and $1000 \mathrm{~g}$ of rectal E1 prostaglandins were administered. At the examination, the placenta was bilobated and appeared complete.

After that, revision of the birth canal was performed, showing a II grade vaginal tear, which was sutured. During the suture, the patient started feeling dizzy and showed cutaneous paleness with a blood pressure of $62 / 35$. The uterus was contracted, so she was given gelafundin, oxygen and efedrin reaching a pressure of 80/45 and hemodynamic stability. After 50 minutes, the patient experienced a new worsen and showed uterine atony. At that point, a blood count and a cross-matching test were requested and the patient was moved to the operating room. Under antibiotic coverage with $2 g$ of cefazolin and sedation with midazolam plenty of clots were taken out and a placental cotyledon was removed from the right uterine horn, with subsequent administration of 20 units of oxytocin and uterine massage.

The blood count showed an haemoglobin of 7,6 g/dL so during the 4 days of hospitalization the patient was transfused 3 concentrates of red cells with an haemoglobin of $11 \mathrm{~g} / \mathrm{dL}$ at discharge.

10 days after delivery, the patient attended the emergency service due to a major bleeding. The uterus was contracted and the echography showed an hyperechogenic area at the anterior wall of the uterus, compatible with a subinvolution of the placental-site. A new blood count was performed, showing a $13 \mathrm{~g} / \mathrm{dL}$ haemoglobin. As the bleeding was continuous, a Foley catheter filled with 10cc was placed into the uterus in order to make haemostasis. After 2 days, the catheter was removed without incidences and the patient had a satisfactory evolution.

Postpartum hemorrhage may lead to a quick haemodinamic worsen of a patient and is a potential cause of morbidity and dead. For that reason, identifying its cause and giving a rapid treatment is crucial in order to avoid major complications. 


\section{E1349 - THE EFFICACY OF PARENTERAL IRON ISOMALTOSIDE 500 (MONOFER) THERAPY IN WOMEN WITH POSTPARTUM IRON DEFICIENCY ANEMIA}

Berovic M. ${ }^{1}$, Gall V. ${ }^{1}$, Sabolovic Rudman S. ${ }^{1}, \underline{\text { Kosec V. }}{ }^{1}$

University Hospital Center Sestre Milosrdnice, Gynecology and Obstetrics, Zagreb-Croatia ${ }^{1}$

\section{Objective}

Our goal was evaluation of Monofer efficacy on correcting severe postpartum anemia (based on measured haemathological parameters and clinical symptoms such as pallor, dyspnoea, fatigue, presyncope), and its effect on hemoglobin (Hb) levels checked up after 6 weeks. Accordingly, if intravenous iron therapy can replace blood transfusions for relieving clinical symptoms of severe anemia due to iron deficiency in postpartum period.

\section{Methods}

Single-center, open-label trial, uncontrolled study

Participants were 32 women who had given birth, regardless of vaginal delivery or Cesarean section, and were monitored in the period between November 2018 to May 2019. The total of 32 women had hemoglobin concentrations in early postpartum period (first to fourth day after the labor) between 67 and $84 \mathrm{mg} / \mathrm{l}$, with more or less expressed clinical symptoms, thus have been candidates for intravenous iron therapy with iron isomaltoside 500 to correct postpartum iron deficiency anemia (IDA) . The primary outcome was estimation of the increase of $\mathrm{Hb}$ concentrations 6 weeks after the Monofer injection. Women were advised to continue with per oral iron substitution if needed a month after the injection of Monofer.

\section{Results}

We found clinically significant improvement of fatigue and other symptoms, as well as in hematological parameters after the treatment with Monofer. Mean preinfusion Hb was found to be $75,25 \mathrm{~g} / \mathrm{l}$ and mean postinfusion $\mathrm{Hb}$ was $115 \mathrm{~g} / \mathrm{L}$.

\section{Conclusion}

Iron isomaltoside 500 (Monofer) has demonstrated clinically significant efficacy in women with postpartum iron deficiency anemia therefore could be the first choice therapy for correcting IDA when in doubt about whether to give blood transfusion or not. Consequently, there is less need for blood transfusion, with minimal risk of anaphylactic reaction, faster recovery and shorter hospitalization due to Monofer substitution therapy therefore providing more cost-effective practice. 
E1372 - GOOD END RESULTS IN SPITE OF MEDICAL MISTAKES

Ruiz Garcia M. ${ }^{1}$, Bazán Legasa M. ${ }^{1}$, Estefanía Díaz A. ${ }^{1}$, Otaño Ruiz J. ${ }^{1}$,

Zabaleta Loinaz I. ${ }^{1}$, Abián Franco N. ${ }^{2}$

Complejo Hospitalario De Navarra, Gynecologist, Pamplona-Spain ${ }^{1}$

Hospital Reina Sofía, Medical Resident, Tudela-Spain ${ }^{2}$

Placenta previa refers to the presence of placental tissue that extends over the internal cervical ostium. The management of pregnancies complicated by placenta previa is best addressed in terms of the patient's clinical setting, concerning asymptomatic or actively bleeding women. The province wide incidence of complications due to placenta previa may be high. Regarding maternal morbidity we consider antepartum bleeding, postpartum hemorrhage, maternal anemia, comorbid adherent placentation and hysterectomy. Concerning neonatal complications we find preterm birth, low birth weight less than $2500 \mathrm{~g}$, Apgar-score after five minutes less tan 7 , and fetal mortality.

We present two cases of non diagnosed placenta previa which fortunately finished at good outcome.

The first one was a patient ongoing her first pregnancy with no complications described at the moment. An external cephalic version was scheduled as breech presentation remained by week 37 of gestation. Vaginal bleeding was objectified while the maneuvers were performanced, when occlusive placenta previa was diagnosed by echography (not described before). Inmediately an urgent cesarean section was indicated and a female neonate of 2465 grams was born with Apgar-score of 8-9-10 (1'-5'-10')and $\mathrm{pH}$ of 7'28-7'41 (arterial and venous respectively)

The second case involved a multiparous woman. She was on her sixth pregnancy and had the following obstetric history: three normal vaginal deliveries, one miscarriage which required obstetric curettage and one cesarean section due to spontaneous rupture membrane and breech presentation. She arrived at the emergency room at week 36 because of severe vaginal bleeding, uterine contractions and feeling dizzy. During exploration, it was made a vaginal exploration appreciating a cervix dilatation of $5 \mathrm{~cm}$ and touching the placenta but not the baby presentation. Two days before she had consulted by uterine contractions and very scarce bleeding without diagnosed placenta previa. An urgent cesarean section was performance due to the diagnose of previa placenta and a hypovolemic shock. A male neonate of 2960 was born with Apgar-score of 4-6-8(1'-5'-10') and arterial/venous $\mathrm{pH}$ of 7'15-7'18. This patient requires the transfusion of five red blood cells concentrates because of being unstable hemodynamically with and hemoglobine of $5.8 \mathrm{~g} / \mathrm{dl}$.

These two are examples which could have finished in very poor outcomes due to an initial wrong medical diagnose.

Placenta previa increases the risk of morbidity and mortality both maternal and fetal. The diagnostic suspicion based on risk factors and echocardiography signs are essential to anticipate possible complications that may arise. 


\section{E1379 - IS RADICAL SURGERY NECESSARY FOR PLACENTAL IMPLANTATION ABNORMALITIES (PIAS) (YALINKAYA'S TECHNIQUE)}

Yalinkaya A. $^{1}$, Elci E. ${ }^{1}$, Bozbay N. ${ }^{1}$

Dicle University Medical Faculty, Obstetrics and Gynecology, Diyarbakir-Turkey ${ }^{1}$

\section{Objective}

The aim of this study was to describe a new comprehensive and complete conservative surgical technique (developed by Yalinkaya) in all form of PIAs for preserving the patient's future fertility. Therefore, we wanted to discuss our experience to contribute to the literature.

\section{Methods}

We prospectively designed this study which includes 152 patients between June 2013 and October 2018. The data were collected from hospital and special recorded patient's files. Most of the patients were referred by different centers and all of them were examined by a perinatologist with transvaginal ultrasound to confirm PIAs. The patients' demographics and medical characteristics were recorded. Antenatal and perinatal PIAs risks, delivery time and place were discussed with them and their relatives. The patients All of were operated by an experienced surgeon (Yalinkaya) with his surgical technique. Used surgical technique and technical details, blood transfusion, maternal complications and mean duration of hospitalization were assessed.

\section{Results}

The patients placenta previa were had with PIAs at least one or more previous cesarean section (CS).The mean maternal age, gestational age, infant weight and repeated cesarean section were found in 33.21+4.70 (22-44) years, 35.46 \pm 4.99 (11-39) weeks, 2624 \pm 836 (304175) gram and $3.42 \pm 1.03$ (2-6) times, respectively. The operations were performed in 60 (39.47\%) patients before the planned periods due to their emergency. Spontaneous intraabdominal hemorrhage was observed in 5 (3.28\%) pregnancy and bladder bleeding was observed in $1(0.65 \%)$ of them.

The operation was performed to 143 (94.07\%) patients under spinal anesthesia and performed to $9(5.92 \%)$ patients under general anesthesia due to medical indications. All of the patients were operated as a normal cesarean section by using Pfannenstiel (Kerr) incision for abdominal wall and lower uterine segment transverse incision for uterine wall. The surgical complications were occurred in 4 (2.63\%) cases during entering to abdominal cavity and minimal bladder wall injury was occurred, and repaired with primary suture by the surgeon with no urologist. Vesico-uterine fistula was developed in one patient 4 to 5 months later as a late complication, and repaired via laparotomy by the same surgeon. The average preoperative and postoperative hemoglobin $(\mathrm{Hb})$ and hematocrit $(\mathrm{Htc})$ levels were calculated. A total 81 units RBC were transfused to 34 patients who required blood transfusions. Hysterectomy and vessels ligations were not performed to all cases. The patients did not need intensive care unit (ICU), except three of them with other pregnancy complications. The mean duration of hospitalization was $1.92 \pm 1.34$ days, and maternal mortality did not occur in any cases.

\section{Conclusion}

There are limited data available for optimal management of PIAs in the literature until now. If Yalinkaya technique can be used by experienced surgeons for PIAs, it is the most effective among all of other methods so far, and strongly preserves the patient's life and future fertility, and also leads to reduce the hospital costs and maternal morbidity and mortality. Therefore, we think that this is the most effective technique for PIAs patients. 


\section{E1406 - POSTPARTUM HAEMORRHAGE CAUSED BY PLACENTA ACCRETA INCRETA}

$\underline{\text { Resic Karara J. }}{ }^{1}$, Delić B. ${ }^{1}$, Vulić M. ${ }^{1}$, Kosović I. ${ }^{1}$, Viđak J. ${ }^{1}$, Kopić D. ${ }^{1}$ Clinical Hospital Split, Clinic of Obstetrics and Gynecology, Split-Croatia ${ }^{1}$

The incidence of placenta accreta, increta has increased. It is now one of the common causes of postpartum haemorrhage and consequent maternal morbidity.

This paper describes case of a woman, 48 years old, twin pregnancy from donated oocyte. She was hospitalized in the 31st gestational week because of cholestasis and gestational diabetes. The Cesarean section was done because of obstetrical history. Postoperative period was normal, but on the 6th day after C-section she experienced massive uterine haemorrhage. Ultrasound examination showed enlarged uterus with dilated cavum uteri with hyperechogenity, which measured $6 \times 8 \mathrm{~cm}$. The patient was proceeded to the operating room to do curettage: $2 \times 0,25 \mathrm{mg}$ of misoprostol was given intracervical but uterus didn `t contract. She got extreme bradycardia and extreme hypotensive crisis 15-20 minutes, after getting second ampulla of misoprostol. After that they tried Bakri Balloon Tamponade. As the bleeding didn`t stop, hysterectomy was done. Cause of haemorrhage had been placenta accreta and increta with massive occlusion with trophoblast. On the 15th postoperative day the patient went home.

\section{Conclusion}

Placenta accreta and increta is responsible for postpartum haemorrhage and often is associated with hysterectomy and maternal morbidity. 


\section{E1455 - POSTPARTUM FEMALE SEXUAL PROBLEMS AND RELATED CONDITIONS IN HUNGARY A CROSS SECTIONAL STUDY \\ Szöllosi K. ${ }^{1}$, Szabó L. ${ }^{1}$}

Semmelweis University, Department of Family Care and Methodology, Budapest-Hungary ${ }^{1}$

\section{Objective}

However female sexual dysfunctions are common among women in the postpartum period, the profile of these disturbances has not been well investigated in Hungary yet. The aim of the study was to evaluate the postpartum female sexual functions in Hungary. This research sought to investigate the possible predictor factors which can influence postpartum female sexual functions.

\section{Methods}

This was a cross-sectional study including patients from two maternity clinics in Budapest. 113 women were recruited into our study 3 months after their childbirth. 53 had vaginal birth, 60 had caesarian section. Data was collected from medical reports and by using selfdeveloped questions and validated questionnaires in order to measure five important predictors which may be responsible for postpartum sexual dysfunctions such as (1) mode of delivery, (2) parity, (3) urinary incontinence and (4) body image. Sexual functions were evaluated by the Hungarian version of Female Sexual Function Index (FSFI). The Hungarian version of Body Image Questionnaire-Short Form14 (BSQ-SF14) was applied for assessing body image.

\section{Results}

$82,3 \%$ of the participants began to have sexual intercourse within three months postpartum. $53,98 \%$ of the participants reported sexual dysfunctions (cut-off FSFI score 26,55). According to our results mode of delivery, parity, hemorrhoids, time of intercourse resumption were not associated with female sexual dysfunctions. We found correlation at a tendential level between urinary incontinence and sexual dysfunctions $(p=0,003, R=0,26)$. We found negativ correlation at a tendential level between the total score of BSQ-SF14 and FSFI ( $\mathrm{p}=0,03, \mathrm{R}=-$ 0,269 ). Only $32,74 \%$ of women reported discussing sexual life with health care professionals, however $67,25 \%$ of them would have had the need to be asked about their postpartum health issues.

\section{Conclusion}

The prevalence of female sexual dysfunctions was relatively high after childbirth. We found that incontinence and body image were associated with sexual dysfunctions, other risk factors remained unknown. Despite regular contact with health care professionals, women rarely get any information about postpartum sexual health issues. The high prevalence of dysfunctions indicate the need for further investigation to address other risk factors and proper counselling of women after childbirth. 


\section{E1470 - THE OUTCOMES OF MANAGEMENT OF VAGINAL DELIVERY AFTER CESAREAN SECTION}

Stavinskaia L. ${ }^{1}$, Tabuica U. ${ }^{1}$, Sarbu Z. ${ }^{1}$, Petrov V. ${ }^{2}$, Mamoncic E. ${ }^{2}$

State University of Medicine and Pharmacy "Nicolae Testemitanu", Department of

Obstetrics and Gynecology, Chisinau-Moldova ${ }^{1}$

Institute of Mother and Child, Department of Obstetrics and Gynecology, Chisinau-Moldova ${ }^{2}$

\section{Objective}

Currently, the issue of increasing rate of cesarean section (CS) is a serious concern either among professionals and society. Country statistics show a stable growing trend. On average, 1 from 5 pregnant women in the world have CS. According to the data of the Institute of Mother and Child (the only third level maternity hospital in the Republic of Moldova) in 2015 the rate of CS was 33.6\%, in $2016-30.5 \%$, in 2017-30.7\%, in $2018-31,8 \%$. The main indication for repeated CS is the scar on the uterus after the previous or myomectomy. It is well known that each subsequent surgery aggravates the prognosis and increases the number of complications. For these reasons, a solution in reducing the above-mentioned complications is to reduce the cesarean rate by vaginal delivery in the possible cases.

\section{Methods}

A retrospective study of 2316 patients who had a history of scar on the uterus after a CS was performed in the obstetrics departments of the IMC between 2015 and 2017. Over 3-years period, with the general decreasing of births at the IMC (2015- 6493 cases, 2017-6122 cases) and across the country, there has been a steady increasing number of women with uterine scarring after previous CS, namely 808 (13.2\%) in 2017 vs 756 (11.3\%) in 2015. It is important to note that the IMC is the only third level institution in RM where are admitted the maximum number of women with this pathology, including those with 3 or more interventions. This leads to a regular grow of the number of repeated CS, from $31.22 \%$ (2014) to $37.8 \%$ (2017).

\section{Results}

The implementation of the national protocol for vaginal delivery after previous CS in January 2015 allowed to decrease the rate of CS from 33.6\% in 2014 to $30.7 \%$ in 2017. The number of vaginal births after previous abdominal birth grew from 7.7\% (2014) to 12.1\% (2017) of women with scarring on the uterus. From 406 patients selected for vaginal birth, $76.3 \%$ had natural delivery, $23,7 \%$ of cases were finished through repeated CS. Only 38.96\% had at least one vaginal birth that proves the patient's good selection. In $21.25 \%$ of vaginal delivery cases, the labor was completed by vacuum-extractor assistance because of acute fetal hypoxia, prolonged second stage of the labor. Stimulation of labor was required in $12.5 \%$ of births. There were no pathological haemorrhage during the third period of labor. Regarding to the perinatal results only in two cases children were born with 4/5 points Apgar score due to acute hypoxia, the rest having $7 / 8$ points

\section{Conclusion}

The rational management of women with scarring on the uterus allow to reduce the risk of obstetrical and perinatal complications. In the case of mature pregnancy and biological matured cervix in patients with scarring on the uterus, it is possible to induce labor through early amniotomy. The degree of maturity of the cervix and the spontaneous onset of labor is a preliminary factor indicating the competence of the scar on the uterus. 


\section{Obstetrics - Preterm Premature Rupture of Membranes}

\section{E1147 - E.COLI GENITAL INFECTIONS IN WOMEN WITH PRETERM PREMATURE RUPTURE OF MEMBRANE AND PREGNANCY OUTCOMES RETROSPECTIVE STUDY}

$\underline{\operatorname{Copos} A .^{1}}$, Cuc R. ${ }^{2}$, Mascas G. ${ }^{2}$, Aur C. ${ }^{1}$, Negrini R. ${ }^{1}$, Mocuta D. ${ }^{2}$

Spitalul Clinic Judetean De Urgenta Oradea, Obstetrics-Gynecology, Oradea-Romania ${ }^{1}$

Spitalul Clinic De Urgenta Oradea, Obstetrics-Gynecology, Oradea-Romania ${ }^{2}$

Premature rupture of membranes (pprom) refers to a patient who is beyond 37 weeks 'gestation and has presented with rupture of membranes (rom) prior to the onset of labor and is associated with high fetal and maternal risk. Vaginal bacterial colonization is one of the most important etiologies in pprom.

\section{Objective}

This study was designed to highlight genital infections in preterm delivery with prom and pregnancy outcome.

\section{Methods}

Our retrospective and randomized trial(study)consist of 277 pregnant woman with preterm delivery(24-27 weeks of gestation) who were assisted at birth in the last 2 years( 1 january 2017- 31 december 2018), in an academic hospital of Oradea University- department of medical science. 166 of the cases were with prom. The results were obtained based on routine vaginal secretion cultures.

\section{Results}

In our department, in last 2 years the incidence of premature delivery was 3,8\% (277 cases) out of a total of 7194 births. Also, 41,87\% of pregnant woman with premature labor were with pprom. Is put in evidence that almost half of the cases $(43,10 \%)$ were found to have genital infections. Most isolated pathogens from uro-genital cultures was escherichia coli (72\%), followed by gardnerella (6\%), coagulase-negative staphylococci (4\%), proteus vulgaris (4\%) and other microorganisms with lower frequency (proteus mirabilis, staphylococcus aureus, enterobacter, trichomonas vaginalis). 15,3\% of genital infections were also associated with candida albicans. The study does not reveal a higher risk of chorioamnionits (only 8\%) or congenital pneumonia.

\section{Conclusion}

Based on results of this study, vaginal cultures are essential in early diagnosis and management of genital infections (most frequent with e.coli) associated with preterm delivery in order to decrease fetal and maternal complication. 
E1255 - PREVIABLE PRETERM MEMBRANE RUPTURE MATERNAL AND FETAL OUTCOME

Kiyak H. ${ }^{1}$, Güneş Gülcan A. ${ }^{1}$, Özcan Yüksel S. ${ }^{1}$, Gedikbasi A. ${ }^{1}$

Istanbul Kanuni Sultan Süleyman Tranining and Research Hospital, Obgyn, Istanbul-Turkey ${ }^{1}$

\section{Objective}

The aim of this study is to evaluate maternal, neonatal, fetal outcomes of pregnant patients diagnosed with preterm premature membranous rupture before 24 weeks with follow-up until birth and related factors affecting outcome.

\section{Methods}

This retrospective study was planned in screening patients applied to our clinic with confirmed preterm membrane rupture before the $24+0$ gestational weeks, diagnosed between April 2012 and August 2017. Inclusion criteria were patients with singleton gestations between 14-24 gestational weeks and confirmed diagnosis of PPROM. Gestations complicated with a fetal anomaly, fetal death during application, families opting for termination of pregnancy and cases with multiple gestations were not included in the study. We recorded demographic characteristics, medical history, gestational age at diagnoses of PPROM, risk factors, parameters during follow-up and management, related data of delivery, maternal and neonatal complications in the postnatal period of the patients in our study.

\section{Results}

The study included 192 patients, of whom in 21 (10,94\%) patients intrauterine fetal death occurred. 67 (34,8\%) of newborns were lost during follow-up in intensive care unit after delivery. Finally, 104 (54,16\%) of these infants were discharged alive. We compared in two groups alive $(n=104)$ and dead $(n=67)$ patients medical data. Statistically significant variables in multivariate regression analysis affecting neonatal survival are amnion fluid volume, the presence of oligohydramnios and / or anhydramnios, duration of PPROM, average of first minute Apgar, average of neonatal birth weight. ROC analysis showed us that gestational week at birth was determinant for fetal death prediction.

\section{Conclusion}

$57.14 \%$ of patients resulted with neonatal survival with diagnoses of PPROM before 24+0 gestational weeks. The most important determinants in these cases for survival were duration of PPROM, gestational age at birth and amount of amniotic fluid. 
E1428 - GESTATIONAL TROFOBLASTIC DISEASE MOLA PARTIAL TYPE AND BORN LIVE

Capuz F. ${ }^{1}, \underline{\text { Loza H. }}{ }^{1}$

Axxis Hospital, Gynecology, Quito-Ecuador ${ }^{1}$

\section{Case}

26-year-old patient, G 2 A 1. First control, 14-week pregnancy. First ultrasound says placenta with signs of partial molar type hydropathic degeneration. Laboratory Tests normal, BHCG $87219 \mathrm{mIU} / \mathrm{ml}$

Amniocentesis in week 18, karyotype in amniotic fluid: 46 XX.

Monthly prenatal check-ups are performed without complications, but at 29 weeks it presents PREPROM, 140/90 blood pressure, 24-hour urine protein: $332 \mathrm{mg} / 24 \mathrm{~h}$. Ultrasound: live single fetus, anhydramnios, weight $1200 \mathrm{~g}$. Diagnosis: Pregnancy of 29 weeks + PREPROM + Preeclampsia + Gestational trophoblastic disease Partial mola type.

Plan: cesarean.

Is obtained newborn female living weight $1105 \mathrm{~g}$, large posterior placenta with partial mole characteristics?

Pathology: weight $1600 \mathrm{~g}$, cord $19 \mathrm{~cm}$, spongy appearance and areas with vesicles. Double population of irregular chorionic villi: fibrotic and hydropathic. RX chest: normal. Puerperio: BHCG, $24 \mathrm{~h}(3815 \mathrm{mIU} / \mathrm{ml}), 1$ week $(2520 \mathrm{mIU} / \mathrm{ml}), 1$ month $(0.68 \mathrm{mIU} / \mathrm{ml})$ and the year $(<0.05 \mathrm{mIU} / \mathrm{ml})$.

Definition: It is defined as a rare complication of pregnancy characterized by abnormal proliferation of trophoblastic tissue. It consists of an abnormal pregnancy characterized by hydropic degeneration of the chorionic villi and trophoblastic proliferation with abnormal or absent embryonic development.

Etiology: Polyspermia etiology: is the most frequent, the oocyte is fertilized by 2 sperm (twins)

Oocyte fertilized by a diploid sperm, due to a defect in the first meiotic division. Very infrequent

Clinical Presentation: Asymptomatic cases 41\%. Hemorrhage is present in $72 \%$ of patients. Uterine height greater than expected by gestational age (3.7\%). Preeclampsia (2.5\%). Low association with hyperthyroidism, hyperemesis gravidarum and luteal teak ovarian cysts (2\%). This entity has a benign behavior in most cases and the risk of malignant transformation is around $4 \%$. 
E1466 - THE EFFECTS OF CONTINUOUS AMNIOINFUSION ON FETAL SURVIVAL IN PRETERM PREMATURE RUPTURE OF MEMBRANE

Yalinkaya A. $^{1}$, Bozbay N. ${ }^{1}$, Elci E. ${ }^{1}$

Dicle University Medical Faculty, Obstetrics and Gynecology, Diyarbakir-Turkey ${ }^{1}$

\section{Objective}

Deficiency or absence of amniotic fluid due to preterm premature rupture of membrane (PPROM) may cause various complications such as limitation of movement of the fetus, growth retardation, failure of lung development and cord compression from intrauterine asphyxia to fetal death. Therefore, by increasing the amniotic fluid with continuous amnioinfusion, it is tried to prolong the pregnancy. The aim of this study was to present the effects of continuous amnioinfusion on continuation of pregnancy in order to treat oligohydramnios caused by preterm premature rupture of membrane.

\section{Methods}

This study was planned retrospectively between January 2014 and December 2018 in our clinic. Demographic and clinical data of all patients were obtained from hospital records, and the latest information of patients was also obtained by phone. Continuous amnioinfusion was performed to 13 of 15 PPROM patients and two patients were excluded due to spontaneous increase in amniotic fluid. In addition, the last data of an $800 \mathrm{~g}$ baby who underwent continuous amnioinfusion and was born alive after 8 days was not reached. All patients who underwent continuous amnioinfusion were examined by a perinatologist and treated with antibiotics because of preterm premature rupture of membrane. Continuous amnioinfusion was performed to only PPROM cases by using an epidural catheter. On the other hand, the cases that had vaginal bleeding, uterine contractions, symptomatic infections, and oligohydramnios with intact membrane were excluded. All patients were informed about possible risks of the procedure, and informed consent was obtained from the patients.

\section{Results}

Continuous amnioinfusion was planned for 15 cases of PPROM, but performed in 13 cases. The mean age of the patients was $31.15 \pm 4.37$ (24-42), gravida 2.76 \pm 1.48 (1-5) and parity $1.38 \pm 1.44$ (1-4). At the inception of continuous amnioinfusion, the mean gestational week was $22.07 \pm 1.93$ (19-25) and the amniotic fluid index 30.00 \pm 11.54 (15-50) mm. However, the mean gestational age at delivery was $26.38 \pm 3.73$ (20-34) weeks. Gestational age was prolonged on average 4.31 weeks by performing continuous amnioinfusion. The average of prolonging time from the procedure to the delivery was found $30.53 \pm 24.42(2-84)$ days. 5 (38.46\%) of patients were delivered vaginally and 8 (61.53\%) were delivered by cesarean section. Among 13 patients who underwent continuous amnioinfusion, 9 (69.23\%) of the fetuses were born alive. 6 (45.15\%) of them are surviving their life, but 1 (7.69\%) of them is unknown. However, the remaining 6 (46.15\%) cases resulted in death, of these, 4 (30.76\%) intrauterine mortality due to unknown reasons and 2 (15.38\%) died after delivery in the early perinatal period. All live-born infants were treated in the neonatal intensive care unit.

\section{Conclusion}

In this study, 46.15\% of PPROM patients were survived with continuous amnioinfusion and the mean gestational period was prolonged in 4.23 weeks. There are not enough studies so we need many more comprehensive studies. We suggest that, continuous amnioinfusion has positive effect on prognosis of PPROM cases. Therefore, it can be preferred in appropriate patients. 


\title{
Obstetrics - Fetal neurosonography and CNS anomalies
}

\author{
E1146 - FETAL INTRACRANIAL TERATOMA
}

Nascimento Pacheco E. ${ }^{1}$, Portella Martins A. ${ }^{1}$, Ferreira Cordeiro B. ${ }^{1}$, Coutinho Cavalieri J. ${ }^{1}$, Noya Galluzzo R. ${ }^{1}$, Souza Da Correggio K. ${ }^{1}$, Franco M. ${ }^{1}$, Koettker Silveira S. ${ }^{1}$, May Feuerschuette O. ${ }^{1}$, Rossi dos Santos G. ${ }^{1}$, Trapani Jr. A. ${ }^{1}$ Hu/ufsc/ebserh/ Women, Gynecology and Obstetrics, Florianópolis-Brazil ${ }^{1}$

Fetal intracranial tumors are rare representing 0.5 - 1.9\% of all fetal tumors. Among them, the most common histologic type is teratoma, composed by tissues from three germ layers, usually located at supratentorial level. The diagnosis may be suspected by ultrasound (US) of the second and third trimester. Teratoma appears as a complex heterogeneous mass, with solid and cystic components, and areas of calcification. Growth is rapid causing enlarged head circumference and distortion of brain anatomy. It may be accompanied by hydrocephalus and polyhydramnios. Definitive diagnosis can only be made after surgery. Since there is no intrauterine treatment available, the prognosis is usually lethal. Treatment of postnatal immature teratomas is controversial. They have a poor prognosis, with a low survival rate and a high percentage of psychomotor development delay and intracranial hypertension among survivors. The risk of recurrence in future pregnancies is low, since there is no association with family history or specific chromosomal abnormalities.

A 31-year-old pregnant woman, G2C1, Caucasian, was referred to our prenatal care due to fetal central nervous system anomalies evidenced by US scan performed at $34+2$ weeks of gestation. The US demonstrated an expansive lesion located in the left cerebral hemisphere, at choroid plexus topography, measuring 6.7 x $3.6 \mathrm{~cm}$, with low color Doppler vascularization, associated to macrocrania (biparietal diameter of $122.2 \mathrm{~mm}$ ), ventriculomegaly, and polyhydramnios (amniotic fluid index of $29 \mathrm{~cm}$ ). Subsequently, a magnetic resonance imaging was performed, which showed a brain tumor involving the left hemisphere suggestive of teratoma. At 35+6 weeks of gestation, she sought obstetric emergency complaining of sparse contractions and decreased fetal movement. At obstetrical examination, fetal heartbeat was undetectable. Fetal death was confirmed by US evaluation. Due to cephalopelvic disproportion, a caesarean section was performed. Fetus and placenta were submitted to histopathological analysis. Placental examination revealed scattered villous infarcts. Umbilical cord and fetal membrane had no relevant histological alterations. Visceral autolysis was demonstrated in a macerated, macrocephalic fetus, with a gestational age estimated between 35 and 36 weeks. Unfortunately, liquefaction of brain structures did not allow the identification of tumor or neoplastic process. 


\section{E1322 - ALOBAR HOLOPROSENCEPHALY CASE REPORT}

Kıvrak A. ${ }^{1}$, Karataş Y. ${ }^{2}$, Kalkan E. ${ }^{2}$, Çimen D. ${ }^{3}$, Sphaillari E. ${ }^{4}$, Sekhniashvili L. ${ }^{4}, \underline{\text { Örs } R .{ }^{4}}$ Konya Medova Hospital, Radiology, Konya-Turkey ${ }^{1}$

Konya Medova Hospital, Neurosurgery, Konya-Turkey ${ }^{2}$

Konya Medova Hospital, Pediatric Cardiology, Konya-Turkey ${ }^{3}$

Konya Medova Hospital, Neonatology, Konya-Turkey ${ }^{4}$

Holoprosencephaly is a serious central nervous system malformation that occurs as a result of failure to completely separate the cerebral hemispheres into two lobes. The disease is divided into three groups as alobar, semilobar and lobar type. Here, a case of alobar holoprosencephaly with ventriculoperitoneal shunt is presented because of its rarity. Case: Twenty-nine-year-old mother was born as 2090 grams after 30 weeks of gestation by cesarean section as the second child of her third pregnancy. After the tests, she was diagnosed as alobar holoprosencephaly. Echocardiography showed no anomaly except VSD and secundum ASD. Chromosomal test was normal. In brain ultrasonography; brain midline structures (3rd ventricle, corpus callosum, basal ganglia and interhemispheric fissure and falks) were not observed. Dilated mono ventricle was observed. Although the cortex was observed in the frontal region, no falx, parietal, temporal and occipital lobes were observed. Mesencephalon and pons were observed. The thalamus have a fusible appearance. The findings were consistent with alobar holoprosencephaly. Brain MRI The midline structures of the brain (3rd ventricle, corpus callosum, basal ganglia, and interhemispheric fissure and falx) were not observed. Dilated mono ventricle was observed. Although the cortex was observed in the frontal region, no falx, parietal, temporal and occipital lobes were observed. Mesencephalon and pons were observed. The thalamus has a fusible appearance. The findings were consistent with alobar holoprosencephaly. During follow-up, ventriculoperitoneal shunt was inserted with increased head circumference.

As a result, it was observed that the quality of life of the baby and his family improved with the insertion of a shunt in a baby with severe brain anomaly. We think that it is important to consider the quality of life in infants with severe anomalies except prognostic outcome. 
E1323 - OSTEOGENESIS IMPERFECTA TYPE III IN A NEWBORN CASE REPORT Örs R. ${ }^{1}$, Eklioğlu B. ${ }^{2}$, Atabek M. ${ }^{2}$, Spahillari E. ${ }^{1}$, Sekhniashvili L. ${ }^{1}$, Kıvrak A. ${ }^{3}$

Konya Medova Hospital, Neonatology, Konya-Turkey ${ }^{1}$

Ne.U. Meram Medical Faculty, Pediatric Endocrinology, Konya-Turkey ${ }^{2}$

Konya Medova Hospital, Radiology, Konya Turkey ${ }^{3}$

\section{Introduction}

Osteogenesis Imperfecta presents with recurrent bone fractures, bone deformities, hearing loss and blue sclera. The clinical picture and course of the disease vary widely among the different types of the disease. Here, it is reported that there are different molecular features in patients with similar clinical findings, as well as cases with similar phenotypic features in the same family, despite the genetic similarity in the family. Osteogenesis Imperfecta In most cases of Type 3, the transition is autosomal dominant and new mutations may occur. Type 3 Osteogenesis Imperfecta cases may present with multiple fractures and related deformities, or they may arise normally. In our case, there were relatively macrocephaly, triangular facial appearance, blue sclera and multiple fractures on the extremities, and these findings were consistent with Osteogenesis Imperfecta type 3.

\section{Case}

The fourth child of a 38-year-old mother from her fourth pregnancy was born by cesarean section at 39 weeks of age and weighing 2320 grams. There was no history of metabolic bone disease in the family. The diagnosis was confirmed by direct radiographs. When the patient was stable after early neonatal sepsis treatment, vitamin D and pamidronate from $1 \mathrm{mg} / \mathrm{kg}$ were planned for three days and every four months. Hypocalcemia developed and treated after pamidronate treatment. He was discharged after making recommendations to the family.

\section{Conclusion}

Severe forms such as Osteogenesis Imperfecta Type 3 can be recognized by intrauterine ultrasonography showing fractures. Our case is exceptional because the mother does not have a history of advanced age, fourth pregnancy and before, no family history, and antenatal diagnosis in the late period. However, genetic counseling may be given for subsequent pregnancies. Molecular analysis can be performed from chorionic villus during antenatal period and prenatal diagnosis can be made by chorionic villus biopsy in the first trimester of pregnancy in families with a history. 
E1324 - INTERHEMISPHERIC CYST ASSOCIATED WITH AGENESIS OF CORPUS CALLOSUM CASE REPORT

Karataş Y. ${ }^{1}$, Kaya B. ${ }^{1}$, Kalkan E. ${ }^{1}$, Kıvrak A. ${ }^{2}$, Sekhniashvili L. ${ }^{3}$, Spahillari E. ${ }^{3}, \underline{\text { Örs } R .}{ }^{3}$

Konya Medova Hospital, Neurosurgery, Konya-Turkey ${ }^{1}$

Konya Medova Hospital, Radiology, Konya-Turkey ${ }^{2}$ Konya Medova Hospital, Neonatology, Konya-Turkey ${ }^{3}$

\section{Introduction}

Congenital interhemispheric arachnoid cysts occur as a result of deviations in cerebrospinal fluid (CSF) flow during the early embryonic life of the primitive arachnoid membrane. The gap between the outer layer of the mesenchyme that will form the dura and arachnoid and the inner layer of the pia begins to fill with CSF from the 15th week. Herein we present a newborn with severe hydrocephalus diagnosed in antenatal period.

\section{Case}

The 36-year-old mother was born with a 36-week cesarean section as the second child of the second pregnancy. The parents were cousins. She was followed up due to prematurity and severe hydrocephalus. Physical examination was unremarkable. Eye, TORCH serology and chromosomal analysis were normal. Brain MRI showed an isointense cystic formation with 16x10x12 mm CSF filling the anterior cranial fossa from the level of the interhemispheric fissure and extending from the left cerebellar hemisphere adjacent to the posterior fossa. No dilatation was detected in the 4th ventricle. Enlargement of the right lateral ventricle is seen. It is thought to be associated with left lateral ventricular cyst and could not be selected separately. No dilatation was detected in the lateral ventricular frontal horns. Corpus callosum is not observed. The component of the cyst extending into the posterior fossa forms compression from the extraaxial distance to the left cerebellar hemisphere and the 4th ventricle. Ventriculoperitoneal shunt was inserted during follow-up. After the shunt infection, the shunt was removed. In the sixth month, the shunt was re-inserted. No problems were detected in the control.

\section{Conclusion}

The most appropriate treatment for interhemispheric arachnoid cysts is direct cyst fenestration or shunting of the cyst. The success rate of shunt surgery in interhemispheric arachnoid cysts is reported to be very high. 
E1325 - ANTENATAL BILATERAL SYMMETRICAL HEMORRHAGE IN THE BILATERAL TEMPORAL LOBE A CASE OF ALLLOIMMUNE AUTOIMMUNE NEONATAL THROMBOCYTOPENIA

Kıvrak A. ${ }^{1}$, Güven A. ${ }^{2}$, Tokgöz H. ${ }^{3}, \underline{\text { Ör } R .} .^{4}$

Konya Medova Hospital, Radiology, Konya-Turkey ${ }^{1}$

Ne. U. Meram Medical Faculty, Pediatric Neurology, Konya-Turkey ${ }^{2}$

Ne. U. Meram Medical Faculty, Pediatric Hematology, Konya-Turkey ${ }^{3}$

Konya Medova Hospital, Neonatology, Konya-Turkey ${ }^{4}$

Radiological imaging in the neonatal period provides very useful clues for differential diagnosis and diagnosis. Here, the findings of antenatal bilateral symmetric hemorrhage and right subdural hemorrhage in the bilateral temporal lobe on MRI were evaluated as consistent with alloimmune-autoimmune neonatal thrombocytopenia. The case was presented because of its rarity.

\section{Case}

A 2800-year-old mother was taken to the intensive care unit at 4100 grams after spontaneous vaginal delivery due to spontaneous vaginal delivery at 37 weeks of gestation with G4P2Dc1. He was treated for hypothermia with severe acidosis. He was treated with severe pulmonary hypertension. There was Rh incompatibility between mother and baby. In the BK automatic count Hb 6.2 Hct 17.1 BK 161,000 / mm3. Platelet 45,000 / mm3. He had severe hemolysis findings, left shift, and diffuse normoblasts. PT, PTT prolonged, CK and troponin I were found high. The patient was treated with hypothermia and three blood exchanges were performed. IVIG treatment was performed before the first blood exchange. In the first month of hospitalization, she was treated with Klebsiella pneumonia and sepsis treatment for three weeks. When the patient is stable, brain MRI was performed. According to MRI report, "Hemorrhage and related porencephalic cystic parenchymal changes and signal differences due to gliosis are seen in the bilateral temporal lobe. Two mm thick subdural hemorrhage is seen in the left frontotemporal region. There was no evidence of hemorrhage in other areas of the cerebral parenchyma. Although imaging typically suggests alloi-immuno-isoimmune thrombocytopenia, we could not prove it to be laboratory-specific because of the patient's complex clinic.

Early neonatal period should be taken into consideration in the differential diagnosis because many causes have similar findings. Radiological imaging provides important clues in these cases. 


\section{E1347 - AMNIOTIC FLUID CONCENTRATIONS OF SOLUBLE ENDOGLIN AND ENDOTHELIAL CELL SPECIFIC MOLECULE 1 IN PREGNANCIES COMPLICATED WITH NEURAL TUBE DEFECTS \\ Ovayolu A. $^{1}$ \\ Cengiz Gokcek Women, Obstetrics and Gynecology, Gaziantep-Turkey ${ }^{1}$}

\section{Objective}

To determine the concentrations of soluble endoglin (sCD105) and endothelial cell-specific molecule-1 (ESM-1) in the amniotic fluid (AF) of pregnant women, and to investigate the relationship between these concentrations with neural tube defects (NTDs).

\section{Methods}

AF concentrations of sCD105 and ESM-1 were measured in the study group, which included 60 pregnant women complicated with NTDs; and 64 pregnant women with unaffected healthy fetuses (control group). The AF concentrations of sCD105 and ESM-1 in both groups were measured using enzyme-linked immunosorbent assay and compared.

\section{Results}

The demographic data and biochemical parameters in amniotic fluid of the groups were compared (Table 1). There were no significant differences in terms of the mean AF concentrations of sCD105 and ESM- 1 between the groups ( $\mathrm{p}=0.141, \mathrm{p}=0.084$, respectively). No statistically significant relations were detected between sCD105 and ESM-1 ( $p=0.907)$. There was a significant difference between the AF sCD 105 concentrations in those with gestational age $<24$ weeks $(n=101)$ and $\geq 24$ weeks $(n=23)\left(X_{-}^{-}(<24)=76.35 \pm 126.62\right.$ vs $\left.\mathrm{X}_{-}(\geq 24)=39.87 \pm 58.32, \mathrm{p}=0.041\right)$. AF ESM-1 concentrations were found to be statistically significant in the gestational age $\quad<22$ weeks $(n=90)$ and $\geq 22$ weeks $(n=34)$ groups $\left(X_{-}(<22)=135.91 \pm 19.26\right.$ vs X__ $\left.(\geq 22)=148.56 \pm 46.85, p=0.035\right)$. A positive and low-level relation at a statistically significant level was determined between the gestational age and AF ESM-1 concentration in the study group $(r=0.257 ; \mathrm{p}=0.048)$. When the AF ESM-1 concentrations of the patients only in the study group who were at gestational age $<24$ weeks $(\mathrm{n}=42)$ and $\geq 24$ weeks $(\mathrm{n}=18)$ were compared, a statistically significant difference was detected $\left(\mathrm{X}_{-}^{-}(<24)=137.46 \pm 19.80\right.$ vs. $\left.\mathrm{X}_{-}(\geq 24)=160.19 \pm 60.94, \mathrm{p}=0.033\right)$. When only the patients in the study group were divided into two groups as cranial $(n=31)$ and spinal $(n=29)$ NTDs, the sCD105 concentrations showed no statistically significant differences $\left(X_{-}\right.$cranial $=70.97 \pm 128.30$ vs. $X$ spinal $\left.=35.13 \pm 51.22, p=0.166\right)$. When only the patients in the study group were divided into two groups as cranial and spinal NTDs , the ESM -1 concentrations showed no statistically significant differences $\quad\left(X_{-}\right.$_cranial=137.59 \pm 21.11 vs. $\mathrm{X}$ spinal $=151.42 \pm 49.81, \mathrm{p}=0.162$ ).

\section{Conclusion}

AF concentrations of sCD105 and ESM-1 were not associated with the development of NTDs. Unlike studies that reported that ESM-1 concentrations decreased in maternal plasma with the increase in gestational age, we determined an increase that was proportionate to gestational age in AF. 
E1396 - CONGENITAL HYDROCEPHALUS ON ARTERIOVENOUS MALFORMATION A CASE REPORT.

Oancea M. ${ }^{1}$, Blaga L. ${ }^{2}$, Bodean D. ${ }^{3}$, Diculescu D. ${ }^{1}$, Ciortea R. ${ }^{1}$, Mihu D. ${ }^{1}$

Umf Cluj-napoca, Obstetrics and Ginecology, Cluj-napoca-Romania ${ }^{1}$

Umf Cluj-napoca, Neonatology, Cluj-napoca-Romania ${ }^{2}$

Novogyn Cluj-napoca, Obstetrics and Ginecology, Cluj-napoca-Romania ${ }^{3}$

Congenital hydrocephalus is the result of the imbalance between the production and the resorption of the cerebrospinal fluid, which causes the increase of the intracranial pressure with the expansion of the ventricular system.

We report on a case of a 27-year-old patient, diagnosed in the 35th weeks of gestation with fetal hydrocephalus. Fetal MRI reveals associated hemorrhage in the right lateral ventricle. Term birth by cesarean section of a female fetus with Apgar 9. Postnatal ultrasound confirms the diagnostic and computer tomography reveals a tumor formation in the cortex of the right lateral ventricle without being able to exclude a subacute/chronic hematoma. On the 6th day of life, surgery is performed. The ventricular hematoma was evacuated, and an arteriovenous malformation was identified as a capable bleeding source. The left lateral ventricular dilatation requires insertion of a left ventricular external drainage. The favorable evolution made it possible to discharge the patient on day 44 of life. The uncertain prognosis, with the probability of normal psychomotor development after the surgical resolution of $60-80 \%$, depends on the severity, precocity of the installation and the progressive character.

In our case, at the age of 1 year and 6 months, motor development corresponds to a 3-monthold baby and the clinical examination reveals microcephaly, hemiparesis and left hemianopsy, associated with focal epileptic symptoms. 
E1403 - SUBSTANCE ABUSE DURING PREGNANCY AND ITS EFFECT ON CARDIOVASCULAR ADAPTATION AND NEURODEVELOPMENT IN NEONATE $\underline{\text { Košec V. }}{ }^{1}$, Potkonjak A. ${ }^{1}$, Knezović Z. ${ }^{1}$, Ivičević Bakulić I. ${ }^{2}$, Djaković I. ${ }^{1}$, Sabolović Rudman S. ${ }^{1}$

University Hospital Center Sestre Milosrdnice, Gynecology and Obstetrics, Zagreb-Croatia ${ }^{1}$ University Hospital Center Sestre Milosrdnice, Anesthesiology and Intensive Care, Zagreb Croatia $^{2}$

\section{Introduction}

Comprehensive and high quality perinatal health care requires the care and follow-up of pregnant substance abusers. Proper treatment requires knowledge of true epidemiological characteristics, socioeconomic conditions and psychological factors that have led to addiction. Whether pregnancy is planned or not, the monitoring and treatment of this kind of risk pregnancies should be directed toward successful fetal outcome. Although positive short-term neonatal outcomes are known, the emergence of neonatal abstinence syndrome (NAS) is described in up to the $90 \%$ of cases in literature.

Most recently, the variation of intensity of NAS symptoms and the need for pharmacological therapy are explained by the epigenetic changes and the degree of methylation of MOR gene ( $\mu$-opioid receptor). In addition to the short-term symptoms of NAS, it is necessary to consider long term effects of neonatal exposure as well as outcomes of negative effect of opioids on tissue development.

\section{Objective}

The case-control study aimed to establish the correlation between substance abuse during pregnancy and short term perinatal outcome as well as the specific cranial ultrasonographic and echocardiographic findings in neonates.

\section{Methods}

Data for opioid - dependent pregnant women was collected from the archives of the Department of Obstetrics and Gynecology of University Hospital Centre Sestre Milosrdnice between 2016. and 2019. Among the 14 substance users, 10 of them were taking buprenorphine and 4 were using methadone. Given data was compared with the control group of 14 gravidae who did not use any of addictive substances. Patients of control group were sampled in a way that the first following patient with same gestational age was included in study. Categorical data is displayed in absolute and relative frequencies. Data analysis was performed using Mann - Whitney and Chi - squared test. Statistical analysis has been performed using the Statistical Package for Social Sciences (SPSS V20).

\section{Results}

Substance exposure was not significantly associated with mode of delivery or Apgar score. Use of addictive drugs was associated with lower birth weight $(\mathrm{p}<0.01)$. However, there were no statistically significant differences in Ponderal index. All except one patient experienced NAS. The reported symptoms were irritability, tremors, high pitched crying, gastrointestinal symptoms and sweating. The mean age at onset of symptoms was 44.6 hours. The use of methadone was associated more cranial ultrasound abnormalities then buprenoprhine but with less reported cardiac defects. Most commonly reported cardiac anomalies were patent ductus arteriosus (PDA) and patent foramen ovale (PFO). Analyzing cranial sonograms, increased periventricular echogenicity and intracranial hemorrhage grade 1 or 2 were prevalent among neonates prenatally exposed to opioids. 


\section{Conclusion}

Although this study was conducted on a small number of subjects, it highlights the need for a more precise and accurate monitoring of drug-addicted pregnant women. 
E1454 - THE NOMOGRAM OF THE CAVUM SEPTUM PELLUCIDUM AT 1528 WEEKS OF GESTATION

Arisoy.$^{1}$, Yayla M. ${ }^{2}$

Memorial Atasehir Hospital, Perinatology, Istanbul-Turkey ${ }^{1}$

Acibadem International Hospital, Perinatology, Istanbul-Turkey ${ }^{2}$

\section{Objective}

To obtain nomogram of the cavum septum pellucidum (CSP) width at 15-28 weeks of gestation with known prognosis of normal pregnancies

\section{Methods}

The CSP width and other routine biometric measurements of 6042 structurally normal fetuses at 15-28 weeks of gestation were measured by transabdominal ultrasonography. The distribution of the CSP width is established according to gestational weeks and percentiles between 15-28 weeks are calculated. Statistical analysis was performed using Statistical Package for the Social Sciences Version 22 (SPSS Inc., Chicago, IL, USA).

\section{Results}

The mean gestational age was $21 \pm 1.7$ weeks and mean biparietal diameter (BPD) was $50.2 \pm$ $5.8 \mathrm{~mm}$. The CSP width ranged from 1.6 to $7.7 \mathrm{~mm}$ between 15 and 28 weeks of gestation and the mean CSP width was $4.1 \pm 0.8 \mathrm{~mm}$. The CSP width is linearly increased between 1528 weeks. Significant correlation was also found between the CSP width and gestational weeks $(\mathrm{GW})(\mathrm{CSP}=\mathrm{GW} X 0,2705-1,6121 ; \mathrm{R}=0.62 ; \mathrm{p}<0.01)$ and $\mathrm{BPD}(\mathrm{CSP}=\mathrm{BPD} \mathrm{X}$ $0,0859-0,273 ; R=0.651 ; p<0.01$ ). A significant difference in CSP width were observed between gestational age groups and the percentiles for CSP width were calculated for 15-28 weeks of gestation. The ninety-fifth percentiles for CSP width were $3.7-7 \mathrm{~mm}$ at 15-28 weeks of gestation.

\section{Conclusion}

The CSP width showed a linear increase between 15-28 weeks of gestation. Gestational weeks should be taken into consideration during the evaluation of the CSP width. 


\title{
Obstetrics - Aneuploidy and fetal anomalies - first trimester
}

\author{
E1209 - PRUNE BELLY SYNDROME DIAGNOSIS IN MONOCORIONIC TWINS IN \\ FIRST TRIMESTER \\ Urtasun M. ${ }^{1}$, Abián N. ${ }^{2}$, Gastón B. ${ }^{1}$, Ruiz M. ${ }^{1}$, Bazán M. ${ }^{1}$, Barrenetxea J. ${ }^{1}$, Zabaleta I. ${ }^{1}$, \\ Pedroarena I. ${ }^{1}$, Pérez B. ${ }^{1}$, García S. \\ Complejo Hospitalario De Navarra, Servicio Obstetricia Y Ginecología, Pamplona-Spain ${ }^{1}$ \\ Hospital Reina Sofía, Servicio Obstetricia Y Ginecología, Tudela-Spain ${ }^{2}$
}

\section{Introduction}

The Prune-Belly syndrome is a congenital disorder defined by abdominal muscle deficiency, severe urinary tract abnormalities and bilateral cryptorchidism in males. The incidence is approximately 3.8 cases per 100,000 live births. The genetic basis is unknown, but a recessive $\mathrm{X}$-linked defect is suggested because of it typically occurs in males, although there are a few cases reported in females. The pathogenesis remains unknown, but it is thought to be due to a mesenchymal genetic defect.

The major renal change is dysplasia that affects a variable portion of renal tissue, and it may not be evident before 16 weeks gestation. The bladder is usually enlarged and nontrabeculated, and has thickened walls with smooth muscle hypoplasia. In the first half of gestation, oligohydramnios due to lack of sufficient urine production promotes mechanical compression of the fetus and inhibits thoracic expansion and the e lungs remain hypoplastic. Partial aplasia or hypoplasia of the abdominal muscles are characteristic of Prune Belly Syndrome.

\section{Case}

This was a 33 year old Hispanic woman with no personal history of interest (except for obesity) in her second pregnancy. She had a normal previous pregnancy with at term delivery by cesarean section due to placental abruption.

She came to gestational control in her $13+2$ week. This pregnancy was spontaneous and with a normal course so far. The first-trimester blood analysis didn't show remarkable alterations, without having undergone biochemical screening of chromosomopathies due to patient's rejection. A monoamniotic monochorionic gestation was found in the ultrasound, with two live fetuses with crown-rump length (CRL) of $59 \mathrm{~mm}$ and $49.5 \mathrm{~mm}$ (smaller than amenorrhea), and both of them had an anomaly compatible with megabladder. Chorial biopsy was performed without complications, and the karyotype result was normal XX. The findings and possibilities were explained to the patient and she decided to interrupt the pregnancy. The anatomopathological study demonstrated a monoamniotic monochorionic gestation, with both female fetuses with complete defect of the abdominal wall and megabladder.

\section{Conclusion}

The renal dysplasia, the urinary tract abnormalities and the presence of pulmonary hypoplasia are the principal clinical manifestations and those that determine the prognosis. They result in recurrent episodes of urinary tract infections, urosepsis, and varying degrees of renal and respiratory insufficiency.

The diagnosis is typically made by antenatal ultrasonographic examination between 20 and 30 weeks. Although nowadays the vaginal sonography allows visualization of the kidneys and the urinary drainage system as early as 11 to 13 weeks gestation. The management depends on the severity of the clinical findings. Patients with Prune-Belly syndrome have had generally a poor prognosis. The overall mortality was between 30 and $50 \%$ with the majority of deaths confined to the perinatal period. 


\section{E1210 - GASTROSCHISIS CASE REPORT}

Pérez Munárriz B. ${ }^{1}$, Garcia Francés S. ${ }^{2}$, Urtasun Murillo M. ${ }^{1}$, Abian Franco $N .{ }^{1}$, Gastón B. ${ }^{1}$, Ruiz M. ${ }^{1}$, Bazán M. ${ }^{1}$, Barrenetxea J. ${ }^{3}$, Zabaleta I. ${ }^{3}$, Pedroarena I. ${ }^{3}$

Complejo Hospitalario Navarra, Gynecology and Obstetrics, Pamplona-Spain ${ }^{1}$

Complejo Hospitalario Navarra, Gynecology and Obstetric, Pamplona-Spain ${ }^{2}$

Hospitalario Navarra, Gynecology and Obstetrics, Pamplona-Spain ${ }^{3}$

\section{Introduction}

Gastroschisis is a full-thickness paraumbilical abdominal wall defect usually associated with evisceration of bowel and sometimes other abdominal organs. Bowel herniation may lead to a variety of intestinal abnormalities. Other common potential sequelae of gastroschisis include growth restriction (30 to $60 \%$ ), spontaneous preterm birth (30 to 50\%), and fetal demise (3 to $6 \%)$. After birth, the newborn requires special care because of the exposed bowel. The prognosis is generally very good. We present prenatal diagnosis and management of fetal gastroschisis and its good evolution.

\section{Case}

We present a 19-year-old pregnant woman, G1P0A0, diagnosed with fetal gastroschisis in the first trimester of pregnancy (12 weeks). An elevated maternal serum alpha fetoprotein level (MSAFP) is associated with this finding. Obstetricians and Pediatric Surgeons inform parents of fetal malformation, complications and fetal and postnatal prognosis. Control of pregnancy is performed in the Obstetric High Risk Consultation.

Serial ultrasounds are performed every 2-4 weeks to follow fetal growth and amniotic fluid and also obtain targeted views of the intra- and extra-abdominal bowel to look for significant dilatation, thickening, or edema. In third trimester (33 week), a slight increase is found in diameter of the distal intestinal loop at the ultrasound study and fetal lung maturation is performed. Worsening of prognosis ultrasound markers occurs at 35th week of gestation so it is decided to perform a labor induction with dinoprostone and oxytocin. Finally, a vaginal delivery is performed with a female newborn of 2020 gr, APGAR 9/10. A few hours of birth a reduction and primary closure of laparoschisis and ileo-colostomy are performed by Pediatric Surgeons. The newborn remains in the Neonatology Unit for 2 months with good recovery. At 4 months of age the girl is operating for stoma closure.

\section{Conclusion}

Gastroschisis and omphalocele are the most common fetal abdominal wall defects: the prevalence of each is approximately 3 to 4 per 10,000 live births/fetal deaths/stillbirths/pregnancy terminations. The pathogenesis of gastroschisis is unknown. There is an inverse association between maternal age and incidence of fetal gastroschisis, with the highest prevalence in whites and births to women under age 20 years. Prenatal diagnosis is based on sonographic visualization of a paraumbilical abdominal wall defect with visceral herniation. The combination of ultrasound examination and MSAFP screening detects at least 90 percent of cases. The decision on timing of delivery is based on a combination of factors, including gestational age, ultrasound findings, and fetal testing results. The mean gestational age at spontaneous labor in pregnancies complicated by gastroschisis is in the 36th week of gestation. Coordinating delivery at a tertiary care center provides optimal conditions for the neonate. 
E1282 - PRENATAL DIAGNOSIS OF INIENCEPHALY

Özalp M. ${ }^{1}$, Özbay G. ${ }^{2}$, Osmanağaoğlu M. ${ }^{1}$

KTU, Perinatology, Trabzon-Turkey ${ }^{1}$

KTU, Obstetrics and Gynecology, Trabzon-Turkey ${ }^{2}$

\section{Objective}

Iniencephaly is a disorder of the cervical spine consisting of congenital cervical synostoses, fixed retroflexion of the head, severe cervical lordosis, and varying degrees of deficits of the dorsal occiput and cervical vertebrae. In iniencephaly, developmental disturbances of the neural tube and spine arise very early in embryogenesis, generally between day 26 and day 30 after conception. Ultrasonography and serum or amniotic $\alpha$-fetoprotein levels can be used to detect this condition in utero.

The aim of this case is to contribute prenatal diagnosis and management of fetal iniencephaly.

\section{Case}

A 38-years-old G3P2 pregnant was referred to our perinatology clinic at $11+6$ weeks of gestation for first trimester scan. Her first baby had hydrocephalus and craniosynostosis in her medical story. She didn't use folic acid in her pregnancy. Sonographic examination showed a fixed flexion of the head with an upturned face (stargazer appearance) and lemon sign. Cervical and thoracic vertebrae showed incomplete formation or closure; the occipital bones seem fused with the cervicothoracic vertebrae. We performed chorion villus sampling (CVS). After 5 weeks the pregnant came up with CVS result. CVS revealed normal karyotype (46, $\mathrm{XX}$ ). The pregnant's never been in control over this period. At 17 weeks of gestation ultrasound examination showed fetus was ex. Termination was made to the pregnant woman.

\section{Conclusion}

Iniencephaly is a rare NTD. Advanced maternal age, folic acid deficiency and a history of anomalous birth are the factors that increase the risk. Associated abnormalities of the CNS include anencephaly, encephalocele, hydrocephalus, hydromyelia, microcephaly agenesis of the cerebellar vermis, and presence of cerebellar cyst. The abnormal fetal growth occurring at such an early stage in gestation might be the reason for the multiple associated malformations, owing to subsequent hindering and cramping of structures related to the retroflexed fetal neck. Iniencephaly should be distinguished from extreme hyperextension of the fetal head, which may resolve spontaneously, and Klippel-Feil syndrome. Because of the hyperextension of the head, dystocia may occur.

Iniencephaly is a rare neural tube defect that is often lethal, ending in either a stillbirth or a neonatal death. Several infants with iniencephaly have had close relatives with either anencephaly or myelomeningocele. While usually a lethal malformation, affected children and even adults with normal intelligence have been reported. 


\section{E1283 - OMPHALOCELE IN EDWARDS SYNDROME A CASE REPORT}

Estefanía Díaz A. ${ }^{1}$, Otaño Ruiz J. ${ }^{1}$, Pedroarena Lecumberri I. ${ }^{1}$, Ruiz García M. ${ }^{1}$, Abián Franco N. ${ }^{2}$

Complejo Hospitalario De Navarra, Gynaecology, Pamplona-Spain ${ }^{1}$ Hospital Reina Sofía, Gynaecology, Pamplona-Spain ${ }^{2}$

\section{Introduction}

Edwards Syndrome is a genetic disorder caused by the presence of an extra 18th chromosome. The incidence varies from 1 in 6,000 to 1 in 8,000 births, and it is the 2nd most common autosomal trisomy after Down's syndrome. It is a polymalformative syndrome characterized by a short survival, most of the foetuses die before birth and less than $5 \%$ of the newborns survive the first year.

\section{Case Description}

A 39-year-old woman with history of a previous caesarean delivery and without any other medical history of interest. During the first trimester ultrasound in the 13th week of pregnancy, a big omphalocele with intestines, stomach and liver protruding through it is described (Figure 1). The heart is tractioned by the omphalocele, avoiding a correct display of the four cardiac cavities. A cystic hygroma of $5.17 \mathrm{~mm}$ is also detected (Figure 2). The risk of chromosomopathies calculated with the first-trimester combined test is increased (1/11), so an amniocentesis is indicated for a prenatal diagnosis. After explaining the association between the findings in the ultrasound and the high risk of chromosomopathies to the patient, she refuses to make an amniocentesis as she will not interrupt the pregnancy despite the results for religious motives. In the successive visits the fetal heartbeat is checked. In the second trimester ultrasound we detect intrauterine growth restriction, the omphalocele (Figure 3), absence of the cavum septum pellucidum, and a single ventricle with transposition of the great arteries. In addition, the foetus presents rocker bottom feet and clenched hands. In the 30th week of pregnancy the diagnose of stillbirth is made, so our patient accepts the induction of labour. The birth goes well, confirming the findings in the ultrasound after the fetal autopsy. A molecular genetic study is made, confirming the diagnosis of trisomy 18, also known as Edwards syndrome.

\section{Conclusion}

Most cases of Edwards syndrome are suspected during the 1st trimester ultrasound due to tests which calculate the risk of chromosomopathy using the maternal age, markers in maternal serum and abnormal ultrasound findings. The diagnosis is made analysing the fetal chromosomes using material obtained by amniocentesis, corial biopsy or cordocentesis. In this case, the diagnosis is made after the birth as the patient refuses diagnostic techniques during pregnancy. 
E1407 - ADVANCES IN PRENATAL DIAGNOSIS AND DECISION TO TERMINATE PREGNANCY AT UNIVERSITY CLINIC OF GYNECOLOGY AND OBSTETRICS Daneva Markova A. ${ }^{1}$, Bajaldzieva S. ${ }^{1}$, Nikolovska K. ${ }^{1}$, Koteva Mirakovska M. ${ }^{1}$, Nikolovska E. ${ }^{1}$, Sivakov B. ${ }^{1}$, Atanasovski Z. ${ }^{1}$

University Clinic of Gynecology and Obstetrics, High Risk Pregnancy, Skopje-Macedonia ${ }^{1}$

\section{Objective}

The purpose of prenatal diagnosis is to obtain the appropriate information regarding prognosis, risk of recurrence, and potential therapy, and thus achieve optimum outcome for the fetus and the mother. Making a decision to terminate pregnancy is always on the parents' indication that the fetus with an increased nuchal translucency may have a normal karyotype at the start of diagnosis, a range of malformations that may develop later in pregnancy and after screening for organic malformations of 20-24 gestational week, as well as a long list of explanations for possible consequences in relation to the fetus if pregnancy is not terminated.

\section{Methods}

In the article, interruptions of pregnancies were considered during one year, 2017, at the Clinic for Gynecology and Obstetrics, the same systematized after gestation weeks. Before any termination of pregnancy amniocentesis was performed in the clinic and all other diagnostic examinations according to the week that are within the scope of the Clinic. The purpose of the review is to begin the termination of pregnancy, determined using diagnostic facilities before the interruption. Fetal anomalies divided into four major groups: cardiac defects, nervous system defects, chromosomopathies, others (covering abdominal wall defects, urinary system, hydrops, dysplasia, IVGR, multiple anomalies).

\section{Results}

According to the data on termination of pregnancy at the University Clinic of Gynecology and Obstetrics, in 2017, the total number of interruptions of pregnancy is greatest in the period from 20.1 to 24 gestational week, or $44 \%$ of all interruptions of pregnancy. Aborted pregnancies above 28 weeks of gestation are $11 \%$, most of them due to defects in the nervous system (34\%), and equally represented cardiac defects and chromosomopathies. Neural tube defects (anencephaly, spina bifida, cephalocele, holoprosencephalus, acrony ...) are the second most common fetal abnormalities after cardiac anomalies (Williams, Cragan, 2009, Dolk, 2010), or interruption of pregnancy due to the same in our examined group $40 \%$ of all interruptions over one year. Discontinuation of pregnancy due to a cardiac defect in the examined group is $10 \%$ of all interruptions in a year, due to cardiopathia complex.

\section{Conclusion}

The determination of Nuchal translucency and its elevated values are only the beginning which points to additional diagnostics and confirmation of fetal abnormalities. Further biochemical screening in the second trimester, ultrasound echocardiography and screening for structural defects of other organs and organ systems complement the diagnosis of fetal abnormalities in subsequent weeks of pregnancy, unless an abortion occurred in early pregnancy or fetus in utero death. Amniocentesis affects parents' decision-making, their desire for prolonged pregnancy, or a decision to prolong pregnancy with otherwise unhealthy organic anomalies and heart failure, which at some point in the pregnancy would end up in fetal death or soon after birth. Reduction of the percentage of interruption of pregnancy due to anomalies that are the first in the presence of the fetus is due to the progress in the operative resolution of the fetus. 


\section{E1418 - NOONAN SYNDROME ANTENATAL DIAGNOSIS AND CLINICAL IMPLICATIONS}

Pontikaki A. ${ }^{1}$, Neonakis S. ${ }^{1}$, Velegrakis A. ${ }^{2}$, Papadopoulou E. $^{3}$, Sifakis S. ${ }^{4}$

University of Crete, Department of Obstetrics and Gynecology, Heraklion-Greece ${ }^{1}$

Venizeleio General Hospital, Department of Obstetrics and Gynecology, Heraklion-Greece ${ }^{2}$

University of Crete, Department of Pediatrics, Heraklion-Greece ${ }^{3}$ Mitera Maternity

Hospital, Heraklion-Greece ${ }^{4}$

\section{Objective}

Noonan syndrome is a rare genetic disorder with its prevalence estimated between 1 in 1,000 to 2,500 live births, affecting equally male and female infants. It is an autosomal dominant disorder mainly, yet both autosomal recessive inheritance and de novo cases have been reported. The syndrome presents a clinical and genetic heterogeneity, characterized by short stature, mild facial malformations, congenital heart abnormalities, and a variety of cognitive deficits. Mutations in seven genes have so far been associated with Noonan syndrome, especially those related to the PTPN11 gene. These mutations are thought to cause dysregulation in the RAS-MAPK signaling pathway, which also appears to be involved in the pathogenesis of other syndromes such as Leopard syndrome (LS), Noonan-like(with loose anagen hair, NS/LAH) syndrome, Costello's syndrome (CS), Cardio-Facio-Cutaneous syndrome (CFCS), Neurofibromatosis Type I (NF1) and Legius syndrome (LS). We present 3 cases of Noonan's syndrome, that were diagnosed antenatally as a result of detection of suspicious ultrasonographic findings in the fisrt trimester of pregnancy, and discuss the role of detailed screening by a specialist in fetal medicine, as well as the multiple challenges of a comprehensive parental counseling, once the diagnosis has been confirmed by molecular karyotype.

\section{Methods}

In the first trimester scan of 12-13 weeks, performed in a fetal-maternal referral unit, sonographic findings such as cystic hygroma in one case and increased nuchal translucency in two cases, lead to Chorionic Villous Sampling (CVS).

\section{Results}

In all 3 cases, molecular karyotype revealed the characteristic PTPN11 gene mutation suggestive of Noonan syndrome. An extensive parental counseling by a specialist in fetalmaternal medicine, as well as by a pediatrician with expertise in congenital malformations and genetic disorders followed, regarding the prognosis, the clinical heterogeneity and the broad spectrum of manifestation of signs and symptoms, the treatment options available after birth, the implications on future pregnancies and the need for genetic testing of both parents. All couples decided to terminate pregnancy, referring as their major concern the possibility of a severe cognitive disorder.

\section{Conclusion}

Diagnosis of Noonan Syndrome is mainly based on genetic testing, however, antenatally, due to absence of specific ultrasound findings, it should be suspected in any case of first trimester increased nuchal translucency or cystic hygroma, or increased or persistent nuchal fold during the second trimester of pregnancy. The role of ultrasonographic screening is indispensable, especially in the absence of a family history, to guide further testing to confirm diagnosis, counseling, clinical management and future implications. 


\title{
Obstetrics - Noninvasive prenatal test ( Fetal DNA )
}

\author{
E1186 - TWO YEARS OUTCOMES OF NON INVASIVE PRENATAL TEST OF \\ TRISOMY \\ Sarreau M. ${ }^{1}$, Diffaza F. ${ }^{2}$, Goua V. ${ }^{2}$ \\ Hospital Center, Obstetrics, Angouleme-France ${ }^{1}$ \\ University Hospital Center, Obstetrics, Poitiers-France ${ }^{2}$
}

\section{Objective}

Non invasive prenatal testing for trisomy 21 (NIPT) by cell free DNA analysis exist in European countries. First of all in France, it was available only in research studies, then paid and very expensive. It has been officially published by French High Health Authorities in 2017, and is now supported by French health assurance since January 2019. We studied our practices and results, and pregnancy outcomes before 2019.

\section{Methods}

Our public structure accepted to finance non invasive prenatal testing in maternal blood for trisomy 21 from 2017. This is transversal cohort.

NIPT is allowed only without fetal abnormalities by ultrasonography examination after first or second trimester screening trisomy blood test in France. It is based on combinated test of factors including nuchal translucency if possible, hormonal analysis (PAPPA, HCG or AFP) and maternal age. NIPT used to be proposed when early screening test is between 1/50 and $1 / 1000$ with different level of risks. Invasive intervention was still indicated for karyotype by amniocentesis for test $>1 / 50$, but NIPT could be done anyway. We did 408 tests, and could get 348 NIPT results.

\section{Results}

On 348 NIPT: 5 were done for wrong indication, 5 for antecedent, 56 for patients with late pregnancy declaration without first trimester ultrasound, 53 for multiple pregnancy (39 bichorionic-biamniotic, 12 monochorionic-biamniotic, 1 monochorionic-monoamniotic, 1 triple), 196 for intermediate risk between 1/50-1/1000, 13 for high risk >1/50, 20 lost of view. In only 3 cases NIPT was undetermined and needed to be done twice and was negative and it couldn't be returned in one case in intermediate risk (the patient chose not to perform the amniocentesis and to continue the ultrasound surveillance).

For all patients mean age was 29 years old; parity 1,8; body mass index was 28 ; median term of delivery was 39SA+4 gestation week and birth weigh 2950 grams.

Surprisingly, only 1 trisomy 21 was found in high risk group ( $>1 / 50)$, other ultrasound follow up and neonatal outcomes were normal.

False positive test were 1 for trisomy 21 and trisomy 18, none false negative: sensibility was $100 \%$ and specificity $99,7 \%$, predictive positive value respectively 0,8 and 0,66 . It was more often in intermediate group (without identified predictive factor).

Multiple pregnancies did not create technical problem.

According to recommendations, NIPT could avoid 185/196 (94,3\%) useless amniocentesis which used to be done when screening was $>1 / 250$.

\section{Conclusion}

In France, this strategy of early combined test and indications of NIPT allows to select patient at different type of risk. It is a at time interesting for financial reasons and to avoid useless $94 \%$ amniocentesis. NIPT is safe, with good sensitivity and specificity. 
Unfortunately it could be as well a source of stress for patient and difficulties to invest pregnancy for false positive NIPT. Further information could be given for patients before prescription of NIPT. 


\section{E1253 - CASE REPORT VALUE OF CFDNA TESTING IN DETECTING OTHER CHROMOSOMAL ABRNORMALITIES}

Jelcic D. ${ }^{1}$, Podobnik Brlečić P. ${ }^{1}$, Podobnik M. ${ }^{1}$, Kurdija K. ${ }^{1}$, Gebauer Vuković B. ${ }^{1}$ Specijalna Bolnica Podobnik, Ob\&gyn, Zagreb-Croatia ${ }^{1}$

Using cfDNA testing in detection of Trisomy 21, 18 and 13 has shown high PPV (high detection rate with low false positive rate). In our private Hospital many women opt for NIPT with the possibility to report incidental findings on other chromosomes as well, and we report 2 cases where using MPSS method has given us clinical benefit.

\section{Case 1}

35-year old women, second pregnancy, opted for NIPT at 12 week of pregnancy, with normal first trimester ultrasound screening (CRL $58 \mathrm{~mm}$, NT $1.2 \mathrm{~mm}$ ). First pregnancy was 10 years ago and she delivered vaginally healthy female child. NIPT result (NIFTY plus test, MPSS method) was high risk in possibility of fetal trisomy 5 with fetal fraction $8.94 \%$. In reviewing publications it has been reported that mosaic trisomy 5 is rare chromosomal anomaly syndrome with a variable phenotype ranging from clinically normal to patient presenting intrauterine growth retardation, congenital heart anomalies, dysmorphic features and other congenital anomalies (eventration of diaphragm, agenesis of corpus callosum, cloverleaf skull, clinodactyly, anteriorly placed anus).

At 15 week of gestation she underwent amniocentesis and control ultrasound that showed SUA (single umbilical artery) and slight growth retardation, but with normal visible fetal morphology. Microarray report confirmed mosaic trisomy chromosome 5, estimated size $20 \%$, and FISH method in cultured cells confirmed 6\% mosaic chromosome 5. At 18 week of gestation she was admitted in the hospital with missed abortion for termination of pregnancy.

\section{Case 2}

25-year old healthy women, first pregnancy, wanted NIPT at 12 weeks of gestation, with normal first trimester ultrasound screening (CRL $65 \mathrm{~mm}$, NT $1.1 \mathrm{~mm}$ ). NIPT result (NIFTY plus test, MPSS method) was high risk for duplication of chromosome 9 (9p24.3-P13.1, 38.68 M), with fetal fraction $13.19 \%$.

Duplication 9p may affect facial dysmorphismus and abnormalities of the fingers, toes, nails and heart failure.

At 16 week of gestation ultrasound screening showed ventriculomegaly, cleft lip and palate, no visible stomach and single umbilical artery. At that gestational age amniocentesis was done and duplication (or triplication, also a presence of possible mosaicism cannot be excluded) of chromosome 9p24.3-p13.1 was detected in prenatal array CHG analysis. The result was concordant with NIFTY testing results. Additionally, chromosome analysis of amniotic fluid cells using standard G-banding technique showed karyotype 47,XY,+psu $\operatorname{dic}(9 ; 9)(q 12 ; q 12)$ (unbalanced structural aberration). At this moment the patient is 21 weeks of ongoing pregnancy, with no other data.

\section{Conclusion}

We can conclude that although cfDNA testing has been validated for screening of common trisomies, in case that we perform the extended panel that some laboratories offer, we could find useful clinical information that has been confirmed by invasive testing. 
E1254 - NON INVASIVE PRENATAL TESTING (NIPT) FOR COMMON CHROMOSOMAL ANEUPLOIDIES DATA FROM A SINGLE CENTER IN A ROUTINE SCREENING POPULATION

Jelcic D. ${ }^{1}$, Podobnik Brlečić P. ${ }^{1}$, Podobnik M. ${ }^{1}$, Kurdija K. ${ }^{1}$, Gebauer Vuković B. ${ }^{1}$

Specijalna Bolnica Podobnik, Ob\&gyn, Zagreb-Croatia ${ }^{1}$

\section{Objective}

Non- invasive prenatal testing (NIPT) by analysis of cell-free DNA (cfDNA) from maternal blood has shown promise for highly accurate detection of common fetal trisomies. We assessed the performance of NIPT for common chromosomal aneuploidies screening in a routine pregnant population from a single center in Zagreb.

\section{Methods}

We present the results of prenatal cfDNA testing in a period from 25.03.2013 until 26.03.2019 in a private Hospital in Zagreb. Of total 1161 pregnant women on which cfDNA testing was performed, 6 were twin pregnancies. All samples were analyzed using massively parallel sequencing, in clinical laboratory of BGI -Shenzhen, China.

\section{Results}

Results were available in 1157 cases (99,66\%), among them delayed results in 31 (2,67\%), 12 needed resampling (redraw rate 1,03 \%) and only 4 'no call' result. Among 22 (1,9\%) high risk results there were 13 Trisomies 21, 2 Trisomies 18, 1 Trisomy 13, 1 45X0, 1 Cri Du Chat, 1 Trisomy 5, 1 Trisomy 2 and 1 duplication 9 chr. All cases of T21, 1 case of T18 and T13 were confirmed by karyotyping (PPV for T21 is 100\%). 2 women with high cf-DNA test result for T18 had miscarriage before karyotyping. Trisomy 2 was not confirmed by standard karyotyping but interestingly suspected trisomy 5 confirmed mosaic of trisomy 5 in $6 \%$ of cultured cells (it was a missed ab at 17 weeks of pregnancy). A suspected $5 p$ deletion and trisomy 2 was not confirmed by microarray method. There was one case of false negative $\mathrm{T} 21$, resulting in sensitivity for T21 of $92,31 \%$.

\section{Conclusion}

The performance of screening for Trisomy 21, 18, 13 and sex chromosome aneuploidies by cf-DNA testing using massively parallel sequencing is most effective screening method with high detection rates and extremely high PPV (100 \%) for Trisomy 21. Our results show extremely low redraw and 'no call' rate. 
E1417 - SHOULD FETAL ARRAY CHG ANALYSIS BE OFFERED TO PARENTS UNDERGOING INVASIVE TEST DUE TO INCREASED RISK OF SEX CHROMOSOMAL ABNORMALITY ON FETAL DNA TESTING

Api O. ${ }^{1}$, Ozgon G. ${ }^{2}$, Boza A. ${ }^{3}$, Ceyhan M. ${ }^{3}$, Cil A. ${ }^{3}$

American Hospital, Perinatology, Istanbul-Turkey ${ }^{1}$

Nesiller Genetic, Genetics, Istanbul Turkey ${ }^{2}$

American Hospital, Ob\&Gyn, Istanbul-Turkey ${ }^{3}$

\section{Objective}

To emphasize the importance of array-CGH testing in fetuses with sex chromosomal abnormalities

\section{Case}

A 32-year-old woman, G1P0, was examined for first trimester screening in the antenatal follow-up outpatient clinics. The early fetal anatomy scan revealed no abnormalities with an NT: $1,2 \mathrm{~mm}$ at 12 weeks' gestation. The parents opted for fetal DNA testing without first trimester biochemical testing. The fetal DNA test revealed increased risk for 47, XXY with a fetal fraction of $8.9 \%$. The patient underwent an amniocentesis without any complications at 15 weeks \& 3 days. The Quantitative Fluorescent PCR (QF-PCR) QF-PCR, using a set of STR markers for chromosomes 13, 18, 21, X, and $\mathrm{Y}$ revealed normal number of chromosomes. However, the conventional karyotype analysis yielded $90 \%$ mosaicism for 47 , XXY (47, --- (91)/46,--- (9)). The genetic laboratory continued array-CGH analysis upon parents' request. Meanwhile, the detailed fetal anatomy scan revealed a Blake's Pouch cyst and bilateral contracture of hands with polydactyly. Array-CHG analysis revealed uniparental isodisomy $\mathrm{X}$. The patient was given genetic counseling and the fetal abnormality was thought to be due to a X-linked single gene disorder. The parents opted for termination of pregnancy. A skin biopsy of the fetus was sent to genetic laboratory for whole-exome sequencing. The results revealed pathogenic mutation on TCTN2 gene.

\section{Conclusion}

Increased risk for sex chromosomal abnormalities detected on fetal DNA testing usually represent a dilemma both for clinicians and parents-to-be. Despite, Klinefelter syndrome usually does not necessitate termination of pregnancy, it may be reasonable to offer fetal array-CHG analysis among with conventional karyotyping if the patients consider to undergo invasive test due to increased risk of any sex chromosomal abnormality on fetal DNA testing. 


\section{Obstetrics - Aneuploidy and fetal anomalies - second trimester}

E1123 - CLINICAL VALUE OF BACTERIAL ARTIFICIAL CHROMOSOMES (BACS)-ON-BEADS (BOBS) FOR PRENATAL DIAGNOSIS OF ANEUPLOIDIES AND MICRODELETIONS

Wang Y. ${ }^{1}$, Huang X. ${ }^{1}$, Hu J. ${ }^{1}$, Qi B. ${ }^{1}$

Fuzhou Municipal First Hospital Affiliated to Fujian Medical University, Obstetrics and

Gynecology, Fuzhou-China ${ }^{1}$

\section{Objective}

To assess the clinical value of bacterial artificial chromosomes (BACs)-on-beads (BoBs) for prenatal diagnosis of aneuploidies and microdeletions.

\section{Methods}

The amniotic fluid and cultured cell samples were collected from 193 pregnant women undergoing BoBs and karyotyping simultaneously due to advanced age, abnormal ultrasound and high-risk pregnancy of serological screening. Chromosomal karyotype analysis was employed as the gold standard to evaluate the efficiency of BoBs for prenatal diagnosis of aneuploidies and microdeletions.

\section{Results}

Of the 193 pregnant women-derived amniotic fluid and cultured cell samples, the success rate of karyotyping and BoBs was both 100\%. Chromosomal karyotype analysis detected 25 fetal chromosomal abnormalities, with a $13.0 \%$ proportion, and BoBs revealed 27 fetal chromosomal abnormalities, with a $14.0 \%$ proportion. The results of BoBs were totally consistent with chromosomal karyotype analysis, and BoBs did not detect false positives or false negatives. In addition, BoBs revealed 22q11.2 microdeletion syndromes (DiGeorge Syndrome) in two samples from the pregnant women with high-risk pregnancy.

\section{Conclusion}

BoBs is a rapid, sensitive and reliable prenatal diagnosis tool for the detection of aneuploidies and microdeletions. As an alternative of karyotyping, it may be used for the detection of common aneuploidies, and the diagnosis of microdeletions as an effective complementary of karyotyping. 
E1143 - PLACENTAL CHORIOANGIOMA AS CAUSE OF SEVERE FETAL ANEMIA AND INTRAUTERINE BLOOD TRANSFUSION A CASE REPORT

Noya Galluzzo R. 1, Franco M. 1, Souza Da Correggio K. 1, Simas Abi-Saab J. 1, Serafin

Couto Vieira D. ${ }^{1}$, Coutinho Cavalieri J. ${ }^{1}$, Mariko Nakasono Molin M. ${ }^{1}$,

Koettker Silveira S. ${ }^{1}$, May Feuerschuette O. ${ }^{1}$, Trapani Jr. A. ${ }^{1}$

$\mathrm{Hu} /$ ufsc/ebserh/ Women, Gynecology and Obstetrics, Florianópolis-Brazil ${ }^{1}$

Chorioangiomas are the most common non-trophoblastic tumors of the placenta and consist of anomalous vascular proliferation of the placental tissue. The estimated incidence is $1 \%$, however clinically evident chorioangiomas are less common, being reported between 1:3,000 and 1:9,000 births. The etiopathogenesis is unknown and routine screening is not recommended. However, the presence of chorioangiomas should be considered among differential diagnosis of fetal growth restriction or delayed fetal growth in the absence of placental insufficiency. Fetal complications include non-immune hydrops, polyhydramnios, fetal growth restriction, cardiomegaly, congestive heart failure, severe anemia, thrombocytopenia, consumption coagulopathy, preterm labor, and fetal demise. Chorioangiomas are mainly perfused by fetal circulation and tumor size determines the amount of blood divergent from fetal circulation and represents the main determinant of the presence and severity of complications and perinatal outcome.

\section{Case}

A 30 year-old pregnant woman, G1, was referred to our hospital due to non-immune fetal hydrops and abnormal MCA-PSV suggestive of severe fetal anemia of unknown etiology at gestational age of $26+1$ weeks. A new ultrasound (US) scan also showed placentomegaly and a hypoechogenic placental node of approximately $6 \times 2 \times 2 \mathrm{~cm}$ with exuberant vascularization that could correspond to placental chorioangioma. She was managed with serial intravascular transfusion. The first transfusion was realized on November 30th, 2018 when it was transfused $23 \mathrm{~mL}$ of blood $\mathrm{O}$ negative, which increased fetal hemoglobin from $4.1 \mathrm{~g} / \mathrm{dL}$ to 9.4g/dL. On December 3rd, 2018, US scan demonstrated worsening of fetal anemia. Fetal hemoglobin was $4.1 \mathrm{~g} / \mathrm{dL}$. She received another intrauterine transfusion of $30 \mathrm{~mL}$ of blood $\mathrm{O}$ negative. Fetal hemoglobin after the procedure was $8.0 \mathrm{~g} / \mathrm{dL}$. After 4 days, doppler gets worse displaying severe fetal anemia. The patient was admitted to hospital for corticosteroid therapy for pulmonary maturation and to plan the interruption of gestation. Cesarean section was performed on December 10th, 2018. She gave birth to a healthy female baby, weighing 995g, Apgar score of 6 and 8, and Ballard of 27 weeks. The baby was transferred to the neonatal ICU, where she remained for 3 months. The placenta was sent to histopathological analysis that evidenced the presence of vascularized tumor of $6 \times 2.5 \times 2 \mathrm{~cm}$ compatible with chorioangioma, as well as areas of bleeding and placental infarction, with signs of villous hypovascularisation. 


\section{E1219 - A CASE OF RHIZOMELIC CHONDRODYSPLASIA PUNCTATA TYPE I IN A MALE FETUS}

Yavuzkir S. ${ }^{1}$, Melekoglu . $^{2}$

Firat University School of Medicine, Obstetrics and Gynecology, Elazig-Turkey ${ }^{1}$

Inonu University School of Medicine, Obstetrics and Gynecology, Malatya-Turkey ${ }^{2}$

Chondrodysplasia punctata (CDP) is a rare autosomal recessive genetic disorder that is characterized by severely symmetric short limbs, abnormal ossification of the proximal extremities, congenital cataracts, ichthyosiform skin changes, microcephaly, and mental retardation. The incidence of this skeletal dysplasia is estimated at 0.9 per 100,000 live births and infant mortality is high within the first year of life. Antenatal sonographic diagnosis of the disease is usually based on the demonstration of the rhizomelic bone shortening and epiphyseal stippling. Although $75 \%$ of neonates with CDP have cataracts, prenatal sonographic diagnosis of fetal congenital cataracts has rarely reported. Herein, we aimed to report the antenatal ultrasonographic features of CPD case including congenital cataract and rhizomelic symmetric long bone shortening in the second trimester of pregnancy. A 28-yearold pregnant woman gravida 5 para 4, referred to our Prenatal Diagnosis and Treatment Unit due to dysmorphic face appearance in routine sonographic assessment during the 25th week of pregnancy. There was no abnormal characteristic in her medical history. Also, there was no pathologic finding in her physical examination and laboratory values. Obstetric ultrasonography revealed a fetus with bilateral pelvicaliectasis, bilateral short femur, and humerus, frontal bossing, microphthalmia, and cataract (Image-1). The patient and her partner were informed about the fetal status, and the option of invasive prenatal diagnosis (cordocentesis) was offered. The parents refused the invasive prenatal diagnostic procedure, and subsequent follow-up evaluations were performed at two weeks interval. Elective cesarean section was planned at 37 weeks of gestation due to intrauterine growth retardation after one course of betamethasone administration. APGAR score 7/8, $2350 \mathrm{~g}, 46 \mathrm{~cm}$ male infant was delivered by cesarean section. Physical examination revealed rhizomelic micromelia, bilateral cataracts, broad nasal bridge and long philtrum that suggest CDP syndrome (Image-2). The karyotype of the fetus was 46, XY. No unusual single nucleotide polymorphisms were detected. Whole exome analysis showed that the fetus was homozygous for the PEX7 mutation (c370_396 delGGTGAACAGCTTGTGGTGTCTGGCTCA pG124 S132del), which is compatible with rhizomelic CPD type I. In the second month of life, the baby died due to pulmonary infection and respiratory failure in intensive care unit. In conclusion, the detection of symmetric rhizomelic shortening and bilateral cataracts in antenatal ultrasonography in a fetus without a positive family history, the diagnosis of rhizomelic CPD should be considered in the differential diagnosis. 
E1223 - PRENATAL DIAGNOSIS OF DISTAL TRISOMY 17Q SYNDROME A CASE REPORT

Melekoglu R. ${ }^{1}$, Yilmaz E. ${ }^{1}$

Inonu University School of Medicine, Obstetrics and Gynecology, Malatya-Turkey ${ }^{1}$

The precise detection of the chromosomal abnormality is a crucial factor when evaluating the genotype-phenotype correlation in clinical cytogenetics. De novo and complex imbalanced chromosomal rearrangements often difficult to detect by using traditional cytogenetic techniques. Microarray comparative genomic hybridization (CGH) has recently been used to identify sub-microscopic chromosomal aberrations in fetal medicine especially in pregnant women complicated with fetal anomalies. Trisomy of the distal portion of long (q) arm of chromosome 17 is a rare condition and until now both de novo and inherited a few cases have been reported in the literature. The syndrome is characterized by microcephaly, mental retardation, intrauterine growth retardation, short and webbed neck, rhizomelia, polydactyly, short stature, and distinct facial features. In this report, we presented a fetus with antenatally diagnosed distal trisomy $17 q$ syndrome by the sonographic features detected in mid-trimester fetal anomaly scan and microarray CGH analysis. A 37 years old woman, gravida 2 para 1 , attended the Prenatal Diagnosis and Treatment department for the purpose of routine secondtrimester fetal ultrasonography scan at 22 weeks of pregnancy. The first-trimester screening test was within normal limits. Her obstetric and medical histories were unremarkable. She had no consanguinity with her spouse. Fetal ultrasonography scan revealed bilateral ventriculomegaly, vermian agenesis, bilateral polydactyly, flattened nose, bilateral short femur and humerus (Image-1). After the detailed counseling of the parents about the fetal malformation, fetal magnetic resonance imagining (MRG) and invasive prenatal diagnosis were planned. Fetal MRG showed corpus callosum hypoplasia and confirmed the diagnosis of vermian agenesis and bilateral ventriculomegaly. Microarray CGH analysis revealed distal trisomy 17q (17q21.32q25.3) syndrome. The option of termination of pregnancy was offered, which the parents refused, and subsequent follow-up scans were performed at two weekly intervals. Elective cesarean section was planned at 39 weeks of gestation due to the history of previous cesarean section. A male infant was delivered, Apgar scores were 6 at one minute and 8 at five minutes, birthweight was $2750 \mathrm{~g}$, the length was $48 \mathrm{~cm}$. On physical examination, he had polydactyly in both hands and feet that suggested distal trisomy $17 \mathrm{q}$ syndrome (Image-2). In the 17th day of life, the baby died due to respiratory failure in intensive care unit. The case is presented because of its rare occurrence and to provide a beneficial contribution to genetic counseling of parents in fetuses with similar antenatal ultrasonographic features. 
E1226 - A CASE OF ANTENATALLY DIAGNOSED AMBIGUOUS GENITALIA IN A FETUS IN MID TRIMESTER FETAL ANOMALY SCAN

Melekoglu R. $^{1}$, Cengiz M. ${ }^{1}$, Yilmaz E. ${ }^{1}$

Inonu University School of Medicine, Obstetrics and Gynecology, Malatya-Turkey ${ }^{1}$

Sexual differentiation is a complex process that leads to the formation of male or female internal and external genitalia. The interaction of sex chromosome, gene products that regulate the migration of germ cells and gonadal hormone production determine the fetal gender. Although fetal sex can be detected accurately from 12 weeks of gestation, typically the prenatal determination of fetal gender is performed by ultrasonographic examination of the external genitalia in the second trimester. While most of the sex development disorders are commonly isolated, the determination of associated congenital anomalies and evaluation of maternal and family history may contribute to define the origin of the disease. Laboratory studies including chromosome analysis, array comparative genomic hybridization analysis, and amniotic fluid hormone measurements are indicated when a suspect of sex development disorder occurs. The diagnosis of ambiguous genitalia in a fetus presents a challenge for both the clinician and the family. A multidisciplinary approach including perinatology, pediatric endocrinology, genetics, and psychology is essential for the management of the condition. We aimed to describe a case of antenatally diagnosed ambiguous genitalia in a fetus in midtrimester fetal anomaly scan. A 34 years old woman, gravida 2 para 1, attended the Prenatal Diagnosis and Treatment Unit for a routine second-trimester fetal anomaly scan at 22 weeks of pregnancy. The first-trimester screening test was within normal limits. Her obstetric and medical histories were unremarkable. She had no consanguinity with her spouse. Fetal ultrasonography scan revealed abnormal phallic structure (short shape) and bifid scrotum in the examination of fetal external genitalia. (Image-1) No associated anatomic anomalies were detected. After the detailed counseling of the parents about the sex development disorder and expected neonatal prognosis with a pediatric endocrinologist; the invasive prenatal diagnosis was offered for further evaluation. They refused the invasive test, and subsequent pregnancy follow-up was performed routinely. APGAR score 9/10, $3450 \mathrm{~g}, 49 \mathrm{~cm}$ infant with ambiguous genitalia was delivered by vaginal delivery in 40 weeks of gestation. Physical examination of neonate revealed ambiguous genitalia, and there was no any other pathologic finding (Image2). The laboratory tests including biochemical (glucose, electrolytes) and hormone measurements (FSH, LH, DHEAS, free and total testosterone, ACTH, cortisol) are all detected in the normal range. The karyotype of the fetus was showed no deletion in SRY region and 46, XY/47, XXY mosaicism was found (20\% of cells were detected XXY and $80 \%$ of cells determined XY). Neonate was discharged at postnatal 10th day and at the time of writing, the baby showed normal growth and no additional intervention was planned by the Paediatric Endocrine Clinic for the present. In conclusion, ambiguous genitalia can be diagnosed antenatally by the ultrasonographic evaluation of fetal external genitalia for the characteristic ultrasonographic findings. The determination of associated abnormalities, offering invasive prenatal diagnosis and counseling with a multidisciplinary team is essential for the management of this sex development disorder. 


\section{E1229 - NASOPALPEBRAL LIPOMA COLOBOMA SYNDROME REPORT OF A CASE}

Melekoglu N. ${ }^{1}$, Melekoglu R. ${ }^{2}$

Malatya Education and Training Hospital, Neonatology, Malatya-Turkey ${ }^{1}$

Inonu University School of Medicine, Obstetrics and Gynecology, Malatya-Turkey ${ }^{2}$

Nasopalpebral lipoma-coloboma syndrome (OMIM 167730) is an extremely rare autosomal dominant condition characterized by bilateral nasopalpebral lipomas, bilateral upper and lower eyelid colobomata, a broad forehead, telecanthus, and maxillary hypoplasia. The etiology is unknown, but it has been hypothesized that a migration defect of the neural crest cells leads to abnormal development of the facial anatomy. We aimed to present a prenatally diagnosed newborn with nasopalpebral lipoma-coloboma syndrome due to facial dysmorphic appearance and hypertelorism. A 33-year-old pregnant woman, gravida 1 para 0 , was referred to a specialized perinatologist for second-trimester detailed ultrasonographic examination because of hypertelorism. Her medical and obstetric history was unremarkable, but fetus's father had a facial reconstructive operation history due to hypertelorism at childhood. Parents were non-consanguineous. Fetal ultrasonography scan revealed ordinary findings except for facial dysmorphic appearance due to the narrowed nasopalpebral region and marked telecanthus. These findings were evaluated with family history, and genetic counseling was requested. At term, a $3320 \mathrm{~g}, 50 \mathrm{~cm}$ female baby was delivered by cesarean section. On physical examination, she had a bilateral symmetrical accumulation of subcutaneous tissue in the nasopalpebral region, extending to the forehead and causing marked telecanthus. Symmetrical colobomata located at the junction of the inner and middle thirds of upper lids, epiphora, and absence of medial eyelashes were disclosed. There were no other systemic anomalies. Genetic counseling recommended to the family, and with these clinical features, the diagnosis of was nasopalpebral lipoma-coloboma syndrome confirmed. Genetic studies are ongoing on the identification of the disorder. The case is presented because it is rarity. A multidisciplinary approach is required because of ophthalmological manifestations, and cosmetic surgery may be necessary. 


\section{E1234 - APLASIA CUTIS CONGENITA CASE REPORT}

Dos Santos G. ${ }^{1}$, Martins M. ${ }^{1}$, Carvalho M. ${ }^{1}$, Pimentel L. ${ }^{1}$, Silveira S. ${ }^{2}$, Gieburowski A. ${ }^{1}$, Feuerschuette O. ${ }^{3}$, Cantarelli M. ${ }^{1}$, Martins A. ${ }^{1}$, Trapani Jr A. ${ }^{3}$

Hospital Universitário/Ufsc, Obstetrics, Florianópolis-Brazil ${ }^{1}$

Hospital Universitário/ Ufsc And Maternidade Carmela Dutra, Obstetrics, FlorianópolisBrazil $^{2}$

Hospital Universitário/Ufsc and Unisul, Obstetrics, Florianópolis-Brazil ${ }^{3}$

Aplasia cutis congenita (ACC) is a rare disease characterized by incomplete skin formation. Clinical presentation at birth is variable, depending upon the time of occurrence, tissue layers involved, and degree of healing in utero. It can affect any part of the body but in $85 \%$ of cases it is located on the scalp. Although in most cases ACC is a benign isolated defect, it may be associated with a number of genetic syndromes and congenital anomalies. The exact pathophysiology of ACC is unclear. Proposed mechanisms include intrauterine trauma, vascular compromise, infection, and medications such as methimazole, misoprostol, and valproic acid.

\section{Case}

A 37-year-old black pregnant woman, G4P2A1, was referred to our prenatal care due to gestational diabetes. She was managed with healthy diet and physical activity and had an adequate glycemic control throughout pregnancy. She was admitted to our hospital at gestational age of $29+3$ weeks with preterm prelabor rupture of membranes. Ultrasound scan showed adequate fetal growth and oligohydramnios. There was no evidence of intrauterine infection. She was managed expectantly until 34 weeks of gestation when her labor was induced with misoprostol and oxytocin. She gave birth to a baby boy, Apgar score 9/9, weighing 2025g. At birth, a cutaneous malformation was observed crossing the insertion of the umbilical cord, measuring 6 to $8 \mathrm{~cm}$, yellowish and with softened consistency. No other congenital anomaly was observed. There were no changes in placenta or umbilical cord and no reports of cutaneous changes in relatives. A pediatric dermatologist confirmed the diagnosis of ACC. The lesion was treated with silver sulfadiazine cream and nonadherent dressings. After eight days the newborn was discharged home. His mother was oriented to continue wound care. Two weeks later, his lesion was almost totally healed.

Suspicious of AAC by antenatal ultrasound examinations is difficult. Diagnostic is made by physical findings and biopsy of the lesion after birth. Clinical course and severity of ACC varies depending upon the size of the defect and the tissues involved. Small lesions have a good prognosis and usually heal spontaneously over weeks to months. Large lesion may require surgical repair. Associated anomalies may affect the prognosis. Because it is a rare disease, there is a difficulty in standardization of management. Treatment with silver sulfadiazine seems to be effective in healing superficial wound and ensuring an evolution without sequelae. 


\section{E1279 - THE DETECTION OF TWO COPIES OF SURVIVAL MOTOR NEURON GENE 1 IN ARTHROGRYPOSIS MULTIPLEX CONGENITA-SPINAL MUSCULAR ATROPHY ASSOCIATION A CASE REPORT}

Melekoglu R. ${ }^{1}$, Yilmaz E. ${ }^{1}$

Inonu University School of Medicine, Obstetrics and Gynecology, Malatya-Turkey ${ }^{1}$

Arthrogryposis multiplex congenita (AMC) is a rare sequence of congenital joint fixation disorder with a reported incidence of 1/3000 live births. Neurogenic, or muscular disorders, connective tissue abnormalities, intrauterine compression due to severe oligohydramnios, vascular compromise, or teratogenic factors could be the primary cause of the ultrasonographic findings. While neuronal degeneration occurs in the anterior horn of the central nervous system, it has been proposed that the neurogenic origin of AMC could be associated with acute spinal muscular atrophy (SMA)(SMA type I). The diagnosis of AMCSMA association is one of the rarest forms of spinal muscular atrophy that have been described as an atypical form of infantile SMA. We aimed to present a case of AMC-SMA association because of its rare occurrence and postnatally detection on the genetic basis. A 31year-old gravida 5 parity 3 pregnant woman with a history of one first-trimester fetal loss was referred to our Prenatal Diagnosis and Treatment Unit due to severe polyhydramnios and micrognathia during the 34th week of gestation. The parents had documented second-degree consanguinity but both did not have any significant family history. Also, there were no pathologic findings in physical examination and laboratory values of pregnant. Obstetric ultrasonography revealed that the biometric measurements of the fetus were lower than 5 percentile according to the gestational age and the placenta was observed in the posterior wall of the uterus with normal appearance. The amniotic fluid index was determined $61.1 \mathrm{~cm}$, suggesting severe polyhydramnios and the fetus was in the breech position. Decreased fetal movements, fixed extension deformities in both lower and upper extremities, mineralization defect in long bones, small chest, subcutaneous edema, and dysmorphic features including hypotelorism, micrognathia were noted in the fetal anatomical assessment. (Image-1) The diagnosis of AMC was considered and the patient and her partner were informed about the fetal status. Emergency cesarean section was planned due to fetal distress (reversed diastolic flow in the umbilical artery ), and APGAR scores 3/4, $2073 \mathrm{~g}, 47 \mathrm{~cm}$ male infant was delivered. In physical examination, he had micrognathia, severe edema, marked hypotonia, right humerus fracture, and multiple contractures in both extremities. (Image-2). The infant was intubated due to poor respiratory effort. Genetic tests including STR fragment analysis and Multiplex Ligation-dependent Probe Amplification test determined two copies of survival motor neuron gene-1 (SMN-1) gene. The infant continued to require ventilatory support and had no spontaneous respiratory movements during the follow-up period, and the baby died due to respiratory failure in intensive care unit in the 28th day of life. In conclusion, the SMN gene should be carefully investigated in fetus diagnosed with AMC for the evidence of spinal cord involvement. While AMC of neurogenic origin remains a genetically heterogeneous condition SMN gene analysis will make the diagnosis easier in the AMC-SMA association, thus contributing to elucidate the classification of AMC. Not only SMN gene deletion analysis also SMN gene dosage analysis in negative cases for the detection of SMN copies could be of help in the genetic counseling of this association. 


\section{E1280 - A CASE OF ALKURAYA KUCINSKAS SYNDROME DIAGNOSED IN PRENATAL PERIOD}

Özalp M. ${ }^{1}$, Şal H. ${ }^{2}$, Özbay G. ${ }^{2}$, Cömert E. ${ }^{2}$, Osmanağaoğlu M. ${ }^{1}$

KTU, Perinatology, Trabzon-Turkey ${ }^{1}$ KTU, Obstetrics and Gynecology, Trabzon-Turkey ${ }^{2}$

\section{Objective}

Alkuraya-Kucinskassendroma (AKS) is an autosomal recessive, serious neurodevelopmental disorder caused by a homozygous or compound heterozygote mutation in the KIAA1109 gene in the 4q27 chromosome. It is characterized by arthrogryposis and brain abnormalities associated with minimal development of brain parenchyma. It is also characterized by developmental delay. In this article, we wanted to present a case with Alkuraya Kucinskas syndrome.

\section{Case}

27 years old G2P0A1, LMP 23 weeks 2 days pregnant, she was referred because of hydrocephalus and clenched hand from external center to our perinatology clinic. Apart from her second degree relative marriage, there was no feature in her story. In the ultrasonographic examination, fetus was compatible with 18 weeks and 5 days. Intrauterine growth retardation, hydrocephalus, corpus callosum agenesis, micrognathia, clubfoot in the right lower extremity, bilateral clenched hand were observed. Amniocentesis was performed, the result was reported as arr $(1-22, \mathrm{X}) \mathrm{x} 2$. The family was offered a termination option. The family did not accept termination. While 36 weeks and 5 days of gestation according to SAT, premature rupture of membranes and fetal condition due to unreliable fetal condition, emergency cesarean delivery was performed. APGAR score 1.-5. minute, 0. The baby was ex. Fetal autopsy was recommended to the family and the family accepted. In autopsy of the fetus, $2475 \mathrm{~g}, 47 \mathrm{~cm}$ in height, sex in the external examination of the female fetus micrognathia, retrognathia, bilateral clenched hand, bilateral clubfoot were observed. No abnormality was observed in the abdominal organs. In the central nervous system examination, $225 \mathrm{~g}$ of the brain, giral pattern flattened, ventriculomegaly were observed, midbrain, thalamus and hippocampus (temporal lobe) could not be selected. Lissencephaly, hydrocephalus, corpus callosum agenesis were evaluated. This case, which had hydrocephalus and arthrogryposis findings, was evaluated as ACS.

\section{Discussion}

Alkuraya Kucinskas syndrome is a fatal disease with hydrocephalus and arthrogryposis. The incidence is unknown. In the clinical course, cerebellar hypoplasia with cerebral atrophy, lissencephaly, mild or severe ventriculomegaly and brain stem dysgenesis may be seen. Other diseases associated with arthrogryposis should be reviewed in the differential diagnosis. Most of the affected individuals die in the antenatal period or immediately after birth. The surviving patients were followed by mental retardation and seizures. In the KIAA1109 gene there are 14 mutations identified as homozygous or compound heterozygous. Gueneau et al. found that the destruction of the KIAA1109 gene in the zebrafish by CRISPR technology resulted in increased frequency of hydrocephalus or other head defects and increased body curvature compared to control groups.

\section{Conclusion}

Alkuraya Kucinskas syndrome is a neurodevelopmental disease that can be diagnosed easily in the first trimester. Families should be informed about the prognosis of pregnancy and the termination option should be explained. AKS should be considered in fetuses with brain parenchymal defect and skeletal anomaly and genetic examination should be recommended. 


\section{E1301 - ANTENATAL DIAGNOSIS OF A RARE CASE OF EVENTRATION OF THE DIAPHRAGM HIGH INDEX OF SUSPICION}

$\underline{\text { Sallout B. }^{1}}{ }^{\text {, Al Shebli D. }}{ }^{1}$, Al Baqawi B. ${ }^{1}$

King Fahad Medical City, Maternal Fetal Medicine, Riyadh-Saudi Arabia ${ }^{1}$

Congenital diaphragmatic eventration is rare congenital anomaly. A case of diaphragmatic hernia was evaluated carefully with high suspicion of diaphragmatic eventration. 26 years, primigravida with normal first trimester scan. Anatomy scan showed; multicystic mass on the left side of the chest, heart and stomach were in normal position, diagnosed as congenital pulmonary adenoid malformation (CPAM). Follow up scan at 25 weeks, showed the same chest cystic mass with absent stomach. The diagnosis was changed to congenital diaphragmatic hernia. Fetal MRI at 26 weeks; showed a multicystic lesion seen in the lower thorax $(5 \times 2.2 \mathrm{~cm})$, no definitive communication with abdominal cavity. The findings are nonspecific, may indicate diaphragmatic hernia or CPAM.

As the scans and MRI were not conclusive, a comprehensive scan at 36 weeks was performed, the left kidney was in a higher position under the left hemidiaphragm, and heart and stomach were in normal position. The case was diagnosed as diaphragmatic eventration. Pregnancy progressed uneventfully and patient delivered at 39 weeks. The neonate was intubated and admitted to NICU. Chest and abdomen X-RAY shows herniated bowel loops across the diaphragm to the thorax. At day two, chest CT with intravenous contrast showed no lung mass, no pleural effusion and the left hemidiaphragm was elevated. Diagnosed as left diaphragmatic eventration with elevation of small bowel loops and left kidney underneath the elevated left hemidiaphragm.

The neonate was extubated after 3 days, pediatric surgery advised no need to interfere. The neonate was discharged in day 17 on stable condition with follow up appointment with neonatology and pediatric surgery.

Eventration of the diaphragm is abnormal elevation of one leaf of an intact diaphragm as a result of paralysis or atrophy of varying degrees of muscle fibers. Eventration of the diaphragm is a rare anomaly, occurring in $0.002 \%$ of live births. The prenatal differentiation between congenital diaphragmatic eventration and congenital diaphragmatic hernia by sonography is very difficult because the 2 disease have similar sonographic appearances. Antenatal diagnosis of diaphragmatic eventration is vital as the neonate relies upon the diaphragm for the normal respiration.

The antenatal diagnosis of eventration of the diaphragm is unusual and need high index of suspension as the features can be easily interpreted as diaphragmatic hernia. Diaphragmatic eventration was suspected based on several features:

1- The appearance of the chest lesion was inconsistent in different scans

2- The absence of cardiac displacement as in cases of diaphragmatic hernia

3- The presence of normal right lung head ratio

4- The presence of normal stomach and absent stomach in different scans.

5- Normal amniotic fluids which is unusual in case of diaphragmatic hernia

6- The intact diaphragm 


\section{E1302 - PRENATAL DIAGNOSIS OF ADRENAL NEUROBLASTOMA} DIFFERENTIAL DIAGNOSIS AND MANAGEMENT

$\underline{\text { Psarris A. }}^{1}$, Sindos M. ${ }^{1}$, Theodora M. ${ }^{1}$, Antsaklis P. ${ }^{1}$, Psarakis A. ${ }^{1}$, Kataras T. ${ }^{1}$, Loutradis D. $^{1}$, Daskalakis G. ${ }^{1}$

"Alexandra" Maternity Hospital, National and Kapodistrian University of Athens, $1^{\text {st }}$

Department of Obstetrics and Gynecology, Athens-Greece ${ }^{1}$

Neuroblastoma is the most common extracranial solid tumor of infancy with an incidence rate of 58 per million infants younger than one year old. Neuroblastomas originate from the neural crest during fetal development and may arise from the sympathetic ganglia or the adrenal medulla. We present the case of a cystic neuroblastoma of the left adrenal gland that was detected prenatally during a routine antenatal visit.

A 27-year-old pregnant woman visited the outpatient clinic of Alexandra maternity hospital during the third trimester of her pregnancy. The woman was Gravida 2 Para 0 Abortus 1 . The gestational age of the pregnancy was 38 weeks and 2 days. The woman's past medical history was uneventful. During her pregnancy she was subjected to prenatal testing including first trimester scan, anomaly scan, 3rd trimester - doppler scan without any abnormal findings. During our initial ultrasound examination, we detected one fetus in occiput anterior position. All growth parameters (Biparietal Diameter, Head Circumference, Abdominal Circumference, Femur Length, Estimated Fetal Weight) and the Amniotic Fluid Index were within the normal range for the gestational age. During the scan a well circumscribed mass was detected over the left kidney (Figure 1). The mass appeared to have both cystic and solid areas and appeared to originate from the left adrenal gland (Figure 2a). The anatomy of the left kidney was normal. The mass measured $4.49 \times 3.95 \mathrm{~cm}$ (Figure 2b). Doppler evaluation did not reveal increased blood flow in the mass (Figure 3). The unilateral kidney and unilateral adrenal gland appeared normal (Figure 4). Extensive ultrasound examination of the fetus revealed no other anomalies. After careful consideration the diagnosis of cystic adrenal neuroblastoma was made. Taking into consideration the gestational age of the pregnancy, induction of labor was programmed at 39+1 weeks, resulting in the birth of a male neonate with APGAR score 9 in the first minute. Transabdominal ultrasound examination confirmed the diagnosis. The neonate was transported to the pediatric oncology department where it was subjected to abdominal MRI and serum and urine VMA and HVA tests which verified the diagnosis of neuroblastoma.

Early detection of neuroblastomas is very important since treatment of low stage disease results in favorable oncologic outcomes (for instance surgery is curative for neonates with small adrenal masses). Detection of antenatal fetal neuroblastoma was described for the first time in1983 by Fenart et al. Since then, the evolution of imaging technics has allowed earlier detection of fetal tumors. Differential diagnosis of fetal abdominal tumors is based on tumor characteristics, location, vasculature and the gestational age at detection. Fetal suprarenal tumors include neuroblastoma of the adrenal gland, extra lobular pulmonary sequestration, adrenal hematoma, adrenal abscess, adrenal nodular hyperplasia, adrenal cyst, bronchogenic cyst and adrenal carcinoma. Evaluation by a maternal - fetal medicine specialist is essential for the proper management of the case. 


\section{E1303 - A TREACHER COLLINS CASE INVESTIGATION CHALLENGES IN INTERPRETING GENETIC ANALYSIS RESULTS}

$\underline{\text { Psarris A. }}{ }^{1}$, Sindos M. ${ }^{1}$, Psarakis A. ${ }^{1}$, Theodora M. ${ }^{1}$, Antsaklis P. ${ }^{1}$, Pampanos A. ${ }^{2}$, Loutradis D. ${ }^{1}$, Daskalakis G. ${ }^{1}$

"Alexandra" Maternity Hospital, National and Kapodistrian University of Athens, $1^{\text {st }}$

Department of Obstetrics and Gynecology, Athens-Greece ${ }^{1}$

"Alexandra" Maternity Hospital, Genetics Department, Athens- ${ }^{2}$

Treacher-Collins Syndrome (TCS) (also known as mandibulofacial dysostosis) is a malformation of the craniofacial development. TCS 1 and TCS 2 are autosomal dominant disorders with a variable degree of penetrance, while TCS 3 is autosomal recessive and about $10 \%$ of the TCS patients have an unknown underlying genetic defect. Treacher-Collins Syndrome affects about 1:50.000 live births. The cause of the disease is the presence of mutations in the TCOF1, POLR1D $\kappa \alpha$ POLR1C genes. Treacher Collins phenotype includes zygomatic hypoplasia, antimongoloid slant of the eyes, eyelid notches, class 2 malocclusion, external ear abnormalities, hearing loss, cleft lip and choanal atresia. We present a case of a 36-year-old pregnant woman, who visited our outpatient maternity clinic for prenatal screening. She was Gravida 2 Para 1 Abortus 0. She was carrying dichorionic diamniotic twins and the gestational age of the pregnancy was 11 weeks. She reported a stillbirth with a diagnosis of Treacher-Collins Syndrome based on the phenotype of the neonate, without molecular analysis confirmation. After subjecting her to molecular analysis she was found heterozygous for the single nucleotide polymorphism (SNP) c.1232A $>$ G (p.Gin411Arg) of the TCOF1 gene. A small zygomatic hypoplasia of the patient was attributed to this mutation. Following her diagnosis, she was subjected to chorionic villus sampling and molecular analysis which was negative for the c.1232A $>$ G (p.Gin411Arg) SNP of the TCOF1 gene in both fetuses. Despite the negative result, close ultrasound follow-up of the pregnancy was advised as there was a discrepancy regarding the characterization of c.1232A $>$ G (p.Gin411Arg) in different gene databases. After reviewing Clinvar, HGMD, LOVD, Clinvitae, Decipher, gnomAD and Bravo Topmed for said SNP, it was not considered a pathologic polymorphism in $6 / 7$ but it was considered to be a pathologic polymorphism in 1/7. At 21 weeks of gestation the anomaly scan detected cleft palate and zygomatic hypoplasia of fetus A. Further testing of the chorionic villi samples with high resolution chromosomal microarray was ordered and a $462 \mathrm{~kb}$ deletion was detected in the $5 \mathrm{q} 32$ (chr5:149,283,356_149,745,109) chromosomal region in both fetuses. This region included 9 documented genes, one of which is TCOF1. After thorough genetic counselling the woman decided to terminate the pregnancy.

The presence of mutations with unknown clinical value and the discordance in characterization of mutations as pathologic or not between different databases creates uncertainties regarding genetic counselling. Hence attributing phenotypic characteristics to SNPs with uncertain clinical significance may often be misleading. Furthermore, in case of stillbirths with suspected congenital anomalies or genetic syndromes it is imperative that they are subjected to molecular analysis before any diagnosis are made. 


\section{E1317 - CONGENITAL HIGH AIRWAYS OBSTRUCTION SYNDROME (CHAOS) CASE REPORT OF TWO WOMEN WITHOUT CLASSICAL ULTRASOUND FINDING}

Ahmeed S. ${ }^{1}$, Alafasy H. ${ }^{1}$, Sallout B. ${ }^{1}$, Albaqawi B. ${ }^{1}$

King Fahad Medical City, Maternal-fetal Medicine, Riyadh-Saudi Arabia ${ }^{1}$

Congenital high airways obstruction syndrome (CHAOS) is a rare fetal anomaly. The underlying cause is congenital complete or incomplete obstruction of fetal upper airway tracts during embryogenesis, resulting in a spectrum of characteristic radiological feature diagnosed prenatally by ultrasound at time of routine anatomy scan. In many cases the diagnosis will be delayed to postnatal period at time of delivery when the newborn fail to open his/ her airway immediately after delivery.

We report our experience of two cases of CHAOS diagnosed only in the postpartum examination when there were no characteristic findings on detailed ultrasound examination.

\section{Case 1}

A 24-years old, was referred early at 11 weeks for dating ultrasound scan. Anatomy scan was showing normal appearance of 4 chamber view and normal diaphragm shape. During her antenatal follow up, the fetus was diagnosed to have absent right kidney, dilated bowel loop and he developed fetal growth restriction (FGR). She underwent cesarean section for breech presentation and FGR. The baby delivered alive, has dysmorphic features with abnormal microotia, micrognathesia and high arch palate and bifid tongue, trial of intubation failed as no laryngeal opening on examination. Infant died post CPR for 38 minutes after birth. Neonatal whole exome sequencing revealed, Fraser syndrome (FRAS1 gene) but with variant of unknown significance.

\section{Case 2}

A 33-years old was referred early at 15 week and anatomy scan was normal apart from echogenic lungs but normal cardiac axis, mediastinum and diaphragm curvature. Growth scan was done at 34 weeks and showed polyhydramnios with AFI of $38 \mathrm{~cm}$. At time of delivery the baby was not crying and NICU team failed to intubate hem, bronchoscopy examination showed subglottic laryngeal atresia, tracheostomy was done and the baby died after 30 min of CPR. Neonatal blood was obtained for whole exome sequencing and the results showed that the baby was homozygous for, Fraser syndrome (FRAS1 gene) ch.4 c.2917T>G, p. Cys 973 Gly. The result was reported as a variant of unknown significance.

\section{Conclusion}

The current diagnostic criteria are not applicable for all the cases and physicians need to have high index of suspicion for the diagnosis when the lungs got to be echogenic in the ultrasound. CHAOS can be part of FRASER syndrome but we are reporting a new variant that has not been identified to be of clinical significance till the mean time. 
E1327 - PRENATAL SONOGRAPHIC DIAGNOSIS OF HARLEQUIN ICHTHYOSIS A CASE REPORT

Erol A. ${ }^{1}$, Öcal D. ${ }^{1}$, Halıcı Öztürk F. ${ }^{1}$, Yakut K. ${ }^{1}$, Kırbaş A. ${ }^{1}$, Çelen Ş. ${ }^{1}$

University Of Health Sciences, Zekai Tahir Burak Women's Health Education and Research

Hospital, Perinatology, Ankara-Turkey ${ }^{1}$

\section{Aim}

We report as a case of successful prenatal diagnosis of HI during the third trimester sonographic examination in a related couple with history of the disease.

\section{Case}

A 30-year-old gravida 3 para 2 woman, at the 32th week of gestation, was referred to our perinatology department for second opinion ultrasound (US). The couple was third degree consanguine and their first baby died on the fourth day of life, with an undiagnosed skin disease. Her routine aneuploidy screenings and pregnancy follow-ups were uneventful but she did not have a second trimester ultrasonographic examination for abnormality screening. On 2-dimensional (2D) and 4-dimensional (4D) US; facial dysmorphism with distorsion of the lips (eclabion), conjunctival protrusion associated with severe chemosis (ectropion), skin fissures, dense floating particles in the amniotic fluid, short digits, flattened nose and ears, severe edema on the dorsal surfaces of hands and feet were detected. These findings were considered as sonographic features of HI. (Figure 1-4). After counselling about the disease exactly, the patient did not accept any invasive procedures becouse of the risks. A male infant with HI (birth weight, 3190 gr) was delivered by cesarean section for breech presentation on active labor at 39 weeks of gestation. Sonographic signs were confirmed postnatally (Figure 5-6). Parenteral nutrition, hydration, antibiotherapy and skin care treatment were given to the infant in neonatal intensive care unit but he died due to sepsis on postpartum seventh day.

\section{Conclusion}

HI is a severe disorder of keratinization caused by mutations in the ABCA12 gene with autosomal recessive inheritance and related marriages could be a risk factor. The mutations lead to defective lipid transportation which negatively affects the correct development and function of the skin. The main phenotypic features include dry scaly fish-like skin, consisting of hyperkeratosis with erythematous fissures between thick yellowish armor-like plaques involving the entire body surface, ectropion and eclabium, flattened nose, malformed ears, abnormally fixed limbs and fingers, and toes in rigid flexion (mitten-like hands) due to the inability of the skin to expand. Even with intensive care (including treatment with retinoidsetratinate, acitretin), prognosis is poor and most neonates die shortly after delivery due to infection, heat loss, dehydration, electrolytic disturbances (eg, hypernatremia), or respiratory distress. Fetoscopic or US guided fetal skin biopsies are generally preferred for prenatal diagnosis. In conclusion US markers for HI should be kept in mind, particularly for early and accurate antenatal diagnosis of this devastating condition and detailed counselling should be given to the parents. 


\author{
E1356 - TRISOMY 18 CASE DIAGNOSED WITH CLENCHED HAND AND NT \\ THICKNESS IN THE FIRST TRIMESTER A CASE REPORT \\ Demir B. ${ }^{1}$, Demir S. ${ }^{2}$, Toktas I. ${ }^{1}$, Silan F. ${ }^{3}$, Ozdemir O. ${ }^{3}$ \\ Çanakkale Onsekiz Mart University,, Department of Obstetrics And Gynecology, Çanakkale,- \\ Turkey $^{1}$ \\ Dokuz Eylul University School of Medicine, Division Of Perinatology, Department of \\ Obstetrics and Gynecology, Izmir-Turkey ${ }^{2}$ \\ Çanakkale Onsekiz Mart University, Department of Medical Genetics,, Çanakkale,-Turkey ${ }^{3}$
}

\begin{abstract}
Aim
To present a case diagnosed with trisomy 18, whose chorionic villus sampling was performed due to clenched hand and increased NT thickness in the first trimester.

\section{Case}

The 41-year-old G3 P2 A1 L1 patient was referred to our perinatology outpatient clinic due to increased thickness of the NT, with a 13 weeks 3 day pregnancy according to her last menstrual period. In the detailed fetal examination of the patient, CRL was consistent with 11 weeks 5 days, NT: $3.4 \mathrm{~mm}$ and the fluid accumulated in the back of the neck was observed to envelop the whole body like a membrane. Flexion deformity and clenched hand appearance were observed in both wrists. The patient was informed about prenatal genetic diagnosis and chorionic villus sampling was performed with the consent of the patient. CVS FISH result was found as Trisomy 18. The patient was admitted for medical abortion for 14 weeks and 5 days of gestation, there was no fetal cardiac activity in the examination. Medical abortion was performed.
\end{abstract}

\title{
Conclusion
}

Trisomy 18 (Edwards' syndrome) is one of the rare genetic anomalies, but the most common chromosomal disorder after trisomy 21 . The incidence rate was reported as $3 / 10,000$ in newborns. The risk of trisomy 18 increases with maternal age but decreases with advanced gestational age. NT is the sonographic view of the accumulation of subcutaneous fluid, collected under the skin behind the fetal neck in the first-trimester of pregnancy. This term is used regardless of whether fluid accumulation exists only on the neck or throughout the body. The relationship of NT with the frequency of chromosomal disorders and other anomalies is not related to its appearance, but with its thickness. For trisomy 18, fetal loss rate is approximately $80 \%$ between 12 and 40 weeks. In patients with increased NT thickness and clenched hand findings in the first trimester, Trisomy 18 should be considered in the differential diagnosis and the family should be informed about the preinvasive diagnostic methods and fetal prognosis. 
E1419 - THE ROLE OF ULTRASOUND SCREENING IN DETECTING SKELETAL DYSPLASIAS IN SECOND TRIMESTER - THE CASE OF ACHONDROPLASIA

Neonakis S. ${ }^{1}$, Velegrakis A. ${ }^{2}$, More E. ${ }^{3}$, Papadopoulou E. ${ }^{4}$, Sifakis S. ${ }^{5}$

University of Crete, Department of Obstetrics and Gynecology, Heraklion-Greece ${ }^{1}$

Venizeleio General Hospital, Department of Obstetrics and Gynecology, Heraklion-Greece ${ }^{2}$

General Hospital of Rethimnon, Department of Obstetrics and Gynecology, Rethimnon

Greece $^{3}$

University of Crete, Department of Pediatrics, Heraklion-Greece ${ }^{4}$ Mitera Maternity

Hospital, Heraklion-Greece ${ }^{5}$

\section{Objective}

Achondroplasia is the most common osteochondrodysplasia and the most common non-lethal skeletal dysplasia, with an estimated prevalence between 1 in 10,000 to 30,000 births. It is an autosomal dominant disorder, mainly caused by mutations in the fibroblast growth factor 3 receptor (FGFR3) gene. However, most cases are sporadic and are attributed to de novo mutations. The differential diagnosis, in cases with suspicious sonographic findings, includes hypochondroplasia, lethal dysplasia and homozygous achrondroplasia.

We present 3 cases of achondroplasia that were diagnosed during routine ultrasound screening in the second trimester of pregnancy in the absence of any family history, and discuss the role of ultrasound as a screening tool for detecting this rare genetic disorder, as well as the various aspects of a comprehensive parental counseling, once the diagnosis has been confirmed by amniocentesis.

\section{Methods}

Detailed scan of fetal anatomy at 22-23 weeks of gestation, performed by a specialist in a Fetal-Medicine referral unit, raised the suspicion for this skeletal abnormality, due to findings such as shortening of long bones, brachydactyly and macrocephaly. Diagnosis was confirmed by amniocentesis.

\section{Results}

In all cases, molecular karyotype revealed the characteristic FGFR3 gene mutation and confirmed the diagnosis of achondroplasia. Subsequently, parents received extensive counseling by a fetal-maternal medicine specialist, as well as by a pediatrician with expertise in congenital abnormalities and genetic disorders, regarding the prognosis, the clinical presentation, the pattern of inheritance and the risk of recurrence in future pregnancies, the treatment options available after birth, and the need for genetic parental testing. Eventually, all 3 couples decided to terminate pregnancy.

\section{Conclusion}

Prenatal diagnosis of achondroplasia is often missed until 20 weeks of gestation, especially if there is no family history, because the characteristic sonographic finding of shortening of long bones and macrocephaly, which is the hallmark of dwarfism after birth, is not usually obvious earlier. Further investigation and genetic testing is needed to differentiate between other skeletal dysplasias. In all cases, subsequent thorough counseling is essential, as the management of pregnancy, in terms of termination, poses ethical dilemmas to the parents due to relatively advanced gestation and to the fact that mental status of the affected offspring is usually normal. 


\title{
Obstetrics - Intrapartum ultrasonography
}

\author{
E1057 - ULTRASONOGRAPHIC FINDINGS AND CLINICAL CHARACTERISTICS \\ OF VASA PREVIA A REPORT OF 8 CASES \\ Kamijo K. ${ }^{1}$, Ando H. ${ }^{1}$, Yamada S. ${ }^{1}$, Fuseya C. ${ }^{1}$, Kikuchi N. ${ }^{1}$, Ohira S. ${ }^{1}$, Kanai M. ${ }^{2}$, \\ Shiozawa T. ${ }^{1}$ \\ Shinshu University School of Medicin, Obstetrics and Gynecology, Matsumoto-Japan ${ }^{1}$ \\ Shinshu University School of Health Sciences, Family and Child Nursing, and Midwifery, \\ Matsumoto-Japan ${ }^{2}$
}

\section{Objective}

Vasa previa is defined as a condition in which fetal blood vessel(s) locate on the membranes within $2 \mathrm{~cm}$ from the internal cervical os. A growing rate of the incidence has been reported, possibly due to prevalence of assisted reproductive technology. Vasa previa is classified into two categories: Type I) vessels that connect a velamentous umbilical cord to the placenta. Type II) vessels connect the lobes of a bilobed placenta or the placenta and a succenturiate lobe. However, several studies reported the cases which cannot be classified into these two types. In this study, we aimed to investigate the relationship between the disease types and clinical characteristics of vasa previa.

\section{Methods}

A retrospective descriptive study of all vasa previa cases that were managed in Shinshu University Hospital between 2010 and 2018 was performed. Data of maternal background, ultrasound findings of the cervix, placenta, and covering vessels, as well as obstetric and neonatal outcomes were reviewed and analyzed. This study was approved by the Ethics Committee of Shinshu University, School of Medicine.

\section{Results}

There were 8 cases of vasa previa among 7579 deliveries. All cases were diagnosed antenatally and delivered by cesarean section before the onset of labor. Six cases were nulliparous, and 2 were multiparous. Four had a history of assisted reproductive technology. The maternal age at referral was $35.1 \pm 3.7$ years (mean \pm SD). The gestational age at the time of diagnosis of vasa previa and delivery was $31 \pm 5.6$ weeks (mean \pm SD) and $36 \pm 2.0$ weeks (mean $\pm \mathrm{SD}$ ), respectively. Six (75\%) cases were associated with the low-lying placenta. Five (62.5\%) were classified as Type I, but there was no Type II case. The remaining 3 (37.5\%) cases could not be classified into two categories because the aberrant fetal vessel located out from the placenta which had normal cord insertion and no multipartite variant (unclassified, or "Type III”). The maternal and neonatal outcomes were acceptable in all cases. In one case, vasa previa was not detected by routine screening ultrasonography and color Doppler because the aberrant vein covered the internal os through posterior-anterior direction, which was not clearly visualized as a vasculature. The color Doppler image also resembled with flowing amniotic fluid, which could be distinguished by observing the steady flow with pulse-doppler imaging.

\section{Conclusions}

In our study, a considerable number of cases was not matched to the typical classification. Obstetricians should be aware of such cases that associate with no abnormal cord insertion nor multipartite placenta. Caution should be paid to vasa previa of with aberrant venous vessel because these cases may not have a clear image of vasculature and may have nonpulsatile flow on color Doppler imaging. A pulse-doppler imaging may help the diagnosis in such cases. 


\section{E1059 - STUDY OF THE APPROPRIATE DELIVERY METHOD FOR PREGNANCY WITH CARDIAC DISEASE USING NON INVASIVE CARDIAC MONITORING} Sawada M. ${ }^{1}$, Kyomoto M. ${ }^{1}$, Matsuzaka Y. ${ }^{1}$, Mizuno Y. ${ }^{1}$, Tsukinaga R. ${ }^{1}$, Konishi T. ${ }^{1}$, Shionoiri T. ${ }^{1}$, Nakanishi A. ${ }^{1}$, Horiuchi C. ${ }^{1}$, Tsuritani M. ${ }^{1}$, Kamiya C. ${ }^{1}$, Iwanaga N. ${ }^{1}$, Yoshimatsu J. ${ }^{1}$

National Cerebral and Cardiovascular Center, Perinatology, Osaka-Japan ${ }^{1}$

\section{Objective}

During labor, there are many physiological changes in circulation which may induce adverse event in pregnant woman with cardiac disease. Previously, invasive and intermittent measurement methods were used to determine the hemodynamic parameters. However, these techniques are not reliable to detect rapid hemodynamic changes during labor. The purpose of this study is to clarify how each delivery method affects cardiac function using non-invasive and continuous measurement method. That will help to indicate the appropriate delivery method for pregnancy with cardiac disease.

\section{Methods}

Prospective study was performed at National Cerebral and Cardiovascular Center in Japan from October 1, 2014 to November 30, 2018. Recruited healthy heart pregnant women were classified into 3 groups according to the delivery method; vaginal delivery without epidural anesthesia, vaginal delivery with epidural anesthesia, and caesarean section. The hemodynamic parameters; cardiac index (CI), stroke volume index (SVI) and heart rate (HR) were measured continuously during delivery by non-invasive Electrical Cardiometry monitor AESCULON mini ${ }^{\circledR}$. Interrupted time-series analysis (ITSA) was conducted to evaluate the trends and changes.

\section{Results}

Ten cases of vaginal delivery without epidural anesthesia, ten cases of vaginal delivery with epidural anesthesia, and the other ten cases of caesarean section were analyzed. In vaginal delivery, CI and HR was significantly increased before delivery. And the increase in CI and HR was mild in epidural group compared with non-epidural group. SVI was increased towards the delivery in epidural group. And it was unchanged in non-epidural group. But there was no difference in the level of outcome values between the two groups. In caesarean section, SVI was increased and HR was decreased before delivery. And after delivery, while SVI was continued to be increased and HR was not changed, CI was increased. 


\section{Conclusion}

Autotransfusion caused by uterine contraction increases venous return. Pain and anxiety according to uterine contraction stimulate the sympathetic nerve and increases HR. By using epidural anesthesia, the increase in HR before vaginal delivery was suppressed. It may reduce the cardiac load caused by tachycardia. In non-epidural vaginal delivery, the increase in venous circulation by autotransfusion is processed by increasing HR. On the other hand, in epidural vaginal delivery, it is not processed only by increasing HR but also by increasing SVI. Even though, as epidural anesthesia increases the vascular capacity, the level of SVI outcome was equivalent. In caesarean section, SVI was increased due to hydration. And HR was decreased due to the spinal anesthesia which suppress the sympathetic nerve. As a result, CI was not changed before delivery. After the delivery, as the hydration was continued, SVI was increased and CI was also increased. Spinal anesthesia reduces the heart load in caesarean section. However, hypotension and excessive infusion load must be taken care of. As there are many circulatory factors to control, it would not be the first choice for many cardiac disease patients. Summarizing for the above reasons, vaginal delivery with epidural anesthesia seems to be the best delivery mode for most cardiac disease patients. 
E1126 - THE INFLUENCE OF ACCURACY OF ESTIMATED FETAL ABDOMINAL CIRCUMFERENCE ON EMERGENCY CESAREAN SECTION IN NULLIPAROUS $\underline{\text { Kwon H. }^{1}}{ }^{1}$, Park H. ${ }^{1}$

Dongguk University Ilsan Hospital, Dongguk University College of Medicine, Obstetrics and Gynecology, Goyang-Korea, South ${ }^{1}$

\section{Objective}

This study was to investigate the risk of emergency cesarean section according to the differences between estimated fetal abdominal circumference and postnatal abdominal circumference.

\section{Methods}

A retrospective analysis from nulliparous women with singleton term births who attempted a vaginal delivery was conducted. Pregnancies with preeclampsia, chronic hypertension, diabetes, planned cesarean section, placenta previa or cesarean section due to fetal anomalies or maternal condition were excluded. Postnatal abdominal circumference was analyzed in five groups $(28-31,32,33,34$, and $35-40 \mathrm{~cm})$. Estimated fetal abdominal circumference was defined as measured data within 1 week from delivery. Odds ratio (OR) and confidence interval (CI) for emergency cesarean section according to the difference between estimated and postnatal abdominal circumference in each group were calculated after adjusting for maternal age, gestational age, and pre-pregnancy body mass index (BMI).

\section{Results}

Among the 1,129 women we analyzed, 790 (70.0\%) had a vaginal delivery and 339 (30.0\%) underwent emergency cesarean section. The estimated abdominal circumference was greater in the emergency cesarean section group compared to that in the vaginal delivery group (33.5 \pm 1.89 vs. $33.09 \pm 1.65, \mathrm{P}<0.05)$. There were no significant difference in postnatal abdominal circumference and the difference between estimated and postnatal abdominal circumference. Estimated abdominal circumference The risk of emergency cesarean section were increased with greater difference between estimated and postnatal abdominal circumference with adjusting for maternal age, gestational age at delivery and pre-pregnancy BMI. when postnatal abdominal circumference was 32cm (OR 1.10, 95\% CI 1.01-1.22) and 35cm (OR 1.26, 95\% CI 1.01-1.55).

\section{Conclusion}

The bigger difference between estimated and postnatal abdominal circumference is etiological to a considerable proportion of emergency cesarean sections in specific range. 


\section{E1194 - DINOPROSTONA VAGINAL SLOW RELEASE SYSTEM (PROPESS) FOR INDUCTION OF LABOUR A RETROSPECTIVE DESCRIPTIVE REVIEW Abian $N .{ }^{1}$, Ruiz J. ${ }^{1}$, Royo B. ${ }^{1}$, Gaston B. ${ }^{1}$, Martinez M. ${ }^{1}$, Sanz O. ${ }^{1}$, Azcona B. ${ }^{1}$,} Fernandez A. ${ }^{1}$, Echavarren V. ${ }^{1}$

Hospital Reina Sofía, Obstetrics and Gynecology, Tudela-Spain ${ }^{1}$

\section{Objective}

To analyze obstetric and perinatal outcome in single pregnancies undergoing induction of labour with Dinoprostona vaginal slow-release system (PGE2) in a secondary hospital

\section{Methods}

We performed a retrospective case series study based on 801 single gestations deliveries attended in our hospital in 2018. Pregnancies having induction of labour with PGE2 were analyzed. Obstetric and perinatal variables were reviewed.

\section{Results}

A total of 108 singletons were induced with PGE2 in 2018 (13,5\% of the total of deliveries). Mean maternal age was 32,4 years (SD 5,63). The average BMI calculated was 26.59 (SD $5,23)$. Observing obstetric background, we identify $65,7 \%$ of nulliparous pregnant patients and up to $34,3 \%$ of patients had at least one previous vaginal delivery. In this group, $40,5 \%$ of cases were multiparous (two or more previous vaginal deliveries). Previous cesarean section was also found in up to $17,6 \%$ of patients (19 patients). Most frequent medical indications were: nonreassuring fetal status (26,4\%), podalic presentation (26,4\%), failed induction (20,5\%), maternal choice (15,8\%) and cephalopelvic disproportion (10,9\%). Mean estimated fetal weight at third trimester was $3.214,07 \mathrm{~kg}$ (SD 400,92). We objectify a median for gestational age at delivery of $40+2$ weeks $(36+4-41+6)$. The duration of the induction of labour (from the time PGE2 dispositive was placed intravaginally until birth) resulted in 21,29 hours on average (SD 10,86). New born mean weight at birth was 3.289,81 $\mathrm{kg}$ (SD 495,86). The average arterial ph obtained was 7,27 (SD 0,07). Median for Apgar test was 9 (3-9) and 10 (1-10), for first and fifth minute of live, respectively. In up to $25 \%$ of cases, meconium liquid was objectified during labour. Regarding medical indications for labour induction, we observed: ruptured amniotic membranes (33,3\% of patients), prolonged pregnancy (30,6\%), oligoamnios $(17,6 \%)$, intrauterine growth restriction (6,5\%), preeclampsia $(4,6 \%)$, gestational diabetes $(4,6 \%)$ and cholestasis (2,8\%). In relation to Bishop score before initiating induction of labour with PGE2, up to 45,37\% of patients had less than 3 points, and 54,63\% obtained 3 or more points. Considering type of delivery after induction with PGE2, vaginal delivery was registered in up to $80,6 \%$ of cases (73,5\% eutocic birth, $23 \%$ vacuum extraction, forceps $3,5 \%$ ), and cesarean section occurred in up to $19,4 \%$ of cases (21 patients). Analyzing motivations for cesarean section, we found failed induction in up to $33,3 \%$ of patients (7 cases), failure to progress during labour in $28,6 \%$ of cases (6 patients), nonreassuring fetal status in $23,8 \%$ and cephalopelvic disproportion in up to $14,3 \%$. Examining neonatal outcomes, we identify 4,6\% of newborns requiring admission in Intensive Care Unit (ICU).

\section{Conclusion}

In our clinical practice, PGE2 is commonly used as first option in singleton induction of labour when no or few contractions are reveled and Bishop score is less than 5 . This mechanism may also be securely used, by performing close maternal and fetal clinical followup, in multiparous and previous cesarean section patients. 


\section{E1246 -SUCCESSFUL IVF PREGNANCY AFTER ENDOMETRTIOSIS AND THROMBOPHILIA}

Sasic M. $^{1}$

Belmedic Hospital, Gynecology and Obstetrics, Belgrade-Serbia ${ }^{1}$

Endometriosis with multi complications as the cause of sterility - Case study: Ivana F. born in 1984 from Belgrade, was treated from 2007-2016. Her first visit was in January 2007. UltraSound/2D/3D Color Doppler DG: Tumor ovarii lat dex.obs Endometriosis. Hydrosalpinx ovarii lat.dex Cystae ovarii lat sin. Endometriosis. OP: Coellioscopia. Cystectomia ovarii bill. HP Endometriosis. th ; Zoladex amp. After HS an US Color Doppler: Dg: Multiplices cystae ovarii l. dex et paraovarialis OP 21. 08. 2008. OP: Laparascopia. Cystectomia ovarii bill. Endometriosis Adhaesyolisis. HSG facta est. The HST was introduced after the operation: OC in coursu : years 2010, 2011, 2012 , 2013. In the year 2013 the patient planned pregnancy. As part of the examination for planned pregnancy complete laboratory, endocrinological and hematological tests were performed. Endocrinological testing revealed Sy Haschimoto and Hyperinsulinemia as part of PCOS. Therapy introduced: Euthirox and Metformin. Hematological testing confirmed presence of genetic mutation on F V and on PAI -1 4G/5G and confirmed Trombophillio congenitalis. Sy Leiden and mutation on gene PAI -1 4G/5G . Anticoagulation therapy was given during pregnancy. After observation of fertile days - it was decided that the patient be entered into IVF program. In 2014. patient was entered into IVF program. During hormonal stimulation, Fraxiparin 0, $4 \mathrm{ml} \mathrm{sc.} \mathrm{/24} \mathrm{h} \mathrm{was} \mathrm{introduced.} \mathrm{On}$ 24th of November Punctio folliculi et aspiratio oocita. 29th of November IVF /ET facta est . Result of beta hcg was negative. After unsuccessful ivf, the patient comes in for regular ultrasound check-up as a preparation for the next IVF. In February 2015. on ultrasound : Cystis ovarii 1 sin. Endometriosis. Hydrosalpinx ovarii 1 sin. Th: symptomatic. The Patient refused hormonal therapy. Explanation given was that pregnancy would be the best medicine for endometriosis. She continues to come for regular follicular tracking. In June 2015, she reports missed period with severe abdominal pain. Result beta hcg 150, positive, pregnancy occurred spontaneously. After seven days slight bleeding accompanied by severe pain. On ultrasound the endometrium thickness $12,2 \mathrm{~mm}$, gestational sack was not spotted. Immediately hospitalized at GA Clinic NF because of suspicion of EU. Immediately operated on; Laparascopia facta est . Gravid. ml. I + Obs EU. St. post haemorrhagiam intraabdominale causa ignore. St.post transfusio sangv.N II.Trombophillia Sy Leiden; PAI-1 4G/5G . St. post IVF am VII PCOS Sy Hashimoto. After seven days, on ultrasound checkup normal uterine pregnancy was verified. Regular check-ups followed and monthly hospitalizations. Introduced anticoagulation th : Fragmin 5000 ij. Utrogestan 200 , Progesteron depo 250 amp, folic acid. Euthirox , Ferrum . On 24. 07. 2015 Prenatal test, Double test performed: Low risk. Starting from 28th week of gestation, patient was hospitalized until the end of pregnancy. 22nd January, 2016. g. Partus SC. A healthy baby boy was born (3050g). 
E1266 - DINOPROSTONE VAGINAL SLOW RELEASE SYSTEM (PROPESS) IN PATIENTS WITH PREVIOUS CESAREAN SECTION A RETROSPECTIVE REVIEW

$\underline{\text { Abian N. }}{ }^{1}$, Ruiz J. ${ }^{1}$, Royo B. ${ }^{1}$, Gastón B. ${ }^{1}$, Rodrigo M. ${ }^{1}$, Martinez M. ${ }^{1}$, Azcona B. ${ }^{1}$, Sanz O. ${ }^{1}$, Fernandez A. ${ }^{1}$, Echavarren V. ${ }^{1}$

Hospital Reina Sofía, Gynecology and Obstetrics, Tudela-Spain ${ }^{1}$

\section{Introduction}

To analyze obstetric and perinatal outcome in patients with previous cesarean section and current single pregnancy undergoing induction of labour with Dinoprostone vaginal slowrelease system (PGE2) in a secondary hospital during 2018.

\section{Methods}

We performed a case series study based on 801 single gestations deliveries attended in our hospital in 2018. Pregnancies having induction of labour with PGE2 were analyzed. Obstetric and perinatal variables were reviewed and compared between two groups: patients with previous cesarean section (PCS) and patients with no previous cesarean section (NPCS). We used adequate statistic tests, considering a p-value less than 0.05 as statistically significant.

\section{Results}

A total of 108 singletons were induced with PGE2 in 2018 (13,5\% of the total of deliveries). We identify previous cesarean section in this sample in up to $17,6 \%$ of cases (19 patients). We found $65,7 \%$ of nulliparous pregnant patients and up to $34,3 \%$ of patients had at least one previous vaginal delivery. In this group, 40,5\% of cases were multiparous (two or more previous vaginal deliveries). Mean gestational age at induction of labour had no statistically significant differences between PCS and NPCS groups (p: 0,070), and neither did mean maternal age (p: 0,120) and BMI (p: 0,058). Regarding medical indications for labour induction, we observed no statistically significant differences in both groups PCS and NPCS for any of them (in order of frequency): ruptured amniotic membranes (p: 0,151), prolonged pregnancy (p: 0,164), oligoamnios (p: 0,087), intrauterine growth restriction (p: 0,169) and gestational diabetes (p: 0,220).

Considering type of delivery, normal vaginal delivery is identified as statistically significant more frequent in NPCS group as compared to PCS group (p: 0,038). In relation to instrumental delivery using vacuum and forceps, no statistically significant differences were found (p: 0,100 and 0,098, respectively). We observed no differences in the incidence of cesarean section comparing this two groups, PCS and NPCS (p: 0,012). When analyzing neonatal variables comparing PCS and NPCS groups, we detect no statistically significant differences with reference to frequency of meconium amniotic fluid (p: 0,091), arterial ph at birth $(0,116)$ and Apgar test for first and fifth minute of live (p: 0,214 and 0,134, respectively). Up to 10,53\% of newborns from PCS mothers required admission in Neonatal Intensive Care Unit (NICU), in opposition to 3,37\% of newborns from NPCS mothers; this result represents statistically significant differences (p 0,012).

\section{Conclusion}

In the light of this results, it is important to underline that no increased incidence of cesarean section was objectified in PCS group, opposed to NPCS group, after induction with PGE2. On the other hand, normal vaginal delivery appears to be statistically significant more frequent in NPCS, with no differences comparing with PCS in terms of instrumental deliveries. We found significantly more cases of admission in NICU in PCS (3 newborns). In our clinical practice, we use PGE2 in single pregnancies induction of labour even in PCS cases. This mechanism may also be securely used in this group, performing close maternal and fetal clinical follow-up 
E1343 - PLACENTAL ABRUPTION AFTER EXTERNAL CEPHALIC VERSION Pedroarena I. ${ }^{1}$, Pérez B. ${ }^{1}$, García S. ${ }^{1}$, Urtasun M. ${ }^{1}$, Abián N. ${ }^{2}$, Gastón B. ${ }^{3}$, Ruiz M. ${ }^{3}$, Bazán M. ${ }^{3}$, Barrenetxea J. ${ }^{3}$, Zabaleta I. ${ }^{3}$

Complejo Hospitalario De Navarra, Department of Obstetrics and Gynecology, Pamplona Spain $^{1}$

Hospital Reina Sofía, Department of Obstetrics and Gynecology, Pamplona-Spain ${ }^{2}$

Omplejo Hospitalario De Navarra, Department of Obstetrics and Gynecology, Pamplona Spain $^{3}$

The external cephalic version (ECV) is a set of manoeuvres that seek to convert fetal presentation into cephalic in fetus with different presentation by external manipulation through the maternal abdomen.

In a large percentage of foetuses the breech presentation is equivalent to the performance of a planned caesarean section so ECV increases the chances of vaginal delivery. Therefore it is an effective measure to decreasing the caesarean rate.

The ECV is indicated in all pregnant women who present a fetus in different presentation of the cephalic from the 37th week of pregnancy and do not present any contraindication.

\section{Case}

A 38-year-old woman with no medical or surgical history

It was about his first pregnancy. The pregnancy was well tolerated with normal evolution.

In the 38th week of pregnancy, she went to the hospital to perform an external cephalic version due to breech presentation of the fetus.

She was admitted to the delivery room for tocolysis with ritodrine and cardiotocography. Subsequently, ECV manoeuvres were performed in the operating room with ultrasound control. Finally, the external cephalic version was not achieved.

Prior to hospital discharge, a cardiotocography recording was performed for two hours. The record was compatible with fetal well-being.

Suddenly the patient started to bleed vaginally. An ultrasound was performed objectifying a chorioamniotic detachment. The fetal heart rate was normal but on suspicion of a placental abruption an urgent caesarean section was indicated.

During the caesarean, bloody amniotic fluid was seen. With the removal of the placenta, the exit of a large blood clot was also observed.

The newborn cried in the surgical field. The APGAR test at minute of life was 8. The determination of fetal artery and vein $\mathrm{pH}$ was 6.95 and 7.02 respectively. He did not need neonatal resuscitation.

The ECV is a safe procedure for the mother and the fetus. Most of the complications that occur are mild like self-limiting vaginal bleeding or transient alteration of the cardiotocographic record. However, in some cases there may be complications that can suppose a risk of loss fetal well-being. In these cases it is necessary to perform an emergency caesarean section. The risk of fetal mortality is very low. 
E1364 - A SINGLE CENTER STUDY ON RIGHT SIDED CONGENITAL DIAPHRAGMATIC HERNIAS IN NEONATES

Jeong J. ${ }^{1}$, Lee M. ${ }^{2}$, Won H. ${ }^{2}$, Namgoong J. ${ }^{3}$, Kim S. ${ }^{3}$, Kim D. ${ }^{3}$, Jung E. ${ }^{4}$, Kim E. ${ }^{4}$, Kim K. ${ }^{4}$, Lee B. ${ }^{4}$

University of Ulsan College of Medicine, Asan Medical Center, Pediatrics, Seoul-Korea, South ${ }^{1}$

University of Ulsan College of Medicine, Asan Medical Center, Obstetrics and Gynecology, Seoul-Korea, South ${ }^{2}$

University of Ulsan College of Medicine, Asan Medical Center, Pediatric Surgery, SeoulKorea, South ${ }^{3}$

University of Ulsan College of Medicine, Asan Medical Center, Neonatology, Seoul-Korea, South $^{4}$

\section{Objective}

Right-sided congenital diaphragmatic hernia (R-CDH) has not been studied extensively due to its rarity. It remains unclear that the prenatal risk stratification based on the data from left $\mathrm{CDH}$ (L-CDH) could be applied to R-CDH. The purpose of this study was to share our experience in the treatment outcome of $\mathrm{R}-\mathrm{CDH}$, focusing on the prenatal and postnatal prognostic factors.

\section{Methods}

We reviewed the medical records of newborn patients with prenatally diagnosed R-CDH managed at a tertiary center between 2006 and 2019. Subjects were classified into survivors $(n=23)$ and nonsurvivors $(n=15)$. Demographic and clinical characteristics such as size and grades of defect, liver herniation, and prenatal ultrasound-measured lung volume by observed versus expected lung area to head circumference (O/E LHR), which are known as poor prognostic factors in L-CDH, were compared and analyzed.

\section{Results}

Of 38 patients, there were 25 males and 13 females with a mean gestational age (GA) of $37 \pm$ 3 weeks, and mean birth weight of $2954 \pm 604$ g. Of the 38 cases included, 23 (60.5\%) survived to discharge. Mortality occurred before $(n=8)$ and after $(n=7)$ surgical repair. Among a total of $30(78.9 \%)$ patients who underwent surgical repair, 15 patients $(50.0 \%)$ required patch repair. Compared with nonsurvivors, survivors had significantly higher 1 minute' and 5 minutes' Apgar scores. As expected, the rate of use of high frequency ventilator $(100.0 \% \mathrm{vs}$. $52.2 \%, \mathrm{p}=0.002)$, surfactant use $(60.0 \%$ vs. $8.7 \%, \mathrm{p}=0.001)$, inhaled nitric oxide and extracorporeal membrane oxygenation is greater in the nonsurvivors versus survivors. However, GA and birth weight did not differ between the two groups. The incidence of high grades of diaphragmatic defect was associated with the mortality (OR 10.00, 95\% CI 1.39271.86, $\mathrm{p}=0.023$ ).

Among the 17 patients in whom O/E LHR was available, the O/E LHR did not differ between the survivors $(52.9 \%)$ and nonsurvivors $(47.1 \%)(p=0.167)$. The value of $\mathrm{O} / \mathrm{E} \mathrm{LHR} \geq 45$ failed to predict survival to discharge in R-CDH.

\section{Conclusions}

Our study suggests that risk stratification of R-CDH should be based on the early clinical features and surgical findings. Further research efforts are required to identify a novel prenatal marker that is reflective of lung hypoplasia of R-CDH. 


\section{Obstetrics - Multiple pregnancy}

\section{E1046 - MULTIPLE GESTATION IN UTERUS BICORNO WITH SATISFACTORY EVOLUTION OF ONE OF THE GESTATIONS AND ABORTION RETAINED IN CONTRALATERAL GESTATION IN A MATERNITY HOSPITAL IN THE AMAZON REGION CASE REPORT}

$\underline{\text { Ramires B. }}{ }^{1}$, Simões M. ${ }^{1}$

Maternidade Municipal Mãe Esperança, Residência Médica, Porto Velho-Brazil ${ }^{1}$

Mullerian malformations represent a set of structural abnormalities that may arise from the fusion of these ducts in the midline on failure to connect to the urogenital sinus, or failure to create an appropriate light in the upper portion of the vagina and uterus, by non-reabsorption of cells of the central vagina and the septum between the merged Müllerian ducts. The definitive cause of these anomalies is not clearly elucidated. Gestation in these cases presents a high rate of spontaneous abortion and even infertility.

\section{Objective}

To report a case of multiple gestations in bicornic uterus, which evolved with term gestation and spontaneous abortion, and to carry out a review in the literature on the subject?

\section{Methods}

It is a clinical case study with a basic descriptive approach with literature review. The study was carried out with a single sample, describing multiple and spontaneous gestation associated with the bicornic uterus in which there was spontaneous gestation with good obstetric evolution.

\section{Results}

SROF, 38 years old, 6s2d, G6 PC2PN1 A2, seeks maternity care with ultrasound showing multiple gestations in uterus with bicorno morphology, presenting in the right horn live embryo in gestational sac with gestation of $6 \mathrm{~s} 2 \mathrm{~d}$ and in the contralateral horn evidencing hypotonic, irregular gestational sac, with febris in its interior and bcf absent. Orientation of the pregnant woman regarding the prognosis of the current gestation and the possibility of expectant behavior on the pregnancy of retained abortion. In this way, after 26 days, the pregnant woman evolves with increased vaginal bleeding and observed the output of the gestational sac. Afterwards, a new transvaginal ultrasonography was performed, evidencing gestation in a good evolution of $12 \mathrm{~s} 2 \mathrm{~d}$ and contralateral bicornar uterus, without ovular remains. A complete abortion is at the moment complete. During gestational period, the mother had no episodes of gestational complications as well as good fetal development. Elective cesarean section performed at 40 weeks in the maternity of the Amazon region, with exit of live fetus, apgar 8/9, with 3,215 g. Performed neonatal care and cesarean section procedure according to technique, as well as puerperal care.

\section{Conclusion}

In this case, we describe multiple gestation in a bicornic uterus in which there was spontaneous gestation with good obstetric evolution. From a reproductive point of view, this Mullerian malformation is associated with abortion and infertility. In the clinical case, it revealed a multiparous patient, with no surgical treatment for the abortion, patient presenting himself. 
In our case, the patient was asymptomatic, both from the reproductive and clinical points of view, and had spontaneous gestation without intercurrences, with a healthy, full term newborn. These findings could reveal a gestation with preterm labor and other obstetric complications that could be attributed to this anatomical anomaly, but here there was a good clinical, obstetric and neonatal outcome. 


\section{E1079 - A CASE WITH OVARIAN HETEROTOPIC PREGNANCY TO EXPECT THE UNEXPECTED}

Zafer E. ${ }^{1}$, Çetin Yılmazlar N. ${ }^{1}$, Atakul T. ${ }^{1}$, Kuh B. ${ }^{1}$, Ertekin A. ${ }^{1}$

Aydin Adnan Menderes University, Obstetrics and Gynecology, Aydin-Turkey ${ }^{1}$

Heterotopic pregnancy, the presence of intrauterine and extrauterine (ectopic) gestation simultaneously, occurs in about $1 / 30000$ pregnancies. Its incidence has increased in recent decades secondary to a relative increase in artificial reproductive procedures. In busy obstetric clinics, it is hard to keep in mind the rare abnormalities. However, heterotopic pregnancy stays as a potential morbid condition if not diagnosed early. Here we present a heterotopic pregnancy case, a 26 years old Gravida 4, Parity 1 case who was at 6w2d of gestation by her last menstrual period was presented with mild pelvic pain and vaginal bleeding. Her vital signs were normal. The patient had mild abdominal tenderness to palpation. She had no active vaginal bleeding. Transvaginal ultrasound showed an intrauterine embryo with crown-rump length (CRL) of 6w4d along with cardiac activity. A moderate amount of free fluid (blood) was also observed. A more careful look revealed a 9.5mm hypoechoic cyst in the left adnexal region consistent with a gestational sac having $2.5 \mathrm{~mm}$ hyperechoic structure inside, which was suspected to be embryo. Complete blood count showed mild anemia (hemoglobin 10 $\mathrm{g} / \mathrm{dL}$ ). Serum $\beta$-hCG level was $7351 \mathrm{mIU} / \mathrm{mL}$. Heterotopic pregnancy diagnosis was presumed. Management options were discussed with the patient. The patient was taken to the operating room and diagnostic laparoscopy was performed. A left ovarian ectopic pregnancy was seen with approximately $200 \mathrm{~mL}$ intraabdominal bleeding. Left ovarian ectopic pregnancy removal was achieved while the ovary was left intact. After the procedure, the patient was then taken to the regular obstetric post-operative floor. She was then discharged from the hospital in stable condition. The result of her pathology reported was consistent with ovarian ectopic pregnancy. The patient was followed up until the 27th gestational week, when she had still an uneventful pregnancy so far, at the time of this presentation. Her medical history was negative for assisted reproductive procedures (ART), pelvic surgeries or endometritis/salpingitis. Some previous reports have described higher spontaneous miscarriage rates in heterotopic pregnancies than intrauterine-only pregnancies (up to $30 \%$ ). Our case did not have a first-trimester miscarriage. Ovarian implantation is uncommon even for singleton ectopic pregnancies; it's very rare in heterotopic pregnancies. The presented case had heterotopic ovarian pregnancy. Local methotrexate injections for the management of heterotopic pregnancies have been reported however, in bleeding heterotopic pregnancies with desired live intrauterine embryos, a surgical option may be the most appropriate approach. Here we presented an ovarian heterotopic tubal pregnancy managed with laparoscopic removal on whose pregnancy continued until 30th weeks so far, by the time this presentation was being prepared. Her negative history for previous tubal manipulation, infection or ART was unusual. Therefore, the first ultrasound examination of seemingly lowrisk pregnancies should always include purposeful and careful look for ruling out multiple and/or heterotopic pregnancies rather than measuring CRL and confirming fetal cardiac activity alone. 
E1103 - BIRTH WEIGHT REFERENCE PERCENTILES BY GESTATIONAL AGE FOR TURKISH TWINS

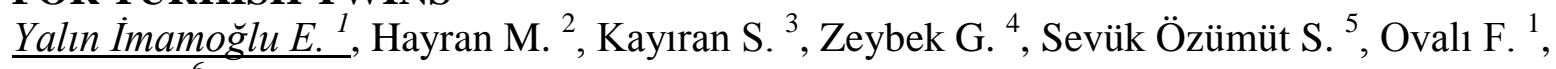
Gürsoy T.

Istanbul Medeniyet University Göztepe Training and Research Hospital, Neonatology, Istanbul-Turkey ${ }^{1}$

Hacettepe University Faculty of Medicine, Preventive Oncology, Istanbul Turkey ${ }^{2}$

American Hospital, Pediatrics, Istanbul-Turkey ${ }^{3}$

Faculty of Health Sciences Umraniye Training and Research Hospital, Pediatrics, IstanbulTurkey ${ }^{4}$

Istanbul Medeniyet University Göztepe Training and Research Hospital, Pediatrics, IstanbulTurkey ${ }^{5}$

Koç University School of Medicine, Neonatology, Istanbul-Turkey ${ }^{6}$

\section{Objective}

Birth weight of twins are known to be less than those of comparable singletons and twinspecific birth weight percentile curves are recommended for use in clinical practice. Several countries have developed nation-specific birth weight references for twins, however Turkey still lacks such references. The aim of this study was to develop birth weight reference percentiles by gestational age for Turkish twins.

\section{Methods}

The birth records of all consecutive pregnancies resulting in twin births between 2009 and 2018 were reviewed. Only Turkish live twin births without birth defects between 24 and 42 weeks of gestation were included.

\section{Results}

A total of 2546 twin neonates were included in the analysis and smoothed birth weight percentile curves by gestational age were constructed.

\section{Conclusion}

The established birth weight percentiles represent the first birth weight nomogram for contemporary Turkish twins and could be a useful tool to assess growth of twins in clinical and research settings. 
E1149 - THORACO OMPHALOPAGUS TWINS A SIMULATOR THAT HELPED TO PERFORM THIS DIFFICULT FETAL EXTRACTION AT CESAREAN DELIVERY Pivetta Cantarelli M. ${ }^{1}$, Souza Da Correggio K. ${ }^{1}$, Knobel R. ${ }^{1}$, Salermo Wilkens R. ${ }^{1}$, Franco M. ${ }^{1}$, Simas Abi Saab J. ${ }^{1}$, Galuzzo Noya R. ${ }^{1}$, Koettker Silveira S. ${ }^{1}$, May Feuerschuette O. ${ }^{1}$, Nascimento Pacheco E. ${ }^{1}$, Rossi dos Santos G. ${ }^{1}$, Ronconi Tomaz R. ${ }^{1}$, Trapani Jr. A. ${ }^{1}$ Hu/ufsc/ebserh/ Women's Health Care Unit, Gynecology and Obstetrics, Florianópolis-Brazil ${ }^{1}$

Conjoined twins are rare, and in the literature their incidence ranges from 1:50,000 to 1:100,000 live births. This anomaly occurs when exist the joining of two identical twins who share one or more organs. As the Spencer classification, Thoracopagus twins are united face to face from the upper thorax to the umbilicus with a common sternum, diaphragm, and upper abdominal wall. Meanwhile, Omphalopagus twins are joined ventrally in the abdomen, often including the lower thorax.

This paper objective is to report a rare case of conjoined twins and to show how the fetal extraction, in the cesarean section, was planned using a simulator.

According to the Spencer classification, the case twins could be classificated as Thoracoomphalopagus. In the other words, they were joined at the chest and at the abdomen and had one functional heart (IMAGE 1). Because the bad fetus prognosis, the obstetricians debated the better method to decrease surgery risks. When the twins were 35 weeks, the patient was submitted to a cesarean section. Before the surgery, a simulator was made with a pelvis model (Simulaids ${ }^{\circledR}$ ) and two dolls bonded with tape. It was used to simulate the twins position seen previously by ultrasound. The objective was to minimize que uterus damage, to reduce maternal morbidity to the possible future pregnancies, who had just one child and aim to have more. During the procedure, the surgeons repeated the simulated movements they had just trained with the simulator model, and they could successfully perform the difficult fetal extraction with just a normal transversal uterus incision and with decreased surgical time. 
E1168 - NATURAL HISTORY OF MONOCHORIONIC DIAMNIOTIC TWIN PREGNANCIES WITH AND WITHOUT TWIN TWIN TRANSFUSION SYNDROME Ribeiro Ramalho A. ${ }^{1}$, Fernandes Freitas Maia J. ${ }^{2}$, Caminha Ramires B. ${ }^{3}$, Ribeiro De Oliveira E. ${ }^{2}$, Ribeiro Simoes M. ${ }^{3}$

Maternidade Municipal Mae Esperança, Gynecology and Obstetrics, Porto Velho-Brazil ${ }^{1}$ Hospital De Base Ary Pinheiro, Gynecology and Obstetrics, Porto Velho-Brazil ${ }^{2}$

Maternidade Municipal Mae Esperanca, Gynecology and Obstetrics, Porto Velho-Brazil ${ }^{3}$

\section{Objective}

To evaluate the evolution of monochorionic-diamniotic twin pregnancies with and without the twin-twin transfusion syndrome (TTTS), followed up in an expectant way. Methods: retrospective study in which the pregnancies with and without TTTS and with mild (Quintero's stage I) and severe (Quintero's stages II, III, IV and V) disease manifestations were compared according to extreme preterm delivery, neurological impairment and the twins' nursery discharge. The extreme preterm twins who had had TTTS, or not, were compared whether they had or not neurological impairment. The $\chi 2$ or Fisher's exact test were used.

\section{Results}

Among 149 monochorionic-diamniotic twin pregnancies, 15 presented TTTS, 11 (11/15 $73.3 \%)$ in the severe form and $4(4 / 15-26.7 \%)$ at stage I. The extreme preterm delivery was more frequent $(\mathrm{p}<0.001)$ in the cases with the disease $(11 / 15$ - 73.3\%) than in the cases without it $(25 / 134-18.7 \%)$, and more common $(\mathrm{p}=0.033)$ in severe $(10 / 11-91.1 \%)$ than in mild cases (1/4 - 25.0\%). Neurological impairment in at least one twin was more frequent in cases with $(5 / 8-62.5 \%)$ than in cases without $(9 / 134-6.7 \%)$ the disease $(p<0.001)$. Nursery discharge of at least one twin was more common $(p<0.001)$ in cases without $(132 / 134$ $98.5 \%)$ than in cases with the disease (8/15 - 53.0\%). Neurological impairment in at least one of the twins was more frequent $(\mathrm{p}=0.04)$ in the severe $(5 / 5-100 \%)$ than in the mild $(1 / 4$ $25 \%)$ form of the disease. Nursery discharge of both twins was more common $(p=0.004)$ at stage I (4/4 - 100\%), than in the severe form of the disease $(1 / 11-9.0 \%)$. Among the 47 extreme preterm twins, the neurological impairment was more frequent $(\mathrm{p}=0.001)$ among the ones who had (6/6 - 100\%), than among those who did not have TTTS (11/41 - 26.8\%).

\section{Conclusion}

Cases with twin-twin transfusion syndrome, followed up in an expectant way have bad perinatal prognosis, with high neonatal mortality and high rates of neurological arrest among the survivors. 


\section{E1236 - DELAYED INTERVAL DELIVERY IN TWIN PREGNANCY REPORT OF CLINICAL CASES \\ Guerra M. ${ }^{1}$, Galleguillos C. ${ }^{1}$, Haye M. ${ }^{1}$, Silva M. ${ }^{2}$, Gutierrez J. ${ }^{1}$}

Hospital Clinico San Jose, Ginecoobstetricia, Santiago-Chile ${ }^{1}$ Hospital Clinico Universidad

De Chile, Ginecoobstetricia, Santiago-Chile ${ }^{2}$

\section{Introduction}

The twin pregnancy is associated with a higher probability of premature birth, $16 \%$ and $4 \%$ will have a delivery respective before 34 and 30 weeks, with a high risk of morbidity and mortality. In those pregnancies that initiate extreme premature labor, it is possible to try to prolong the gestation of the second twin, improving the survival and decreasing morbidity. This situation is defined as delayed delivery or asynchronous delivery of the second twin, first described by Carson in 1980. Our objective is to disclose four cases of delayed-interval delivery in twin pregnancy to show that when facing the birth of an extremely premature twin is possible to delay the birth of the second or third fetus, using active management with cerclage and antibiotics, improving the neonatal prognosis.

\section{Methods}

A retrospective study was carried out analyzing the cases of extreme premature delivery of the first twin and deferred delivery of the second, between 2014 and 2017, at the Clinical Hospital of the University of Chile, Hospital San José and Clínica Indisa. The variables analyzed were: chorionicity, number of fetuses, maternal age, type of conception, gestational age at the time of delivery of the first twin, latency at delivery of the second twin, and management strategies.

\section{Results}

There were 4 cases of extreme premature labor and delayed delivery, 3 of them in double twin pregnancies and one triple, there were no cases in monochorionics. Mean maternal age was 33 years, and half of the cases were patients with in vitro fertilization. The mean gestational age at first delivery was $18+5$ weeks, and the birth interval was 23 to 153 days (median 83.5 days).

The management strategy was McDonald cerclage within the first hour of delivery of the first fetus once the cord was ligated and placenta in situ and broad spectrum antibiotics. The birth of the second / third fetus was in $100 \%$ of the cases by caesarean section whereas the first one was vaginal. The overall survival rate was $80 \%$, being $100 \%$ in the cases of double pregnancies. In the case of triple pregnancy, one of the newborns die on the 27th day. $100 \%$ of the newborns that survive are neurologically healthy and no maternal morbidity associated with the management was recorded.

\section{Conclusion}

In multiple pregnancies with extremely premature birth of a twin, delaying the birth of the second is a valid and safe option to improve the neonatal prognosis and is not associated with higher maternal morbidity. 
E1296 - RESULTS OF EMERGENCY CERVICAL CERCLAGE AND TOTAL CERVIX OCCLUSION IN TWIN PREGNANCIES Tsikouras P. ${ }^{1}$, Deuteraiou D. ${ }^{1}$, Anthoulaki X. ${ }^{1}$, Bothou A. ${ }^{2}$, Chalkidou A. ${ }^{1}$, Gaitatzi F. ${ }^{1}$, Babageorgaka E. ${ }^{1}$, Tsirkas I. ${ }^{1}$, Michalopoulos S. ${ }^{1}$, Koukoulomati A. ${ }^{1}$, Soilemtzidis M. ${ }^{1}$, Zervoudis S. ${ }^{3}$, Galazios G. ${ }^{1}$, Csorba R. ${ }^{4}$, Teichmann A. ${ }^{4}$

Democritus University of Thrace, Obstetrics and Gynecology, Alexandroupolis-Greece ${ }^{1}$

Democritus University of Thrce, Obstetrics and Gynecology, Alexandroupoolis-Greece ${ }^{2}$

Rhea Hospital, Obstetrics and Gynecology, Athens-Greece ${ }^{3}$ Teaching Hospital

Aschaffenburg, Obstetrics and Gynecology, Aschaffenburg-Germany ${ }^{4}$

\section{Objective}

Emergency cerclage was performed when the bulging fetal membranes reached or overcame the external os and when total cervical dilation was present. In this retrospective study apart from the results were evaluated as well as the effects of emergency cerclage in combination with total cervix occlusion.

\section{Methods}

In order to evaluate the current management of pregnancies in risk for abortion or very preterm birth a retrospective analysis was conducted in Clinicum Aschaffenburg, Teaching Hospital of University Würzburg of Germany, between January 1995 and July 2016. To all the study patients, 105 in total, with absence of infection or labor and uncomplicated pregnancy, was performed an emergency cerclage according to Shirodkar technique and total cervical occlusion as described by Szendi.

\section{Results}

The mean age of the pregnant study women was $29.3 \pm 0.5$ years old and the mean gestational age at the time of the emergency cerclage was 14.9 weeks (range 12-18). The risk factors in these pregnancies are as following: mean maternal age $>35$ years: 37(35.23\%), smoking: 45 (42.85\%), history of cone biopsy: 4 (3.8\%), history of Mullerian anomaly: 2(1.8\%), previous miscarriages:24 (22.85\%).101 out of 105 participants delivered after the 27th week of gestation (96.1\%) with two study women having an abortion at the 22th week of gestation four days after the completion of the procedure and the other two women aborted at the 23th week ten days after the performance of cerclage. From the 101 pregnant women 5 (4.7 \%) (delivered at term (greater than 37 week) and 85 (80.9\%) between the 32nd and 34th week of gestation whereas 11(10.4\%) between 29 and 31 pregnancy week. For the improvement of cerclage efficiency, in follow up treatments were included bed rest, intravenous tocolysis, antibiotics, progesterone per os administration simultaneously intramuscular injection per week and in some cases the Trendelenburg position.

\section{Conclusion}

Emergency cerclage may prolong the twin pregnancy but it results in a high rate of preterm deliveries. 
E1310 - MONOAMNIOTIC MONOCYTIC GEMELLAR PREGNANCY IN PREGNANT WOMEN ATTENDING AN OUTPATIENT CLINIC IN THE WESTERN AMAZON CASE REPORT

Ribeiro Simões M. ${ }^{1}$, Gloria Da Silveira E. ${ }^{2}$, Simões Lamego L. ${ }^{3}$, Simões Lamego G. ${ }^{4}$, Ribeiro Freitas P. ${ }^{5}$, Pandolfi Freitas T. ${ }^{1}$

Faculdades Integradas Aparicio De Carvalho, Medicina, Porto Velho-ro-Brazil ${ }^{1}$

Universidade Federal De Rondônia, Geografia, Porto Velho-ro-Brazil ${ }^{2}$

Hospital Getúlio Vargas, Urgência E Emergência, Tramandai-rs-Brazil ${ }^{3}$

Gso Medicina Ocupacional, Medicina Do Trabalho, Esteio-rs-Brazil ${ }^{4}$

Clinica Maria Do Carmo, Ultrassonografia, Porto Velho-ro-Brazil ${ }^{5}$

\section{Introduction}

Gemelarity occurs in approximately $1 \%$ of all pregnancies and is linked to increased perinatal morbidity and mortality when compared to single gestation. Monocorionicity has been shown to be the most important isolated risk factor in twin pregnancies, this risk increases when monoamniotic, monoamyotic monocorionic twin pregnancies are associated with a higher risk of fetal malformation, prematurity, fetus-fetal transfusion syndrome and Intra-utero fetal death, by the folding of umbilical cords and interruption of flow in these. The diagnosis of Corionicity is performed by early ultrasonography in the first trimester.

\section{Objective}

To report a case of monochorionic monoamniotic gestation and its possible complications, as well as to review literature of greater relevance on the theme

\section{Methods}

The present work uses the reference of bibliographic research in order to search for information and report the rare case of a monoamniotic monocorionic twin pregnancy and its complications in a pregnant woman attended at an outpatient clinic in the Western Amazon.

\section{Case}

LLS,25 years old, twin gestation, monoamniotic monocorionics, 26s2d, G3PN1A1, attended the consultation in high-risk prenatal care on day 11/12/18, presenting chronic arterial hypertension, in use of Methyldopa 750mg a day and AAS $100 \mathrm{mg} /$ day. Presented VDRL 1/2 and made Treatment with Bezetacil 2.4 million weekly in three doses. On day 28/02/19 with 37S4D he presented colic pain and was referred to the referral hospital for high-risk pregnancy for evaluation. The physical examination showed: PA:150x100mmHg, uterine height: 40cm; uterine dynamics: absent; fetal movements: both present; fetal heartbeat: fetus1:140 bpm and fetus 2:150 BPM. Hospitalization was performed and cesarean delivery was carried out, with weight fetus1:2400g and fetus2:2550g APGAR fetus1:8/9 and fetus2:8/9 not occurring intercurrences both newborns and mother.

\section{Conclusion}

Prenatal follow-up of multiple pregnancies should be oriented to enable early diagnosis of the alterations, being the first trimester ultrasound important for the determination of placentation, and the establishment of the prognosis and Conduct of these pregnancies. As well as perform routine exams and treat any complications. 


\section{E1331 - AUDIT ON ANTENATAL MANAGEMENT OF MULTIPLE PREGNANCIES IN A DISTRICT GENERAL HOSPITAL IN THE UK}

Garaeva I. ${ }^{1}$, Poozhikalayil S. ${ }^{1}$

Great Western Hospital, Obstetrics and Gynaecology, Swindon-United Kingdom ${ }^{1}$

\section{Introduction}

Multiple pregnancy accounts for approximately 3\% of live births in the UK with a significant rise in incidence of multiple births over the last 30 years. Multiple pregnancy is associated with higher risk of adverse outcomes for both mothers and babies. In view of the recognised increased risks of complications, NICE introduced a multiple pregnancy guideline in 2011 with care recommendations to guide the antenatal assessment and management of multiple pregnancies.

\section{Objective}

1) To assess the antenatal management of multiple pregnancies in Great Western Hospital using specific criteria from NICE guidelines as standards of care.

2) To assess maternal and foetal complications in multiple pregnancies delivered at GWH.

\section{Methods}

The patient sample comprised all women with multiple pregnancies who booked at GWH and had most or all of their antenatal care at GWH over a twelve-month period from April 2017 to March 2018. Data was obtained retrospectively by reviewing maternal health records as well as hospital electronic records.

\section{Results}

Seventy patients were identified and the prevalence of MCDA, DCDA and triplet pregnancies was $17.1 \%, 81.4 \%$, and $1.4 \%$, respectively. There was an $88 \%$ uptake of Down's Syndrome screening. NICE recommends different scan frequencies for multiple pregnancies and the adherence was over 98\% for both MCDA and DCDA pregnancies. Elective delivery at recommended gestations was offered in $100 \%$ of uncomplicated multiple pregnancies. NICE advises a targeted use of antenatal corticosteroids which was achieved in only $62 \%$ of patients.

The prevalence of raised BMI $(\geq 25)$ in this patient sample was $53 \%$. Maternal complications included preterm labour, hypertensive disorders, postpartum haemorrhage and gestational diabetes, and were $23 \%$, 7\%, 54\% and 7\% respectively. Overall, $41 \%$ of patients delivered by vaginal delivery, $32 \%$ delivered by elective caesarean section and the remaining $27 \%$ required an emergency caesarean section.

The prevalence of intrauterine growth restriction in this population was $19 \%$. There was one case of stillbirth in MCDA pregnancy following a severe IUGR early in pregnancy, with the second twin suffering a neonatal death shortly after delivery.

\section{Conclusion}

This audit demonstrated compliance with majority of NICE recommendations in the antenatal management of multiple pregnancies. This audit showed that prophylactic (untargeted) course of steroids was given in 38\% of multiple pregnancies, and hence represents an area for improvement of care in the future. 


\section{E1333 - HYPERREACTIO LUTEINALIS IN TWIN PREGNANCY WITH UNEXPECTED ADVERSE PREGNANCY OUTCOME}

Skrgatic L. ${ }^{1}$, Radosevic V. ${ }^{2}$, Vecek N. ${ }^{2}$, Strelec M. ${ }^{3}$, Kralik Oguic S. ${ }^{4}$, Gelo N. ${ }^{5}$

University of Zagreb School of Medicine, Division of Gynecological Endocrinology and

Reproductive Medicine, Clinical Hospital Centre Zagreb, Zagreb-Croatia ${ }^{1}$

Clinical Hospital Centre Zagreb, Division of Perinatology, Zagreb-Croatia ${ }^{2}$ Clinical Hospital Centre Zagreb, Division of Gynecological Endocrinology and Reproductive Medicine, Zagreb-Croatia $^{3}$

Clinical Hospital Centre Zagreb, Division of Laboratory Medicine, Zagreb-Croatia ${ }^{4}$

Clinical Hospital Centre Zagreb, Division of Embryology, Zagreb-Croatia ${ }^{5}$

Case report describes pregnancy management and outcome of bichorionic biamniotic (BCBA) twin pregnancy with Hyperreactio luteinalis (HL) and accompanying severe hyperandrogenemia in women carrying female fetuses. HL with hyperandrogenism is rare condition, which may cause virilization of the mother and female fetus. According to limited data from current literature adverse pregnancy outcomes in these women are observed in $26 \%$ of cases. They are usually associated with preeclampsia, gestational diabetes and preterm delivery. The risk to the fetus depends on the timing and severity of the excess maternal androgen production.

35-year-old primigravida with twin pregnancy following frozen embryo transfer in natural cycle presented in 16th week of pregnancy with bilateral enlarged ovarian masses

The hormone panel showed extremely elevated serum total testosterone (TT) $27.3 \mathrm{nmol} / \mathrm{L}$, free testosterone (FT) $62.2 \mathrm{pmol} / \mathrm{L}$, androstenedione (A) > $35 \mathrm{nmol} / \mathrm{L}$ levels. MRI of abdomen and pelvis ruled out androgen secreting tumor. The HL was diagnosed. Feto-placental unit and hormones were monitored every 3 weeks. Hyperandrogenemia was progressive and in 33rd week discordant twin growth was observed accompanied with hypertension. Respiratory distress syndrome prophylaxis was preformed and methyldopa was introduced. In 34th week absent umbilical artery end-diastolic flow was detected in one twin. Within 24 hours affected twin heartbeats were negative after two reassuring CTGs. Cesarean section was immediately performed. No signs of virilization of twins were observed. Umbilical cord serum androgen concentrations have been measured in twins. Stillborn twin had significantly higher androgen levels compared to liveborn twin (TT 5.5 vs. $2.2 \mathrm{nmol} / \mathrm{L}$, fT 131.5 vs. 51.0 pmol/L, A 33.1 vs. $8.5 \mathrm{nmol} / \mathrm{L}$ ). Pathological report was unremarkable. The mother's androgen panel on the third postpartal day was still extreme (TT $39.4 \mathrm{nmol} / \mathrm{L}$, fT $92.5 \mathrm{pmol} / \mathrm{L}$, A $250 \mathrm{nmol} / \mathrm{L}$ ). A control hormonal panel two weeks later normalized. Hyperandrogenism in pregnancy complicated with fetal brain-sparing effect could lead to accelerated exhaustion of fetal compensatory mechanisms. Therefore, in such situation prompt delivery may be a reasonable option. 


\section{E1380 - HETEROTOPIC - BILATERAL TUBAL ECTOPIC PREGNANCY AFTER TRANSFER OF 3 EMBRYOS A DIAGNOSIS NOT TO MISS}

Velegrakis A. $^{1}$, Matalliotakis M. ${ }^{1}$, Demosthenous E. ${ }^{1}$, Sifakis S. ${ }^{2}$

Venizeleio General Hospital, Department of Obstetrics and Gynecology, Heraklion-Greece ${ }^{1}$

Mitera Maternity Hospital, Heraklion- ${ }^{2}$

\section{Case}

Simultaneous occurrence of intrauterine and ectopic pregnancy, defined as heterotopic pregnancy, is rare; however its incidence increases with the use of the several methods and techniques of assisted reproduction and IVF. Along with the latter, increases the occurrence of dangerous and sometimes life-threatening medical complications, which demand a high degree of clinical suspicion in order to be timely diagnosed and properly treated. We present an extremely rare case of simultaneous intrauterine and bilateral ectopic tubal pregnancy, in which the delay in diagnosis lead to a life-threatening rupture and an emergent laparotomy, several days after a first intrauterine intervention had been performed elsewhere. The patient, 38 years old, without any previous medical history but subfertility, was transferred with an ambulance to our Obstetric Emergency department, hemodynamically unstable, with a clinical presentation of acute abdomen and hemoperitoneum. A week ago, she had been submitted to a suction dilation and curettage (D\&C) procedure under anesthesia, in a private centre of our city, due to missed miscarriage of a 7 weeks intrauterine pregnancy, which had been achieved by IVF. A transfer of 3 embryos was reported. Since $\beta$ HCG was high (approx 20000), our initial clinical diagnosis was that of a heterotopic pregnancy, with rupture of supposed tubal gestational sac, which obviously had not been noticed previously by the doctor who performed the D\&C. An ultrasound scan, not thoroughly performed on the way to the operating room, indicated as most possible site of the ectopic sac the ampullary portion of the right tube. Patient's instability and the need for urgent intervention to save her life, made us perform an open laparotomy instead of laparoscopy which in our restricted curve is more time-consuming. Indeed, a rupture of a right ampullary ectopic gestational sac was identified and right salpingectomy was performed. After controlling hemorrhage and washing away the blood and clots from peritoneal cavity, surprisingly a second intact sac in the ampullary of the contralateral tube was identified and then excised. Patient was transfused intra- and post- operatively with 4 units of RBC and transferred to the ICU, from which she was discharged one day after in a very good condition.

In conclusion, the possibility of heterotopic pregnancy, or at its extreme, the co-existence of intrauterine and bilateral tubal triplet pregnancy, even so rare, must be kept in mind in cases where a sonographically confirmed intrauterine pregnancy result in abortion, especially if an IVF cycle with transfer of more than one embryo has been preceded. A delay in proper diagnosis may put a woman's life at risk, as it can result in ineffective treatment and multiple surgical interventions, which may have to be performed on an emergency basis. 


\section{E1452 - ILEAL ATRESIA AFTER FETOSCOPIC LASER PHOTOCOAGULATION FOR TWIN TO TWIN TRANSFUSION SYNDROME}

Anık A. ${ }^{1}$, Akcan B. A. ${ }^{1}$, Çulcuoğlu İ. ${ }^{2}$, Erdem O. A. ${ }^{3}$, Özkıssacık K. S. ${ }^{3}$, Türkmen K. M. ${ }^{1}$

Aydin Adnan Menderes University, Neonatology, Aydin-Turkey ${ }^{1}$

Aydin Adnan Menderes University, Pediatrics, Aydin-Turkey ${ }^{2}$

Aydin Adnan Menderes University, Pediatric Surgery, Aydin-Turkey ${ }^{3}$

Twin to twin transfusion syndrome (TTTS) is an important cause of perinatal morbidity and mortality that seen in $10 \%$ of monochorionic pregnancies. Fetoscopic laser photocoagulation is a treatment modality used in these cases. In recent years, cases with central nervous system, extremity and intestinal system damage such as necrotizing enterocolitis and intestinal atresia have been reported following laser photocoagulation. In this case report, we report a newborn baby with ileal atresia which developed after laser photocoagulation therapy. A male newborn was born at 33 weeks monochorionic diamniotic gestation via cesarean to a 32-year-old woman. His birthweight was $2250 \mathrm{~g}$ and Apgar scores were 8 and 9 at 1 and 5 minutes, respectively. He admitted to our clinic for tachypnea and abdominal distention. Mother's medical history revealed that laser photocoagulation was performed for Quintero stage 1 TTTS at 18 weeks of gestation. Antenatal magnetic resonance imaging (MRI) showed diffuse cystic leukoencephalomalacia in the left cerebral hemispheres and intraabdominal free fluid. Abdominal distention increased at 24th hour and free air was shown under the diaphragm on direct X-ray. Laparotomy revealed intestinal atresia and perforation at a distance of $30 \mathrm{~cm}$ to the cecum. Ileum resection and primary anastomosis was performed. Atretic intestinal segment was seen in the pathology. Cranial MRI showed extensive volume loss in the left cerebral hemisphere and areas of periventricular cystic encephalomalacia. During follow-up, no growth was observed in head circumference. The patient was fed with total enteral breast milk without any problem and was discharged at the 37th week of corrected age.

The aim of this case report is to emphasize that intestinal complications should be kept in mind in the presence of isolated intra-abdominal free fluid in the utero after laser photocoagulation. In monochorionic twins, mesenteric ischemia due to hypoperfusion and hyperviscosity due to vascular anastomosis or thromboembolism after laser photocogulation may play a role in small bowel atresia. 


\section{Obstetrics - Fetal interventions}

\section{E1094 - ACUPUNCTURE AS AN ALTERNATIVE TECHNIQUE IN ESTABLISHING UTERINE CONTRACTIONS IN CONTRACTION STRESS TEST A RANDOMIZED CONTROLLED TRIAL}

Rivera L. ${ }^{1}$, Holgado P. ${ }^{1}$, Tolentino P. ${ }^{1}$, Arcangel C. ${ }^{2}$

University of Santo Tomas, Dept.of Obstetrics and Gynecology, Manila-Philippines ${ }^{1}$

University Of Santo Tomas, Dept. of Anersthesia, Manila-Philippines ${ }^{2}$

\section{Objective}

This study aims to compare contractions produced by acupuncture technique from that of the conventional technique using oxytocin to determine if acupuncture can be an alternative method in establishing uterine contractions in CST.

\section{Methods}

This is a randomized controlled trial employed in fifty-four (54) term high risk pregnancies who were randomized into two groups: 27 patients in the acupuncture group and 27 patients in the oxytocin (control group). Acupuncture needles were applied bilaterally at two loci, Sanyinjiao (spleen 6) and Hegu (Large Intestine 4), to produce the desirable contractions that are interpretable for a CST.

\section{Results}

Subjects who received acupuncture had greater intensity $(\mathrm{p}=0.551)$ and significant longer duration $(p=0.001)$ of uterine contractions than the oxytocin group. However, there was a significant shorter interval of uterine contractions after oxytocin treatment $(p=0.013)$ than acupuncture. Furthermore, subjects who were in the acupuncture group obtained initial uterine contractions ( 5.29 versus 10.62 minutes; $\mathrm{p}=0.000$ ) and achieved desirable uterine contractions (14 versus 30.89 minutes; $\mathrm{p}=0.001$ ) faster than oxytocin. There is shorter waiting time for disappearance of the contractions in the acupuncture group than in oxytocin group (36.70 versus 57.74; $\mathrm{p}=0.000$ ). One subject in the acupuncture group experienced minor bleeding at the needling site and 2 subjects complained of pain from needling. During the conduct of the study, it revealed that subjects in the acupuncture group spent less than in the oxytocin group due to use of lesser materials than that of the conventional method.

\section{Conclusion}

Application of acupuncture in Spleen 6 (Sanyinjiao SP6) and Large Intestine 4 (Hegu LI4) is effective in initiating and inducing uterine contractions. Acupuncture technique when compared to the conventional method using oxytocin, produces stronger and longer contractions. Furthermore, this study showed that contraction stress test can be completed in a shorter time thru acupuncture technique as it shows shorter mean time to achieve initial and adequate contractions thru this technique. Contractions also disappear in a much shorter time in acupuncture technique than in oxytocin group hence ideal for outpatient setting. The adverse effects seen in this study were mild and transient. Cost to perform a contraction stress test using the acupuncture technique is significantly lower than that of the conventional method using oxytocin. Acupuncture should be considered as an alternative technique for contraction stress test as this is, simple, practical, cheap, and safe for the women and her infants. 
E1218 - CANCER OF UNKNOWN PRIMARY SITE DIAGNOSED IN PREGNANCY

Barrenetxea J. ${ }^{1}$, Zabaleta I. ${ }^{1}$, Pedroarena I. ${ }^{1}$, Pérez B. ${ }^{1}$, García S. ${ }^{1}$, Urtasun M. ${ }^{1}$, Abian N. ${ }^{2}$, Gastón B. ${ }^{1}$, Ruiz M. ${ }^{1}$, Bazán M. ${ }^{1}$

Complejo Hospitalario De Navarra, Obstetrics, Pamplona-Spain ${ }^{1}$

Hospital Reina Sofia, Obstetrics, Amorebieta-Spain ${ }^{2}$

Cancer of unknown primary (CUP) is diagnosed in patients who have metastatic cancer, but have no anatomic primary site identified. Adenocarcinomas of unknown primary site comprise approximately 70 percent of CUPs and the most frequently identified sites are lung, pancreas, hepatobiliary tree, and kidney, together accounting for approximately two-thirds of cases.

Our case is a 32 years old woman who went to emergencies due to hematemesis in the second trimester of pregnancy. The woman had two previous at term vaginal deliveries, and was in the 29th week of gestation when the hematemesis occurred. During the exploration an abdominal periumbilical mass was discovered. A CT was performed and a 9x7cm nodule was described, other than that a thickening of the omentum and a thickening of the stomach wall was described.

The nodule was biopsied, and a seal ring cells adenocarcinoma was diagnosed, being the primary tumor in the stomach. Due te the gestational weeks and de diagnosis of a metastatic disease the case was presented in a multidisciplinar committee and the decision was to continue with the pregnancy after explaining the diagnosis and prognosis to the patient. At $37+2$ weeks of gestation induction of labour was scheduled, because of a new episode of hematemesis. PGE2 and Oxitocine was used to induce labour, and an eutocic birth was possible. Ten days after delivery a chemotherapy treatment began. During pregnancy different situations and pathologies can occur being cancer one of those. A rapid diagnosis and a multidisciplinar work group is the key to try to give the best possible solutions to these situations. The moment where the neoplasia is diagnosed is crucial also to determine whether it is better to continue with the pregnancy or to stop it in order to give to the pregnant women the right treatment. 
E1319 - COMPARE THE EFFECT OF ADMINISTRATION OF SERUM $13 \& 23$ RINGER LACTATE \& NORMAL SALINE ON THE DURATION OF LABOR THE RATE OF CESAREAN SECTION SERUM BILIRUBIN\& GLUCOSE LEVELS

Momeni M. ${ }^{1}$, Haghighi L. ${ }^{1}$, Shirazi H. ${ }^{2}$, Tajikrostami F. ${ }^{3}$

Iran Universitiy of Medical Science, Obstetrics \& Gynecology, Tehran-Iran ${ }^{1}$

Iran Universitiy of Medical Science, Medicine, Tehran-Iran ${ }^{2}$

Iran Universitiy of Medical Science, Internal Medicine, Tehran-Iran ${ }^{3}$

\section{Objective}

Undoubtedly, due to the presence of limitations in the active phase of labor (including pain), the need for maternal venipuncture is felt in this phase. Of course Many studies have proven the need for maternal hydration in this phase; Venous hydration is one of the effective factors during labor, However, attention has not been paid to the type of fluid received and the effects of these interventions on the recipient's embryo. The point that is important in this regard is the effect of this intervention on neonatal hypoglycemia and hyperbilirubinemia, Therefore, with regard to the above mentioned cases, we decided to compare the effect of administration of serum $3 / 1$ and $2 / 3$, ringer lactate and normal saline on the duration of labor; the rate of cesarean section; serum bilirubin and glucose levels; and PH of umbilical cord blood In nulliparous women undergoing childbirth induction.

\section{Methods}

This study was performed as a clinical trial study on women referred to the maternity ward of Rasoul Hospital in Tehran, Akbar Abadi Hospital in Tehran, which had criteria for entering the study.

In this study, the same number of G1 and bishop score 4 pregnant women who had indications of termination of pregnancy were included ; In the first group, normal saline was injected during labor and the second group was serum $3 / 1 / 3 / 2$ and the third group was ringer lactate $125 \mathrm{cc} / \mathrm{hr}$ for hydration.

In this study, after data collection, the information entered into the software SPSS; At first, the data were normalized using the Kolmogorov-Smirnov test.Then, in parametric mode, Chisquare, t-test, paired t-test and ANOVA were used for data analysis and in Mann-Whitney, McNemar, Kruskal-Wallis and Wilcoxon tests in parametric bread condition.

\section{Results}

Out of the 350 patients who ultimately remained at the end of the study in this clinical trial Data after normalization and using the Kolmogorov-Smirnov test.Then, in parametric mode, Chi-square, t-test, paired t-test and ANOVA were used for data analysis and in MannWhitney, McNemar, Kruskal-Wallis and Wilcoxon tests in parametric bread condition.

\section{Conclusion}

Ultimately, the results of this clinical trial differed significantly from other similar studies that the outcome of this trial could make it easier to make clinical decisions. 


\section{Obstetrics - Fetal MRI}

\section{E1285 - EVALUATION OF FETAL URETEROCELE A CASE REPORT}

Şal H. ${ }^{1}, \underline{\text { Özalp } M .^{1}}{ }^{1}$, Güvendağ Güven E. ${ }^{1}$

Karadeniz Technical University, Department of Obstetrics and Gynecology, Trabzon-Turkey ${ }^{1}$

Ureteroceles are cystic dilatations of intravesical submucosal ureter. Most cases are associated with complete ureteral and renal duplication and are often associated with ureterohydronephrosis. The incidence was reported to be $1 / 500$.

We report a case of fetal ureterocele diagnosed by prenatal ultrasonography. 32-year-old G1 P0, 22 weeks 6 days dicorionic-diamniotic pregnancy was referred to us by the patient due to pelviectasis. The patient's ultrasonography revealed bilateral pelviectasis, right ureterocele, and bladder distension in the right inferior fetus. Her gender was female. In the foreground, right fetal ureterocele was suspected. The upper left fetus was normal. Fetal MRI was performed for further evaluation. MRI revealed bladder outlet obstruction in the right inferior fetus and reported as right ureterocele.(Figure 1)(Figure 2) She was consulted with pediatric urology for the diagnosis of ureterocele. The patient was followed up with a multidisciplinary approach and the patient was delivered by cesarean section 36 weeks and 4 days old according to the last menstrual period. She was operated by pediatric surgery after delivery. Infant and mother were excised.

It was described by Leshnew in 1921 as a dilatation of intravesical submucosal ureter. It was classified into two groups as intravesical and ectopic by the American pediatric association. In the etiopathology, the early release of the Chwalla membrane during the embryogenesis of the urogenital sinus is accused. In the development of ureteral access, ureterocele formation with orifice not completely punctured is explained.

Duplication is accompanied by $80 \%$ of ureteroceles and $60 \%$ of them are ectopic. Intravesical ureterocele are more common in cases without duplication. In the prenatal period, megasistis, pelviectasis, hydronephrosis and oligohydramnios may be used. In postanatal period, these cases is present with cystitis and pyelonephritis. Although it is usually found incidentally, its treatment can be done in intrauterine or postnatal period. Laser incision and decompression, balloon catheterization and ureterocele cutting can be performed in appropriate cases in intrauterine period. In postnatal period, endoscopic approach, bladder reconstruction and in rare cases conservative treatment can be applied.

Prenatal diagnosis of ureterocele is important for pyelonephritis following pregnancy followup and postnatal follow-up. In cases of severe oligohydramnios and megasistis, termination options may be offered in the early stage and intrauterine treatments should be applied on the viability limit. In mild and moderate cases should be followed with pediatric urology, neonatology and a common approach should be followed in terms of postnatal treatment. 
E1413 - CESAREAN SCAR PREGNANCY THE TREATS OF THE FUTURE THE DIAGNOSIS TREATMENT AND FOLLOW UP OF. CASE REPORTS.

Bursac D. ${ }^{1}$, Heged M. ${ }^{2}$, Lucic D. ${ }^{1}$, Zegarac Z. ${ }^{1}$, Milas M. ${ }^{1}$, Duic Z. ${ }^{1}$

University Hospital Merkur, Obstetrics and Gynecology, Zagreb-Croatia ${ }^{1}$

Genereral Hospital, Obstetrics and Gynecology, Bjelovar-Croatia ${ }^{2}$

Cesarean scar pregnancy (CSP) is a rare condition that occurs when the pregnancy implants in a Cesarean scar. Implantation of a pregnancy within a Caesarean fibrous tissue scar is considered to be the rarest form of ectopic pregnancy and a life-threatening condition. The incidence of CSP has been estimated to range from 1/1800-1/2500 of all Cesarean delivery (CD) performed. The diagnosis and treatment of CSP is challenging. An early diagnosis and a proper management are fundamental to prevent maternal complications. The trend toward an increasing rate of $\mathrm{CD}$ is reported worldwide. A previous $\mathrm{CD}$ increases the risk for a pathologically adherent placenta (accreta, increta, and percreta) and the magnitude of risk increases with each additional CD. Similar risks were reported for CSP. A particular complication of a pregnancy after CD is the implantation of the gestational sac in the hysterotomy scar, known as a "cesarean scar pregnancy" (CSP). The diagnosis is often difficult, and a false-negative diagnosis may result in major complications, including a hysterectomy. The diagnosis is based on finding a gestational sac at the site of the previous $\mathrm{CD}$ in the presence of an empty uterine cavity and cervix, as well as a thin myometrium adjacent to the bladder. Different diagnostic, radiological imaging methods, and management options have been proposed. The optimal management remains to be determined. If the patient presents with a uterine rupture or major bleeding, surgery is unavoidable. Management of diagnosed but stable patients represents a challenge. The follow-up of the patients is described. The objective of this paper was to evaluate the diagnostic method, treatments, and long-term follow-up of CSP. In this article, we describe the intramuscularly use of methotrexate (MTX) as a simple and effective office-based treatment in one patient, and second line chemotherapy with Actinomycin D, Vepesid, Methotrexate and Leucovorin (EMA/CO protocol) in other patients with CSP where MTX therapy give no results. To determinate the diagnosis, we used primarily ultrasound, but in one patient we also did magnetic resonance imaging (MRI). Patients were regularly followed up to negative results. After the treatment was completed by a proven therapy, one of the patients successfully gave birth twice by vaginal delivery; first time after 12 months, and second time after 24 months after therapy. 


\section{Neonatology - Neonatal sepsis}

\section{E1071 - DIAGNOSTIC VALUE OF SERUM AMYLOID A IN EARLY ONSET NEONATAL SEPSIS \\ Ozkan H. ${ }^{1}$, Dorum B. ${ }^{1}$, Cakir S. ${ }^{1}$, Koksal N. ${ }^{1}$, Celebi S. ${ }^{2}$}

Bursa Uludag University Faculty of Medicine, Division of Neonatology, Department of Pediatrics, Bursa-Turkey ${ }^{1}$

Bursa Uludag University Faculty of Medicine, Division of Pediatric Infectious Diseases, Bursa-Turkey $^{2}$

\section{Objective}

Early-onset sepsis (EOS) maintains one of the most important reasons for morbidity and mortality in the neonatal period. To reduce this, early diagnosis and early treatment are critical. The lack of a precise and quick marker to distinguish sepsis from non-infectious diseases is an important challenge. In this study, it was aimed to determine the cut-off value for SAA in predicting early-onset neonatal sepsis with high specificity and sensitivity.

\section{Methods}

This prospective study was carried out in a neonatal intensive care unit of a tertiary hospital. Term and preterm newborns hospitalized between the years 2014 and 2017 for suspected early-onset neonatal sepsis were included in the study. White blood cell count (WBC), platelet count (PLT), C- reactive protein (CRP), procalcitonin (PCT), and serum amyloid A (SAA), values studied at admission and at the 24th and 48th hours of admission, were recorded. The results of overall blood cultures taken in the first 72 hours were evaluated.

\section{Results}

There were a total of 519 newborns were included in the study; 67 in proven sepsis group, 195 in clinical sepsis group and 257 in the control group. Birth weight of the study population ranged between $590 \mathrm{~g}$ and $4760 \mathrm{~g}$ and gestational age was 24-41 weeks. No difference was determined between the demographic characteristics of the newborns in each of the three groups. The three groups were not different in terms of CRP and PCT values at admission. However, SAA value at admission was significantly higher in the cases diagnosed with sepsis as compared to the control group.

\section{Conclusion}

SAA is a reliable diagnostic marker for EOS and has higher sensitivity at symptom onset or in the first hours after birth as compared to CRP and PCT. 


\title{
E1184 - ACCURACY OF CEREBROSPINAL FLUID WHITE BLOOD CELL COUNT GLUCOSE AND PROTEIN IN RAPID DIAGNOSIS OF MENINGITIS IN NEONATES AND YOUNG INFANTS LESS THAN 90 DAYS OLD
}

\author{
Anne R. ${ }^{1}$, Dutta S. ${ }^{1}$, Aggarwal A. ${ }^{2}$, Chadha N. ${ }^{3}$, Kumar P.
}

Post Graduate Institute of Medical Education and Research, Neonatology, Kozhikode-India ${ }^{1}$

Post Graduate Institute of Medical Education and Research, Pulmonary Medicine, Chandigarh- $^{2}$

Post Graduate Institute of Medical Education and Research, Senior Library Assistant, Chandigarh- ${ }^{3}$

\section{Objective}

Rapid diagnostic tests (RDTs) on cerebrospinal fluid (CSF) - white blood cell count (WBCC), glucose, and protein - are widely utilized to diagnose neonatal meningitis. No systematic review and meta-analysis (SRMA) on the diagnostic accuracy of these RDTs is published.

\section{Methods}

We performed an SRMA on studies (January 1, 1950- December 31, 2016) that assessed the accuracy of CSF WBCC, glucose and/or protein (index tests) in diagnosing meningitis among neonates and infants aged $<90$ days. Meningitis was defined as positive reference standard test [CSF culture or gram stain or polymerase chain reaction (PCR)]. We followed standard Cochrane Handbook methodology. We used Stata-14 for analysis. We included all study designs, where the authors had either published data showing cross-classification of index test outcome and disease status, or provided these data on request. We excluded studies on welllooking infants subjected to CSF examination; which had reference standard or index tests other than those specified; published in non-English languages and conference abstracts. We pooled studies for sensitivity, specificity, likelihood ratios (LR) and diagnostic odds ratios (DOR) only if their threshold values were within pre-specified ranges: $19-25 / \mathrm{mm} 3$ in term and $25-29 / \mathrm{mm} 3$ in preterm neonates, $30-40 \mathrm{mg} / \mathrm{dl}$, and $120-170 \mathrm{mg} / \mathrm{dl}$ for term and 150-170 $\mathrm{mg} / \mathrm{dl}$ in preterm neonates for WBCC, glucose and protein respectively.

\section{Results}

From 13,211 titles and abstracts, after excluding duplicates and irrelevant studies, we selected 1023 full-text articles for review; further excluded 1003 based on full-text and included 20 studies for meta-analysis [WBCC: $n=29,906$ (18 studies); glucose: $n=8,352$ for glucose (10 studies); and protein: $\mathrm{n}=8,538$ (11 studies)]. Area under Hierarchical Summary Receiver Operator Characteristic (HSROC) curves of CSF WBCC, glucose and protein were $0 \cdot 89$ [95\% confidence interval (CI): 0.86, 0.91], 0.68 (95\% CI: 0.64, 0.72), and 0.71 (95\% CI: 0.67, $0.75)$ respectively. The pooled sensitivity of WBCC ( $n=9,191,8$ studies), glucose ( $n=1,370,8$ studies) and protein ( $\mathrm{n}=8,419,8$ studies) was 77\% (95\% CI: 69\%, 84\%), 62\% (95\% CI: 43\%, $78 \%)$, and $62 \%$ (95\% CI: 50\%, 73\%) respectively. The pooled specificity was $77 \%$ (95\% CI: 50\%, 92\%), 73\% (95\% CI: 38\%, 92\%) and 92\% (95\% CI: 79\%, 97\%) respectively. The pooled LR+ were 3.3 (95\% CI: 1.4, 8.1), 2.3 (95\% CI: 0.8, 6.9) and 7.8 (95\% CI: 2.8, 21.7) respectively. The pooled LR- were: 0.30 (95\% CI: $0.22,0.41), 0.52$ (95\% CI: $0.28,0.96)$ and 0.41 (95\% CI: 0.30, 0.56). The pooled DOR were 11 (95\% CI: 4, 32), 4 (95\% CI: 1, 23) and 19 (95\% CI: 6, 62) respectively. On meta-regression, study design, year of publication, diagnostic threshold and reference standard used were not statistically significant.

\section{Conclusion}

Overall, CSF WBCC had the best ability to diagnose meningitis in neonates and young infants, followed by protein and glucose. On pooling studies with threshold values within a 
pre-specified range of commonly used cut-off values, the pooled estimates of sensitivity, specificity, LRs and DOR were sub-optimal.

Our study was not funded by any source.

PROSPERO registration number: CRD42017060045. 
E1337 - ADVANTAGES OF THE CAESAREAN SECTION IN THE SPINAL ANAESTHESIA

Cizmovic S. ${ }^{1}$, Grubac S. ${ }^{1}$, Glusac M. ${ }^{1}$, Bozovic R. ${ }^{1}$, Pjescic M. ${ }^{2}$, Mijuskovic M. ${ }^{2}$, Jovanovic V. ${ }^{2}$

General Hospital, Accoucheur Department, Niksic-Montenegro ${ }^{1}$ General Hospital, Anaesthesiology, Niksic-Montenegro ${ }^{2}$

\section{Introduction}

Caesarean section is surgical (accoucheur)operation in which the baby is born through the incision of the abdominal wall and uterus. It may be recommended for almost any obstretic complication, implying a threat to mother or baby.

Anaesthesiology is the branch of medicine concerned with anaesthesia and anaesthetics. It is the medical specialty concerned with the total perioperative care of patients before, during and after the surgery. It encompasses anaesthesia, intensive care medicine, critical emergency medicine and pain medicine. Anaesthesia for the Caesarean section can be general and regional (spinal, epidural, and combined spinal-epidural).

General-endotracheally anaesthesia was the only possible anaesthetical way of birthing center during a long period of time. In the last century it started to stand up for the other regional anaesthetical techniqes. Regional anaesthesia made a great progress in highly developed countries. From all regional techniques for the Caeserean section, the spinal is used quite often.

The advantage of regional anaesthesia over the general is that the numerous complications are very rare, and that the mother can see her baby right away after bits birth.

\section{Objective}

-to determine the percentage of Caesarean section, compared to the region -to determine the frequency of the Caesarean section in the spinal anaesthesia inside our hospital -to determine the difference in Apgar score between the babies born in the spinal or in the general anaesthesia

\section{Methods}

During the period between the 1.1.2016-31.1.2019 retrospectively have been taken some dates from neonatal protocols. 2317 babies have been born.

\section{Results}

776 (33.49\%) Caesarean sections have been performed. Therof the 652 (84.03\%) Caesarean section were performed in the spinal and $124(15.97 \%)$ in the general anaesthesiology. Apgar score 9/ 10 had 296 (38.14\%) from 776 newborns. In the general anaesthesia Apgar score 9/10 had 21 (16.93\%) newborn.

\section{Conclusio}

The advantages/benefits of the regional anaesthesia over the general are related to the woman in labor and the newborns.

When we talk about the woman in labor those benefits are:

- -reduced danger from aspiration of gizzard content

- Mother can see her baby right away and they can realize skin on skin contact।

- Truancy of unpleasant process of extubation of the woman and awakening from the general anaesthesia followed by abdominal pains 
- Early rousing and preventing thrombo-embolism postoperative complications

- Longer absence of pain and reduced need for analgesics in postoperative rehabilitation process

- Taking food and liquid per os after a few hours after the operation

Babies born in the spinal anaesthesia had higher Apgar score value than those babies who are born using the general anaesthesia. The percentage of the Caesarean section using the spinal anaesthesia inside our hospital is on the higher level than in some other cities in our country and it follows the world trends 
E1425 - DISTRIBUTION OF VIRAL LOWER RESPIRATORY TRACT INFECTIONS IN BABIES BORN AT HIGH ALTITUDE BETWEEN OCTOBER 2017 AND JULY 2018

Tekgündüz K. ${ }^{1}$, Kara M. ${ }^{1}$, Özay M. ${ }^{1}$, Özmen E. ${ }^{2}$

Atatürk University Medical Faculty, Pediatrics, Erzurum-Turkey ${ }^{1}$

Atatürk University Medical Faculty, Microbiology, Erzurum-Turkey ${ }^{2}$

\section{Objective}

Respiratory syncytial virus (RSV) is the most common cause of late-onset viral pneumonia in neonates. Chronic pulmonary disease, congenital heart disease and prematurity are important risk factors for RSV infection. Our country is located at above 2000 meter to sea level. The present study seeks to determine the viral pathogens responsible for lower respiratory tract infections in neonates, and investigates the seasonal distribution of causative agents, particularly RSV, in neonates born at high altitudes.

\section{Methods}

The presence of viral pathogens in nasal swabs obtained from 28 patients who were hospitalized with community-acquired lower respiratory tract infections between October 1 , 2017 and June 31, 2018 was investigated by way of a polymerase chain reaction (PCR).

\section{Results}

A viral agent that may be responsible for lower respiratory tract infections was identified in 25 samples (89.2\%). Respiratory syncytial virus (RSV) was the most common viral agent, being detected in 20 patients (80\%), followed by rhinoviruses, which were detected in two patients (8\%). H1N1 was detected in one sample (4\%), human metapneumovirus was detected in one sample (4\%) and parainfluenza was detected in one sample (4\%). No agents were identified in the respiratory panel of the remaining three patients. Of the patients who tested positive for RSV, 10 (50\%) were born prematurely; two premature babies with chronic pulmonary disease died due to RSV infection; and two patients with RSV infections were started on prophylactic therapy against RSV, and of these, one received a single dose and the other received two doses. A diagnosis of RSV infection was made in one patient (5\%) in December, in six patients (30\%) in January, in five patients (25\%) in February, in seven patients (35\%) in March and in one patient (5\%) in April.

\section{Conclusion}

RSV appears as an important cause of morbidity and mortality in neonates, and particularly in preterm babies. Although preterm babies in the high-risk group receive prophylactic palivizumab therapy in the RSV season, the protection provided by this therapy is not 100 percent. Currently, in our country RSV prophylaxis is administered in five doses from October to March, although the present study detected RSV infections also between December and April. This finding suggests that RSV infections could have a distinctive geographical distribution. 


\section{E1433 - CHALLENGES IN DEFINING EARLY ONSET NEONATAL SEPSIS DIAGNOSIS}

Jekova N. ${ }^{1}$, Shopova E. ${ }^{2}$

University Hospital in Obstetrics and Gynecology "Maicin Dom, Neonatology, Sofia

Bulgaria $^{1}$

University Hospital in Obstetrics and Gynecology "Maichin Dom, Microbiology, "Sofia-Bulgaria ${ }^{2}$

\section{Objective}

Early - onset neonatal sepsis (EONS) remains a significant global health problem and potentially fatal illness. Despite extensive study, identifying at-risk neonates remains challenging, especially if they are initially well appearing. Presenting clinical symptoms are unspecific and both sensitivity and positive predictive value of laboratory tests at onset of symptoms are not optimal. A positive microbial blood culture is the gold standard to define a neonatal sepsis. However, the incidence of culture confirmed early-onset sepsis is rather low, around $0.4-0.8 / 1000$ term infants in high-income countries. This leads to overuse of broadspectrum antibiotics for treatment of suspected sepsis with all negative sequences for the survivors due to a lack of a clear definition and a robust gold standard of the diagnosis neonatal sepsis. The objective of this clinical report is to summarize the main challenges in defining the diagnosis of a culture-negative neonatal sepsis with a focus on EONS.

\section{Methods}

Our report is based on the data of the most recent studies and the state-of-the art clinical practices in the leading neonatal centers.

\section{Results}

Neonatal sepsis is a dynamic and complex condition. A static definition of clinical symptoms reflecting organ dysfunction at a single point (disease onset) is too unspecific to define EONS and guide antibiotic therapy. A combination of risk factors, symptoms at disease onset combined with development of symptoms upon evaluation after 36-48 h has proved to be a reasonable strategy for defining the diagnosis. Moreover, the biochemical picture of neonatal sepsis changes over the first days after antibiotics has been commenced and the kinetics of inflammatory biomarkers (reflecting a host inflammatory response) should also be included in a future neonatal sepsis definition. Appropriate blood culture diagnostics requiring a minimum $1 \mathrm{ml}$ blood obtained before commencing antibiotic treatment substantially reduces the number of cases with the culture-negative sepsis.

\section{Conclusion}

Clinical guidelines for diagnostic assessment and management of EONS should be based on a robust and pragmatic neonatal sepsis definition. 
E1436 - CLINICAL CHORIOAMNIONITIS AND HISTOLOGIC PLACENTAL INFLAMMATION ASSOCIATION WITH EARLY NEONATAL SEPSIS

Lithoxopoulou M. ${ }^{1}$, Rallis D. ${ }^{1}$, Pervana . $^{2}$, Tsakalidis C. ${ }^{1}$, Karagianni P. ${ }^{1}$, Hatziioannidis E. ${ }^{1}$, Babatseva E. ${ }^{1}$, Soubasi V. ${ }^{1}$

Aristotle University of Thessaloniki, Papageorgiou Hospital, 2nd Nicu and Neonatology

Department, Thessaloniki-Greece ${ }^{1}$

Aristotle University Papageorgiou Hospital, Department of Pathology, Thessaloniki-Greece ${ }^{2}$

\section{Objectives}

The gold standard for diagnosing chorioamnionitis is placental histological examination, however the delay for the final results hinder its use as an indicator to guide early postnatal therapy. Neonates whose mothers are diagnosed with chorioamnionitis should be treated with empiric antibiotics pending blood culture results. Accordingly, many uninfected neonates are exposed to systemic antibiotics. Our objective was to estimate the rate of confirmed histologic chorioamnionitis (HCA) in the presence of clinically chorioamnionitis, and to evaluate the correlation of the HCA with the inflammatory markers of early onset sepsis (EOS).

\section{Methods}

We retrospectively reviewed all neonates admitted to the 2nd Neonatal Unit of Aristotle University of Thessaloniki during 2017-2018 with suspected clinical chorioamnionitis. We recorded the clinical evidence of chorioamnionitis and the histologic report of placenta, the clinical signs and inflammatory markers related to EOS, and finally, we used the Kaiser calculator, applied to neonates $\geq 34$ weeks' gestation for the estimation of the sepsis clinical illness score (SCIS).

\section{Results}

From 266 neonates admitted with the suspicion of clinical chorioamnionitis, 81 (30\%) had HCA. Histologic chorioamnionitis was associated with earlier gestational age ( $31.8 \pm 4$ versus $33.3 \pm 4$ weeks, $\mathrm{p}=0.004)$ and lower birth weight $(1826 \pm 840$ versus $2092 \pm 849 \mathrm{~g}, \mathrm{p}=0.019)$. Maternal fever, prolonged rupture of membranes, foul-smell amniotic fluid and fetal tachycardia were prominent in pregnancies associated with histological chorioamnionitis. Neonates with histological chorioamnionitis had significantly higher rate of clinical symptoms indicating early sepsis ( $31 \%$ versus $15 \%, \mathrm{p}=0.004)$, higher CRP at birth $(1.4 \pm 1.5$ versus $0.37 \pm 0.3 \mathrm{mg} / \mathrm{dL}, \mathrm{p}<0.001)$ and at 24 hours $(2.2 \pm 2.4$ versus $0.4 \pm 0.6 \mathrm{mg} / \mathrm{dL}, \mathrm{p}<0.001)$, and higher rate of positive blood culture $(25 \%$ versus $3 \%, \mathrm{p}<0.001)$. When histologic chorioamnionitis was present, the administration of maternal antibiotics was related to lower CRP at birth and at 24 hours $(\mathrm{p}<0.001)$.

The SCIS was significantly higher in neonates with HCA (2.96 versus $0.02, \mathrm{p}<0.001$ ); those neonates received a full course of antibiotics in $46 \%$ compared to $4 \%$ of neonates with absent HCA $(\mathrm{p}<0.001)$.

For detecting HCA, the sensitivity and specificity of positive CRP at birth was $64 \%$ and $81 \%$, while for CRP at 24 hours was $78 \%$ and $76 \%$, respectively.

\section{Conclusion}

Clinical chorioamnionitis was not supported by histological evidence in $70 \%$ of cases. HCA was associated with increased rates of maternal and neonatal symptoms, higher inflammatory markers, and significant response to the administration of maternal antibiotics. Positive CRP at birth and at 24 hours of age had significant sensitivity and specificity for detecting HCA and could be used for ruling-out EOS and discontinuing antibiotics. 


\section{E1453 - ILIOPSOAS ABSCESS IN A NEWBORN; TREATMENT WITH PERCUTANEOUS DRAINAGE CATHETER \\ Kara M. $^{1}$, Tekgündüz K. ${ }^{1}$, Eren S. ${ }^{2}$}

Ataturk University Medical Faculty, Pediatrics, Erzurum-Turkey ${ }^{1}$

Ataturk University Medical Faculty, Radiology, Erzurum-Turkey ${ }^{2}$

\section{Introduction}

Iliopsoas abscess (IPA) is a rarely seen in children, especially in newborn infants. It is classified as primary of secondary. Etiology is unknown is primary IPA and it develops as a result of hematogenic or lymphatic spread of microorganisms from a focus of unknown origin. Psoas muscle has a close neighborliness with retroperitoneal organs, gastrointestinal system and the muscle-skeletal system. So, psoas muscle may be affected secondarily during the infectious diseases of these systems. In present study we aimed to report treatment of a primary IPA by percutaneous drainage catheter in a newborn infant.

\section{Case}

A newborn infant who was a product of a 38- weeks gestation of a 36-years mother (5th gestation, birth weight was $2960 \mathrm{gr}$ ) had been treated for hyperbilirubinemia of newborn in a state hospital. Two days after discharge, he had developed swelling and redness from the left inguinal region to the left knee.

The patient was admitted to our clinic on postnatal 17th day. Physical examination revealed swelling and redness from the left inguinal region extending to the left knee, and hip movements were painful. MRI showed an abscess $(24 \mathrm{X} 23 \mathrm{~mm})$ on left iliopsoas region. Interventional radiology team implanted percutaneous drainage catheter. Cavity was washed with isotonic solution daily. Empirically meropenem and vancomycin was administered. Staphylococcus aureus was isolated from aspirate culture, and it was sensitive to administered antibiotics. On the 4th week of treatment, CT revealed the complete resolution of abscess. Drainage catheter removed and treatment completed to 6 weeks.

\section{Conclusion}

Iliopsoas abscess is a purulent retroperitoneal collection in the iliopsoas muscle. Etiologically it may be primary and secondary. As we could not detect an etiological reason our case was accepted as a primary IPA. Previous reports indicate that results of percutaneous drainage are superior to surgical drainage and it is less invasive. By this method, deep IPAs can be treated via a small incision as in our case. 
E1464 - MANAGEMENT OF TERM INFANTS AT RISK FOR EARLY ONSET GBS SEPSIS IN CORELATION WITH MOTHERS GBS SCREENING STATUS AND INTRAPARTUM ANTIBIOTIC EXPOSURE IN ALL NATURAL BIRTH BORN TERM INFANTS

Zdjelar S. ${ }^{1}$, Lakovic G. ${ }^{1}$, Mihajlovic S. ${ }^{2}$, Kubat J. ${ }^{2}$, Nikolic M. ${ }^{2}$

Kbc Dr Dragisa Misovic, Neonatology Department, Belgrade-Serbia ${ }^{1}$

Kbc Dr Dragisa Misovic, Hospital for Gynecology and Obstetrics, Belgrade-Serbia ${ }^{2}$

Early-onset neonatal bacterial sepsis is occurring within the first 7 days. Most infants become symptomatic within 24 hours of birth and the infection is usually result from horizontal and vertical transmission with organisms that colonize the birth canal. GBS rarely can ascend to amniotic fluid colonizing the infant or the infant may become colonized during passage through the birth canal. Maternal GBS colonization in current pregnancy, GBS bacteriuria, a previous infant with invasive GBS disease, prolonged rupture of membranes ( $\geq 18$ hours) and maternal fever (>38 C) are the factors most commonly associated with early-onset GBS sepsis. At present, there is no laboratory test that has sufficient sensitivity to allow clinicians to safely rule out GBS sepsis.

The management of well-appearing at-risk term infants depends on the number of risk factors and whether maternal intrapartal antibiotic prophylaxis for GBS was used. Careful assessment and observation of these at-risk infants are fundamental component of appropriated care. In the year of 2018. we had in our hospital 2247 newborns of which $70.2 \%$ were natural birth born and $82.2 \%$ of them were term infants. More than $90 \%$ of mothers had GBS screening results from 35-37 weeks of pregnancy. 


\section{E1472 - INVASIVE FUNGAL INFECTIONS IN THE NEONATE}

Ben Thabet A. ${ }^{1}$, Abdelkarim R. ${ }^{1}$, Ben Hamad A. ${ }^{1}$, Regaieg C. ${ }^{1}$, Charfi M. ${ }^{2}$, Hamida N. ${ }^{1}$, Bouraoui A. ${ }^{3}$, Gargouri A. ${ }^{1}$

Hedi Chaker Hospital, Neonatology, Sfax-Tunisia ${ }^{1}$

Hedi Chaker Hospital, Neonatolgy, Sfax Tunisia ${ }^{2}$

Hedi Chaker Hospital, Neonatlogy, Sfax-Tunisia ${ }^{3}$

\section{Objective}

Invasive nosocomial fungal infections remain a serious complication of infants's stays in neonatal intensive care units. Candida species are the third most common pathogen in neonatal nosocomial blood stream infections in premature infants, and they are associated with the second highest mortality rate. Preventive measures therefore remain the best means to reduce these infections.

\section{Methods}

This is a retrospective study conducted at the department of neonatology in Hedi Chaker Hospital, in Sfax in Tunisia between january 2005 and december 2018. We analyzed all records of newborns hospitalized during this period and presenting a nosocomial Candida infection was confirmed.

We have retrospectively compiled epidemiological, clinical, biological and evolutionary data from the neonates files included in the study. In addition, all our patients benefited from Transfontanellar ultrasound and abdominal ultrasound in search of visceral involvement.

\section{Results}

6 episodes of fungal infections were confirmed in newborns hospitalized in our resuscitation unit. There where 3 males and 3 females. The average term was $34 \mathrm{GW}+/-2$ weeks. The mean birth weight was $1975 \mathrm{~g}$.

The mean age at the time of suspicion of nosocomial fungal infection was 9 days + /- 7days. In four cases, the infection was suspected after a 7-day stay in the intensive care unit. Five newborns were treated by antibiotic therapy for anterior nosocomial bacterial infection confirmed by blood culture. The main clinical abnormalities observed in infected babies, on the day of diagnosis of nosocomial candida fungal infection were: haemodynamic disorders ( 5 cases), thermal instability, especially fever (4 cases), neonatal seizure and hypotonia (2 cases) and secondary respiratory distress (3 cases). Laboratory findings were non-specific : leukocytosis was observed in 2 cases, leucopenia in 1 case and thrombocytopenia in 2 cases. A positive CRP was observed in only 3 cases.

Candida was isolated in 5 blood culture. 2 were Candida albicans, 2 were Candida glabrata and one case of Candida Tropicalis. Peripheral samples were positive in 2 neonates: (Cerebrospinal fluid and urine). Complementary examinations (Transfontanellar ultrasound, abdominal ultrasound and fundus photography) had shown no deep visceral involvement. All neonates were treated with fungal treatment. The duration of the treatment was 21 days for 3 infants, 15 days for one infant, 10 days for one infant how has negative blood culture. One infant was died due a state of refractory septic shock secondary to sepsis candidiasis. Two children were diagnosed with psychomotor retardation during the follow-up period

\section{Conclusion}

Rates of invasive fungal infection are highest among neonates, especially those of low birthweight. Diagnosis may be delayed because of a nonspecific clinical presentation; difficulty in culturing the organisms. Treatment can involve a long and costly therapeutic 
course. Furthermore, IFI in neonates carries a high mortality and leads to significant neurodevelopment impairment in survivors.

Thus, a prospective cohort study designed to risk stratify neonates would help in the implementation of the proper use of prophylaxis and empirical therapy for invasive candidiasis. 
E1484 - A RARE BACTEREMIA IN IMMUNOCOMPROMISED NEONATE IN NEONATAL INTENSIVE CARE UNIT HERBASPIRILLUM HUTTIENSE

$\underline{\text { Tas M. }}{ }^{1}$, Turkyilmaz C. ${ }^{1}$, Bozyazi G. ${ }^{2}$, Tezer H. ${ }^{3}$, Bas M. ${ }^{1}$, Keles E. ${ }^{1}$, Kenar A. ${ }^{1}$, Deniz M. ${ }^{3}$, Hirfanoglu I. ${ }^{1}$, Onal E. ${ }^{1}$, Ergenekon E. ${ }^{1}$, Koc E. ${ }^{1}$

Gazi University Faculty of Medicine, Neonatal Intensive Care Unit, Ankara-Turkey ${ }^{1}$

Gazi University Faculty of Medicine, Department of Medical Microbiology, Ankara-Turkey ${ }^{2}$

Gazi University Faculty of Medicine, Department of Pediatrics Infectious Diseases, Ankara

Turkey $^{3}$

\section{Introduction}

Herbaspirillum species are gram-negative Betaproteobacteria that inhabit the rhizosphere. They are found in the roots and stems of maize, rice, beans, bananas, sugar cane, pineapple, and other plants. They have been recovered from groundwater and drinking water distribution systems as well. It can colonize the airways of immunocompromised patients causing bacteremia and sepsis. In this case report, we present a case of H.Huttiense bacteremia while high dose steroid was given to a thrombocytopenic neonate born to mother with idiopathic thrombocytopenic purpura (ITP).

\section{Case}

Our patient was born as a G2P2 child from a 28-year-old mother who was followed up for eight years with the diagnosis of chronic ITP. The patient was admitted in the neonatal intensive care unit after platelet cell count was detected $70.000 / \mathrm{mm} 3$ in complete blood count. The mother of the patient was given methylprednisolone $24 \mathrm{mg}$ /day after the first trimester and was given intravenous immune globulin (IVIG) three times during pregnancy. The physical examination was unremarkable. A total of $3 \mathrm{~g} / \mathrm{kg}$ IVIG transfusion was given to the infant. During the follow up, platelet cell counts was not elevated, due to recommendation of pediatric hematology 2 $\mathrm{mg} / \mathrm{kg} /$ day methylprednisolone was started. Transfontanel ultrasound showed $3.5 \times 1.5 \mathrm{~cm}$ intracranial hematoma in the parietooccipital area. On the second day of steroid treatment, the platelet value was found to be $27.000 / \mathrm{mm} 3$ and high dose steroid was recommended. Methylprednisolone was started at $30 \mathrm{mg} / \mathrm{kg} /$ day for three days and then $20 \mathrm{mg} / \mathrm{kg} /$ day for four days. On the third day of high dose steroid, fever was found $38.2{ }^{\circ} \mathrm{C}$. Acute phase reactants and blood culture were studied. Laboratory results revealed CRP: $44.2 \mathrm{mg} / \mathrm{L}$, PCT: $2.57 \mathrm{ng} / \mathrm{mL}$, IL6: 4878 pg/mL, Plt:19.000/mm3 Wbc: 3260/uL, Leu:1550/uL. Lumbar puncture could not be performed due to thrombocytopenia and intracranial hematoma. Vancomycin and piperacillintazobactam antibiotherapy were initiated. High dose steroid treatment was continued. Next day the patient had Gram negative bacilli signal in the blood culture. Herbaspirillum huttiense growth was detected in both first blood culture and confirmed with repeated blood culture using MALDI-TOF-MS (matrix assisted laser desorption ionization-time of flight mass spectrometry) method. Genomic sequence of strain planned to be studied in molecular microbiology laboratory. The susceptibility test show no resistance at any antibiogram and the current treatment was continued. After high dose steroid, platelet values increased to $126.000 / \mathrm{mm}$. Control assays showed regression in acute phases. Antibiotic regimen completed in ten days. In transfontanel ultrasound, hematoma regressed to $23 \times 1,5 \mathrm{~cm}$. The control blood culture of the patient was sterile. The patient was discharged to come to the outpatient clinic. 


\section{Conclusion}

Herbaspirillum Species are capable of causing bacteremia and sepsis in immunocompromised patients. In our case, it is important to highlight the high dose steroid as predisposing factor contributing to the patient's susceptibility to developing this infection. When we examined the literature in the newborn period, we found only one case who had prematurity. The newborn with $\mathrm{H}$. Huttiense infection due to high dose steroid was the first case in the literature. 


\section{Neonatology - Problems of the premature neonate}

\section{E1039 - CORRELATION BETWEEN INTRAUTERINE GROWTH RESTRICTION AND HYPERTENSIVE DISORDERS IN PREGNANCIES WITH IVF PROCEDURES Luta J. $^{1}$, Krasniqi M. ${ }^{2}$}

University of Prishtina, Midwife, Prishtina-Albania ${ }^{1}$ Clinical Center of Kosovo, Gynecology, Prishtina-Albania ${ }^{2}$

\section{Background}

Intrauterine growth restriction (IUGR) refers to a condition in which an unborn baby is smaller than it should be because it is not growing at a normal rate inside the womb. Delayed growth puts the baby at risk of certain health problems during pregnancy, delivery, and after birth. They include low birth weight. The aim of this study was to see how many women that had infants born with IUGR and the correlation between hypertensive disorders and intrauterine growth restriction.

\section{Methods}

A total of 158 patients underwent in vitro fertilization (IVF) treatment to become pregnant. They were chosen based on their previous births, if their infants had pre intrauterine growth restrictions. This was a two year study and took place in Prishtina Kosovo

\section{Results}

Out of the total number of infants born 14 of them (16\%) had intrauterine growth restriction (IUGR). The highest percentage of infants born with intrauterine growth restriction had in the group of pregnant women who exhibited hypertensive disorders during pregnancy by $6(11 \%)$ compared to infants born from the group who did not exhibit these disorders $8(5.2 \%)$, however the difference was not significant $(\mathrm{P}>0.05)$,

\section{Conclusions}

We concluded that at IVF procedures and IUGR (intrauterine growth restriction) is not correlated however women that had signs and symptoms of hypertensive disorders during pregnancy did have infants with IUGR. 


\section{E1070 - THE FIRST 24 HOUR RENAL TISSUE OXYGEN SATURATIONS IN PREMATURE INFANTS CAN PREFER ACUTE KIDNEY INJURY \\ Koksal N. ${ }^{1}$, Ozkan H. ${ }^{1}$, Cetinkaya M. ${ }^{2}$, Dorum B. ${ }^{1}$, Cakir S. ${ }^{1}$}

Bursa Uludag University Faculty of Medicine, Division of Neonatology, Department of Pediatrics, Bursa-Turkey ${ }^{1}$

Health Sciences University, Kanuni Sultan Suleyman Training and Reseaech Hospital, Division of Neonatology, Department of Pediatrics, Istanbul-Turkey ${ }^{2}$

\section{Objectives}

Acute kidney injury (AKI) is frequently seen in premature infants and is important in terms of mortality and morbidity. It is known that decreased renal blood flow has a major role in the etiology of AKI in preterm infants. In this study, we aimed to investigate the role of renal tissue oxygen saturation (RSO2) in predicting the development of AKI in the first 24 hours of life.

\section{Methods}

This prospective study included 100 premature infants with a gestational age of $\leq 32$ weeks. All infants were born with near-infrared spectroscopy (NIRS) and cerebral, renal and mesenteric tissue oxygen saturation was monitored for 24 hours. The infants were followed up for AKI development for 1 week. AKI serum creatinine (Scr) level was defined as $1.3 \mathrm{mg} /$ dl after the first day of life.

\section{Results}

Mean gestational age was $28.7 \pm 2.1$ weeks and birth weight was $1192 \pm 355$ gr. In the first week of life, a total of 29 patients developed AKI during the study period. Sixteen of these cases developed at postnatal 2nd and 3rd day of AKI. Renal RSO2 values in the first 24 hours of life were lower in all hours and this decrease was significant in the first 6 hours.

\section{Conclusion}

In this study, it was found that the low renal RSO2 values in the early hours of life in premature infants may have a role in predicting the early stage of AKI and the importance of monitoring with renal function and NIRS in infants with gestational age $<32$ weeks was shown. 


\title{
E1072 - RISK OF PORTAL VEIN THROMBOSIS IN THE PREMATURE BABIES
}

\author{
Cakir S. ${ }^{1}$, Ozkan H. ${ }^{1}$, Koksal N. ${ }^{1}$, Dorum B. ${ }^{1}$, Sezgin Evim M. ${ }^{2}$
}

Bursa Uludag University Faculty Of Medicine, Division Of Neonatology, Department Of

Pediatrics, Bursa-Turkey ${ }^{1}$ Bursa Uludag University Faculty Of Medicine, Division Of

Pediatric Hematology, Department Of Pediatrics, Bursa-Turkey ${ }^{2}$

\section{Objectives}

In the neonatal period, umbilical venous catheters (UVC) are frequently used to provide vascular access. These catheters have various complications such as mechanical, infectious and thrombosis. The incidence of UVC-induced thrombosis is $65 \%$ in autopsies, $30 \%$ in venography and $1.3-43 \%$ in ultrasound scans. In this study, it was aimed to determine the rates and risks of portal vein thrombosis (PVT) in the neonatal intensive care unit (NICU). In addition, the management of these patients was discussed by reviewing the relevant literature.

\section{Methods}

In this study, infants with a catheter under 32 gestational weeks of NICU in 2016-2018 were examined. Demographic data of the patients, number of days of catheter stay, number of erythrocyte suspension from the catheter, total parenteral nutrition (TPN) intake times, catheter locations, time of thrombosis by doppler ultrasound, treatment methods and durations, thrombosis follow-up and thrombosis tests retrospectively obtained from electronic patient files.

\section{Results}

96 patients were included in the study. Fifty-two percent of the patients were male. The mean gestational age was $29 \pm 2$ weeks (24-32 weeks) and the mean birth weights were $1353 \pm 369$ gr $(630-2210$ gr $)$. PVT was detected in $13.5 \%(n=13)$ of the patients. Five of the PVTs were complete occlusive, and 8 were partial occlusion. There were no differences in terms of birth weight, gestational age, gender, Apgar scores among patients with and without PVT. Maternal preeclampsia rates, rate of low birth weight, sepsis rates, duration of catheter use, number of days of TPN administration, number of erythrocyte suspensions were similar in both groups. There was no difference between the groups in terms of hematological parameters. There was no significant relationship between UVC location and PVT development. In all patients with complete occlusion and in 6 patients with partial thrombosis, low molecular weight heparin treatment was performed on a mean of $31 \pm 13.8$ days and PVT was recanalization between 7-120 days in all patients.

\section{Conclusions}

PVTs that are involved in the etiology of portal hypertension in childhood are mostly asymptomatic in the neonatal period and cannot be clinically recognized. Screening and follow-up of doppler USG in terms of PVT is important for the prevention of long-term complications. 
E1134 - CARBON DIOXIDE FLUCTUATIONS ARE ASSOCIATED WITH CHANGES IN CEREBRAL OXYGENATION IN PREMATURE INFANTS

Ozkan . $^{1}$, Cetinkaya M. ${ }^{2}$, Koksal N. ${ }^{1}$, Dorum B. ${ }^{1}$

Uludag University, Neonatology, Bursa-Turkey ${ }^{1}$

Kanuni Sultan Suleyman Traning and Research Hospital, Neonatology, Istanbul-Turkey ${ }^{2}$

\section{Objective}

To evaluate the effects of carbon dioxide partial pressure changes on cerebral oxygenation.

\section{Methods}

In this prospective study, $\leq 32$ gestational age and $\leq 1500$ grams of infants who received respiratory support were included. The babies were grouped as $<28$ weeks and $\geq 28$ weeks according to gestational age. Cerebral, renal, mesenteric tissue oxygen saturation (RSO2) and fractionated tissue oxygen extractions (FTOE) were recorded simultaneously with blood gas measurement from the first day of life of all infants. Seven hundred PCO2 results of 100 patients were evaluated. PCO2 was defined as $<35 \mathrm{mmHg}$ hypocarbia and $>55 \mathrm{mmHg}$ hypercarbia.

\section{Results}

The median gestational age was 29 weeks and birth weight was 1147 gr. There was a positive correlation between PCO2 levels and cerebral RSO2 in both gestational week groups. Mean cerebral RSO2 was found to be $40 \pm 9.4$ in hypocarbia and $65.4 \pm 9.2$ in hypercarbia in gestational age <28 weeks. In the same group, mesenteric and renal RSO2 values were significantly lower in response to cerebral RSO2 increase during hypercarbia.

\section{Conclusion}

There was a positive correlation between PCO2 levels and cerebral RSO2 in premature infants. Changes in tissue oxygen saturation during hypo-hypercarbia are more evident in infants with gestational age $<28$ weeks. 


\section{E1155 - RESPIRATORY SUPPORT IN PREMATURE INFANTS WITH CONGENITAL INFECTIONS}

Garayeva . $^{1}$, Ibadova T. $^{2}$, Mammadova A. ${ }^{3}$, Huseynova N. ${ }^{1}$

Azerbaijan Medical University, Department of I Children Diseases, Baku-Azerbaijan ${ }^{1}$

Azerbaijan State Advanced Training Institute for Doctors, Department of Pediatrics, Baku

Azerbaijan $^{2}$

Scientific Researches Institute of Pediatrics, Department of Newborns Pathology, BakuAzerbaijan $^{3}$

\section{Introduction}

The pathology of the respiratory system is one of the main causes of high morbidity and mortality in newborns. In the structure of the causes of early neonatal mortality, respiratory distress syndrome takes the third place after birth trauma, intrauterine hypoxia and asphyxia and is $16.4 \%$. According to the WHO (Bryce J., 2005), intrauterine infection takes the first place (19\%) among the infections that cause infant mortality. The introduction of modern treatment technologies into practice, in particular, artificial ventilation of the lungs (ALV), has made it possible to successfully nursing newborns with morphofunctional immaturity, extremely low body mass and profound dysfunction of the respiratory system associated with intrauterine infection.

\section{Objective}

To study the features of respiratory support in the period of early postnatal adaptation in preterm infants with congenital infections

\section{Methods}

A selective study was conducted, which included 48 premature neonates. The average gestation period was 32 (28-35) weeks, body weight - 1830 (1040-2420) g. The examination included premature newborns with a congenital infection who needed respiratory support in the intensive care unit (main group) and 56 term newborns with congenital infections (comparison group).

\section{Results}

The state at birth of all preterm neonates was very difficult due to the development of respiratory distress syndrome RDS. The following methods of respiratory support were used in the main groups of children studied: oxygen therapy with humidified oxygen, CPAP and artificial lung ventilation (ALV). One of the methods of prevention and treatment of respiratory failure in premature newborns, the clinical effectiveness of which has been proven by many studies, is non-invasive ventilation of the lungs by the method of constant positive pressure through the nasal cannulas - nasal CPAP. $10.4 \pm 4.4 \%$ of premature babies with congenital infection and $11.8 \pm 3.1 \%$ of full-term babies needed mechanical ventilation. In preterm infants of the main group, oxygen therapy was performed in $47.9 \pm 7.2 \%$ of cases, in the comparison group of term newborns $-52.7 \pm 4.8 \%$. Non-invasive ventilation of the lungs was performed using CPAP in $15.5 \pm 3.4 \%$ of the children in the comparison group, $29.2 \pm$ $6.6 \%$ in the main group. 


\section{Conclusion}

Thus, in our study, children used various methods of respiratory support in various combinations. The use of modern methods of mechanical ventilation allows minimizing disabling outcomes in premature babies. Newborns with generalized forms of congenital infection are born in asphyxia and need intensive care and resuscitation from the first minutes of life, and most have various cerebral and respiratory disorders that force them to resort to respiratory support procedures both from the first days of early neonatal and throughout neonatal period. 
E1159 - MORTALITY OF VERY PREMATURE INFANTS (ABOUT 565 CASES) IN SFAX TUNISIA

$\underline{\text { Ben Hamad A. }}{ }^{1}$, Ben Thabet A. ${ }^{1}$, Charfi M. ${ }^{1}$, Loukil M. ${ }^{1}$, Regaieg C. ${ }^{1}$, Regaieg R. ${ }^{1}$, Hamida N. ${ }^{1}$, Bouraoui A. ${ }^{1}$, Gargouri A. ${ }^{1}$

University of Sfax, Neonatology, Sfax-Tunisia ${ }^{1}$

\section{Objective}

The maternity of Sfax, is the only level 3 maternity in the whole of southern Tunisia. Advances in neonatology and obstetrics have made possible the survival of neonates with lower gestational ages and birth weights We conducted a retrospective study to evaluate hospital mortality of very premature infants in the neonatology department of Sfax.

\section{Methods}

This is a retrospective descriptive study over a 3-year period from January 2012 to December 2014 including all premature infants born before 33 weeks at the maternity of Sfax and hospitalized in the neonatal unit care.

\section{Results}

A total of 565 premature infants were included in the study. Thus, the incidence of very prematurity compared to the number of newborns in the maternity was $1.85 \%$. We noted around 190 of very premature infants per year. 142 neonates were died. The overall hospital mortality in our study was $25.1 \%$.

These deaths accounted for $37.8 \%$ of all newborns who died in our service during the 3 years of the study. The most significant risk factors for mortality were: low gestational age, especially before 28 weeks (58 \% before this term versus 30\% between 28 and 30 weeks and $12 \%$ above 31 weeks), low birth weight less than $1000 \mathrm{~g}$, male sex and the lack of antenatal corticosteroid therapy.

\section{Conclusion}

The mortality of very premature infants has fallen sharply in recent years. However, the prognosis remains severe in premature infants before 28 weeks. The therapeutic management of these patients is still expensive and difficult in our context. 
E1189 - ETHICAL DECISION MAKING APPROACHES IN NICUS EXPERIENCES AND PRACTICES OF GREEK HEALTHCARE PROFESSIONALS

Daglas $M .^{1}$, Petousi V. ${ }^{2}$, Poulios A. ${ }^{3}$

University of West Attica, Midwifery, Athens-Greece ${ }^{1}$

University of Crete, Sociology, Rethymno-Greece ${ }^{2}$

National Kapodestrian University, Psychology, Athens-Greece ${ }^{3}$

\section{Objective}

International epidemiological data regarding infants born prior to the 24th week of gestation tend to be rather dejecting. The survival rates of those infants do not seem to increase, while no encouraging changes have been recorded regarding the rate of occurrence of related physical or mental disabilities. In the face of the above, a pressing bioethical dilemma is raised: should human life be saved at any cost and with any method (including highly interventional intensive care) irrespective of outcome or should limits be put in place? International literature and research identifies three general approaches of ethical decision making in relation to neonatal intensive care: (a) the "wait until certainty strategy"; intensive care is offered to all newborns; (b) the "statistical prognostic strategy"; the care to be provided is based on statistical probabilities for an unfavorable diagnosis or bad outcome of the incident; and (c) the "individual prognostic strategy"; intensive care is initiated for almost every newborn and reassessed regularly thereafter. Neonatal Intensive Care Units' (NICUs) implemented approach differs among countries and depends on multiple factors (e.g. health care system, resources, culture) prominent among them being the lack of a unified and unambiguous regulatory framework. The objective of this research is to look into the clinical experience of healthcare professionals in Greek NICUs and record the ethical decisionmaking approach they implement when faced with neonatal care dilemmas.

\section{Methods}

Survey research based on the EURONIC project questionnaires (weighted, international) adjusted and translated into Greek was conducted on a sample of 251 health care professionals (71 medical doctors, 98 midwives and 82 nurses) working in 17 public NICUs in Greece. This paper reports partial findings based on healthcare professionals' responses to questions about the most recent clinical event in their NICU for which they had to make a decision (individually or jointly with others) on an ethical dilemma regarding neonatal care.

\section{Results}

Significant prematurity (38.7\%) or diagnosed severe neonatal brain haemorrhage $(23.2 \%)$ were the most frequently identified conditions which gave rise to the following ethical dilemmas: a) continuation or termination of provided intensive care (56.7\%), b) resuscitation after labour (23.9\%) and c) initiation or not of intensive care $(18.3 \%)$. Withholding of provided intensive care was decided in less than half of the cases (45\%). Specifically, either urgent interventions were not applied (24.4\%) or intensive care was not intensified (20.6\%). Initiated intensive care continued normally in almost one third of the cases (34.4\%). Neither resuscitation nor initiation of intensive care was decided in $12.7 \%$ of cases while withdrawal of life support or therapy provided to newborns occurred rarely (3.3\%). 


\section{Conclusion}

Healthcare professionals in Greek public NICUs, for the most part initiate and continue intensive care to all neonates. Nevertheless, withholding rather than withdrawing provided care is preferred for cases in which care is limited. It appears thus, that when faced with ethical dilemmas regarding neonatal care Greek health care professionals tend to prioritize the 'wait until certainty approach' followed by the 'individual prognostic strategy'. 
E1197 - IMPACT OF DELIVERY METHOD ON EXTREMELY PREMATURE INFANTS VAGINAL DELIVERY OR CESAREAN SECTION

Ueda K. $^{1}$, Takahashi A. ${ }^{1}$, Watabe S. ${ }^{1}$, Waki K. ${ }^{1}$

Kurashiki Central Hospital, Pediatrics, Okayama-Japan ${ }^{1}$

\section{Introduction}

The optimal delivery method for extremely low birth weight infants (ELBWI) is controversial. In Japan, the proportion of caesarean section is different in institutions, from 10 $\%$ to $90 \%$. In our institution, cesarean section is often selected for ELBWI more than 23 weeks gestational age. But, we do not have a definite rule of delivery method. The aim of this study is to clarify the impact of delivery methods for ELBWI.

\section{Methods}

We conducted a retrospective study of singleton ELBWI less than 28 weeks gestational age and born at our hospital from 2005 to 2017. We excluded patients who were born at 22 weeks gestation or had complex malformations because of high mortality and morbidity, unfortunately. The primary outcomes examined were their mortality (discharge at death) and morbidity (respiratory distress syndrome, severe intraventricular hemorrhage, cystic periventricular leukomalacia, necrotizing enterocolitis, etc.) We measured developmental quotient (DQ) score with the Kyoto Scale of Psychological Development (KSPD) as secondary outcomes. The $\chi 2$ test and Mann-Whitney U test was used. Statistical significance was set at $\mathrm{p}<0.05$.

\section{Results}

There were 21 infants born by vaginal delivery and 18 (86\%) were survived (Group V), and there were 190 infants delivered through caesarean section and 170 (89\%) were survived (Group C). There were no significant differences for gestational age, birth weight, sex. Data of umbilical cord blood $\mathrm{pH}$ were significantly lower in group $\mathrm{V}$. There were more maternal fever and chorioamnionitis in group $\mathrm{V}$. There were no significant differences in the rates of mortality and morbidity. The median (IQR) of total DQ of group V and group C were 88 (7296) and 89 (78-97) at corrected 1.5 years. The median (IQR) of total DQ of group V and group C were 79 (62-85) and $83(71-94)$ at corrected 3 years. The neurodevelopmental outcomes were not different significantly between both groups.

\section{Conclusion}

Our results suggest that prematurity alone should not be a valid indication for cesarean section. Decision of delivery method is one of important issues but neurodevelopmental prognosis might be more affected by optimal extension of pregnancy period, good oportunity of delivery, waiting of expert medical team for a baby, proper management for a long time and so on. 
E1220 - THE IMPACT OF ANTENATAL CORTICOTHERAPY IN INFANTS BORN BEFORE 33 WEEKS'GESTATION A COMPARATIVE STUDY OF 565 PREMATURES

Ben Thabet A. ${ }^{1}$, Ben Hamad A. ${ }^{1}$, Abdelkarim R. ${ }^{1}$, Charfi M. ${ }^{1}$, Regaieg C. ${ }^{1}$, Hamida N. ${ }^{2}$, Regaieg R. ${ }^{1}$, Bouraoui A. ${ }^{1}$, Gargouri A. ${ }^{1}$

Hedi Chaker Hospital, Neonatology, Sfax-Tunisia ${ }^{1}$

Hedi Chaker Hospital, Neonatotogy, Sfax-Tunisia ${ }^{2}$

\section{Objective}

The aim of our study was to assess the impact of antenatal corticotherapy on hospital morbidity and mortality in premature infants delivered before 33 weeks' gestation.

\section{Methods}

This is a retrospective and comparative study of all premature infants born before 33 weeks'gestation at the maternity hospital and hospitalized in the neonatology department of Hedi Chaker university hospital in Sfax between 1January 2013 and 31 December 2015. We compared the short-term prognosis in premature infants, whether antenatal corticotherapy was used or not.

\section{Results}

During the study period, 565 premature infants born before 33 WG were hospitalized. The rate of very premature babies was $1.9 \%$.

$76.1 \%$ of them had received antenatal corticotherapy.

Antenatal corticosteroid therapy has reduced the frequency of hyaline membrane disease (22.8\% versus $44.4 \%$ ).

The incidence of intraventricular hemorrhage and necrotizing enterocolitis was higher in preterm infants who did not receive antenatal corticosteroid therapy.

The overall hospital mortality was $25.1 \%$ in this population. It was significantly higher in case of absence of antenatal corticosteroid therapy (39.3\% versus 20.7\%) The main causes of death were immaturity, severe hyaline membrane disease, healthcareassociated infections and apnea with intraventricular haemorrhage.

\section{Conclusion}

The efficiency of antenatal corticotherapy in these premature infants has been well demonstrated. It decreases neonatal morbidity and mortality.

Other areas of research should be explored, including the impact on growth, cognitive development and adult health. 
E1240 - THE NEURODEVELOPMENTAL OUTCOME AT THE FIRST YEAR OF LIFE OF ELBW AND VLBW NEONATES BY MODE OF DELIVERY

Moutafi A. ${ }^{1}$, Yatrakou E. ${ }^{1}$, Katechi K. ${ }^{1}$, Liouli C. ${ }^{1}$, Diamantopoulou M. ${ }^{1}$, Anatolitou F. ${ }^{1}$ Agia Sophia Childrens Hospital, A' Nicu, Athens-Greece ${ }^{1}$

\section{Introduction}

The optimal mode of delivery of VLBW infants remains controversial. However as in all deliveries caesarian section is becoming more prevalent. We aimed to explore the results of this trend in our own neonatal population.

\section{Objective}

The purpose of this study is to monitor neurodevelopment outcomes of ELBW and VLBW neonates by mode of delivery.

\section{Methods}

It is a retrospective study. We reviewed the hospital records of 139 neonates hospitalized during the years 2016-2018, from their follow up evaluation.

\section{Results}

57of this neonates had a birth weight $<1000$ gr and 82 had birth weight $<1500$ gr. We also investigated the mode of delivery, the ultrasound scan findings and the need for early intervention.

\section{Conclusion}

During this period we observed a gradual decrease of natural childbirths with simultaneous increase of the survival rate. The mortality rate was higher among the neonates which were born by natural childbirth. This group had increased frequency of pathological findings in the brain ultrasound. In addition they needed early intervention during the first year of life more frequently than the neonates which were born by caesarian section. Our data are consistent with similar studies however larger series are needed to evaluate the best mode of delivery of this vulnerable population 


\section{E1321 - ACHONDROPLASIA IN TWIN}

Balasar Ö. ${ }^{1}$, Balasar B. ${ }^{2}$, Doğru Ş. ${ }^{3}$, Örs R. ${ }^{4}$

T.C. Ministry Health Konya Education Research Hospital, Medical Genetics, Konya-Turkey ${ }^{1}$

T.C. Ministry Health Konya Hadim State Hospital, Clinical Microbiology and Infectious

Disease, Konya-Turkey ${ }^{2}$

T.C. Ministry Health Konya Beyhekim State Hospital, Obstetrics and Gynecology, KonyaTurkey $^{3}$

Konya Medova Hospital, Neonatology, Konya-Turkey ${ }^{4}$

\section{Objective}

Achondroplasia (OMIM \# 100800), the most common form of chondrodysplasia, is characterized by disproportionate short stature, rhizomelic shortness, lumbar lordosis, brachydactyly, macrocephaly, frontal prominence, flattened nasal root and brachydactyly. The estimated incidence is 1: 25000 live births. The disease has autosomal dominant inheritance and is the most common cause of disproportionate short stature. It is associated with advanced paternal age and occurs in $80 \%$ of cases as a result of a de novo mutation. It occurs as a result of mutation in fibroblast growth factor receptor3 (FGFR3) gene. The FGFR3 gene encodes a transmembrane receptor that is important in regulating linear bone growth.

\section{Case}

Newborn male twin patients were evaluated for rhizomelic shortness in our clinic. They were born from the third pregnancy of a 37-year-old father and 35-year-old mother with a 31-week cesarean section. Prenatal ultrasonographic examination showed shortening of rhizomelia. The birth weight of the patients was measured as $1870 \mathrm{gr}$ and $1400 \mathrm{gr}$ and the height at birth was $40 \mathrm{~cm}$. Physical examination revealed rhizomelic shortness, broad forehead, flattened nose root, hypertelorism, short thorax, small hand and foot, short and wide fingers, curvature of forearm and leg. Chromosome analysis and FGFR3 gene analysis from peripheral blood were requested with a preliminary diagnosis of achondroplasia.

\section{Conclusion}

Chromosome analysis of peripheral blood was performed as normal karyotype. A heterozygous mutation of c.1138G> A (p.Gly380Arg) was identified in the FGFR3 gene. Achondroplasia is a genetic disease that can be easily diagnosed during prenatal and neonatal periods. Molecular genetic testing is important to confirm clinical diagnosis and provide appropriate genetic counseling. 


\section{E1389 - BEING A REFUGEE CONSTITUTES RISK TO EARLY TERM DELIVERY}

Okman E. ${ }^{1}$, Beser Ozmen E. ${ }^{1}$, Kucukoglu Keser M. ${ }^{1}$, Alyamac Dizdar E. ${ }^{1}$, Sari F. ${ }^{1}$, Oguz S. ${ }^{1}$

University of Health Sciences, Zekai Tahir Burak Women’s Health, Health Application and Research Center, Neonatology, Ankara-Turkey ${ }^{1}$

\section{Objective}

The birth rate of Syrian refugees in Turkey arises. Therefore we aim to evaluate the birth characteristics of the infants born from Syrian refugee mothers and particularly emphasize the importance of perinatal care in refugees.

\section{Methods}

This study was conducted from October to December 2018. Medical records of Syrian and Turkish infants whose gestational age $\geq 37$ weeks were retrospectively evaluated. Study infants in both groups were classified based on gestational age, as early term (370/7 - 386/7 weeks), full term and late term (390/7 - 416/7 weeks).

\section{Results}

The study includes 225 infants of whom 96 were Syrian and 129 were Turkish. Statistical analysis indicate a significant difference in gestational weeks, where Syrians were lower than Turkish (38.2 \pm 1.1 vs $39.1 \pm 1.4$ weeks; $\mathrm{p}<0.001)$. It was observed that $70 \%$ of Syrian infants were early-terms, with compare to $57 \%$ of Turkish infants $(\mathrm{p}<0.001)$. Syrian mothers had gained less weight throughout their pregnancies $(10 \pm 4 \mathrm{~kg}$ vs $12 \pm 5 \mathrm{~kg} ; \mathrm{p}=0.001)$ Maternal age, weight and body mass index, mode of delivery, and birth weight of the infants were similar between groups.

\section{Conclusion}

Lower socio-economic condition of Syrian mothers or the stress they are exposed to, constitutes risk to an early-term delivery. Early term deliveries and related morbidity and mortality rates may be reduced if refugees have access to appropriate perinatal care. 


\section{E1395 - IMPACT OF MODE OF DELIVERY ON NEONATAL ADAPTATION} Subotic G. ${ }^{1}$, Nikolic T. ${ }^{2}$

General Hospital Cacak, Neonatology, Cacak-Serbia ${ }^{1}$ Clinical Center of Serbia, Clinic for Gynecology and Obstetrics, Beograd-Serbia ${ }^{2}$

\section{Objective}

The caesarean section has become the most commonly performed surgical procedure in developed countries, and its incidence is constantly increasing. Caesarean section, especially elective, carries the risk of iatrogenic prematurity, but also the risk of complications in newborns, due to lack of humoral factors and physiological mechanisms that are activated during spontaneous labor, so the objective of this study was to investigate the differences in adaptation of neonates according to mode of delivery.

\section{Methods}

This retrospective cross-section study, undertaken at maternity unit of General Hospital Čačak, included 150 newborns, 50 born by vaginal delivery (VD), 50 by cesarean section after trial of labor (CTL) and 50 by elective cesarean section (ECD) from uncomplicated in term singleton pregnancies. Commonly assessed neonatal outcome variables (Apgar at 1 and 5 minutes, required measures of resuscitation and the occurrence of transient tachypnea of the newborn - TTN) were used to compare condition at birth, using Kruskal-Wallis and Chisquare testing. Correlation between mode of delivery and neonatal outcome variables was assessed using Spirman's coefficient.

\section{Results}

In the VD group, gestational age was 37 - 40 weeks, with $12 \%$ of newborns born before 39 gestational weeks. In the CTL group, the range was 37 - 41 weeks, with $22 \%$ born before 39 weeks, while in the ECD group, gestational age was $37-41$ weeks, but $42 \%$ of newborns were born before 39 gestational weeks, $(\mathrm{p}<0.001)$. Apgar in 1 minute did not differ significantly. Apgar in 5 minutes was significantly different between the groups $(p=0.009)-$ CTL group had the lowest score, while the other two groups did not differ significantly. Delivery room resuscitation was required in 23.3\% cases, which included 6\% neonates in VD group, 44\% in CTL and 20\% in ECS group ( $\mathrm{p}<0.001)$. Routine procedures (drying and stimulation) were required in $16.7 \%$ and $11.3 \%$ cases respectively, while bag/mask ventilation was required in $16 \%$ cases. TTN was diagnosed in $24 \%$ of newborns $(8 \% \mathrm{VD}, 30 \% \mathrm{CTL}$ and $34 \%$ ECS; $\mathrm{p}=0.005)$. In VD, TTN was significantly less common than in CTL and ECS groups. The occurrence of TTN did not differ significantly between CTL and ECS. The mode of delivery had a significant correlation with the occurrence of TTN. Also, there was a higher likelihood of TTN in neonates of lower gestational age and in cases where TTN occurred, a higher respiratory rate was registered in neonates of lower gestational age. The association of observed variables was stronger for gestational age than for mode of delivery.

\section{Conclusion}

Method of delivery affects the adaptation of the newborn. Whenever possible, delivery should be done vaginally, and in cases of elective cesarean section, it is recommended not to be done before 39 weeks of gestation. 
E1444 - PERIVIABLE BIRTH RESULTS IN A TERTIARY HOSPITAL AND DECICION MAKING IN AN ETHICAL DILEMMA

Papathoma E. ${ }^{1}$, Gavrili S. ${ }^{1}$, Koutroumanis P. ${ }^{2}$, Kovlatzi A. ${ }^{1}$, Andreou C. ${ }^{1}$, Bagiati D. ${ }^{1}$, Varela P. ${ }^{1}$, Sevastiadou S. ${ }^{1}$

General Hospital of Athens "Alexandra”, Nicu, Athens-Greece ${ }^{1}$

General Hospital of Athens “Alexandra”, Department of Gynecology and Obstetrics, AthensGreece $^{2}$

\section{Objective}

Gestational age has been used as a guide to resuscitation decision-making in preterm infants because it is strongly associated with prognosis. Over the last 2 decades, advances in neonatal management and medical technology, led to the improvement of the survival rates of infants born at $<$ or $=28$ weeks of gestation; however, available data on survival rates and outcomes of babies born at the threshold of viability from 22 to $25+6$ weeks of gestation display wide variation by country and show that significant morbidity and disability still persists. The commitment and striving for their care has complex medical, social and ethical implications, while decision-making is a crucial issue that involves the infant, the family and the health care providers.

\section{Methods}

A retrospective analysis of collected data of infants born between $22+0$ and 25+6 weeks gestation admitted to our level III neonatal intensive care unit (“Alexandra” General Hospital, Athens) over the period of 3 years (2016-2018) regarding survival rates and causes of death.

\section{Results}

A total of 47 babies born between $22+0$ and $25+6$ weeks

Mortality rates were 46.6\%, 40\% and 35.7\% for the years 2016, 2017 and 2018 respectively. There were multiple causes of death, including immaturity, very low birth weight, respiratory distress syndrome, early onset neonatal infection, pneumothorax, necrotizing enterocolitis. Birth weight was an important factor for survival and for babies weighted 400-499 gr, 500599 gr, 600-699 gr and >= 700 gr the survival rates were $0 \%, 66.6 \%, 68.7 \%$ and $92.8 \%$ respectively.

\section{Conclusions}

Results show a significantly increased survival rate during the past two years, which is consistent with the continuous improvement in quality of maternal, delivery and newborn care provided in the obstetrics and neonatology departments of our hospital. Given to the characteristics of the maternal population referred to our hospital, such as absent or poor prenatal care, immigrants, women with serious pathology of pregnancy, as well as the recent change in legislation regarding the limit of viability at 22 weeks, we provide resuscitation to all babies above 22 weeks, followed by reassessment or intensive care, depending on each case. Apart from gestational age a number of factors such as birthweight, sex, the use of antenatal corticosteroids, singleton or a multiple gestation.

Providing resuscitation and care in periviable babies is a clinically and ethically complex issue. Decisions made by the clinicians, should ideally be based in medical and ethical reasons and should be consistent with parents wishes, without forgetting to act as the baby's advocate when needed. 


\section{E1476 - DEVELOPMENTAL OUTCOMES OF POSTOPERATIVE FORMER PRETERM INFANTS IN CORRECTED 6 AND 12 MONTH OF AGE}

Kandelaki E. 1, Kherkheulidze M. 1, Kavlashvili N. 1, Solomonia N. ${ }^{2}$ Tbilisi State Medical Universitty, Pediatrics, Tbilisi-Georgia ${ }^{1}$ Iashvili Childrens Central Hospital, Pediatrics, Tbilisi-Georgia ${ }^{2}$

\section{Objective}

To compare developmental outcomes of preterm infants with infants born at same gestational age but with history of surgery due to abdominal congenital anomalies at same corrected age of 6 and 12 month

\section{Methods}

Sample included total 46 former preterms born at 34-36 weeks of gestation. Study group was 22 preterms with history of previous surgery due to intestinal congenital problems and 24 late preterms with minimal problems in early neonatal period. Assessment of development was performed by Bayley III tool. We compared mean developmental scores in cognitive, motor and language areas in serial cross-sectional analyses at each time point using multi variant linear regression.

\section{Results}

With covariates controlled at all time points at 6 months preterm infants with surgery demonstrated less optimal developmental outcomes in two major areas - motor and language development, compared with preterm infants without surgery but born early $(\mathrm{P}<.0001)$. Difference was not seen in cognitive developmental area and outcome was similar for both groups. At 12 month corrected of age motor developmental scores were still significantly low in surgery group in comparison to preterms only. They demonstrate similar scores in cognitive and language areas of development.

\section{Conclusion}

Preterm infants with surgery intervention demonstrate less developmental outcomes in motor area at 12 months, they demonstrate similar outcomes in other areas of development. Results can indicate on more in depth assessment of development of preterms after surgical pathologies. 


\section{E1483 - DO ANTENATAL CORTICOSTEROIDS IMPROVE NEONATAL OUTCOMES OF LATE PRETERM INFANTS?}

Özge Aydemir ${ }^{1}$, Melih Velipasaoglu ${ }^{2}$, Damla Güneş ${ }^{1}, \underline{\text { Neslihan Tekin }}{ }^{1}$

Eskişehir Osmangazi University Faculty of Medicine Neonatology Eskişehir-Turkey ${ }^{1}$

Eskişehir Osmangazi University Faculty of Medicine Obstetrics and Gynecology EskişehirTurkey $^{2}$

\section{Objectives}

Eight percent of all deliveries occur in the late preterm period. Infants who are born late preterm have more neonatal complications than do newborns born at term. Administration of antenatal corticosteroids (ANCS) to women at risk for late preterm delivery was shown to reduce the rate of neonatal respiratory complications. We aimed to investigate the affects of ANCS on neonatal intensive care unit (NICU) admission rates and other short term neonatal outcomes of late preterm infants.

\section{Methods}

A retrospective cohort study was conducted. After October 2017 ANCS were recommended to all women at risk for late preterm delivery in our hospital. Infants born late preterm (34 weeks 0 days to 36 weeks 6 days) between October 2017 and September 2018 whose mothers received 2 intra-muscular injections of $12 \mathrm{mg}$ betamethasone were included as the study group. Infants born late preterm between October 2016 and September 2017 whose mothers did not receive any ANCS were taken as the control group. Infants with maternal chorioamnionitis, congenital anomalies and incomplete course of ANCS were excluded. Two groups were compared in terms on NICU admissions, rate and severity of respiratory complications and other common problems encountered in the early neonatal period. Results: Infants in the study group $(n=84)$ and the control group $(n=103)$ were similar in terms of gestational age, birth weight, type of delivery and antenatal characteristics. Males were higher in number in the control group (\%56 vs \%38, $\mathrm{p}=0.013$ ). Rates of NICU admissions, respiratory complications, mechanical ventilation requirement, sepsis, feeding problems, necrotizing enterocolitis, jaundice, and duration of hospitalization were similar between groups $(\mathrm{p}<0.05)$. Hypoglycaemia was more common in the study group $(10.71 \%$ vs $2.91 \%$, $\mathrm{p}=0.027)$.

\section{Conclusion}

Administration of ANCS to women at risk for late preterm delivery significantly increased the risk of hypoglycaemia with no improvement in neonatal outcomes. At early gestational ages it is clear that ANCS decrease neonatal mortality and morbidity and improve long-term outcomes. However, in the late preterm period these benefits are less clear. Furthermore, effects of hypoglycaemia on neurodevelopment in conjunction with ANCS exposure in late preterm born infants are unknown. We believe that caution should be exercised before routine use of ANCS for women at risk for late preterm delivery, before long-term effects are well described. Further studies should focus on long-term outcomes of ANCS as well as defining which subgroups among late preterm infants could benefit ANCS. 


\section{Neonatology - Necrotising Enterocolitis}

\section{E1398 - RECTAL LEVOTHYROXINE FOR THE TREATMENT OF NEONATAL HYPOTHYROIDISM AN ALTERNATIVE ROUTE OF ADMINISTRATION}

Aygun E. ${ }^{1}$, Ince Z. $^{1}$, Coban A. ${ }^{1}$

Department of Pediatrics, Division of Neonatology, Istanbul University, Istanbul Faculty of

Medicine, Istanbul-Turkey ${ }^{1}$

Hypothyroxinemia in preterm infants is a transient thyroid dysfunction with normal TSH but low fT4 levels. In cases where enteral drug administration is not possible an alternative route of therapy is required. The treatment of congenital hypothyroidism in a preterm newborn with short bowel syndrome with rectal diluted levothyroxine is presented. A male baby was born at 256/7 weeks of gestation with a birthweight of $665 \mathrm{~g}$ by caesarean section because of severe preeclampsia. He was intubated, transferred to the NICU on mechanical ventilation. Minimal enteral nutrition and TPN were started on the first day of life. Necrotizing enterocolitis (NEC) was diagnosed clinically and radiologically in the infant who did not pass meconium until the 4th postnatal day. Enteral feeding was stopped, nasogastric drainage and broad-spectrum antibiotic therapy were started. The baby needed surgical intervention for intestinal perforation on the 6th day of life. A long bowel segment including the jejunum and ileum was resected because of multiple perforation areas and diffuse circulatory disturbance of the intestinal wall. Only a very short bowel segment could be reserved and an enterostomy was made. TPN feeding was continued. On the 8th postnatal day, cholestatic jaundice developed and direct bilirubin levels gradually increased with STB level reaching $18.9 \mathrm{mg} / \mathrm{dL}$, DB 14.4 $\mathrm{mg} / \mathrm{dL}$ on postoperative 21st day. Cholestasis could be associated with sepsis, prolonged TPN and hypothyroxinemia. Thyroid function tests were in the low normal region on the 14th postnatal day. Control levels on the 21 st day showed hypothyroxinemia and enteral levothyroxine treatment was started (Table). There was no response to this therapy which was thought to be the result of insufficient absorption of the drug due to short bowel syndrome. As parenteral and suppository forms are not available in our country it was decided to administer the medication rectally with diluted levothyroxine tablets. Thyroid function tests reached normal levels and cholestasis resolved after this therapy. A few cases of rectal diluted L-T4 treatment with successful results has been reported in infants diagnosed with short bowel syndrome similar to our case. As there is not enough data about the rectal absorption of the enteral form of this drug, it was decided to start with a dose of $10 \mathrm{mcg} / \mathrm{kg} /$ day which is the recommended starting enteral dose. In conclusion timely treatment of hypothyroidism is important for normal growth and brain development. Rectal levothyroxine should be considered as an alternative, safe and effective route for the treatment of hypothyroidism when other routes are unavailable.

Table 1: Levothyroxine treatment and thyroid function tests

PN age (days) 14212835425060

fT4 (pmol/L) 11,20 8,45 7,20 8,50 12,30 17,50 16,00

TSH (mIU/L) 0,61 0,19 0,18 1,90 5,50 0,06 3,70

Cortisol, $\mu \mathrm{g} / \mathrm{dL} 5,750,510,96$

L-thyroxine route (dosage) Enteral $(5 \mu \mathrm{g} / \mathrm{kg})$ Enteral $(10 \mu \mathrm{g} / \mathrm{kg})$ Rectal $(10 \mu \mathrm{g} / \mathrm{kg})$ Rectal $(10 \mu \mathrm{g} / \mathrm{kg})$ Rectal $(10 \mu \mathrm{g} / \mathrm{kg})$ Rectal $(10 \mu \mathrm{g} / \mathrm{kg})$ 


\title{
Neonatology - Lung injury and long-term lung function
}

\author{
E1117 - A SURVEY OF OBSTETRICIANS AND NEONATOLOGISTS OPINION ON \\ CORTICOSTEROID ADMINISTRATION FOR FETAL LUNG MATURATION \\ Hassan R. ${ }^{1}$, Lindow S. ${ }^{1}$ \\ Sidra Medicine, Obstetrics, Doha-Qatar ${ }^{1}$
}

\section{Objective}

The aim of the survey was to gain an understanding of obstetricians and neonatologist opinions on the administration of corticosteroid for fetal lung maturation due to observed variation in decision making. The results will aid in the development of guidelines for our unit in areas where there is insufficient evidence in literature.

\section{Methods}

A questionnaire survey was sent out electronically to all consultant neonatologists and obstetricians working within a tertiary obstetric unit in Qatar. Data collected included years of clinical experience, gestation at which to administer steroids for planned elective caesarean delivery or preterm labor, type and regime of corticosteroid used, frequency of repeat doses of corticosteroid.

\section{Results}

A total of 68 consultants responded. 53 of the respondents were obstetricians and 15 neonatologists. Over half of the respondents $56 \%(n=32)$ stated they would not administer antenatal corticosteroid for women undergoing an elective caesarean section between 38+0 and $38+6$ weeks. $65 \%(n=32)$ would administer corticosteroids for women undergoing planned elective caesarean at less than 38 weeks. $27 \%(n=15)$ would consider a repeat dose of corticosteroids if the prior course was administered more than 14 days before. $67 \%(n=37)$ would consider a repeat dose if the prior dose was given between 24 and 25+6 weeks of gestations. $77 \%(\mathrm{n}=44)$ stated they would administer betamethasone $12 \mathrm{mg}$ intramuscularly 24hrs apart. There was no significant difference in opinions between the neonatologists and obstetricians.

\section{Conclusion}

This survey indicates that there is varying opinions on the administration of corticosteroids particularly for the gestation for planned elective caesarean section. Over half would not consider corticosteroids over 38 weeks of gestation. It highlights the importance for healthcare professionals to understand the evidence for the optimal timing and benefit of antenatal corticosteroid for fetal lung maturation. Administration of corticosteroid at certain gestations has been proven to be of significant benefit. However there is also emerging evidence of possible long term effects of corticosteroids on child development, therefore it is important to determine the optimal gestations where benefit outweighs the risks and avoid repeat doses. This survey will allow us to take into consideration the opinions of consultants where there is insufficient evidence to guide management. 


\section{E1383 - A RARE CAUSE OF RESPIRATORY DISTRESS IN NEWBORN PNEUMOMEDIASTINUM}

Gülcan Kersin S. ${ }^{1}$, Özdemir H. ${ }^{1}$, Bilgen . $^{1}{ }^{\text {, Ülgen Ö. }}{ }^{2}$, Memişoğlu A. ${ }^{1}$, Özek E. ${ }^{1}$

Marmara University, School of Medicine, Department of Pediatrics, Division of

Neonatology, Istanbul-Turkey ${ }^{1}$

Marmara University, School of Medicine, Department of Pediatrics, Istanbul-Turkey ${ }^{2}$

\section{Introduction}

Pneumomediastinum is an uncommon cause of neonatal respiratory distress and occurs in approximately 2.5 per 1000 live births. Although the most common cause of pneumediastinum is an underlying lung disease, it can be seen in newborns who underwent resuscitation during delivery without a predisposing factor. In this case report, we aimed to present a patient who underwent resuscitation in the delivery room and developed pneumomediastinum.

\section{Case}

A female baby was born at 37 weeks of gestation with a birth weight of $3675 \mathrm{~g}$ to a 37 year old multiparous mother by vaginal delivery. She required positive pressure ventilation by Tpiece after delivery. The baby's 1st and 5th minute APGAR scores were 7 and 9, respectively. Due to grunting and tachypnea the baby was intubated and was admitted to the neonatal intensive care unit. The physical examination of the baby revealed edematous eyelids, petechiae and ecchymosis around the eyes. Breath and heart sounds were decreased on the right hemithorax. Crepitation was palpated bilaterally on the neck and upper chest wall due to subcutaneous emphysema. Pneumomediastinum was suspected due to sail sign detected on the chest X-ray. Computed Tomography scan was performed and did not show any mediastinal mass and it was related with birth trauma in our case. The baby was extubated after 24 hours. Pneumomediastinum spontaneously regressed during clinical follow-up and the baby was discharged on the 7th day of life.

\section{Conclusion}

Pneumomediastinum is a rare cause of respiratory distress and initial diagnosis by chest X-ray can be difficult in some cases. Pneumomediastinum should be considered especially in patients with respiratory distress who underwent resuscitation after a traumatic delivery. The "sail sign" is an uncommon radiological appearance of pneumomediastinum. Careful conservative management can result with spontaneous resolution without longterm sequelae. 


\title{
Neonatology - Neonatal Nutrition
}

\author{
E1136 - ANTENATAL EDUCATION FOR CHILDBIRTH AND PARENTING \\ PREPARATION DURING PREGNANCY IN GREECE \\ Daglas $M .^{1}$, Antoniou E. ${ }^{1}$, Pechlivani F. ${ }^{1}$ \\ University of West Attica, Midwifery, Athens-Greece ${ }^{1}$
}

\section{Objective:}

Pregnancy, labour and puerperium are especially sensitive periods for women. The preparation of pregnant women and their partners during pregnancy (antenatal classes) for childbirth and parental role is provided by midwives, who meet the emotional and psychological needs of the couples. This specific antenatal education and parenting preparation is a necessity for woman and her partner. The aim of this research is to study the preparation degree of the couples for childbirth and parenthood during antenatal period in Greece and to what degree this antenatal preparation is related to public health issues, such as feelings and women's emotional experience during pregnancy, type of birth and breastfeeding.

\section{Methods}

We studied the opinions of 244 mothers (giving birth in the last 2 years). Outcome measures were assessed through self-administered questionnaire. Demographic and obstetric data were collected from participants.

\section{Results}

Although the majority of women mention positive feelings during their pregnancy, a big percentage (80.5\%) say that they were anxious about the progress of the pregnancy; furthermore, $72.3 \%$ say that they were concerned about their maternal role, $74.5 \%$ were afraid for the embryo's health during pregnancy and $69.9 \%$ for the labour process. The percentage of women that have attended antenatal classes and parenting preparation programmes during pregnancy is $34.8 \%$. All of them say that the programmes were interesting and helped them to a big extent to have positive feelings for pregnancy, labour and puerperium. The participants in these programmes: a) had normal births to a bigger degree than the ones that did not participate $(\mathrm{Pv}=0), \mathrm{b})$ exclusively breastfed $(\mathrm{Pv}=0.015)$ for a period bigger than 6 months $(\mathrm{Pv}=0)$ and $\mathrm{c})$ chose caesarian section to a smaller percentage $(\mathrm{Pv}=0)$.

\section{Conclusion}

Antenatal education for childbirth and antenatal parenting preparation programmes may have a beneficial main effect on normal birth percentage and breastfeeding rate. There is a need for greater effort to increase antenatal education and parenting preparation awareness during pregnancy in Greece. 
E1442 - HUMAN MILK AND PRETERM NEONATES

Papathoma E. ${ }^{1}$, Giannakos C. ${ }^{2}$, Stratiki E. ${ }^{1}$, Leontari K. ${ }^{1}$, Sourgiadaki I. ${ }^{1}$, Bakirtzi N. ${ }^{1}$ Diamandi A. ${ }^{1}$, Gavrili S. ${ }^{1}$

General Hospital of Athens "Alexandra”, Nicu, Athens-Greece ${ }^{1}$

National Technical University of Athens, School of Electrical and Computer Engineering, Athens-Greece ${ }^{2}$

\section{Objective}

Human milk is thought to be the optimal nutrition for term and preterm infants during the first 6 months of life. While the nutrient composition of human milk is ideal for term infants, it is insufficient to meet the needs of the growing preterm infant. Under that consideration, human milk is fortified with BMF-Breast Milk Fortifiers so it can help achieve a satisfactory growth for preterm infants. The purpose of the study is to compare body weight and head circumference between preterm infants who were fed with human milk and those who were fed with formula milk.

\section{Methods}

A total of 800 neonates were studied, of which 250 were fed with modified formula milk for preterm infants, 300 with human milk and 350 with human milk fortified with BMF. Statistical analysis with t-test was used.

\section{Results}

Infants were reviewed at 6 months of age (chronological age) and it was found that the body weight of those who were fed by formula milk was above 95th percentile, regardless of prematurity. Those who were fed by human milk had a body weight that was at 45th- 50th percentile and those who were fed by human milk fortified with BMF had a weight between 55-75th percentile. Regarding the head circumference, it was in 75-85th percentile for those fed by formula milk, in 50-75th percentile for those fed by human milk and in 50th percentile for those fed by human milk fortified with BMF.

\section{Conclusion:}

Evidence indicates that human milk is the best nutrient uniquely suited not only to term but also to preterm infants and human milk fortified with BMF was proven to be ideal according to the growth charts at 6 months. Feeding with formula milk was associated with increased risk for metabolic syndrome in adulthood as body weight was above 95th percentile at 6 months. In the future, research needs to address the nutritional management in specific groups of preterm infants such as IUGR infants and the quality improvement of fortifiers. 


\section{E1443 - HUMAN MILK AND IUGR NEONATES}

Papathoma E. ${ }^{1}$, Giannakos C. ${ }^{2}$, Stratiki E. ${ }^{1}$, Bogiatzis K. ${ }^{1}$, Leontari K. ${ }^{1}$, Sourgiadaki I. ${ }^{1}$, Zantiotou M. ${ }^{1}$, Sevastiadou S. ${ }^{1}$, Gavrili S. ${ }^{1}$

General Hospital of Athens "Alexandra”, Nicu, Athens-Greece ${ }^{1}$

National Technical University Of Athens, School of Electrical and Computer Engineering, Athens-Greece ${ }^{2}$

\section{Objective}

Human milk is the best source of nutrition for both term and preterm infants during the first 6 months of life, granting health benefits both in the short and long-term. For preterm infants, human milk is recommended to be fortified with BMF-Breast Milk Fortifiers so as to help to achieve a satisfactory growth in premature infants. Neonates with intrauterine growth retardation (IUGR) are a special group of infants with an increased risk of milk intolerance and necrotizing enterocolitis. There are no clear guidelines regarding the optimal feeding of these infants and feeding practice varies between the various Neonatal Intensive Care Units. The purpose of this study is to compare the body weight of term and preterm IUGR infants who were fed with human milk, human milk fortified with BMF and formula milk at 6 months of life (chronological age).

\section{Methods}

A total of 950 IUGR neonates were studied, of which 300 were premature (between 30 and 35 weeks of gestation) and 650 were term. Regarding the premature IUGR infants, 80 were fed with human milk, 86 with human milk fortified with BMF and 94 with formula milk. Statistical analysis with t-test was used.

\section{Results}

Regarding the term infants, those who were fed with human milk, at 6 months of age had a weight that was between 50-75th percentile at growth charts, while those that were fed with modified formula milk had a weight above the 95th percentile. Regarding the premature IUGR infants, those fed with formula milk at 6 months of life had a weight that was at 95th percentile, while those fed with human milk had a weight between 50-75th percentile.

\section{Conclusions}

The term IUGR neonates who were fed with formula milk had a weight above the 95th percentile as well as an increased risk of developing metabolic syndrome in adulthood. On the contrary, postnatal catch up growth at 2 months of age occurred for those that were fed with human milk. The preterm IUGR neonates that were fed with formula milk had also increased risk of developing metabolic syndrome in adulthood. The preterm IUGR infants fed with human milk fortified with BMF, at 6 months of age had a weight that was between 50-75th percentile, a fact which signifies the impact of feeding with human milk fortified with BMF in infant's weight. 


\section{Neonatology - Hypoxic ischemic encephalopathy}

\section{E1025 - EARLY DETECTION OF HYPOXIC ISCHAEMIC ENCEPHALOPATHY BY METABOLIC CHANGES IN UMBILICAL CORD BLOOD}

Selimovic A. ${ }^{1}$, Skokic F. ${ }^{2}$, Dedic N. ${ }^{3}$

Clinic for Children Diseases, University Clinical Center, Intensive Care Unit, Tuzla-Bosnia and Herzegovina ${ }^{1}$

Clinic for Children Diseases, University Clinical Center, Assistant Director for Medical Devices, Tuzla-Bosnia and Herzegovina ${ }^{2}$

Clinic for Gynaecology and Obstetrics, Department for Neonatology, Tuzla-Bosnia and Herzegovina $^{3}$

Perinatal asphyxia is a major cause of acute pediatric mortality and long-term neurologic sequelae among survivors.

\section{Objective}

To evaluate metabolic changes in umbilical cord blood in asphyxiated newborns and to correlate them to the brain damage

\section{Methods}

Seventy live-born term newborns from singleton pregnancies with perinatal asphyxia (investigated group) and 40 healthy term newborns (control group) were included to the investigation. Blood samples for metabolic parameters were collected from the umbilical vein.

\section{Results}

Brain damage occurred in 44/70 (62,9\%) asphyxiated newborns having hypoxic-ischemic encephalopathy (HIE): 25 (56,8\%) had Grade I; 12 (27,3\%) Grade II; and 7 (15,9\%) had Grade III HIE. Investigated group had significantly higher levels of magnesium and potassium, while levels of sodium, calcium and glucose were lower compared to the controls. Metabolic acidosis was predominant in investigated group. Positive correlation was found between magnesium ( $r h o=0,708 ; p<0,0001$ ), potassium level ( $r h o=0,819 ; p<0,0001$ ), and the grade of HIE, while calcium ( $r h o=0,820 ; p<0,0001)$, glucose $(r h o=0,819 ; p<0,0001)$ and sodium levels (rho=0,783; $<0,0001$ ) were in negative correlation with the severity of HIE. In multivariate regression model the best predictors for severity of HIE were low $\mathrm{pH}$ and hypoglycemia.

\section{Conclusion}

Perinatal asphyxia might produce metabolic changes in umbilical cord blood which express significant correlation with the severity of HIE. 


\section{E1150 - ASSOCIATION BETWEEN LOW APGAR SCORE AND MATERNAL LABOR AND FETAL VARIABLES PRELIMINARY RESULTS}

Machado Junior L. ${ }^{1}$, Sangalan Gerencer C. ${ }^{2}$, Nazareth Pinto C. ${ }^{2}$, Giacometti Prol E. ${ }^{2}$, Kon

R. ${ }^{3}$, Barbosa de Carvalho H. ${ }^{4}$

University of São Paulo, Centro De Saude Escola Butantâ, São Paulo-Brazil ${ }^{1}$

Hospital Municipal Universitário De São Bernardo Do Campo, Gynecology and Obstetrics, São Bernardo Do Campo-Brazil ${ }^{2}$

University of São Paulo, Centro De Saude Escola Butantã, São Paulo-Brazil ${ }^{3}$

University of São Paulo, Preventive Medicine, São Paulo-Brazil ${ }^{4}$

\section{Objective}

Apgar score, despite being an ancient tool, is still widely utilized. The low scores, especially in the fifth minute, are associated with higher short time neonatal morbidity and mortality, and also with long term consequences, even in the absence of other serious neonatal complications. The purpose of our study is to access what maternal/ fetal aspects are associated with low Apgar scores in the fifth minute in term pregnancies.

\section{Methods}

We are conducting a case-control study with term live births assisted in a public teaching hospital in São Bernardo do Campo, Brazil. We named cases the births of neonates with Apgar score of six or less in the fifth minute, and controls the first and second birth of neonates after the cases, with Apgar of seven or more in the fifth minute. Were excluded (for both cases and controls): less than 37 weeks pregnancies, breech presentation, multiple pregnancies. Were assessed maternal, fetal and labor variables.

\section{Results:}

We collected data of 17 cases and 22 controls. We already have some significant results, because the case-control strategy allows us to have them even in the beginning of data collection. Among 17 cases, 16 had at least one risk factor that is available before labor for low Apgar score (maternal disease, absence of prenatal care, fetal growth restriction, post dates pregnancy, advanced maternal age, oligohydramnios), compared with 11 among 22 controls, $\mathrm{p}=0.003$. In 16 cases submitted to cardiotocography, 9 had abnormal findings, compared to only one among 19 controls, $\mathrm{p}=0.001$. With respect to the variables that are available throughout labor, we had an average of 440.9 minutes between rupture of membranes and the birth for the cases and of 195.2 minutes for the controls. Despite not significant yet $(p=0.08)$, it seems to be a strong tendency, and we believe the difference will become significant as the sample becomes larger.

\section{Conclusion}

Despite not finished yet, we found some significant associations. One interesting finding is the likely association between time of ruptured membranes and the outcome. Traditionally, longer periods of ruptured membranes were considered risk factor for chorioamnionitis/fetal infection, but not for low Apgar scores. However, a recent study showed a strong association between this variable and hypoxic/ischemic encephalopathy. We expect to have at least 50 cases and 100 controls. We intend to utilize the variables available before labor and in early labor to build a risk score for low Apgar in the fifth minute. 
E1359 - NEURODEVELOPMENTAL OUTCOME OF SEVERE ACIDEMIC TERM INFANTS

Song E. ${ }^{1}$

Chonnam National University Medical School, Department of Pediatrics, Gwangju-Korea, South ${ }^{1}$

\section{Objective}

This study aimed to evaluate the perinatal factors associated with neonatal mortality and neurologic morbidity in term infants with severe acidosis.

\section{Methods}

We retrospectively reviewed the data of term infants with severe acidosis from January 2012 to December 2016. Severe acidosis was defined as initial $\mathrm{pH}$ of less than $<7.0$ and $\mathrm{BE} \geq-12$ $\mathrm{mmol} / \mathrm{L}$. We searched the clinical characteristics, morbidities of mother and infants, and neuroimaging results. Also infants were divided by neurodevelopmental impairment and evaluated the relating perinatal factors.

\section{Results}

Twenty three term infants had severe acidosis. Meconium stained amniotic fluid was found in 43.5\% and neonatal resuscitation with positive pressure ventilation and endotracheal intubation was needed in $43.5 \%$ and 26.1\%. Among 8 infants with HIE, stage 2 or higher were $87.5 \%$. Four infants (17.4\%) showed abnormal brain imaging taken before discharge. On the analysis, the longer duration of invasive mechanical ventilation $(\mathrm{P}=0.049)$ and increased need for gavage feeding $(\mathrm{P}=0.021)$ were found in neurodevelopment impairment group.

\section{Conclusion}

The presence of HIE and abnormal findings of brain imaging before discharge were found to be associated with abnormal neurodevelopmental outcome in term infants with severe acidosis. Even if the brain imaging test was normal, we should consider the long-term followup of severe acidemic term infants. 


\section{Neonatology - Neonatal Brain Injury and Neuroprotection}

\section{E1106 - THE EFFECT OF PERINATAL HYPOXIA ON ELECTROLITES IN TERM NEWBORNS}

Jovandaric M. ${ }^{1}$

Clinic For Gynecology and Obstetrics, Department of Neonatology, Clinical Center of

Serbia, Belgrade, Serbia, Neonatology, Belgrade-Serbia ${ }^{1}$

\section{Introduction}

Under hypoxia conditions, intracellular accumulation of calcium ions occurs as well as disturbed balance of other ions $\left(\mathrm{K}+, \mathrm{Na}+\right.$ and $\left.\mathrm{Cl}^{-}\right)$. Hypoxia prevents the flow of potassium through the cell membrane. Sodium accumulation in the cell during hypoxia is the immediate cause of intracellular accumulation of water, and chlorine ions passively follow sodium ions.

\section{Objective}

To the influence of perinatal hypoxia on electrolytes in term newborns

\section{Methods}

The study included 50 term newborns with hypoxia, delivered naturally or by caesarean section who were exposed to oxygen therapy after birth due to the development of respiratory distress syndrome with Apgar score (AS) of less than 7 (range 1-10) and $\mathrm{pH}$ less from 7.20. The control group consisted of 50 healthy term newborn babies, delivered naturally or by caesarean section with no need for oxygen therapy. In the first and second hours of life in both groups of neonates $\mathrm{pH}, \mathrm{pCO} 2, \mathrm{pO} 2, \mathrm{HCO} 3, \mathrm{BE}, \mathrm{Na}+, \mathrm{K}+$, ionized $\mathrm{Ca}++$ were determined from arterialized capillary blood. On the second day of life, $\mathrm{Na}+, \mathrm{K}+$ total $\mathrm{Ca}++$, Cl- , they were determined from venous blood in both groups.

\section{Results}

Comparison of pH, pCO2, pO2, $\mathrm{HCO} 3$ and $\mathrm{BE}$ in the first hour of life in the newborn with hypoxia showed lower $\mathrm{pH}$, higher $\mathrm{pCO} 2$, lower $\mathrm{pO} 2$, lower $\mathrm{HCO}$, and higher $\mathrm{BE}(\mathrm{p}<0.01)$. In the second hour of life in newborns with hypoxia, lower $\mathrm{pH}$, higher $\mathrm{pCO} 2$, lower $\mathrm{pO} 2$, and higher BE $(p<0.01)$, as well as lower HCO3 values were obtained without statistically significant difference $(\mathrm{p}>0.05)$.

By comparing $\mathrm{Na}+$ concentration in the first hour of life from arterialized capillary blood in the hypoxic newborn, lower values of $(\mathrm{p}<0.01)$ were obtained, lower concentrations of $\mathrm{Na}+$ were found in the second hour of life, but without statistically significant difference $(p>0.05)$. By comparing the $\mathrm{K}+$ concentration in the first hour and the second day of life, a higher $(\mathrm{p}<0.01)$ was obtained, in the second hour of life a higher $\mathrm{K}+$ value was found, but without statistically significant difference $(\mathrm{p}>0.05)$. By comparing the concentration of ionized $\mathrm{Ca}++$ in the first and second hours after birth (arterialized capillary blood), as well as $\mathrm{Na}+$, total $\mathrm{Ca}++, \mathrm{Cl}-$ plasma levels on the second day of life, lower concentrations $(\mathrm{p}<0.01)$ were obtained.

\section{Conclusion}

A statistically significant difference in the concentration of electrolytes in neonates with hypoxia opens the possibility of using new markers of perinatal asphyxia. 
E1152 - PROSPECTIVE SURVEILLANCE STUDY OF SEVERE HYPERBILIRUBINAEMIA IN THE NEWBORN RISK FACTORS FOR NEUROTOXICITY

Benrabah O. ${ }^{1}$, Ait Mouheb T. ${ }^{2}$, Arrada Z. ${ }^{1}$

Hospital Parnet, Medicine, Hussein Dey-Algeria ${ }^{1}$ Hospital Belfort, Medicine, El Harrach Algeria $^{2}$

Jaundice in the neonatal period is an extremely common condition. This condition is usually benign and resolved spontaneously. However, in case of intense jaundice, the newborn may be exposed to dangerous neurological complications responsible for permanent damage, due to the neurotoxicity of free bilirubin.

\section{Objective}

To identify the risk factors for the occurrence of neurosensory complications of severe hyperbilirubinemia. Summary

\section{Methods}

This is a cohort, analytic, and single-center prospective study conducted between January 2014 and December 2016 in newborns with severe neonatal jaundice with a BST> 200 mg / l hospitalized at neonatal units.133 newborns were collected during this period. A multivariate logistic regression analysis to determine the factors involved in the development of neurosensory sequelae.

\section{Results}

Severe jaundice accounted for $7.8 \%$ of all neonatal jaundice. The mean age was 3 days, and the neurological signs at admission were present in $18.8 \%$ of newborns. Favorable clinical and biological evolution has been the rule in the majority of cases. From a neurological and sensory sequelae point of view, 8 children had abnormal psychomotor development ranging from a simple psychomotor delay to severe overall developmental delay and 5 children had pathological PEA. The risk factors for development of neurosensory sequelae identified by logistic regression were: male sex (OR: 2 CI: 95\% (0.6-0.9)) ( $p=0.04)$, gestational age less than 38 SA (OR: 2.4 CI: 95\% (0.9-7.8) $(\mathrm{p}=0.02)$, the delay of management beyond 24 hours (OR: 2.32 CI: 95\% (1.99-7.42) $(\mathrm{p}=0.042)$, the presence of neurological signs in the acute phase (OR: $3.06 \mathrm{CI}$ : 95\% $(1.26-13)(\mathrm{p}=0.01)$ and a BST level greater than $300 \mathrm{mg} / \mathrm{l}$ (OR: $2.7 \mathrm{CI}$ : 95\% (1.34-9.53) $(\mathrm{p}=0.008)$.

\section{Conclusion}

The evolution towards the more and more early exits of maternity leads to a resurgence of neurological risk. This neurological risk prompts us to pay more attention to this pathology. Thus, we can only insist on a rigorous evaluation of risk factors, an early detection of newborns at risk of developing severe jaundice. 
E1293 - A SURVEY OF OBSTETRICIANS AND NEONATOLOGISTS OPINION ON THE GESTATIONAL AGE FOR ADMINISTRATION OF MAGNESIUM SULPHATE FOR FETAL NEUROPROTECTION

Hassan R. ${ }^{1}$, Lindow S. ${ }^{1}$

Sidra Medicine, Obstetrics, Doha-Qatar ${ }^{1}$

\section{Objective}

Magnesium sulphate given to women shortly before delivery has been shown to reduce the risk of cerebral palsy and protects gross motor function in those infants born preterm. The evidence on the use of magnesium sulfate at $<30$ weeks of gestation as opposed to $<34$ weeks is not clear. Although statistical significance was more likely to be demonstrated for outcomes in the trials recruiting women up to 34 weeks of gestation, this was partly due to increased sample size and statistical power. Most guidelines recommend using up to 32 weeks but there is no international consensus.

The aim of the survey was to gain an understanding of our obstetricians and neonatologist opinions on the gestational age of administration of magnesium sulphate for neuroprotection to aid us in the development of local guidelines for our unit.

\section{Methods}

A questionnaire survey was sent out electronically to all consultant neonatologists and obstetricians working within a tertiary unit in Qatar. Data collected included years of experience and gestation at which to administer magnesium sulphate for fetal neuroprotection.

\section{Results}

A total of 68 consultants responded. Of the respondents 53 were obstetricians and 15 neonatologists. The majority of the respondents $75 \%(n=51)$ stated they would administer magnesium sulphate to women who present in preterm labor between 24 and 30 weeks of gestation, $8.8 \%(n=6)$ between 24 and 32 weeks and $8.8 \%(n=6)$ between 24 and 34 weeks. There was no significant difference in opinions between the neonatologists and obstetricians.

\section{Conclusion}

This survey indicates that there is varying opinions on the upper gestational age for the administration of magnesium sulphate for neuroprotection. The majority would administer magnesium sulphate between 24 and 30 weeks of gestation. There is a need for further research into this area in order to develop international consensual recommendations. 


\section{E1400 - THROMBOSIS OF TORCULAR HEROPHILI IN A NEWBORN}

Aygun E. ${ }^{1}$, Coban A. ${ }^{1}$, Aslan M. ${ }^{1}$, Tuncel D. ${ }^{1}$, Bayramoglu Z. ${ }^{2}$, Ince Z. ${ }^{1}$

Istanbul University, Istanbul Faculty of Medicine, Department of Pediatrics, Division of Neonatology, Istanbul-Turkey ${ }^{1}$

Istanbul University, Istanbul Faculty of Medicine, Department of Radiology, Division of Pediatric Radiology, Istanbul-Turkey ${ }^{2}$

A G1P0 28-year-old mother delivered vaginally a baby boy at 40+6/7 weeks of gestational age, birth weight of $3300 \mathrm{~g}$ and head circumference of $35.5 \mathrm{~cm}$. The Apgar scores at 1 and 5 minutes were respectively 5 and 9 . He had no postnatal adaptation problems and the physical examination was normal. It was reported that antenatal ultrasound at the 22nd week of gestation showed an anechoic cystic mass measuring 46x34 mm in the posterior fossa of the fetal brain with irregular hyperechogenic areas and flow towards the cystic mass at the level of the confluence sinus. The preliminary diagnosis was "thrombosis of torcular herophili". Antenatal magnetic resonance imaging (MRI) at 28th week of gestation showed a dilated confluence sinus, thrombosis and expansion of superficial collateral vessels, changes due to slow blood flow whereas the deep venous system was patent. On the first postnatal day brain MRI and MR venography was performed which revealed thrombosis in the left transverse and confluence sinuses, foci of bleeding in the Sylvian fissure, old thrombi within the torcular sinus and a normal brain parenchyma (Figure 1 and 2). These findings confirmed the antenatal diagnosis of thrombosis of torcular herophili. The etiologic investigation of thrombosis was done and the results were as follows: Protein C, protein S, antithrombin, homocysteine levels were normal; antiphospholipid and anticardiolipin antibodies, Factor V Leiden, Prothrombin 20210 mutation were negative. Antithrombolytic therapy was not given. At the first postnatal week, a control brain MRI was performed which showed regression of the thrombosis of torcular herophili with old foci of bleeding in the Sylvian fissure. The neurological examination was normal. The patient was discharged on the 20th postnatal day. Neuromotor development of the baby was normal at 5 months of age. The follow-up continues without any treatment. In conclusion thrombosis of torcular herophili is a rare sinovenous thrombosis. It is defined by the presence of a thrombus in the cerebral veins/dural sinuses or cessation of blood flow with or without parenchymal infarction. It may show a tumor-like, non-specific mass in the posterior fossa. It may regress spontaneously or ischemic brain lesions secondary to thrombosis may cause antenatal or postnatal morbidity and mortality. Fetal MR imaging should be performed in suspected cases. The babies should be monitored closely in terms of serious postnatal problems. 


\section{E1402 - IS FACIAL ASYMMETRY IN THE NEWBORN ALWAYS RELATED TO FACIAL PARALYSIS}

Tuncel D. ${ }^{1}$, Ince Z. ${ }^{1}$, Aygun E. ${ }^{1}$, Aslan M. ${ }^{1}$, Coban A. ${ }^{1}$

Istanbul University, Istanbul Faculty of Medicine, Department of Pediatrics, Division of Neonatology, Istanbul-Turkey ${ }^{1}$

Facial paralysis should be considered in newborns with facial asymmetry. This may be due to birth trauma or developmental causes. In rare cases, facial asymmetry may be caused by isolated congenital agenesis of the mimic muscles of the face. The incidence of congenital facial paralysis is $1.2-2.4$ per 1000 births. A newborn case with agenesis of frontal muscle group is presented as a rare cause of congenital peripheral facial paralysis. A G2P1, 28-yearold mother gave birth by cesarean section to a baby boy with 39 1/7 week of gestational age and $3180 \mathrm{~g}$ birthweight. There were no perinatal complications. The cord blood gases were normal and 1 and 5 minute Apgar scores were 9/9. The parents were non consanguineous and there were no other family members with congenital anomalies. Physical examination revealed that the nasolabial fold was flattened on the right, the right eye was open, and there was drooping of the lower lip which became prominent especially during crying (Picture-1). Other system findings were normal. As there was no history of birth trauma, congenital facial paralysis was considered. Imaging studies for the etiologic investigation and accompanying anomalies including echocardiography, cranial and abdominal ultrasonography, brain and acoustic MRI were normal. The needle electromyography (EMG) showed no bioelectric activity in the right frontal muscle. These findings suggested agenesis of right musculus orbicularis oculi and right musculus frontalis. The baby was discharged at the 15th postnatal day with outpatient follow-up. The same findings persisted in the EMG which was repeated at 3 months-of-age. The outpatient follow up still continues and there is no regression in the physical findings. Physical therapy treatment is in progress. The most frequent cause of facial asymmetry in the newborn is either traumatic or congenital peripheral facial paralysis. Traumatic facial nerve paralysis may be considered in cases with forced prolonged delivery, forceps application, and compression of the maternal sacral bone during face delivery. Unlike congenital facial paralysis, trauma-related nerve injuries resolve within 3-6 months. Congenital facial paralysis may be isolated or associated with genetic syndromes. These include Möbius syndrome, Goldenhar syndrome, CHARGE syndrome, Poland syndrome and hemifacial microsomia. Less frequently agenesis of congenital isolated muscles may mimic nerve paralysis. 


\section{E1408 - EFFICACY OF LEVETIRACETAM AS FIRST LINE THERAPY FOR NEONATAL CLINICAL SEIZURES AND ITS LONG TERM OUTCOME AT 12 MONTHS OF AGE}

Kanmaz S. ${ }^{1}$, Altun Köroğlu Ö. ${ }^{2}$, Terek D. ${ }^{3}$, Serin H. ${ }^{3}$, Şimşek E. ${ }^{3}$, Dokurel İ. ${ }^{3}$, Yılmaz S. ${ }^{3}$, Yalaz M. ${ }^{3}$, Aktan G. ${ }^{3}$, Akisu M. ${ }^{3}$, Kültürsay N. ${ }^{3}$, Gökben S. ${ }^{3}$, Tekgül H. ${ }^{4}$

Ege University Medical Faculty, Pediatrics, Izmir-Turkey ${ }^{1}$

Ege University Medical Faculty, Pediatrics, Izmir-Turkey ${ }^{2}$

Ege University Medical Faculty, Pediatrics, Izmir-Turkey ${ }^{3}$

Ege University Medical Faculty, Pediatrics, Izmir-Turkey ${ }^{4}$

\section{Objective}

To investigate the efficacy of levetiracetam (LEV) as a first-line therapy in neonatal seizures and its long-term neurodevelopmental outcome at 12 months of age

\section{Methods}

Sixty seven neonates, who received intravenous (IV) LEV as a first-line therapy for treating seizures between 2013 and 2017 were retrospectively evaluated. Drug efficacy was assessed using five clinical parameters: (1) seizure cessation time, (2) the use of the antiepileptic drug at discharge, (3) medication cessation time in follow-up, (4) electroencephalography (EEG), (5) the occurrence of post-neonatal epilepsy during follow-up. Neurodevelepmental outcome of the infants were assessed with Ankara Development Screenning Inventory (ADSI) at the 12 months of age.

\section{Results}

Symptomatic seizures were identified in $82 \%$ of the neonates (preterm: $44,65.7 \%$, term: 23 , $34.3 \%)$. The EEG confirmation was obtained in 36 (57.1\%) of the neonates with clinical seizures. On the 7th days of life, LEV was effective as monotherapy in 43 (64\%) neonates, whereas add-on therapy was required in 24 (36\%) neonates. At the 1 year of follow-up, 76\% of infants were achieved drug-free state with LEV monotherapy. The remaining 9 (18\%) infants stayed on LEV monotherapy and 3 (6\%) had LEV+ add-on therapy. A favorable neurodevelopmental outcome was defined in $63.8 \%$ of the infants with ADSI at the end of the 1-year follow-up with LEV monotherapy.

\section{Conclusion}

This retrospective cross-sectional study demonstrated that IV LEV is an effective first-line therapy for treating neonatal clinical seizures and that LEV monotherapy effect was sustained during the long term follow-up. 


\section{E1416 - ROLE OF NEUROPROPHYLAXIS IN PRETERM BIRTH}

Kadivnik M. ${ }^{1}$, Milić Vranješ I. ${ }^{1}$, Košuta Petrović M. ${ }^{2}$, Teodosić A. ${ }^{2}$, Metzger A. ${ }^{3}$, Muller A. ${ }^{1}$, Rubin M. ${ }^{3}$, Kardum D. ${ }^{4}$, Wagner Kostadinović J. ${ }^{5}$

University Hospital, Faculty of Medicine, Department of Obstetrics and Gynecology, Osijek-Croatia $^{1}$

University Hospital, Department of Obstetrics and Gynecology, Osijek Croatia ${ }^{2}$

University Hospital, Department of Obstetrics and Gynecology, Osijek-Croatia ${ }^{3}$

University Hospital, Faculty of Medicine, Department of Pediatrics, Osijek-Croatia ${ }^{4}$

Faculty of Medicine Medical Genetics Laboratory, Department of Medical Biology and Genetics, Osijek-Croatia ${ }^{5}$

\section{Introduction}

The incidence of preterm birth in developed countries is estimated at $7.5 \%$ of all births. Incidence of preterm birth in our Clinic is very similar. One of the most daunting complications of preterm birth is cerebral palsy (CP). It is the most frequent cause of child disability. The prevalence of CP is between 2 and 2.5 per 1000 live born babies. Few studies have proven that $\mathrm{CP}$ was less frequent in preterm babies who had been exposed to magnesium sulphate antenatal.

\section{Objective}

The aim of this review was to evaluate effects of antepartum intravenous magnesium sulphate given to women in danger of preterm birth as the neuroprotection of a new-born. We compared those infants with the ones who didn't receive neuroprotection. Participants and methods: We used data from medical records of patients that had preterm delivery in our Clinic in period from January 1st 2017 till May 1st 2019.We included patients that had delivery between 22nd and 32nd week of gestation. Medical history of total of 130 women were included in our review, of whom 17 in total received neuroprotection with MgSO4. They were administered $4 \mathrm{mg}$ of MgSO4 in bolus during 20 minutes, and therapy was continued with infusion of MgSO $41 \mathrm{mg} / \mathrm{h}$ during 12 hours. We studied medical history of mothers and new-borns. We observed side effects of therapy on mothers. We divided newborns in 2 groups by the fact weather they received MgSO4 neuroprotection before delivery or not. We compared brain ultrasound findings and ophthalmological findings of new-borns divided in those 2 groups. We also compared Apgar scores, length of hospital staying, birth weight of new-borns divided in those 2 groups. For numerological data we used basic mean time interval and distribution. For evaluate differences in described variables for 2 heterogeneous groups we used Mann Whitney u test for nonparametric distribution: for evaluate differences between proportions among independent samples we used $\mathrm{x} 2$ test and Fischer's exact test. A value of $\mathrm{P}<0.05$ was considered significant. Statistic evaluation of data was marked with IBM SPSS statistic program (ver. 15.0, SPSS Inc., Chicago, IL, USA)

\section{Results}

We found no significant difference in brain ultrasound findings and ophthalmological findings between groups of new-borns who received and didn't received neuroprotection (Chi square test, $\mathrm{p}=0.775$ for brain ultrasound findings and $\mathrm{P}=0.809$ for ophthalmological findings). Also we didn't find significant difference in Apgar scores between these 2 groups in 1st and 5th minute ( $\mathrm{P}=0.107$ for AS in 1st minute; $\mathrm{P}=0.869$ for 5 th minute; Mann Whitney test), or for number of days in hospital ( $\mathrm{P}=0.513$, Mann Whitney test). 


\section{Conclusion}

In our review we didn't find significant difference in US and ophthalmological findings between groups of new-borns that received and did not receive neuroprotection. These results might be as such since we had small sample of patients. Bigger and longer studies should be managed, since that kind of studies already confirmed benefits of neuroprotection for preventing of CP in preterm born children. 


\section{Neonatology - Hemodynamic monitoring of the sick neonate}

\section{E1224 - CLINICAL PRESENTATION AND MANAGEMENT OF NEONATAL HYPERTENSION}

Ben Thabet A. ${ }^{1}$, Ben Hamad A. ${ }^{1}$, Feki M. ${ }^{1}$, Charfi M. ${ }^{1}$, Regaieg C. ${ }^{1}$, Hamida N. ${ }^{1}$, Regaieg R. ${ }^{2}$, Bouraoui A. ${ }^{1}$, Gargouri A. ${ }^{2}$

Hedi Chaker Hospital, Neonatology, Sfax-Tunisia ${ }^{1}$

Hedi Chaker Hospitla, Neonatology, Sfax-Tunisia ${ }^{2}$

\section{Objective}

To recall the clinical presentation, causes and therapy approach of neonatal hypertension (HT).

\section{Methods:}

This report is a retrospective study of 12 cases of neonatal hypertension in the neonatology department of Sfax between 2007 and 2017.

\section{Results}

There were 7 boys and 5 girls. The mean term was 34 weeks of gestation (30 to 38 WG). The mean age at diagnosis of hypertension was 7 days (2 to 22 days). A routine monitoring has revealed the HT in 11 cases. Hematuria was seen in one infant. The HT was secondary to the aorta coarctation in one case, compression of the renal pedicle with a cystic lymphangioma in one case, corticotubular necrosis complicating perinatal asphyxia in one case, and umbilical artery catheterization in 9 cases. Arterial thrombosis was recognized in 3 babies.

Nine infants have required continuous intravenous infusions of nicardipine during a period of 5 to 15 days. Oral antihypertensive agent (captopril) was necessary in 4 cases. The hypertension has resolved within neonatal period in 11 cases. The outcome was quiet good for a neonate with corticotubular necrosis.

\section{Conclusion}

Blood pressure in neonates depends on a variety of factors.

Umbilical catheterization is the most common cause of neonatal hypertension. Its use in neonatal intensive care units should be very limited. The long-term prognosis depends on the underlying etiology. 


\section{E1259 - NEONATAL SPONTANEOUS DESCENDING AORTA THROMBOSIS}

Dorobantu R. ${ }^{1}$, Mocuta D. ${ }^{1}$, Filip V. ${ }^{1}$, Aur C. ${ }^{1}$, Dorobantu C. ${ }^{1}$

Faculty of Medicine and Pharmacy, University of Oradea, Oradea-Romania ${ }^{1}$

The well-known conditions that stimulate the excessive thrombin production and the formation of thrombi are: cardiac diseases, polycythemia, kidney diseases, asphyxia, dehydration, septicemia, necrotizing enterocolitis, respiratory distress syndrome (RDS), genetic defects of fibrinolysis (protein $\mathrm{C}$, protein $\mathrm{S}$, antithrombin deficiency), mutations of factors II or V of coagulation.

Thrombophilia can be defined as a predisposition to form clots inappropriately. Thrombotic events are increasingly recognized as a significant cause of mortality and morbidity. The predisposition to form clots can arise from genetic factors, acquired changes in the clotting mechanism, or, more commonly, an interaction between genetic and acquired factors. Spontaneous thrombosis of the aorta in the new-born is known to be a very rare event and to have a high mortality risk.

We will present the case of a male, normal weighted, delivered at term new-born baby with a normal Apgar score. Soon after birth he developed a thrombosis of the thoracic descending aorta with an unknown aetiology, with fulminant evolution to death. In the first 2 days of life, the baby was in the rooming-in department, breastfed. In the 3rd day of life moderate skin pallor appeared, bilateral hydroceles, pelvic moderate oedema and decreased urinary output appeared. Paraclinical tests highlighted metabolic acidosis, normal cells blood count, but an abnormal kidney and liver function. The treatment was total parenteral nutrition with amino acids restriction, Sodium Bicarbonate 4,2\%, Dopamine, diuretics and antibiotherapy. The patient had a diuresis of $1 \mathrm{ml} / \mathrm{kg} /$ day and neurologic impairment with repeated apnoea crisis and hypertonia and he needed mechanical ventilation in SIMV system. In the 4th day appeared profound coma, shock, oedema, and the decreasing of the diuresis to $0,5 \mathrm{ml} / \mathrm{kg} / \mathrm{day}$. The baby died in the 5th day because of liver deficiency, kidney deficiency, severe metabolic acidosis with Base Deficit (-21mEq/l). The following diagnosis was recorded in his medical papers: Multiple organ failure, metabolic acidosis, hyperammonemia, raising the suspicion of a metabolic disease. The anatomopathological diagnosis was: Coarctation of the aorta, thrombosis of the thoracic aorta, liver and kidney deficiency, intraventricular hemorrhage, with the persistence of the suspicion of the metabolic disease. The main discussion was about which was the first event who determined the evolution towards the multiple organ failure: the congenital heart disease, which determined the decrease post stenosis blood flow, or the metabolic disease (from glycogenosis or amino acids group) or thrombophilia?

After clinical aspects, evolution, paraclinical and anatomopathological results we could conclude in this case that the Coarctation of the aorta was the first event and the spontaneous thrombosis at the descending aorta level was secondary to the congenital cardiac disease. 


\section{E1393 - CEREBRAL TISSUE PERFUSION DURING TRANSITIONAL PERIOD IN NEONATES HAVING TENDENCY FOR NEONATAL HYPOGLYCEMIA DEVELOPMENT}

Yasa B. ${ }^{1}$, Gonen I. ${ }^{1}$, Dincer E. ${ }^{1}$, Babayigit A. ${ }^{1}$, Saglam O. ${ }^{1}$, Cetinkaya M. ${ }^{1}$

Kanuni Sultan Suleyman Training and Research Hospital, Neonatology, Istanbul-Turkey ${ }^{1}$

\section{Objective}

Aim of this study is to evaluate the regional cerebral tissue oxygenation and cerebral blood flow within first 24 hours of neonates who have risk for neonatal hypoglycemia (NH) development.

\section{Methods}

We conducted a prospective case control study. A total of 50 neonates were sub-grouped as the study group ( $\mathrm{n}=25)$; neonates who have risk factors for $\mathrm{NH}$ development and the control group $(n=25)$ of healthy term neonates. Continuous regional cerebral tissue oxygenation (rScO2) was measured by near-infrared spectroscopy (NIRS) beginning from the first hour to 24th hours of life. rScO2 and oxygen saturation detected by pulse oximeter were recorded and fractionized tissue oxygen extraction (FTOE) was calculated. Middle cerebral artery (MCA) flow was evaluated by doppler ultrasound through the sphenoidal fontanel twice at the first and 24 hours of life. Angel independent doppler indices such as pulsatility index (PI) and resistive index (RI) were measured.

\section{Results}

The mean gestational age of infants was $36.6 \pm 1.8$ and $37.8 \pm 1.4$ weeks and the mean birthweight of infants was $2748 \pm 785$ and $3035 \pm 614$ grams for study and control groups respectively $(\mathrm{p}>0.05)$. Hemoglobin levels of both groups were similar $(\mathrm{p}>0.05)$. Plasma glucose measurements performed at starvation with 3 hours intervals were similar in both groups ( $\mathrm{p}>0.05)$. Regional cerebral oxygen saturations ( $\mathrm{rScO} 2)$ were continuously measured higher in the study group and significantly higher at 1st, 12th, 21st and 24th hours of life $(p<0.05)$. FTOE values were continuously lower in the study group which were significantly lower at 1st, 3rd, 12th, 21st and 24th hours of life $(\mathrm{p}<0.05)$. The mean MCA Pulsatility Index (PI) values at the first and 24th hours of life were significantly higher in the study group $(\mathrm{p}<0.05)$ and no significant difference in mean MCA Resistive Index (RI) values were detected.

\section{Conclusion}

Increased PI reflects increased vascular resistance and higher $\mathrm{rScO} 2$ values may suggest increased in cerebral perfusion which develops as a compensatory auto-regulatory response mechanism. Significantly lower FTOE values in the study group may dedicate decreased cerebral tissue oxygen extraction that results from impaired cerebral perfusion even in the presence of auto-regulatory mechanisms. We suggest that, even if clinically significant symptoms of neonatal hypoglycemia has not developed, long term neurological outcomes should be followed in infants having risk factors for neonatal hypoglycemia development due to impaired cerebral perfusion. 


\section{E1397 - CHORIOANGIOMA AS A RARE CAUSE OF NON IMMUNE HYDROPS} FETALIS

Aslan M. ${ }^{1}$, Coban A. ${ }^{1}$, Aygun E. ${ }^{1}$, Tuncel D. ${ }^{1}$, Bayraktar Eltutan C. ${ }^{1}$, Sarac Sivrikoz T. ${ }^{2}$, Ince Z. ${ }^{1}$

Istanbul University, Istanbul Faculty of Medicine, Department of Pediatrics, Division of Neonatology, Istanbul-Turkey ${ }^{1}$

Istanbul University, Istanbul Faculty of Medicine, Department of Obstetrics and Gynecology, Division of Perinatology, Istanbul-Turkey ${ }^{2}$

Antenatal ultrasonography (USG) at 20th week of gestation of a G2P1 mother showed a placental mass of size $3 \times 3 \mathrm{~cm}$, suspected to be an organized hematoma or a vascular malformation. After 24 weeks of gestation polyhydramnios developed while the size of the mass gradually increased reaching $11 \times 11 \mathrm{~cm}$ at 30th week. A female baby with hydrops fetalis was delivered by caesarean section due to fetal distress at $31+3 / 7$ weeks of gestation and the placenta was sent for pathological examination. The birthweight of the baby was 2160 g ( $>97 p)$, height $44 \mathrm{~cm}(90-97 p)$, head circumference $32 \mathrm{~cm}(>97 p)$. Apgar scores at 1, 5 and 10 minutes were 5/6/6 respectively, cord blood analysis revealed normal blood gases, total bilirubin level $2.8 \mathrm{mg} / \mathrm{dL}$ and hct $31 \%$. The blood groups of both the mother and the baby were 0 Rh positive, direct Coombs' test was negative. The general condition of the baby was poor; she was hypotonic, pale, with generalized edema, hepatosplenomegaly and ecchymoses on the inguinal region (Figure 1 and 2). The baby was intubated immediately after delivery in the operation room and transferred to the NICU. Pressure support plus volume guarantee ventilation was started and surfactant was administered.

The results of the initial laboratory investigations and imaging studies for the etiology of hydrops fetalis were as follows: serum albumin $1.6 \mathrm{~g} / \mathrm{dL}$, total bilirubin $5.4 \mathrm{mg} / \mathrm{dL}$, calcium $6.4 \mathrm{mg} / \mathrm{dL}$, other electrolytes and urea, creatinine, AST, ALT levels were normal. Echocardiography, transfontanel USG, abdominal USG were normal whereas pleural effusion was detected on chest USG, $8 \mathrm{~mm}$ on the right and $4 \mathrm{~mm}$ on the left side. Expanded newborn screening tests for inherited metabolic diseases, hemoglobin electrophoresis, G6PD levels were normal. Serology for TORCH, syphilis, parvovirus-B19 were negative. Coagulation tests (INR, PT, aPTT) were elevated and the platelet count was $43.000 / \mathrm{mm} 3$. Repeated administration of fresh frozen plasma, albumin, platelet and red blood cell transfusions was needed. On the third postnatal day her general condition improved, edema resolved and she was extubated. Minimal enteral nutrition and TPN were started on the first day of life and full enteral feeding was reached on the 13th postnatal day. The baby needed phototherapy in the the first hours of life, and continued intermittently for a total of 9 days. The result of the pathological examination of the placenta was reported as placental choriangioma which was accepted as the cause of hydrops fetalis (Figure 3 and 4). The patient was discharged on the 24th day of life (Figure 5 and 6).

In conclusion the placental pathologies as a cause of non-immune hydrops fetalis are rare and can be overlooked. Chorioangioma is diagnosed in about $0.5-1 \%$ of placentas. It is frequently associated with fetal and maternal complications such as polyhydramnios, preterm labor, hydrops fetalis, hemolytic anemia, fetal thrombocytopenia, cardiomegaly, intrauterine growth restriction and placental separation. Placental chorioangioma should be considered in the etiology of non-immune hydrops fetalis emphasizing the importance of histopathological examination of the placenta. 


\section{E1467 - NEONATAL PULMONARY HYPERTENSION}

Ben Thabet A. ${ }^{1}$, Bahloul M. ${ }^{1}$, Jdidi J. ${ }^{2}$, Ben Hamad A. ${ }^{1}$, Charfi M. ${ }^{1}$, Regaieg C. ${ }^{1}$, Damak J. ${ }^{2}$, Bouraoui A. ${ }^{1}$, Gargouri A. ${ }^{1}$

Hedi Chaker Hospital, Neonatology, Sfax-Tunisia ${ }^{1}$

Hedi Chaker Hospital, Communautary Department, Sfax-Tunisia ${ }^{2}$

Persistent neonatal pulmonary hypertension (PNPH) complicates the course of approximately $10 \%$ of infants with respiratory failure, and is a source of considerable mortality and morbidity in this population.

\section{Objective}

To describe these neonates and to analysis their outcome

\section{Methods}

This is a retrospective review of neonatal cases of PNPH, within a period of 7 years (2010 to 2017) in the neonatology department of Sfax.

\section{Results}

There were 245 babies. The identified risk factors of PNPH were: male sex (60,4\%), mother diabetes (16.7\%) cesarean delivery (75.9\%), hypotrophy (18.4\%). All patients had respiratory failure. The transitory distress was the most frequent etiology (43.3\%), followed by hyaline membrane disease (18\%), malformations (13.5\%), meconial aspiration syndrome (9.8\%) and infections (9.8\%). Mechanical ventilation was necessary in $79.6 \%$ cases. High frequency oscillation was used in $61.5 \%$ patients. Pulmonary dilatators were used in $39.5 \%$ cases (inhaled nitric oxyde:37.1\%, Sildenafil: 2.4\%). Secondary infections happened in 27.3\% babies. Mortality rate was $28.2 \%$. Neurologic disabilities were noted in $13 \%$ of cases at the age of 12 months. Both mortality and neurologic outcome were statistically associated with severe PNPH, low Apgar score, mechanical ventilation and nitric oxide use.

\section{Conclusion}

The approval of inhaled nitric oxide has dramatically changed treatment for PPHN, although it has not reduced mortality. In addition, developmental deficit remains a serious morbidity after hypoxic consequences of PPHN. 


\title{
Neonatology - Mechanical and noninvasive ventilation
}

\author{
E1276 - EPIDEMIOLOGICAL CLINICAL AND EVOLUTIONARY PROFILE OF \\ HYALINE MEMBRANE DISEASE IN FULL TERM NEW BORN \\ Ben Salem H. ${ }^{1}$, Barbaria W. ${ }^{1}$, Kasdallah N. ${ }^{1}$, Blibech S. ${ }^{1}$, Douagi M. \\ Military Hospital, Nciu, Tunis-Tunisia ${ }^{1}$
}

Hyaline membrane disease (HMD) is a respiratory condition due to functional deficiency in pulmonary surfactant. This pathology is well known in premature neonates but is still unknown or even denied in full term neonates.

Descriptive retrospective study within the NCIU of the Tunisian Military Hospital over a period of 3 years from January 1st, 2016 to December 31st, 2018.

Disease frequency was $12.5 \%$ of the total respiratory distress of full term newborns admitted during the study period. The majority of newborns were "out born". Sex ratio was 1.6. Mean gestational age at birth was $38.12 \pm 0.96$ gestational weeks. The most frequent mode of delivery was caesarian section in $82 \%$ of cases. Mean birth weight was $3306 \pm 520$ g. Mean age of newborns at admission was $12.05 \pm 14.25$ hours. "Out borns" were aged $19.15 \pm 14.43$ hours. Mean age of "in borns" was $1.57 \pm 1.09$. Respiratory distress was immediate for all newborns. Chest-x ray showed alveolar syndrome in $90 \%$ of cases. Association with intrathoracic effusion was noted in $17.6 \%$. All newborns had mechanical ventilation at an average age of $16.88 \pm 14.46$ hours. All newborns were treated with exogenous surfactant at an average age of $21.85 \pm 17.39$ hours. Pulmonary arterial hypertension was found in $58 \%$ of cases. The trend was favorable in $88.2 \%$ of cases. The 4 deaths were attributed to a care-associated infection in one case and persistent pulmonary arterial hypertension in 3 cases. HMD in full term newborn is a real entity. It seems essential to consider this diagnosis in a newborn with respiratory distress in order to avoid any delay in management, source of very serious complications. Elective caesarian section is the main risk factor. We recommend an obstetric-pediatric collaboration to ensure a better prognosis for these newborns. 


\section{Neonatology - Early origins of adult disease}

\section{E1115 - SMALL FOR GESTATIONAL AGE NEWBORNS IN CANTONAL HOSPITAL ZENICA IN THE 6 MONTHS PERIOD A RETROSPECTIVE CASE SERIES STUDY}

Dzidic-Hukeljic L. ${ }^{1}$, Husika M. ${ }^{1}$, Iriškić R. ${ }^{1}$, Beganović S. ${ }^{1}$, Kadribašić J. ${ }^{1}$

Cantonal Hospital Zenica, Department of Gynaecology and Obstetrics-neonatology, Zenica

Bosnia and Herzegovina ${ }^{1}$

\section{Objective}

To identify the prevalence of SGA newborns and most frequently associated risk factors for this condition, evaluate anthropometric characteristics and most commonly present neonatal complications.

\section{Methods}

A retrospective case series study included the history of all SGA newborns in the 6 months period (November 2018-April 2019) in Cantonal Hospital Zenica, Bosnia and Herzegovina. Newborns with birth weight below 10th percentile (adjusted for gestational age and sex) were defined as SGA. The data was collected from the medical records, both obstetric and neonatal.

\section{Results}

In the observed period a total of 1240 live births has been recorded. 43 newborns (3.47\%) were defined SGA. $79.07 \%$ of them were from a singleton and $20.93 \%$ from twin pregnancy. $46.51 \%$ of pregnancies were terminated with a caesarean section, $53.49 \%$ with a vaginal delivery. The median age of mothers at the time of delivery was 28 years. The majority of mothers were nulliparous (65.12\%), 34.88\% multiparous. In $25.58 \%$ pregnancies IUGR has been verified. $16.28 \%$ of mothers had history of PIH, $4.65 \%$ had premature SGA baby born from the previous pregnancy.

Of all SGA newborns $44.19 \%$ were males and 55.81\% females. Regarding the gestational age 86.05\% were born at term, $11.63 \%$ as late-preterm and $2.32 \%$ as preterm (less than $34 \mathrm{wg}$ ). The mean birth weight was $2354.42 \pm 398.49$ grams. Mean ponderal index of all SGA newborns was $2.49 \pm 0.21$. There was no significant difference in comparing PI of newborns with verified IUGR during pregnancy $(2.48 \pm 0.23)$ and newborns without verified IUGR $(2.49 \pm 0.20)$.

In terms of neonatal complications the most frequent was hypothermia; $51.17 \%$ of all SGA newborns (irrespectively of gestational age) were treated in thermoneutral environment for the average duration of 2.47 \pm 1.69 days. Other recorded complications included low blood calcium levels (7.5\%), meconium stained amniotic fluid (7.5\%) with no symptoms of MAS, thrombocytopenia (2.5\%). No birth asphyxia, low blood glucose levels, polycythaemia, PPHN, NEC have been recorded. All newborns survived to discharge. The mean age at discharge was $5.57 \pm 3.79$ days (median 4 days); mean weight at discharge 2353.75 \pm 235.24 grams.

\section{Conclusion}

The prevalence of SGA newborns for the observed period was not high. No serious neonatal complications have been recorded and no case of neonatal death in this group. It is of great importance to identify those high risk newborns and treat them with precaution. Still, all SGA newborns need to be referred to the careful follow-up after the neonatal period, because of remaining higher risk for neurodevelopmental disorders, persistent short stature, obesity, insulin resistance and other metabolic and cardiovascular disorders in the later childhood and adulthood. 


\section{E1291 - 46XX PURE GONADAL DYSGENESIS A DIAGNOSTICAL PROCESS AND EXPECTATIONS OF THE THERAPY}

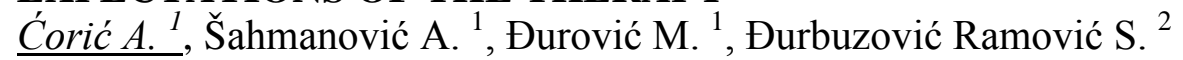

Clinical Center of Montenegro, Obstetrics and Gynecology, Podgorica-Montenegro ${ }^{1}$

General Hospital "danilo I", Obstetrics and Gynecology, Cetinje-Montenegro ${ }^{2}$

\section{Background}

A normal menstrual cycle requires a complex interaction between the ovary/pituitary gland/hypothalamus axis and the genitals. Any alteration such interaction could cause amenorrhoea. There are essentially two types of amenorrhoea: primary amenorrhoea, where the patient has not menstruated yet,and secondary amenorrhoea.Primary amenorrhoea is a present in a patients with normal secondary sexual characteristics, but no menarhe by age 16,or patients who have not had menstrual flow by age 14 and are lacking normal secondary sexual characteristics.

\section{Case presentation}

Woman 23 year old,who is consulted her family doctor worried about her lack of menarche but limited breast development also.Family records not include any serious illness. The patients personal medical record include anomaly of the urinary tract(ren arquatus). A physical exam: height1.75; weight53kg;BMI17.3.She presented armpit and pubic hair. Mammary development was lack. Her external genitals were normal, and her clitoris was of normal size, trough the himen annulare visible is part of the vagina. Blood analyses showed normal haemogram and biochemistry, elevated levels of follicle stimulating hormone(FSH) and luteinizing hormone( $\mathrm{LH})$ and low levels of oestradiol. In September 2018 values were: $\mathrm{FSH}=56.2 \mathrm{mU} / \mathrm{ml} ; \mathrm{LH}=20.8 \mathrm{mU} / \mathrm{ml} ; \mathrm{TSH}=1.94 \mathrm{mU} / \mathrm{ml} ;$ oestradiol $=74 \mathrm{pmol} / \mathrm{L} ;$ prolactine $=138 \mathrm{mI}$ $\mathrm{U} / \mathrm{L}$;progesterone $=1.2 \mathrm{nmol} / \mathrm{L}$; testosterone $=0.92 \mathrm{nmol} / \mathrm{L}$; androstendione $=2.94 \mathrm{nmol} / \mathrm{L}$. A pelvic ultrasound: The uterus measurements 30x19x27mm,endometrial line was not visible. The right side ovary was streak,ultrasonic homogenous, didn't show visible follicles.Her left side ovary presented similar characteristics, measuring $16 \times 19 \mathrm{~mm}$. A vaginoscopy was performed: Vaginal mucosa appears normal, the depth of the vagina was $5-7 \mathrm{~cm}$, it was visible very small cervix with a punctuate ostium. Her peripherical blood karyotype was 46XX.A diagnostic laparoscopy was performed: Uterus with hypoplastic shape and small size ovaries with smooth and nacre coloured surfaces were only discerned.A biopsy of both gonads was performed. The pathological report showed a thinned cortex in both ovaries with a fibrous looking stroma. The primary follicles were absent. Gonadal karyotype was 46XX. Replacement hormonal therapy with oestrogen and progesteron cyclic was applied. Menstrual bleeding has not started after six mounts. Therapy was continued.

\section{Conclusion}

Gonadal dysgenesis is an infrequent cause for primary amenorrhoea. In cases lacking breasts development, a hormonal study should be performed. In the case hypergonadotropic hypogonadismus, karyotype study is recommended.The most common probable cause for primary ovarian failure is autoimmunity, patients also have a higher risk of developing other immunological disorders, immunological screening especially for hypothyroidismus is recommended.A mosaicismus in gonadal karyotype as a frequent cause gonadal dysgenesis, regardless of a normal peripherical karyotype, ovarian biopsy with testing the karyotype of the gonadal tissue is recommended. The lack of female sexual hormones could cause early bone loss and osteoporosis and also represents an important risk factor for neurological, metabolic and cardiovascular health problems, hormonal replacement therapy is recommended.An early diagnosis is extremely important in order to promptly begin treatment 
for the management of symptoms,provide emotional support to the patient and reduce the risks associated with this illness.In case this condition was discovery in early teenage period,it could be expected from this therapy to develop secondary sexual characteristics.However,in your case considering that the patent approached us much later the therapy is applied in order to prevent low oestrogen condition diseases. 